\title{
Trajektorienplanung zur Kollisionsvermeidung im Straßenverkehr
}

\author{
DISSERTATION \\ eingereicht zur Erlangung \\ des akademischen Grades
}

Doktor Ingenieur

an der

Fakultät für Elektrotechnik und Informationstechnik

Technische Universität Dortmund

von

Dipl.-Ing. Martin Keller

aus Castrop-Rauxel

Tag der Einreichung: 07. März 2017

Erster Gutachter: Univ.-Prof. Dr.-Ing. Prof. h.c. Dr. h.c. Torsten Bertram

Zweiter Gutachter: Univ.-Prof. Dr.-Ing. Hans-Christian Reuss

Tag des Kolloquiums: 18. Juli 2017 


\section{Vorwort}

Die vorliegende Arbeit entstand während meiner Tätigkeit als wissenschaftlicher Mitarbeiter am Lehrstuhl für Regelungssystemtechnik der Fakultät für Elektrotechnik und Informationstechnik der Technischen Universität Dortmund und resultiert aus einem kooperativen Forschungsprojekt mit dem Unternehmen TRW Automotive GmbH.

Mein ganz besonderer Dank gilt Herrn Professor Dr.-Ing. Prof. h.c. Dr. h.c. Torsten Bertram für die Möglichkeit zur freien Gestaltung meiner wissenschaftlichen Arbeit und die ausgezeichnete Betreuung, welche diese Arbeit überhaupt erst ermöglichte.

Ebenfalls möchte ich Herrn Professor Dr.-Ing. Hans-Christian Reuss für das der Arbeit entgegengebrachte Interesse und die Übernahme des Mitberichts danken.

Ich danke Herrn Professor Dr.-Ing. Stephan Frei als drittem Prüfer und Herrn Professor Dr.-Ing. Andreas Neyer für die Übernahme des Vorsitzes der Prüfungskommission.

Bei allen Mitarbeitern des Lehrstuhls für Regelungssystemtechnik bedanke ich mich für die angenehme Arbeitsatmosphäre. Herrn apl. Professor Dr. rer. nat. Frank Hoffmann danke ich für zahlreiche Anregungen und Ratschläge. Dr.-Ing. Jörn Malzahn gilt mein Dank für hilfreiche Diskussionen über regelungstechnische Fragestellungen. Artemi Makarow danke ich für seine umfassende und unermüdliche Unterstützung in jeder Hinsicht. Christoph Rösmann gilt mein Dank für die Bereitstellung des MPC Frameworks, Christian Götte und Andreas Homann für den regen Austausch im Bereich der Fahrzeugsystemtechnik. Meinen langjährigen Bürokollegen Dr.-Ing. Javier Antonio Oliva Alonso, Malte Oeljeklaus und Christian Wissing danke ich für den kollegialen Umgang. Für die stets freundliche Unterstützung im technischen und administrativen Bereich bedanke ich mich bei Gabriele Rebbe, Mareike Leber, Jürgen Limmhoff und Rainer Müller-Burtscheid.

Einige Ideen der vorliegenden Arbeit entstanden auch im Rahmen von studentischen Arbeiten. Den beteiligten Studierenden möchte ich ebenfalls danken.

Allen beteiligten Mitarbeitern der TRW Automotive GmbH danke ich für die gute Zusammenarbeit. Dr.-Ing. Alois Seewald gilt mein Dank für die Förderung des Kooperationsprojektes. Bei Dr.-Ing. Carsten Haß bedanke ich mich für die Initiierung des Kooperationsprojektes sowie für die sehr gute und persönliche Betreuung. Markus 
Buß möchte ich für die technische Unterstützung und für viele unterhaltsame Stunden und Tage auf der Teststrecke danken.

Mein Dank gilt auch meiner Familie und meinen Freunden, welche ein Umfeld geschaffen haben, welches zum Gelingen der Arbeit beigetragen hat. Ganz besonders danke ich meinen Eltern Heidrun Ingrid und Rainer Jürgen Keller für die Unterstützung auf meinem Lebensweg.

Meiner Lebensgefährtin Sandy Hentschel danke ich ganz herzlich für die jahrelange Unterstützung und das entgegengebrachte Verständnis. 


\section{Inhaltsverzeichnis}

Nomenklatur

1. Einleitung 1

1.1. Motivation . . . . . . . . . . . . . . . . . . . . 1

1.2. Grundaufgaben eines Kollisionsvermeidungssystems . . . . . . . . . . . . 3

1.3. Stand der Forschung und Technik . . . . . . . . . . . . . . . . . . . . . . 4

1.4. Inhalt und Ziel der Arbeit . . . . . . . . . . . . . . . . . . . . . . . . 12

1.5. Gliederung . . . . . . . . . . . . . . . . . . 13

$\begin{array}{ll}\text { 2. Entwicklungsumgebungen } & 14\end{array}$

2.1. Durchgängige Entwicklungsmethode . . . . . . . . . . . . . . . . . 14

2.2. Fahrsimulator . . . . . . . . . . . . . . . . . . 16

2.3. Anwendung des Fahrsimulators in der Probandenstudie . . . . . . . . . 18

2.4. Prototypisches Versuchsfahrzeug . . . . . . . . . . . . . . 21

3. Prädiktionsmodelle 25

3.1. Massenpunktmodell . . . . . . . . . . . . . . . . . . . 25

3.2. Fahrdynamikmodelle . . . . . . . . . . . . . . . . . . . . 26

3.3. Modellierung der Aktuatorregelkreise . . . . . . . . . . . . . . . . . . . 31

3.4. Auswahl des vollständigen Prädiktionsmodells . . . . . . . . . . . . 32

\begin{tabular}{lll}
\hline 4. Bahnfolgeverfahren & 35
\end{tabular}

4.1. Beschreibung von Ausweichbahnen durch Funktionen . . . . . . . . . 36

4.2. Kritikalitätsmaß und Eingriffsentscheidung . . . . . . . . . . . . . . . 37

4.3. Fahrzeugquerregelung . . . . . . . . . . . . . . . . . . . 39

4.4. Fahrzeuglängsregelung . . . . . . . . . . . . . . . . . . . . 43

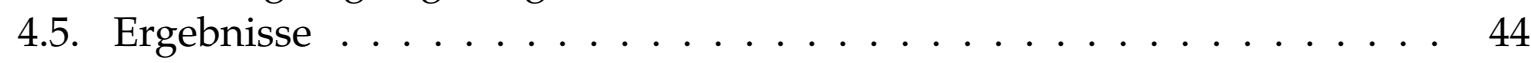

4.6. Verifikation im Versuchsfahrzeug. . . . . . . . . . . . . . . . . . . . 49

5. Online-Trajektorienoptimierungsverfahren 51

5.1. Beschreibung der Fahrzeugbewegung mit Timed Elastic Bands . . . . . 52

5.2. Entwurf des Gütemaßes zur Kollisionsvermeidung . . . . . . . . . . . . 54

5.2.1. Anforderungen an das Gütemaß . . . . . . . . . . . . . . . . . . 54

5.2.2. Aufbau des Gütemaßes . . . . . . . . . . . . . . . . . . 55

5.2.3. Zielkonflikte zwischen Gütemaßanteilen . . . . . . . . . . . . . 57 
5.2.4. Kritikalitätsmaß und Eingriffsentscheidung . . . . . . . . . . . . . . 58

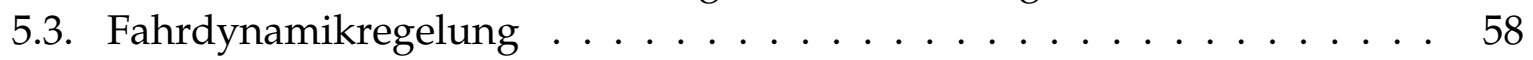

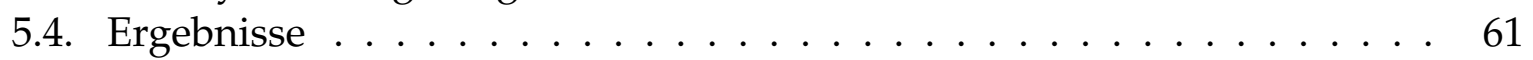

6. Modellprädiktives Planungs- und Regelungsverfahren 65

6.1. Formulierung des Optimalsteuerungsproblems . . . . . . . . . . . . 68

6.2. Entwurf des Gütemaßes zur Kollisionsvermeidung . . . . . . . . . . . . . . 69

6.2.1. Aufbau des Gütemaßes . . . . . . . . . . . . . . . . . . . 69

6.2.2. Zielkonflikte zwischen Gütemaßanteilen . . . . . . . . . . . . . . 70

6.2.3. Wahl der Gewichtungsfaktoren . . . . . . . . . . . . . . . . . . . 71

6.2.4. Kritikalitätsmaß und Eingriffsentscheidung . . . . . . . . . . . 72

6.3. Ergebnisse . . . . . . . . . . . . . . . . . . . 72

7. Modellprädiktives Trajektorienscharverfahren 76

7.1. Trajektorienschar und suboptimale Lösung des Optimalsteuerungspro-

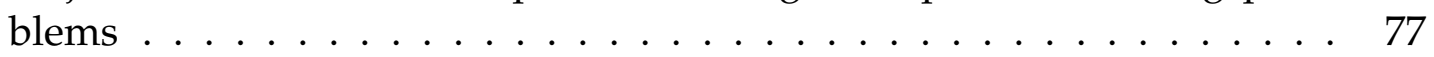

7.2. Adaptive Stellgrößendiskretisierung . . . . . . . . . . . . . . . . 78

7.3. Entwurf des Gütemaßes zur Kollisionsvermeidung . . . . . . . . . . . . 79

7.3.1. Aufbau des Gütemaßes . . . . . . . . . . . . . . . . . . . . 79

7.3.2. Wahl der Gewichtungsfaktoren . . . . . . . . . . . . . . . . . . . 80

7.3.3. Kritikalitätsmaß und Eingriffsentscheidung . . . . . . . . . . . . 81

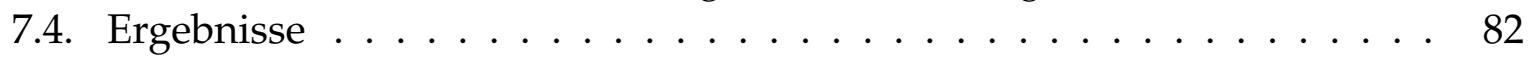

\begin{tabular}{lll}
\hline 8. Analyse, Vergleich und Bewertung der Verfahren & $\mathbf{8 7}$
\end{tabular}

8.1. Auswahl von Vergleichsmethoden . . . . . . . . . . . . . . . . . . . 87

8.2. Vergleich in einer Beispielsituation . . . . . . . . . . . . . . . . . . . . . . . . . 88

8.3. Vergleich mit der Nutzwertanalyse . . . . . . . . . . . . . . . . . . . . . . 90

8.4. Modifikationen und Kombinationen der Verfahren . . . . . . . . . . . . 95

8.5. Fazit . . . . . . . . . . . . . . . . . . . 97

$\begin{array}{ll}\text { 9. Zusammenfassung und Ausblick } & 99\end{array}$

\begin{tabular}{ll}
\hline A. Anhang & 101
\end{tabular}

A.1. Definitionen . . . . . . . . . . . . . . . . . . . . . . . . . . . . . . . . . . . 101

A.1.1. Begriffe des Straßenbaus . . . . . . . . . . . . . . . . . . . 101

A.1.2. Begriffe der Fahrzeugführung . . . . . . . . . . . . . . . . . . . . 102

A.1.3. Zur Kritikalität von Verkehrssituationen . . . . . . . . . . . . . . 103

A.1.4. Weitere Definitionen . . . . . . . . . . . . . . . . . 106

A.2. Modellprädiktive Trajektorienscharregelung . . . . . . . . . . . . . 107

\begin{tabular}{ll}
\hline Literatur & 113
\end{tabular} 


\begin{tabular}{|c|c|}
\hline \multicolumn{2}{|c|}{ Abkürzungen und Akronyme } \\
\hline ABS & Anti-lock Braking System \\
\hline $\mathrm{ACC}$ & Adaptive Cruise Control \\
\hline $\mathrm{ACR}$ & Active Control Retractor \\
\hline AEB & Autonomous Emergency Braking \\
\hline ASR & Antriebsschlupfregelung \\
\hline AWP & Anfangswertproblem \\
\hline CAN & Controller Area Network \\
\hline ESA & Emergency Steering Assist \\
\hline ESC & Electronic Stability Control \\
\hline ESM & Einspurmodell \\
\hline HMI & Human Machine Interface \\
\hline KVT & Kollisionsvermeidungstrajektorie \\
\hline LGA & Lane Guidance Assist \\
\hline LKA & Lane Keeping Assist \\
\hline LKW & Lastkraftwagen \\
\hline LRW & Lenkradwinkel \\
\hline LWR & Lenkwinkelregler \\
\hline MPC & Model Predictive Control \\
\hline MPPC & Model Predictive Planning and Control \\
\hline MPTSA & Model Predictive Trajectory Set Approach \\
\hline MPTSC & Model Predictive Trajectory Set Control \\
\hline MSR & Motorschleppmomentenregelung \\
\hline NRMSE & Normalised Root Mean Squared Error \\
\hline OTO & Online-Trajectory Optimization \\
\hline PFA & Path Following Approach \\
\hline PKW & Personenkraftwagen \\
\hline $\mathrm{RCP}$ & Rapid Control Prototyping \\
\hline SP & Schwerpunkt \\
\hline TEB & Timed Elastic Band \\
\hline TTC & Time To Collision \\
\hline bzw. & beziehungsweise \\
\hline usw. & und so weiter \\
\hline z.B. & zum Beispiel \\
\hline
\end{tabular}




\section{Griechische Symbole}

$\Delta_{\max }$

$\Delta \Psi$

$\Delta T$

$\Delta t$

$\Delta X$

$\Delta Y$

$\Gamma$

$\Gamma_{1}$

$\Gamma_{\mathrm{r}}$

$\Gamma_{\mathrm{pf}, k}$

$\Phi$

$\Psi$

$\dot{\Psi}_{\lim }$

$\dot{\Psi}_{\text {lim,krit }}$

$\Psi_{\text {lim,krit }}$

$\ddot{\Psi}_{\text {lim,stat }}$

$\dot{\Psi}_{\text {lim,stat }}$

$\dot{\Psi}_{\text {mess }}$

$\dot{\Psi}_{\text {sim }}$

$\dot{\Psi}_{\text {soll }}$

$\dot{\Psi}_{\text {soll,ff }}$

$\dot{\Psi}_{\text {stat }}$

$\alpha$

$\alpha_{\mathrm{h}}$

$\alpha_{\mathrm{v}}$

$\alpha_{\mathrm{v}, \max }$

$\alpha_{\mathrm{v}, \min }$

$\beta$

$\beta_{\text {lim,krit }}$

$\beta_{\text {lim,stat }}$

$\dot{\beta}_{\text {lim,stat }}$

$\beta_{\text {stat }}$

$\delta$

$\delta_{\mathrm{aD}}$

$\delta_{\mathrm{ID}}$

$\delta_{\max }$

$\dot{\delta}_{\max }$

$\delta_{\text {max,dis }}$

$\delta_{\text {min }}$
$\dot{\delta}_{\text {min }}$ maximale Abweichung der Istbahn von der Sollbahn

relativer Gierwinkel zur Fahrbahn

Zeitdifferenz

Schrittweite des Euler-Vorwärts-Integrationsverfahrens

Abstand des Schwerpunktes des Hindernisses zur Wendestelle der Sigmoidfunktion des Umgebungsmodells

Entfernung des Fahrzeugschwerpunktes zur Spurmitte

Kostenterm

linker Teil des Umgebungsmodell

rechter Teil des Umgebungsmodell

Umgebungsmodell des MPTSA

relativer Gierwinkel zwischen Ego- und Hindernisfahrzeug

Gierwinkel

unterer Grenzwert der Gierrate

Grenzwert der Gierrate für die Bewertung der Kritikalität

Grenzwert des Gierwinkels für die Bewertung der Kritikalität

Grenzwert der Gierbeschleunigung für einen stationären $\mathrm{Zu}$ stand

Grenzwert der Gierrate für die stationäre Kreisfahrt

gemessene Gierrate

simulierte Gierrate

Sollgierrate

Vorsteueranteil des Positionsreglers

Kreisfahrtwerte der Gierrate

Schräglaufwinkel

Schräglaufwinkel hinten

Schräglaufwinkel vorne

maximaler Schräglaufwinkel (Untersteuergrenze)

minimaler Schräglaufwinkel (Untersteuergrenze)

Schwimmwinkel

Grenzwert des Schwimmwinkels für die Bewertung der Kritikalität

Grenzwert des Schwimmwinkels für die stationäre Kreisfahrt

Grenzwert der Schwimmwinkeländerung für einen stationären

Zustand

Kreisfahrtwerte des Schwimmwinkels

Lenkradwinkel

adaptiv diskretisierter Lenkradwinkel

linear (äquidistant) diskretisierter Lenkradwinkel

maximaler Lenkradwinkel (Untersteuergrenze)

obere Grenze des Betrages der Lenkrate

maximaler Solllenkradwinkel für die diskrete Menge an mögli-

chen Lenkradwinkeln

minimaler Lenkradwinkel (Untersteuergrenze)

untere Grenze des Betrages der Lenkrate 


$\begin{array}{ll}\delta_{\mathrm{SH}} & \text { Lenkwinkelsollwert des Spurhaltereglers } \\ \delta_{\mathrm{soll}} & \text { Solllenkradwinkel } \\ \delta_{\mathrm{SW}} & \text { Lenkwinkelsollwert des Gierratenreglers für Spurwechsel } \\ \delta_{\mathrm{V}} & \text { Radlenkwinkel } \\ \epsilon & \text { Abstandsparameter der Soft-Constraint Funktion } \\ \gamma & \text { Gewichtung } \\ \gamma_{\mathrm{a}} & \text { Gewichtung der Beschleunigung } \\ \gamma_{\mathrm{a}, \mathrm{SC}} & \text { Gewichtung der Soft-Constraints der Beschleunigung } \\ \gamma_{\mathrm{env}} & \text { Gewichtung des Umgebungsmodells } \\ \gamma_{\mathrm{j}} & \text { Gewichtung des Rucks } \\ \gamma_{\mathrm{j}, \mathrm{SC}} & \text { Gewichtung der Soft-Constraints des Rucks } \\ \gamma_{\lambda} & \text { Gewichtung des Kurswinkels } \\ \gamma_{\Psi} & \text { Gewichtung des Gierwinkels } \\ \gamma_{\mathrm{s}} & \text { Gewichtung des Sollschlupfes } \\ \gamma_{\mathrm{sys}} & \text { Gewichtung der Gleichheitsnebenbedingung des MPPC (Einhal- } \\ & \text { tung der Systemdynamik) } \\ \gamma_{\mathrm{u}} & \text { Gewichtung der Ungleichheitsnebenbedingung des MPPC (Stell- } \\ & \text { größenbeschränkung) } \\ \gamma_{\mathrm{X}} & \text { Gewichtung der Längsabweichung von der Solltrajektorie } \\ \gamma_{\mathrm{Y}} & \text { Gewichtung der Querabweichung von der Solltrajektorie } \\ \lambda & \text { Kurswinkel } \\ \omega & \text { Raddrehzahl } \\ \tau & \text { Menge der Zeitdifferenzen } \\ \Gamma_{\mathrm{env}} & \text { Umgebungsmodell }\end{array}$

\section{Lateinische Symbole}

$\begin{array}{ll}A & \text { Menge der diskreten Solllenkradwinkel } \\ B & \text { Menge der diskreten Sollschlupfwerte } \\ B_{\mathrm{LWR}} & \text { Funktion zur Begrenzung der Lenkrate } \\ B_{\mathrm{y}}, B_{\mathrm{x}} & \text { Parameter der Reifenkennlinien } \\ C_{\mathrm{y}}, C_{\mathrm{x}}, C_{\mathrm{a}}, C_{\mathrm{s}} & \text { Parameter der Reifenkennlinien } \\ D & \text { Differentialregleranteil } \\ D_{\mathrm{y}}, D_{\mathrm{x}}, D_{\mathrm{a}}, D_{\mathrm{s}} & \text { Parameter der Reifenkennlinien } \\ E_{\mathrm{g}} & \text { exponentieller Gewichtungsfaktor } \\ E_{\mathrm{y}}, E_{\mathrm{x}} & \text { Parameter der Reifenkennlinien } \\ F_{\mathrm{max}} & \text { Maximalkraft } \\ F_{\mathrm{tot}} & \text { Gesamtkraft } \\ F_{\mathrm{x}} & \text { Längskraft (allgemein) } \\ F_{\mathrm{x} 0} & \text { Längskraft ohne Querschlupf } \\ F_{\mathrm{xh}} & \text { Längskraft Hinterrad } \\ F_{\mathrm{xv}} & \text { Längskraft Vorderrad } \\ F_{\mathrm{xvr}} & \text { Längskraft Vorderrad in Radkoordinaten } \\ F_{\mathrm{y}} & \text { Querkraft (allgemein) } \\ F_{\mathrm{y} 0} & \text { Querkraft ohne Längsschlupf } \\ F_{\mathrm{yh}} & \text { Querkraft Hinterrad } \\ F_{\mathrm{yv}} & \text { Querkraft Vorderrad }\end{array}$




\begin{tabular}{|c|c|}
\hline$F_{\text {yvr }}$ & Querkraft Vorderrad in Radkoordinaten \\
\hline$G_{\text {LS }}$ & Übertragungsfunktion des Lenksystems \\
\hline$G_{x}$ & Gewichtungsfaktor der Längskraft (allgemein) \\
\hline$G_{\mathrm{xh}}$ & Gewichtungsfaktor der Längskraft Hinterachse \\
\hline$G_{\mathrm{xvr}}$ & Gewichtungsfaktor der Längskraft Vorderachse \\
\hline$G_{y}$ & Gewichtungsfaktor der Querkraft (allgemein) \\
\hline$G_{\text {yh }}$ & Gewichtungsfaktor der Querkraft Hinterachse \\
\hline$G_{y v r}$ & Gewichtungsfaktor der Querkraft Vorderachse \\
\hline I & Integralregleranteil \\
\hline$J_{\mathrm{a}, \mathrm{y}}$ & $\begin{array}{l}\text { Gütemaß zur Bewertung der Abweichung zwischen der gemes- } \\
\text { senen und der simulierten Querbeschleunigung }\end{array}$ \\
\hline$J_{\mathrm{ESM}}$ & Gütemaß zur Identifikation des Einspurmodells \\
\hline$J_{\text {LA }}$ & Trägheit des Lenkaktuators \\
\hline$J_{\text {LS }}$ & Gütemaß des Riccati-Reglers des Lenksystems \\
\hline$J_{\text {Lag }}$ & Langrangesches Gütemaß \\
\hline$J_{\text {Lag,MPTSC }}$ & Lagrangesches Gütemaß des MPTSC \\
\hline$J_{\text {Lag,Ric }}$ & Lagrangesches Gütemaß des Riccati-Reglers \\
\hline$J_{\mathrm{MPPC}}$ & Gütemaß des MPPC \\
\hline$J_{\text {MPTSA }}$ & Gütemaß des MPTSA \\
\hline$J_{\text {MPTSC }}$ & Gütemaß des MPTSC \\
\hline$J_{\mathrm{MR}}$ & Gütemaß zur Optimierung des MPTSC \\
\hline$J_{\dot{\Psi}}$ & $\begin{array}{l}\text { Gütemaß zur Bewertung der Abweichung zwischen der gemes- } \\
\text { senen und der simulierten Gierrate }\end{array}$ \\
\hline$J_{\text {Ric }}$ & $\begin{array}{l}\text { Gütemaß zur Optmierung der Gewichtungsmatrizen des Riccati- } \\
\text { Reglers }\end{array}$ \\
\hline$J_{\mathrm{TEB}}$ & Gütemaß des TEB \\
\hline$J_{\mathrm{z}}$ & Gierträgheitsmoment des Fahrzeugs \\
\hline$K_{\text {ges }}$ & Gesamtkritikalität einer Verkehrssituation \\
\hline$K I B$ & $\begin{array}{l}\text { Kritikalitätsindikator bezüglich des Nutzungsgrades der Bewe- } \\
\text { gungspotentiale }\end{array}$ \\
\hline KIU & $\begin{array}{l}\text { Kritikalitätsindikator bezüglich der Unsicherheit über die Bewe- } \\
\text { gung der Verkehrsteilnehmer }\end{array}$ \\
\hline$K_{\text {pot }}$ & $\begin{array}{l}\text { Kritikalität bezüglich des Nutzungsgrades der Bewegungspoten- } \\
\text { tiale }\end{array}$ \\
\hline$K_{\text {uns }}$ & $\begin{array}{l}\text { Kritikalität bezüglich der Unsicherheit über die Bewegung der } \\
\text { Verkehrsteilnehmer }\end{array}$ \\
\hline$M_{\mathrm{BS}}$ & Führungsfilter des Beispielsystems \\
\hline$P$ & Proportionalregleranteil \\
\hline$P_{k}$ & Punkte des TEB \\
\hline$Q_{\text {dyn }}$ & dynamische Größe \\
\hline$Q_{\text {dyn,max }}$ & Maximalwert einer dynamischen Größe \\
\hline$Q_{\mathrm{TEB}}$ & Menge der Punkte des Timed Elastic Band \\
\hline$S T$ & Strafterm zur Bestrafung von Kollisionen \\
\hline$T_{\mathrm{a}}$ & Unterstützendes Lenkmoment \\
\hline$T_{\mathrm{BS}}$ & Zeitkonstante des Beispielsystems \\
\hline$T_{\mathrm{d}}$ & Lenkmoment (Drehmomentregler) \\
\hline
\end{tabular}




\begin{tabular}{|c|c|}
\hline TEB & Timed Elastic Band \\
\hline$T_{\mathrm{F}}$ & Lenkmoment des Fahrers \\
\hline$T_{\text {Kol }}$ & Horizont zur Überwachung von Kollisionen \\
\hline$T_{\text {LWR }}$ & Zeitkonstante des Lenkradwinkelregelkreises \\
\hline$T_{\mathrm{m}}$ & Lenkmoment (Microcontroller) \\
\hline$T_{\mathrm{O}}$ & Lenkmomentenüberlagerung (Torque Overlay) \\
\hline$T_{\mathrm{pr}}$ & Prädiktionszeit \\
\hline$T_{\mathrm{r}}$ & Lenkmoment (RCP System) \\
\hline$T_{\text {rück }}$ & Rückstellmoment der Vorderreifen \\
\hline$T_{\mathrm{S}}$ & Zeitkonstante des Schlupfregelkreises \\
\hline$T_{\text {sen }}$ & am Torsionsstab gemessenes Drehmoment \\
\hline$T_{\sum_{1}}$ & Summenzeitkonstante \\
\hline$T_{\mathrm{TC}}$ & Zeitkonstante des Drehmomentregelkreises \\
\hline$U W E$ & Überschwingweite \\
\hline$X$ & erdfeste Koordinate \\
\hline$X_{\Delta, \max }$ & $\begin{array}{l}\text { Stelle in erdfesten Koordinaten, an der die maximale Abwei- } \\
\text { chung der Istbahn von der Sollbahn auftritt }\end{array}$ \\
\hline$X_{\mathrm{e}}$ & Endposition des Spurwechselpolynoms \\
\hline$X_{\text {Obs }}$ & Schwerpunktkoordinate des Hindernisses \\
\hline$X_{\mathrm{Oe}}$ & Position der hinteren Kante des Hindernisses \\
\hline Y & erdfeste Koordinate \\
\hline$Y_{0}$ & Startwert der Sigmoidfunktion des Umgebungsmodells \\
\hline$Y_{\mathrm{e}}$ & Endposition des Spurwechselpolynoms \\
\hline$Y_{\text {end }}$ & Endwert der Sigmoidfunktion des Umgebungsmodells \\
\hline$Y_{\max }$ & oberer Grenzwert des Umgebungsmodells \\
\hline$Y_{\min }$ & unterer Grenzwert des Umgebungsmodells \\
\hline$Y_{\text {Obs }}$ & Schwerpunktkoordinate des Hindernisses \\
\hline$Y_{\text {Oe }}$ & Position der hinteren Kante des Hindernisses \\
\hline$Y_{\text {pol }}$ & Spurwechselpolynom \\
\hline$Y_{\text {safe }}$ & Position mit Sicherheitsabstand zum Hindernis \\
\hline$Y_{\text {soll }}$ & Sollbahn \\
\hline$a$ & Beschleunigung \\
\hline$a_{0 . . .5}$ & Polynomkoeffizienten \\
\hline$a D_{0 \ldots 4}$ & Koeffizienten des Polynoms für die adaptive Diskretisierung \\
\hline$a_{\max }$ & maximale Gesamtbeschleunigung \\
\hline$a_{\mathrm{n}}$ & Normalbeschleunigung \\
\hline$a_{\mathrm{t}}$ & Tangentialbeschleunigung \\
\hline$a_{\text {tot }}$ & Gesamtbeschleunigung \\
\hline$a_{\mathrm{x}}$ & Längsbeschleunigung \\
\hline$a_{\mathrm{x}, \text { soll }}$ & Solllängsbeschleunigung \\
\hline$a_{\mathrm{y}}$ & Querbeschleunigung \\
\hline$a_{\mathrm{y}, \mathrm{lim}}$ & unterer Grenzwert der Querbeschleunigung \\
\hline$a_{\mathrm{y}, \mathrm{mess}}$ & gemessene Querbeschleunigung \\
\hline$a_{\mathrm{y}, \mathrm{sim}}$ & simulierte Querbeschleunigung \\
\hline$b_{\mathrm{LA}}$ & Viskose Reibung des Lenkaktuators \\
\hline$b_{\mathrm{Obs}}$ & Breite des Hindernisses \\
\hline
\end{tabular}




\begin{tabular}{|c|c|}
\hline$c_{\mathrm{i}}$ & Polynomkoeffizienten \\
\hline$d_{1}, d_{2}$ & Abstandsparameter des Umgebungsmodells des MPTSA \\
\hline$d_{\mathrm{BS}}$ & Dämpfungskonstante des Beispielsystems \\
\hline$d_{\text {Lag }}$ & relative Änderung des Lagrangeschen Gütemaßes \\
\hline$d_{\text {Limit }}$ & Grenzwert des Umgebungsmodells des MPTSA \\
\hline$d_{\mathrm{LWR}}$ & Dämpfungskonstante des Lenkradwinkelregelkreises \\
\hline$d_{\min , \mathrm{x}}$ & $\begin{array}{l}\text { Abstand des Hindernisses zur Wendestelle der Sigmoidfunktion } \\
\text { des Umgebungsmodells }\end{array}$ \\
\hline$d_{\min , Y}$ & $\begin{array}{l}\text { Abstand des Hindernisses zum oberen Grenzwert der Sigmoid- } \\
\text { funktion des Umgebungsmodells }\end{array}$ \\
\hline$d_{\mathrm{Obs}}$ & $\begin{array}{l}\text { kleinster Abstand der vier Ecken des Ego-Fahrzeugs zum Schwer- } \\
\text { punkt des Hindernisses }\end{array}$ \\
\hline fit & Fit-Wert \\
\hline$f_{0 \mathrm{bs}}$ & Funktion des Umfeldmodells \\
\hline$f_{\mathrm{p}}$ & Formungsparameter des Umgebungsmodells des MPTSA \\
\hline$f_{\mathrm{SC}}$ & Soft-Constraint Funktion \\
\hline g & Erdbeschleunigung \\
\hline$g_{\mathrm{u}}$ & Gewichtungsfunktion einer Stellgröße \\
\hline$g_{\mathrm{y}}$ & Gewichtungsfunktion einer Ausgangsgröße \\
\hline$h$ & Gleichheitsnebenbedingung des MPPC \\
\hline$i, j, k$ & Laufindex \\
\hline$i_{\mathrm{s}}$ & Lenkübersetzung \\
\hline$j_{\mathrm{n}}$ & Normalruck \\
\hline$j_{\mathrm{t}}$ & Tangentialruck \\
\hline$k_{\mathrm{LS}}$ & Verstärkungsfaktor des Lenksystems \\
\hline$k_{\mathrm{LWR}}$ & Verstärkungsfaktor des Lenkradwinkelregelkreises \\
\hline$k_{\mathrm{s}}$ & Verstärkungsfaktor des Schlupfregelkreises \\
\hline$l_{\mathrm{h}}$ & Abstand der Hinterachse vom Fahrzeugschwerpunkt \\
\hline$l_{\text {Obs }}$ & Länge des Hindernisses \\
\hline$l_{\mathrm{v}}$ & Abstand der Vorderachse vom Fahrzeugschwerpunkt \\
\hline$m$ & Fahrzeugmasse \\
\hline$n_{\mathrm{c}}$ & Kontrollhorizont \\
\hline$n_{\gamma}$ & Anzahl der Kostenterme des TEB \\
\hline$n_{\mathrm{p}}$ & Prädiktionshorizont \\
\hline$r_{\mathrm{Bx} 1}, r_{\mathrm{Bx} 2}, r_{\mathrm{By} 1}, r_{\mathrm{By} 2}$ & Parameter der Reifenkennlinien \\
\hline$r_{\text {dyn }}$ & Dynamischer Radhalbmesser \\
\hline$s$ & Laplace Variable \\
\hline$s_{\mathrm{hl}}$ & Schlupf des hinteren linken Rades \\
\hline$s_{\mathrm{hr}}$ & Schlupf des hinteren rechten Rades \\
\hline$s_{1}$ & Schlupf \\
\hline$s_{1, \min }$ & unterer Sollschlupfgrenzwert \\
\hline$s_{\text {soll }}$ & Sollschlupf \\
\hline$s_{\mathrm{vl}}$ & Schlupf des vorderen linken Rades \\
\hline$s_{\mathrm{vr}}$ & Schlupf des vorderen rechten Rades \\
\hline$t$ & Zeit \\
\hline$t_{\text {la }}$ & Vorausschauzeit \\
\hline
\end{tabular}




$\begin{array}{ll}u_{\mathrm{BS}, \text { max }} & \text { obere Stellgrößenbeschränkung des Beispielsystems } \\ u_{\mathrm{BS}, \text { min }} & \text { untere Stellgrößenbeschränkung des Beispielsystems } \\ v & \text { Fahrzeuggeschwindigkeit } \\ v_{\text {init }} & \text { initiale Geschwindigkeit, mit der ein Notmanöver begonnen } \\ & \text { wird } \\ v_{\mathrm{x}, \text { rad }} & \text { Längsgeschwindigkeit in Radkoordinaten } \\ v_{\mathrm{y}, \text { rad }} & \text { Quergeschwindigkeit in Radkoordinaten } \\ y_{\mathrm{BS}} & \text { Ausganggröße des Beispielsystems } \\ y_{\mathrm{MPTSC}} & \text { Ausgangsgröße des mit einem MPTSC geregelten Systems } \\ y_{\text {Ref }} & \text { Referenzgröße } \\ y_{\text {Ric }} & \text { Ausgangsgröße des mit einem Riccati-Regler geregelten Systems } \\ z & \text { Variable der Soft-Constraint Funktion } \\ z_{\mathrm{m}} & \text { Grenzwert (Constraint) der Soft-Constraint Funktion } \\ \mathbf{A}_{\mathrm{BS}} & \text { Systemmatrix des Beispielsystems } \\ \mathbf{P} & \text { Gewichtungsmatrix der Gleichheitsnebenbedingung beim MPPC } \\ \mathbf{Q} & \text { Gewichtungsmatrix der Zustandsgrößen beim MPPC } \\ \mathbf{Q}_{\mathrm{LS}} & \text { Gewichtungsmatrix für die Lenksystemregelung } \\ \mathbf{Q}_{\mathrm{n}} & \text { Gewichtungsmatrix des Endwertes beim MPPC } \\ \mathbf{Q}_{\text {Ric }} & \text { Gewichtungsmatrix des Riccati-Reglers } \\ \mathbf{R} & \text { Gewichtungsmatrix der Stellgrößen beim MPPC } \\ \mathbf{R}_{\text {Ric }} & \text { Gewichtungsmatrix des Riccati-Reglers } \\ \mathbf{S} & \text { Gewichtungsmatrix der Änderung der Stellgrößen beim MPPC } \\ \mathbf{S}_{\mathrm{LS}} & \text { Gewichtungsmatrix für die Lenksystemregelung } \\ \mathbf{b}_{\mathrm{BS}} & \text { Eingangsvektor des Beispielsystems } \\ \mathbf{c}_{\mathrm{BS}} & \text { Ausgangsvektor des Beispielsystems } \\ \mathbf{e}_{\mathrm{n}} & \text { normaler Einheitsvektor } \\ \mathbf{e}_{\mathrm{t}} & \text { tangentialer Einheitsvektor } \\ \mathbf{f} & \text { (nichtlineare) mehrdimensionale Funktion } \\ \mathbf{g} & \text { Ungleichheitsnebenbedingung } \\ \mathbf{k}_{\mathrm{BS}} & \text { Verstärkungsvektor des Zustandsreglers des Beispielsystems } \\ \mathbf{u} & \text { Stellvektor } \\ \mathbf{x} & \text { Zustandsvektor } \\ \mathbf{x}_{\mathrm{BS}} & \text { reduzierter Zustandsvektor zur Kurswinkelregelung } \\ \mathbf{x}_{\lambda} & \\ \mathbf{x}_{\mathrm{v}} & \end{array}$




\section{Kurzfassung}

In kritischen Situationen sind viele Fahrer von PKWs mit der Fahrzeugführungsaufgabe überfordert. Die Unfallzahlen konnten bis 2013 auch durch die Einführung von aktiven Fahrerassistenzsystemen wie ABS, ASR und ESC gesenkt werden. In den folgenden Jahren ist ein leichter Anstieg zu verzeichnen. Um die Unfallzahlen wieder zu senken, werden neue Fahrerassistenzsysteme benötigt, die neben fahrdynamischen Größen auch Informationen über das Fahrzeugumfeld miteinbeziehen. Dies kann durch assistierende Funktionen, welche der Fahrer im Fehlerfall übersteuern kann, und/oder durch automatisierte Fahrfunktionen realisiert werden. Die Arbeit beschreibt und vergleicht vier verschiedene Verfahren zur Fahrzeugführung, die zur Kollisionsvermeidung im Straßenverkehr eingesetzt werden können. Das Bahnfolgeverfahren verwendet eine analytische Funktion zur Beschreibung der Ausweichbahn und eine Folgeregelung zur Führung des Fahrzeugs entlang der Bahn. Es ist ein einfaches Konzept, welches mit wenig Rechenleistung auskommt, sich aber nicht an viele verschiedene Situationen anpassen lässt. Deshalb wird das OnlineTrajektorienoptimierungsverfahren entwickelt. Zur Berechnung der Ausweichtrajektorien wird ein Gütemaß minimiert, welches Anteile zur Kollisionsvermeidung und zur Minimierung fahrdynamischer Reaktionen enthält. Die Realisierung der fortlaufend neu geplanten Trajektorie wird mit einer unterlagerten Geschwindigkeitsund Kurswinkelregelung durchgeführt. Das modellprädiktive Planungs- und Regelungsverfahren löst analog zum Online-Trajektorienoptimierungsverfahrens in jedem Abtastschritt ein Optimierungsproblem. Die kollisionsfreie Trajektorie wird zusätzlich an die Dynamikgleichungen eines Einspurmodells angepasst. Das Optimierungsproblem ist daher ein Optimalsteuerungsproblem, dessen Lösung neben der optimalen Trajektorie auch die zugehörigen Stellgrößen enthält. Die bisher getrennt behandelten Probleme, Trajektorienplanung und Folgeregelung, werden also in einem Schritt gelöst. Der Nachteil dieses Verfahrens ist der nochmals höhere Rechenaufwand im Vergleich zum Online-Trajektorienoptimierungsverfahren. Durch die Beschränkung auf konstante Stellgrößen während der Prädiktion und eine grobe Stellgrößendiskretisierung weist das modellprädiktive Trajektorienscharverfahren eine deutlich niedrigere Rechenlast auf. Die Vorteile der modellprädiktiven simultanen Planung und Regelung bleiben erhalten, jedoch können auf Grund des kurzen Prädiktionshorizontes weiter entfernte Hindernisse nicht in der Planung berücksichtigt werden. Durch die adaptive Wahl der

Diskretisierung wird auch im stationären Zustand eine hohe Regelungsgüte erreicht. Der abschließende Vergleich durch eine Nutzwertanalyse zeigt, dass die vier Verfahren, in Abhängigkeit des Anwendungsfalles, unterschiedlich gut geeignet sind. 



\section{1 \\ Einleitung}

\subsection{Motivation}

Fahrerassistenzsysteme und automatisierte Fahrfunktionen werden mit dem Ziel der Erhöhung des Fahrkomforts und/oder der Fahrsicherheit entwickelt. Mit dem Abstandsregeltempomaten (ACC - Adaptive Cruise Control) und dem Spurhalte- und Spurführungsassistenten (LGA - Lane Guidance Assist, LKA - Lane Keeping Assist) sind komfortorientierte Systeme entwickelt worden, welche auch einen Beitrag zur Fahrsicherheit leisten können. Jedoch werden zur Unterstützung des Fahrers in kritischen Situation andere Assistenzen benötigt: Während des Bremsmanövers wird der Fahrer durch ABS (Anti-lock Braking System) unterstützt, was ein Blockieren der Räder verhindert und so den Bremsweg durch bessere Ausnutzung des Kraftschlusspotentials verkürzt. Die Voraussetzung ist, dass der Fahrer stark genug bremst. Bei nicht ausreichender Betätigung des Bremspedals erhöht der Bremsassistent den Bremsdruck schneller als die meisten Fahrer. Zunächst wurden Systeme entwickelt, die durch die Auswertung der Gas- und Bremspedalstellung eine Notbremsung erkennen und infolgedessen aktiviert werden. Neuere Systeme verwenden zusätzlich Umfeldinformationen, welche durch Kameras und/oder Radare gewonnen werden. Über zunächst nur assistierend wirkende Systeme wurden bis heute bereits autonom eingreifende Funktionen (AEB - Autonomous Emergency Braking) für straßenzugelassene Fahrzeuge entwickelt. Bei der Querführungsaufgabe wird der Fahrer seit einiger Zeit durch Gierstabilisierungssysteme (ESC - Electronic Stability Control) unterstützt, welche das Fahrzeug mittels radselektivem Bremseingriff im fahrdynamischen Grenzbereich stabilisieren. Alle diese Systeme (ABS, ESC, AEB), zu denen auch die ASR (Antriebsschlupfregelung) und die MSR (Motorschleppmomentenregelung) zu zählen sind, haben zur Senkung der Unfallzahlen beigetragen, während die Marktdurchdringung gestiegen ist. Es gibt aber Situationen, in denen die vorgenannten Systeme nicht ausreichend helfen. Durch verschiedene elektronische Geräte werden Fahrer heute oft abgelenkt, sodass z.B. das Ende eines Staus übersehen wird. Nicht selten treten in derartigen Situationen Auffahrunfälle auf. Neben diesem Stauendeszenario führen Situationen, in denen Hindernisse unvorhergesehen auf der Fahrbahn erscheinen, häufig zu Kollisionen. Dies können beispielsweise Tiere oder Fußgänger sein, die zwischen zwei Fahrzeugen auf die Straße treten. Insbesondere Kinder oder alte Menschen sind dafür bekannt, die Fahrbahn unvorhersehbar und irrational zu betreten oder ihre Bewegungsrichtung zu ändern. Es können auch andere Fahrzeuge 
z.B. durch die Straßenrandbebauung oder -bepflanzung verdeckt sein und plötzlich auf die Straße fahren. Dies ist in Abbildung 1.1(a) in einem Landstraßenszenario dargestellt, während in Abbildung 1.1(b) das Stauendeszenario gezeigt wird. Es ist

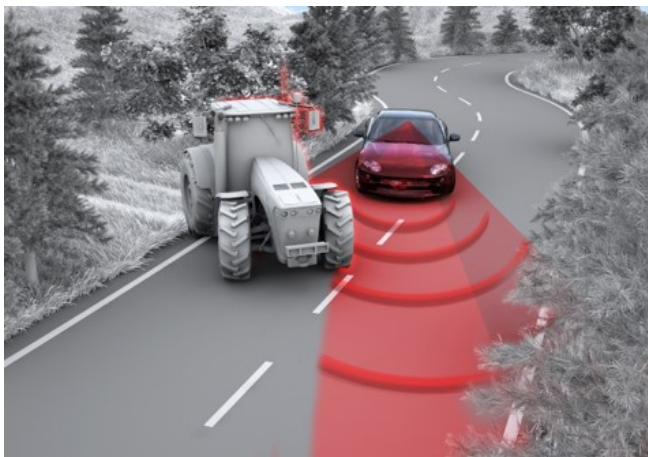

(a) Plötzlich auftretendes Hindernis.

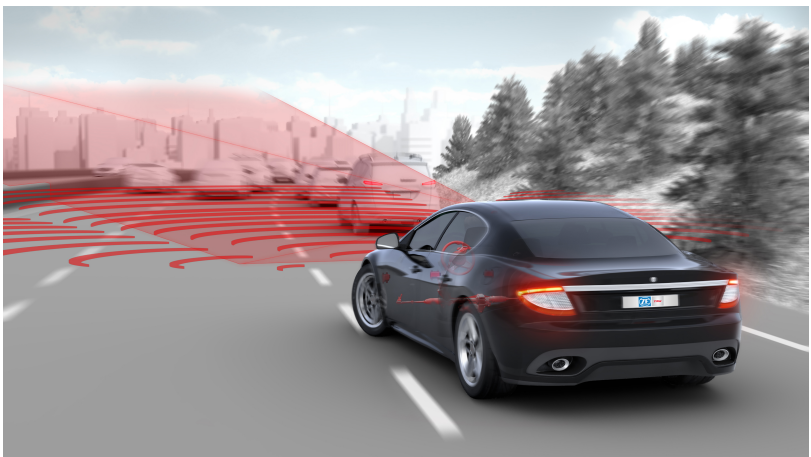

(b) Stauendeszenario.

Abbildung 1.1.: Typische Verkehrssituationen für ein Notmanöver zur Kollisionsvermeidung (Quelle: TRW Automotive GmbH).

bekannt, dass der Fahrer in dieser Situation häufig Bremseingriffe und/oder Lenkbewegungen ausführt, welche das Fahrzeug destabilisieren und/oder in einer Kollision enden. In diesen Situationen sind Fahrerassistenzsysteme wie Adaptive Cruise Control (ACC) oder Lane Guidance/Keeping nicht in der Lage einen Unfall zu vermeiden. Je nach Situation ist dies auch mit der AEB nicht möglich. Zwar unterstützen die Fahrstabilisierungssysteme ABS und ESC den Fahrer bei einem Notmanöver, jedoch werden die Umfeldinformationen nicht berücksichtigt und es werden nur Fehler des Fahrers korrigiert. Er erhält aber keine Rückmeldung dahingehend, welche Bremsund Lenkeingaben optimal oder zumindest angebracht sind. Die entwickelten Systeme der aktiven Sicherheit reichen nicht aus und es bedarf eines weiteren Fahrerassistenzsystems. Es ist daher sinnvoll, dem Fahrer durch Überlagerung eines Lenkmomentes, welches zusätzlich zur normalen Servofunktion mit dem elektromechanischen Lenksystem aufgebracht wird, aufzuzeigen, welcher Lenkradwinkel in der jeweiligen Situation angemessen ist (ESA - Emergency Steering Assist). Gleichzeitig greift die Assistenz in die Längsführung ein, um das volle Kollisionsvermeidungspotential des Fahrzeugs auszuschöpfen. Um eine Kollision zu vermeiden, gibt es zwei prinzipiell unterschiedliche Möglichkeiten: Notbremsung oder Notausweichmanöver. Dabei wird das Notausweichmanöver weiter unterteilt in ein reines Lenkmanöver und das kombinierte Lenk- und Bremsmanöver. Obwohl das Ausweichmanöver deutlich schwieriger und unsicherer in Bezug auf unvorhergesehene Hindernisse ist, kann in bestimmten Situationen ein Ausweichmanöver, verglichen mit einem Notbremsmanöver, zu einem späteren Zeitpunkt erfolgreich durchgeführt werden. [Sch07] und [Stä08] vergleichen hierzu Brems- und Ausweichweg in einer Situation mit einem Hindernis, welches die Fahrspur des Ego-Fahrzeugs blockiert, und einem zweiten freien Fahrstreifen, auf den ein Spurwechsel durchgeführt werden soll. Bei höheren Differenzgeschwindigkeiten (ab ca. $50 \mathrm{~km} / \mathrm{h}$ ) liegt demnach der last-point-to-steer hinter dem last-point-to-brake. Neben der Entwicklung einer Assistenzfunktion zur Unterstützung eines Menschen am Steuer gibt es mit den automatisierten Fahrfunktionen ein weiteres Einsatzgebiet 
für Funktionen zur Kollisionsvermeidung. Bei einer Assistenzfunktion muss der Fahrer ein Notausweichmanöver durch eine deutliche Lenkbewegung einleiten und er muss in der Lage sein, die Lenkempfehlungen zu übersteuern. Um dem Fahrer Empfehlungen geben zu können, muss zunächst ein System entwickelt werden, welches das Manöver theoretisch auch autonom durchführen könnte. Das Hauptaugenmerk liegt dabei auf der Kollisionsvermeidung und der Minimierung fahrdynamischer Reaktionen, während andere Anforderungen nach komfortabler Fahrweise, Energieeffizienz und Zeitoptimalität ausgeblendet werden müssen. Aufgrund der großen Unsicherheit in der Umfelderfassung hat eine solche autonome Funktion zur Zeit keine praktische Relevanz. Sobald es dem Fahrer im Zuge der Einführung automatisierter Fahrfunktionen erlaubt wird, sich fahrfremden Tätigkeiten zu widmen, ist eine autonome Kollisionsvermeidung jedoch zwingend erforderlich.

\subsection{Grundaufgaben eines Kollisionsvermeidungssystems}

Ein Kollisionsvermeidungssystem besteht aus den in Abbildung 1.2 gezeigten Grundaufgaben. Die Situationserfassung beinhaltet sowohl die Umfelderfassung als auch die

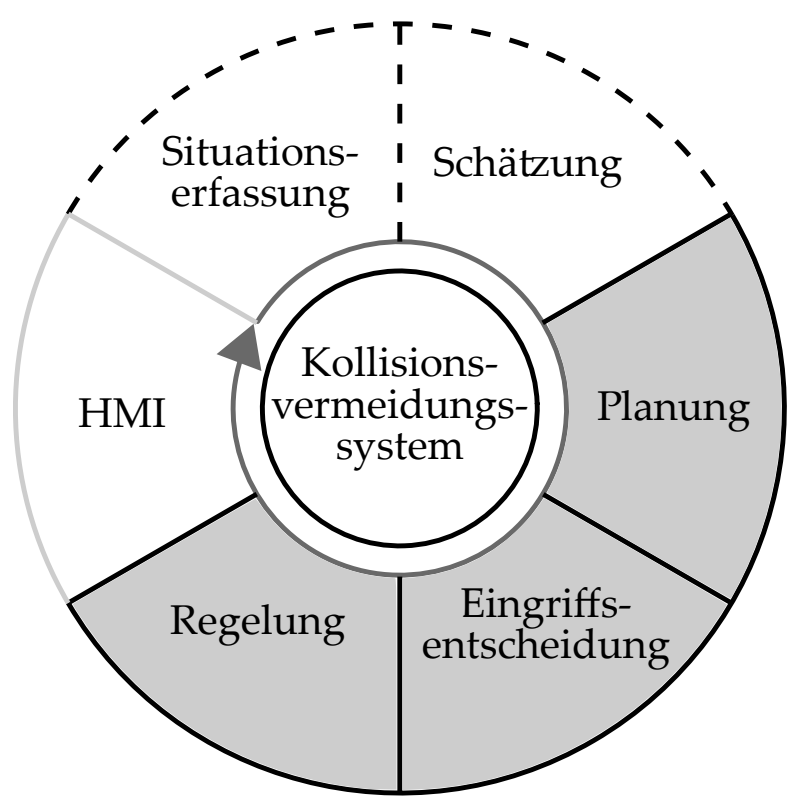

Abbildung 1.2.: Grundaufgaben eines Kollisionsvermeidungssystems.

Messung der fahrdynamischen Größen. Basierend auf diesen Informationen wird mit Schätzungsalgorithmen die Entwicklung der Verkehrssituation prädiziert und nicht messbare Größen werden mit Beobachterstrukturen rekonstruiert. Darauf aufbauend erfolgt die Planung von kollisionsfreien Trajektorien bzw. Bahnen. Durch die Analyse der Trajektorie bzw. Bahn wird dann eine Entscheidung über einen Eingriff und die Art des Eingriffs getroffen. Im Falle einer Assistenzfunktion müssen zusätzlich die Fahrereingaben berücksichtigt werden. Ist die Entscheidung zum Eingriff gefallen, so muss mit einer Regelung die Trajektorie realisiert werden. Gleichzeitig muss der Fahrer unter Ausnutzung mehrerer Kanäle (akustisch, visuell, haptisch) gewarnt, informiert 
und instruiert werden (HMI). Diese Arbeit fokussiert auf die Planung, Eingriffsentscheidung und Regelung. Das Themengebiet der Situationserfassung und der Schätzung werden nicht betrachtet. Vielmehr wird angenommen, dass alle Messgrößen ideal erfasst und alle Hindernistrajektorien exakt bekannt sind. Das HMI wird teilweise diskutiert.

\subsection{Stand der Forschung und Technik}

Dieser Abschnitt benennt zunächst Veröffentlichungen, welche durch Unfallstatistiken und Nutzenanalysen die Notwendigkeit von Kollisionsvermeidungssystemen aufzeigen. Darauf folgend wird der Notausweichassistent in die Entwicklung der Fahrerassistenzsysteme eingeordnet, bevor Varianten und Ausprägungsstufen der Systeme diskutiert werden. Anschließend wird die Fachliteratur bezüglich der drei Grundaufgaben Planung, Eingriffsentscheidung und Regelung analysiert. Im Bereich der Trajektorienplanung wird zunächst der Technologietransfer von der mobilen Robotik, in der leistungsfähige Planer bereits seit langer Zeit Forschungsgegenstand sind, in den Automotive-Sektor aufgezeigt. Anschließend werden die Planungsverfahren für automatisierte/autonome Fahrzeuge vorgestellt, bevor solche Verfahren, die einen Bezug zur Kollisionsvermeidung in Notsituationen haben, in die Bereiche analytische Funktionen, Trajektorienoptimierung und modellprädiktive Verfahren eingeordnet werden. Die Publikationen zur Eingriffsentscheidung und Manöverwahl werden in die regelbasierten und die situationsangepassten Verfahren unterteilt. Dann wird die Literatur zu Fahrdynamik- und Positionsregelungen mit dem Ziel der Kollisionsvermeidung vorgestellt. Abschließend wird die Wirksamkeit von assistierenden Kollisionsvermeidungssystemen in Probandenstudien aufgezeigt.

\section{Unfallstatistiken und Nutzenanalyse}

Eine Analyse von Unfalldaten (siehe [Eid+07]) zeigt, dass durch die Entwicklung von Kollisionsvermeidungssystemen eine Vielzahl von Unfalltypen addressiert werden. Hierzu wird betrachtet, welcher Unfalltyp durch welches Assistenzsystem potentiell verhindert werden könnte, und zusätzlich, welchen Aufwand bzw. welche Kosten die Entwicklung und Produktion verursachen würden. Der Aussage in [Kae+09] zufolge können bereits mit einem AEB System ca. $63 \%$ der Unfälle adressiert werden. Weber [Web12] zufolge sind 90\% aller registrierten Unfälle auf Fehlverhalten des Fahrers zurückzuführen. Zudem wird das Wirkfeld 11 einer Bremsausweichassistenz mit 14,2\% und der Wirkgrad ${ }^{2}$ mit 8,93\% (bei autonomem Eingriff) auf Basis der Daten der German In-Depth Accident Study abgeschätzt. Zum Vergleich wird das ESC mit einem Wirkfeld von $9 \%$ und einem Wirkgrad von $7 \%$ bewertet. Laut Statistischem Bundesamt sind $67 \%$ aller Unfälle mit Personenschaden auf den Fahrer zurückzuführen (siehe [Des15]). Zusammenfassend kann festgestellt werden:

- Es geschehen zu viele Unfälle.

- Das Fehlverhalten der Fahrer ist eine der Hauptunfallursachen.

\footnotetext{
${ }^{1}$ Anteil des Unfalltyps an allen Unfällen

${ }^{2}$ Anteil der mit dieser Assistenz vermeidbaren Unfälle
} 
- Die etablierten Fahrerassistenzsysteme können zu einer weiteren Reduktion der Anzahl der Unfälle keinen entscheidenden Beitrag leisten.

- Mit einem Kollisionsvermeidungssystem lassen sich viele weitere Unfälle potentiell verhindern.

\section{Entwicklung der Fahrstabilisierungs- und Fahrerassistenzsysteme}

Die Entwicklung der Fahrerassistenzsysteme begann mit der Einführung von ABS, ASR, MSR und ESC, welche auch als Fahrstabilisierungssysteme bezeichnet werden. Diese Systeme dienen der Sicherheit und nicht dem Komfort. Zur Realisierung wurden Sensoren eingesetzt, welche fahrzeuginterne Messgrößen erfassen und nur wenig Unsicherheit aufweisen. Die Verringerung der Unfallzahlen ist (neben anderen Faktoren wie z.B. den passiven Sicherheitssystemen oder auch gesetzlichen Regelungen wie der Gurtpflicht oder der Senkung der Alkoholgrenzen) auch diesen ersten Systemen der aktiven Sicherheit zu verdanken (siehe [Meh15]). Mit der ausschließlichen Messung interner Fahrdynamikgrößen lassen sich aber nicht alle Unfälle verhindern. In diesem Zusammenhang zogen umfelderfassende Sensoren in die Fahrzeuge ein. Eine große Verbreitung haben dabei Kamera und Radarsensoren gefunden. Kameras werden häufig zur Spurhaltung und Spurführung eingesetzt. Mit Radaren wurden zunächst Komfortfunktion wie ACC realisiert. Später kam mit der AEB eine Sicherheitsfunktion hinzu, welche häufig mit fusionierten Daten der beiden Sensorsysteme arbeitet. Bei der Gestaltung der Bremsassistenten gibt es mehrere Ausprägungsstufen, sodass entweder der Bremsdruck erhöht wird, wenn der Fahrer bremst, oder (im Falle der AEB) autonom eine Vollbremsung durch die Assistenz eingeleitet wird. Aber auch diese Funktion kann nicht alle Kollisionen vermeiden. Zum einen ist dies in der begrenzten "sicheren" Reichweite ${ }^{3}$ der Umfeldsensoren begründet, zum anderen soll ausgeschlossen sein, dass der Fahrer selbst die Kollision noch durch ein Ausweichmanöver verhindern kann (siehe [Eid11]). Als Ergebnis kann (insbesondere bei höheren Differenzgeschwindigkeiten) in vielen Situationen lediglich eine Verringerung der Unfallschwere erreicht werden. Mit einem kombinierten Brems- und Lenkmanöver können Kollisionen in vielen Situationen noch vermieden werden. Für ein stehendes Hindernis gibt es in der Literatur vielfach kinematische Abschätzungen, welche Art von Manöver zu einem späteren Zeitpunkt kollisionsfrei durchführbar ist (siehe [Stä08; Sch07; Mos+12; Has+12, Web12]). Der Nachteil eines Ausweichmanövers ist die deutlich höhere Komplexität hinsichtlich der Umfelderfassung, Planung, Entscheidungsfindung und Durchführung im Vergleich zum ausschließlichen Bremsmanöver. Es ist allgemein bekannt, und auch in Studien belegt (z.B. [Kel+14a; Meh+10; Sie+15]), dass der Normalfahrer Notmanöver in der Regel nicht beherrscht, da diese das Fahrzeug in den fahrdynamischen Grenzbereich bringen, in welchem der Normalfahrer mit der Fahrzeugreaktion nicht vertraut ist. Die Entwicklung einer Assistenz zur Vermeidung von Kollisionen in Notsituationen ist daher der nächste Schritt auf dem Weg der Vision des unfallfreien Fahrens.

${ }^{3}$ Damit ist die Reichweite gemeint, in welcher Hindernisse mit geringer Unsicherheit erkannt und klassifiziert werden. 


\section{Varianten von Kollisionsvermeidungssystemen}

Aus der Literatur sind unterschiedliche Varianten von Kollisionsvermeidungssystemen bekannt. Zum einen gibt es die bereits in Serienfahrzeugen vorhandenen Notbremssysteme und zum anderen Notausweichsysteme. Im Allgemeinen besteht ein Ausweichmanöver aus Lenk- und Bremseingriffen. Unterschiede finden sich bei der Gestaltung der Schnittstelle zum Lenksystem. Es besteht die Möglichkeit, mit einer Überlagerungslenkung unabhängig vom Fahrer zusätzliche Lenkwinkel zu stellen (siehe z.B. [Sch+06b; Sch07; Sch+06c; Stä08]) oder mit einer elektromechanischen Servolenkung Lenkmomente aufzubringen (siehe z.B. [Kel+14a; Kel+15b; See+15; Cho+14; Iwa+14; Kat+14; Sie+15]). Die Eingriffe einer Überlagerungslenkung nehmen dem Fahrer die Möglichkeit, das System im Fehlerfall zu übersteuern. Werden zusätzliche Lenkmomente gestellt, wird das Manöver von der Assistenz und dem Fahrer gemeinsam durchgeführt (Shared Control), wobei durch die Schnittstelle sicher gestellt wird, dass der Fahrer stärker ist und das System übersteuern kann. Anders als bei der Shared Control Sichtweise kann der Fahrer auch als Störgröße angesehen werden, da er ebendiese Art von Fahrzeugführung nicht beherrscht. In diesem Fall kann der Störeinfluss durch den Fahrer mit einer Überlagerungslenkung besser unterdrückt und im Idealfall eliminiert werden. In [Del+10] wird vorgeschlagen, statt einer Drehmomentüberlagerung, eine Impedanzregelung mit der EPS durchzuführen und die Trägheit, Dämpfung und Steifigkeit der Lenkung im Sinne der Kollisionsvermeidung anzupassen. Neben dem Lenkeingriff werden häufig zusätzliche Bremseingriffe vorgenommen, wobei [Cho+14; Ack+15; Göt14] auch radselektive Eingriffe betrachten. Neben der Aktorkonfiguration gibt es Unterschiede bei der Auslösung eines Notmanövers. Entweder kann es zur Bedingung gemacht werden, dass der Fahrer mit einer deutlichen Lenkbewegung das Manöver einleiten muss, oder das Assistenzsystem löst basierend auf einem Kritikalitätsmaß den Eingriff aus. Häufig wird der Auffahrunfall als Szenario betrachtet. In [Eid+07] wird der Fahrer bei Spurwechselmanövern unterstützt, wenn eine Kollision droht. [Kat+14] schlägt eine Assistenz vor, welche in kritischen Situationen das Abkommen von der Fahrbahn verhindert. [Sat+08] betrachtet eine Kreuzungssituation und [Ito+12] und [Kel+11] vermeiden Kollisionen mit Fußgängern. Die meisten Veröffentlichungen behandeln PKWs während [Has+12] auch für LKWs Notausweichmanöver vorschlägt. [Web12] diskutiert die Möglichkeit, mit einem großen Head-up-Display Fahrempfehlungen in der Windschutzscheibe grafisch aufzuzeigen.

\section{Trajektorienplanung}

Technologietransfer aus der mobilen Robotik Innovative Bahn- und Trajektorienplaner werden bereits seit einiger Zeit in der mobilen Robotik verwendet. Die Verwendung eines elastischen Bandes, welches sich mittels Optimierung an seine Umgebung anpasst, wird von [Qui+93] vorgeschlagen. Der Ansatz wurde mehrfach zur Fahrzeugführung angewendet und erweitert. In [Geh+01] wird ein Abstandsregeltempomat um eine Querführung mit elastischen Bändern ergänzt, um Hindernissen lokal auszuweichen und das Folgeverhalten zu verbessern. In [Hil+03] und [Hil05] wird eine autonome Querführung zur Spurhaltung und Kollisionsvermeidung eingesetzt. In [Sat+05; Bra+07; Bra07] wird das Verfahren ebenfalls eingesetzt, wobei das Umfeldmodell aus einem Potentialfeld besteht, welches als Gefahrenkarte besetzte Verkehrsflächen 
beschreibt. Obwohl sich mit diesem Verfahren gute Ergebnisse erzielen lassen, werden Bahnen berechnet, welche keine Zeitinformationen enthalten. Dadurch bedingt muss die Längsführungsaufgabe getrennt gelöst werden. Dieser Nachteil wird bei dem wiederum aus der mobilen Robotik stammenden Verfahren der gezeiteten elastischen Bänder (Timed Elastic Bands - TEB) gelöst. Das Verfahren wird in [Rös+12; Rös+13; Rös+15a] entwickelt und erlaubt die kollisionsfreie Quer- und Längsführung eines mobilen Roboters unter Berücksichtigung mehrerer Gütekriterien und Beschränkungen. Die Problemformulierung mit weichen Nebenbedingungen lässt eine effiziente Lösung des Optimierungsproblems zu. In [Kel+14b] und in Kapitel 5 wird das TEB zur kombinierten Fahrzeugführung in Notsituationen eingesetzt.

Trajektoriengenerierung für automatisiertes Fahren Um automatisierte Fahrfunktionen zu realisieren, werden leistungsfähige Bahn- bzw. Trajektorienplaner benötigt. In der Literatur sind mehrere Ansätze veröffentlicht, über die im Folgenden ein kurzer Überblick gegeben werden soll. Grundlagen zur Fahrzeugführung sind bereits im PROMETHEUS Programm erarbeitet worden. In [Ulm94] wird ein automatisiertes Fahrzeug (VITA II) beschrieben, welches bereits 1994 eine längere Fahrstrecke autonom zurückgelegt hat. Große Aufmerksamkeit wurde bei der DARPA Urban Challenge erreicht. In diesem Wettbewerb sind mehrere Teams gegeneinander angetreten und die verwendeten Algorithmen der Trajektorienplanung wurden veröffentlicht. Eine umfangreiche Beschreibung eines der Teams findet sich z.B. in [Mon+08]. Ein ähnlicher Wettbewerb in China wird von [ [Fu+15] beschrieben. Die Verfahren lassen sich wie folgt kategorisieren:

- State Lattices: In [Zie+09] als auch in [ $[\overline{\mathrm{McN}+11}]$ werden Trajektorienplaner vorgestellt, welche auf einem diskreten Zustandsgitter basieren und recheneffizient implementiert werden können. Die Ergebnisse in [McN+11] sind vielversprechend für das automatisierte Fahren. Die grobe Zustandsdiskretisierung lässt, verglichen mit den anderen unten genannten Verfahren, auf eine geringere Präzision bei der Fahrzeugführung schließen. Die Eignung zur Kollisionsvermeidung ist daher fragwürdig.

- Optimierung kinematischer Größen: Die in [Zie+08; Cho+10; Zie+14b; Zie+14a; Hom15; Hom+17] vorgestellten Verfahren basieren auf dem Massenpunktmodell und minimieren kinematische oder geometrische Größen. In [Zie+14b] und [Zie+14a] wird die sogenannte Bertha Benz Fahrt beschrieben. Dabei fuhr ein automatisiertes Fahrzeug eine längere Strecke über verschiedene Straßentypen und im dichten Verkehr.

- Modellprädiktive Verfahren: In [Pre+01; And+09; Yoo+09; Sch+11; And+12; Sch+12; Göt+15: Göt+16a; Thi15] werden dynamische Modelle in die Planung integriert, um diese genauer zu gestalten und die Realisierbarkeit der Trajektorie zu gewährleisten. In einigen Fällen können die Stellgrößen direkt im Sinne einer modellprädiktiven Regelung auf die Regelstrecke angewendet werden. In anderen Fällen (siehe [YYoo+09]) werden zusätzlich unterlagerte Trajektorienfolgeregler eingesetzt. 
- Trajektorienscharverfahren: In [Hun+08] und [Wer+10] werden scharbasierte Verfahren vorgestellt. Hierbei wird jeweils aus einer diskreten Menge von möglichen Trajektorien diejenige ausgewählt, welche den geringsten Wert eines Gütemaßes liefert.

Die Berücksichtigung von Hindernissen bei der Trajektorienplanung wird häufig mit Umfeldmodellen realisiert. In den obigen Veröffentlichungen werden verschiedene Möglichkeiten aufgezeigt. Die Entwicklung eines umfangreichen Umfeldmodells für das automatisierte Fahren wird in [Wan+15] beschrieben. Ein Vergleich zwischen drei Trajektorienplanern wird in [Mad+13] vorgestellt. Ein umfassender Überblick über Trajektorienplaner zum automatisierten Fahren findet sich in [Kat+15].

Trajektorienplanung zur Kollisionsvermeidung in Notsituationen Aus der Literatur sind mehrere Ansätze zur Berechnung von Kollisionsvermeidungstrajektorien bekannt, welche sich in die drei Gruppen: Analytische Funktionen, Bahn- bzw. Trajektorienoptimierung und modellprädiktive Verfahren einteilen lassen.

- Analytische Funktionen: Die Verwendung von analytischen Funktionen zur Beschreibung von Ausweichbahnen ist weit verbreitet. In einigen Fällen wird eine Sigmoidfunktion verwendet (siehe [Sch+06b; Sch+06c; Sch07; Stä08; Cho+11; Ack+14]). Der Nachteil der Sigmoide ist, dass sie an den Übergangsstellen zur Geradeausfahrt unstetig ist. Die Sigmoide kann zwar so gewählt werden, dass sich diese Unstetigkeit quasi nicht bemerkbar macht, jedoch hat dies Auswirkungen auf den restlichen Verlauf. Häufig werden daher Polynome unterschiedlichen

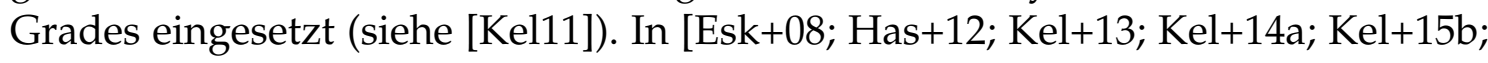
Buß13: See+15] werden Polynome 5. Grades, in [Kel+11] Polynome 7. Grades verwendet, um kollisionsfreie Bahnen zu planen. [Shi+10] und [Shi+12] bestimmen zwei Polynome 6. Grades für die Quer- und Längsplanung, um Trajektorien für einen Doppelspurwechsel zu planen. Eine andere Vorgehensweise wird in $[\widehat{S o u+11]}$ und [Cho+14] vorgestellt. Ausgehend von einem Querbeschleunigungsprofil wird eine Ausweichbahn berechnet. [Bev08] und [Hay+12] verwenden mehrere Kreisbögen und Geradenstücke, um eine Ausweichbahn zusammenzusetzen. In [Ack+14] wird ein Sollwertsprung (der Sollwert wird sprungförmig von der aktuellen Spur in die benachbarte Spur verlegt) mit einem linearen System 4. Ordnung gefiltert. Alle vorgenannten Verfahren haben den Vorteil, mit nur geringem Rechenaufwand kollisionsfreie Bahnen zu liefern, welche aus fahrdynamischer Sicht zwar nicht optimal aber vorteilhaft verlaufen. Der Nachteil besteht darin, dass meist nur einfache Verkehrssituationen gelöst werden können.

- Bahn- bzw. Trajektorienoptimierung: Deutlich anpassungsfähiger sind solche Verfahren, die online eine Trajektorie optimieren und diese so an die aktuelle Situation anpassen. Zu unterscheiden sind Bahnplaner, wie sie in [Hil+03; Sat+05; Bra+07; Bra07; Sat+08] vorgestellt werden, und Trajektorienplaner. Mit einem Bahnplaner können keine kombinierten Brems- und Lenkmanöver geplant werden. Die durch die Bahnplanung bestimmte Querführung muss durch eine separate Längsregelungsstrategie ergänzt werden. Bei einem Trajektorienplaner werden Quer- und Längsführung in einem Verfahren bestimmt, sodass die 
Kopplung zwischen den Bewegungsrichtungen zumindest tendenziell Berücksichtigung findet. In [Sch+06a] und [Sch13] werden mögliche Aufenthaltsorte für Hindernisse berechnet und basierend darauf eine kollisionsfreie Trajektorie mit minimaler Krümmung bestimmt. Um die Trajektorie durch einen standardisierten Doppelspurwechsel zu optimieren, wird in [Bev08] und [Bev+10] ein dreistufiger Optimierungsprozess vorgeschlagen, welcher Vorteile gegenüber einem einfachen geometrischen Bahnplanungsverfahren aufweist. Die Kombination aus einem Trajektorienplaner mit modellprädiktivem Folgeregler wird in [Gao+12] vorgestellt. Damit gelingt es, Szenarien mit einem oder zwei stehenden Hindernissen zu umfahren. In [Gor+14] wird ein Trajektorienplaner vorgeschlagen, welcher zeitoptimale Spurwechseltrajektorien berechnet. Weitere auf der Minimierung kinematischer Größen basierende Trajektorienplaner werden in [Muk+14; Hom13; Hom15; Hom+17; Kel+14b; Fer+16] präsentiert.

- Modellprädiktive Verfahren: Der Unterschied zwischen den kinematischen Trajektorienplanern und den modellprädiktiven Verfahren ist, dass bei den modellprädiktiven Verfahren ein dynamisches Modell berücksichtigt wird. Die modellprädiktiven Verfahren lassen sich weiter unterteilen in die modellprädiktiven Planungsverfahren, welche einen zusätzlichen Fahrdynamik- bzw. Positionsregler zur Realisierung der Trajektorie benötigen, und modellprädiktive Planungsund Regelungsverfahren, welche neben der optimalen Trajektorie auch die optimalen Stellgrößen berechnen. Um unvermeidbare Kollisionen zu erkennen, wird in [Kar08] ein modellprädiktives Planungsverfahren verwendet. Liefert das Verfahren keine realisierbare Lösung, so wird die Kollision als unvermeidbar eingestuft und eine Notbremsung zur Reduktion der Unfallschwere ausgelöst. In [Din+10] wird ein Optimalsteuerungsproblem gelöst, wobei die Reifenkräfte als fiktive Stellgrößen verwendet werden. Das Ausweichmanöver wird dabei in drei Phasen aufgeteilt, was die Allgemeingültigkeit bei mehreren bewegten Hindernissen einschränkt, da sich unter Umständen keine drei Phasen erkennen lassen. Ein Vergleich zwischen einer optimalen Trajektorie, basierend auf dem linearen Einspurmodell, und einem Polynom 5. Grades wird in [Sou+10] präsentiert. Aufgrund der unterschiedlichen Randbedingungen hat der Vergleich jedoch nur eine begrenzte Aussagekraft. In [Bau+12] und [Bau+13] wird die Potentialfeldmethode mit der Optimalsteuerungstheorie verknüpft. Basierend auf einem nichtlinearen Einspurmodell werden optimale Lenkmanöver berechnet, welche Hindernisse in Form von Potentialfeldern berücksichtigen. In [Wer+12] wird ein vereinfachtes Modell der Fahrzeugdynamik verwendet, welches gegenüber einem Einspurmodell den Vorteil hat, keine Singularität im Stillstand aufzuweisen. Allerdings ist die Genauigkeit des Modells geringer, sodass unterlagerte Positionsregler zur Realisierung der Trajektorie verwendet werden müssen. Um den Fahrer zu unterstützen, wird ein modellprädiktiver Planungs- und Regelungsansatz in [And+09] und [And+12] präsentiert, welcher auf einem linearen Einspurmodell basiert. Da das Prädiktionsmodell die Gültigkeit im Grenzbereich verliert, ist die Eignung für Notmanöver fraglich. [Fra+13] verwendet ein detailliertes Zweispurmodell, was wiederum aufgrund des hohen Rechenaufwandes Nachteile aufweist, zumal die Ergebnisse nur geringe Bremseingriffe zeigen. In [Göt14] und [Göt+15] wird 
ein vereinfachtes Zweispurmodell verwendet und die Möglichkeit des radselektiven Bremseingriffes untersucht. Es wird gezeigt, dass das Verfahren auch für automatisierte Fahrfunktionen anwendbar ist. In [Thi15] und [Göt+16a] wird hingegen ein nichtlineares Einspurmodell verwendet und es werden lediglich Lenkmanöver betrachtet. Es gelingt das Verfahren für eine Echtzeitanwendung zu implementieren und verschiedene kritische Verkehrssituationen zu untersuchen. Nach leichter Modifikation wird das Verfahren auch zur automatischen Querführung auf einer Rennstrecke eingesetzt. Die echtzeitfähige Implementierung modellprädiktiver Verfahren ist aufgrund des hohen Rechenaufwandes eine große Herausforderung. Zur Reduzierung des Rechenaufwandes schlägt [Yi+16] vor, das nichtlineare Prädiktionsmodell an verschiedenen Arbeitspunkten zu linearisieren. So können kombinierte Lenk- und Bremsmanöver durchgeführt werden. Trotz möglicher Vollbremsung wählt das Verfahren jedoch ein Ausweichmanöver, obwohl das Bremsmanöver in dieser Situation einfacher durchführbar wäre. Eine andere Möglichkeit, den Rechenaufwand und auch die Komplexität der Verfahren deutlich zu senken, ist das modellprädiktive Trajektorienscharverfahren. Aufgrund einer groben Diskretisierung und der Beschränkung auf konstante Stellgrößen während der Prädiktion wird die Echtzeitfähigkeit leicht nachweisbar, wie in [Har14; Kel+15a; Kel+15c] gezeigt wird. Die mit der Vereinfachung einhergehende Suboptimalität zeigt keinen großen Einfluss auf das Regelungsergebnis. Das Verfahren lässt sich jedoch aufgrund der Beschränkung auf konstante Stellgrößen während der Prädiktion nicht in dem Sinne erweitern, dass ein globales Optimum gefunden wird.

\section{Eingriffsentscheidung und Manöverwahl}

Aus der Literatur sind Verfahren bekannt, welche für ein AEB System sicher stellen, dass eine Kollision unvermeidbar ist. Dies wird zum einen in [Eid11] und zum anderen auch in [Kar08] gezeigt, wobei das letztgenannte Verfahren auch zur Berechnung von Ausweichmanövern geeignet ist. Hinzu kommen Verfahren, welche zum einen eine Entscheidung treffen, ob ein Kollisionsvermeidungsmanöver eingeleitet wird, und zum anderen welche Art von Manöver (Bremsmanöver, Lenkmanöver oder eine Kombination aus beiden) zu präferieren ist. Hierzu kann die Literatur wiederum in zwei Gruppen unterteilt werden:

- Regelbasierte Entscheidung: Bei dieser Art der Entscheidung wird anhand von einfachen Regeln ein Manöver ausgewählt (siehe [Kel+11; Sou+11; Tam+11; Hay+12; Ise+12; Mil+12; Ack+14; Kel+14a; Kel+15b]). Ein Beispiel für eine solche Regel wäre: „Bei Geschwindigkeiten unterhalb eines Grenzwertes ist ein Bremsmanöver auszuführen“. Diese Regel ist eine geeignete Wahl für ein stehendes Hindernis in der eigenen Spur, welches diese Spur vollständig blockiert. Wenn aber zusätzlich ein weiteres schnelles Fahrzeug hinter dem Ego-Fahrzeug fährt, kann dies eine ungeeignete Entscheidung darstellen. Eine weitere Regel könnte lauten: „Wenn in der eigenen Spur ein Hindernis steht und die benachbarte Spur durch ein überholendes Fahrzeug belegt ist, dann soll ein Bremsmanöver ausgeführt werden". Auch diese Regel wird in bestimmten Situationen eine geeignete Wahl darstellen. Es stellt sich jedoch die Frage, wann genau ein Fahr- 
streifen belegt ist. Fährt beispielsweise das Fahrzeug auf der angrenzenden Spur an dem einen Rand und das Fahrzeug in der Spur des Ego-Fahrzeugs an dem gegenüberliegenden Rand, so kann dazwischen ausreichend Freiraum für ein Ausweichmanöver vorhanden sein, wodurch die obige Regel ihre Allgemeingültigkeit verliert. Eine Möglichkeit diesen Problemen zu begegnen ist es, genauere oder bessere Regeln zu entwerfen. Jedoch erscheint es angesichts dessen, dass im realen Straßenverkehr nahezu beliebig komplexe Situationen entstehen können, nicht zweckmäßig dieser Herausforderung mit immer mehr und komplexeren Regeln entgegenzutreten, zumal die Regeln auf Konsistenz zu überprüfen sind.

- Situationsangepasste Entscheidung: Bei diesen Verfahren wird eine Trajektorie mittels Optimierung an die jeweilige (beliebige) Situation angepasst (siehe [Kel+14b; Göt+15; Kel+15a; Göt+16a; Göt+16b; bietet die Möglichkeit, die Kritikalität einer Situation nach Definition A.1.14 zu approximieren. Auf Basis der Kritikalität kann dann eine einheitliche Entscheidung getroffen werden, ob ein Notmanöver auszuführen ist. Zudem ist das Verhältnis von Quer- und Längsführung bereits in der optimalen Trajektorie enthalten, sodass hier keine weitere diskrete Entscheidung nötig ist.

\section{Fahrdynamik- und Positionsregelung}

Fahrdynamik- und Positionsregler für den querdynamischen Grenzbereich sind aus den Anwendungsfeldern Notausweichen und Fahren auf Rennstrecken bekannt. Es sind verschiedene Ansätze bekannt, welche sich wie folgt gliedern lassen:

- PID ähnliche Regelungsstrukturen: PID-Regler und ähnliche Reglerstrukturen werden häufig für Bahn- und Trajektorienfolgeregler verwendet (siehe [Sch+06c; Sch07; Bev08; Esk+08; Tal+11; Ack+14; Iwa+14; Ack+15; Kel+15b]). Hierbei werden sowohl kaskadierte (z.B. [Kel+15b]) als auch parallele Regelkreisstrukturen (z.B. [Tal+11]), bei denen sowohl die Position als auch die Gierrate parallel geregelt werden, eingesetzt. Der Vergleich in [Sch07] bewertet einen geschwindigkeitsadaptiven PD-Regler mit Vorsteuerung als ähnlich leistungsfähig wie aufwändigere Zustandsregelungen.

- Zustandsregler: Neben den PID-ähnlichen Reglern werden häufig Zustandsregler eingesetzt (siehe [May91; Smi+95; Sch+06b; Sch+06c; Kön+07; Sch07; Esk+08; Kön09: Men+14]).

- Fuzzy Regler: In [Wei03] und [Fer+11] werden Fuzzy Regler zur Kollisionsvermeidung vorgeschlagen. In beiden Fällen ergeben sich geschwindigkeitsadaptive Regler (gesteuerte Adaption).

- Spezielle Regler: In [Keh+06; Keh07; Keh+07] wird ein nichtlinearer Zustandsregler mit einem PD-Regler ergänzt. Das Konzept wird von [Kel+13; Kel+14a; See+15] übernommen, während [Kön09] einen Sliding Mode Regler untersucht.

- Modellprädiktive Regelung: Die Verwendung eines modellprädiktiven Reglers als Bahn- bzw. Trajektorienfolgeregler ist von den weiter oben erwähnten modellprädiktiven Planungsverfahren und den modellprädiktiven Planungs- und 
Regelungsverfahren zu unterscheiden. Aufgrund der vielseitigen Einsatzmöglichkeit und der einfachen Berücksichtigung von Nebenbedingungen werden modellprädiktive Regler häufig zur Fahrzeugführung eingesetzt (siehe [Fal+07; Kön+07; Kön09; Shi+10; Cho+12; Gao+12; Shi+12; Kat+13; Buß13|). Den Vorteilen bei der Regelungsgüte steht der hohe Rechenaufwand gegenüber.

\section{Wirksamkeit in Probandenstudien}

Mit prototypisch entwickelten Versuchsträgern und Fahrsimulatoren sind bereits einige Probandenstudien durchgeführt worden. In der Mehrzahl der Fälle konnte die Anzahl der Kollisionen gesenkt werden, wenn eine Ausweichassistenz eingeschaltet war (siehe [Del+10; Sou+11; Ito+13; Cho+14; Kel+14a; See+15]). In [Kat+14] konnte mit einer Lenkmomentenüberlagerung keine Verbesserung erzielt werden. In einer Studie mit einem Versuchsträger, beschrieben in [Sie+15], trat keine Senkung der Kollisionsrate auf, jedoch wurde mit Hilfe der Assistenz ein lateraler Sicherheitsabstand häufiger und früher erreicht. Durch das Testen verschiedener Begrenzungen des Torque Overlay konnte [Iwa+14] zeigen, dass sich das Fahrverhalten der Probanden verbessern lässt, je höher das Momentenlevel gewählt wird. Wird ein gewisses Level überschritten, nimmt die Verbesserung jedoch wieder $a b$, da der Fahrer anfängt, gegen die Überlagerung zu agieren. Eine wichtige Anforderung an ein Notausweichmanöver ist die stabile Fahrzeugführung. Hierzu testeten [Meh+10] und [Meh15] das Verhalten von Probanden nach dem Überfahren einer Kick Plate (bewegliche Bodenplatte, die das Fahrzeug zum Übersteuern bringt, sobald die Hinterachse die Platte überquert) mit und ohne einer Lenkempfehlung zur Stabilisierung des Fahrzeugs. Es konnte ebenfalls eine Verbesserung durch die Lenkmomentenüberlagerung erzielt werden. In [Rug+15] wird bereits der Effekt von Ausweichmanövern auf den entgegenkommenden Verkehr untersucht, um zu bestimmen, unter welchen Bedingungen ein Ausweichmanöver für den Gegenverkehr handhabbar ist.

\subsection{Inhalt und Ziel der Arbeit}

In der vorliegenden Arbeit werden vier verschiedene Verfahren zur Fahrzeugführung im Sinne der Kollisionsvermeidung in Notsituationen entwickelt, verglichen und bewertet. Die Verfahren heißen:

1. Bahnfolgeverfahren (Path Following Approach - PFA),

2. Online-Trajektorienoptimierungsverfahren (Online Trajectory Optimization - OTO),

3. Modellprädiktives Planungs- und Regelungsverfahren (Model Predictive Planning and Control - MPPC) und

4. Modellprädiktives Trajektorienscharverfahren (Model Predictive Trajectory Set Approach - MPTSA).

Das Ziel der Arbeit ist es, die unterschiedlichen Eigenschaften, Möglichkeiten, Herausforderungen und Grenzen der Verfahren aufzuzeigen, zu bewerten und miteinander $\mathrm{zu}$ vergleichen. 


\subsection{Gliederung}

In Kapitel 2 wird zunächst die durchgängige Entwicklungsmethode beschrieben, welche einen dreistufigen Prozess vorschlägt. Aufgrund der potentiellen Gefährdung durch das Notmanöver für Mensch und Maschine und der Möglichkeit reproduzierbare Überraschungsmomente bei Probandenstudien zu erzeugen, werden die häufig eingesetzten Entwicklungsschritte Simulation und Experiment im prototypischen Versuchsfahrzeug um eine Simulatorumgebung ergänzt. Der Aufbau, die Möglichkeiten und Gegebenheiten der drei Entwicklungsumgebungen werden beschrieben und die Ergebnisse der Probandenstudie im Simulator vorgestellt.

Kapitel 3 erläutert die in der Arbeit häufig verwendeten Prädiktionsmodelle der Fahrdynamik. Es werden die mit höherer Modellordnung steigenden Möglichkeiten diskutiert und gegen die höhere Rechenlast und Komplexität abgewogen.

Das Bahnfolgeverfahren, als einfachstes der vier Alternativen, wird in Kapitel 4 beschrieben und diskutiert. Nach der Beschreibung der Bahnplanung, der Quer- und Längsregelung werden Ergebnisse aus dem Fahrsimulator und dem Versuchsfahrzeug diskutiert.

Kapitel 5 stellt zunächst das aus der Robotik stammende Timed Elastic Band (TEB) vor. Im Anschluss wird ein zur Kollisionsvermeidung geeignetes Gütemaß vorgestellt sowie die Realisierung der Trajektorie mit unterlagerten Geschwindigkeits- und Kurswinkelreglern diskutiert. Das Kapitel schließt mit der Präsentation und Diskussion der Ergebnisse aus der Simulation.

In Kapitel 6 wird gezeigt, wie durch die Integration eines dynamischen Prädiktionsmodells ein simultanes Planungs- und Regelungsverfahren entsteht. Nach der Definition des Optimalsteuerungsproblems wird auch bei diesem Verfahren die Wahl des Gütemaßes diskutiert, welches aufgrund des Prädiktionsmodells im Vergleich zur OTO weitere Anteile enthält. Im Anschluss werden Simulationsergebnisse vorgestellt.

Kapitel 7] beschreibt das modellprädiktive Trajektorienscharverfahren zunächst grundlegend, bevor die adaptive Stellgrößendiskretisierung behandelt wird. Die Spezifika bei der Formulierung des Optimalsteuerungsproblems und die Wahl der Gütefunktion werden erläutert. Abschließend werden Ergebnisse aus der Simulation und dem Fahrsimulator untersucht.

In Kapitel 8 werden die vier Verfahren abschließend bewertend miteinander verglichen. Darüber hinaus werden weitere Anwendungsmöglichkeiten und Modifikationen diskutiert.

Das 9 . Kapitel fasst die Arbeit zusammen und gibt einen Ausblick auf weitere mögliche Untersuchungen.

Im Anhang A finden sich Definitionen und die Generalisierbarkeit des MPTSA wird in einem Exkurs beispielhaft behandelt. 


\section{2}

\section{Entwicklungsumgebungen}

\subsection{Durchgängige Entwicklungsmethode}

Bei der Vorausentwicklung mechatronischer Systeme wird häufig die modellbasierte Entwicklung angewendet. Für den Entwurf von Regelungssystemem wird dabei zunächst ein komplexes Streckenmodell erstellt, welches für viele Entwurfsverfahren zu einem regelungstechnischen Modell vereinfacht wird. Das Regelungssystem kann schnell und ohne Gefahr (mögliche Instabilität in der frühen Entwicklungsphase) in der Simulation erprobt werden, wodurch weitere Erkenntnisse über das Systemverhalten gewonnen werden und Analysen der Robustheit und Sensitivität gegenüber Parameteränderungen, verglichen mit dem Experiment, recht einfach durchgeführt werden können. Nach der Simulationsphase werden die Regelungssysteme in einem prototypisch aufgebauten Experimentalsystem erprobt. In der Fahrzeugsystemtechnik ist dies häufig das Versuchsfahrzeug. Bei einigen mechatronischen Systemen bieten sich Prüfstände an. Wird die Regelungsgüte als nicht ausreichend befunden, wird der Regler und/oder das Streckenmodell mit Hilfe der Simulation verbessert und erneut am Experimentalsystem erprobt. Auf diese Weise ergeben sich häufig mehrere Zyklen, bis ein zufriedenstellendes Ergebnis erreicht wird. Bei der vorliegenden Aufgabe der Entwicklung eines Notausweichassistenten ist das Experimentalsystem zwangsläufig ein Versuchsfahrzeug, da die Fahrdynamik geregelt werden muss und sich dies nicht in einem Prüfstand durchführen lässt. Die alleinige Erprobung des ESA im Versuchsfahrzeug ist mit den folgenden Nachteilen verbunden:

- Die Erprobung ist nur auf geschlossenen Teststrecken möglich, die eine ausreichende Freifläche und entsprechend lange Beschleunigungs- und Verzögerungsstreifen aufweisen. Der Transport des Versuchsträgers ist zeit- und kostenintensiv. Fahrbahnmarkierungen sind auf Fahrdynamikflächen häufig nicht vorhanden und müssen erst aufgebracht werden. Durch die Entwicklung der Notbremssysteme sind zwar realistische Hindernismodelle (Balloon Car) verfügbar, aber es ist schwierig bewegte Hindernisse mit vordefinierten Bewegungsprofilen auf einer Teststrecke zu realisieren. Entsprechende Anlagen sind bisher nicht verfügbar, sodass nur die Realisierung mit Testfahrern bliebe, was aufgrund der hohen Gefahr nicht vertretbar ist. Insbesondere bei komplexen Verkehrssituationen mit mehreren bewegten Hindernissen ist ein abgestimmter Verlauf schwierig und dessen Reproduzierbarkeit praktisch nicht gegeben. 
- Weitere Herausforderungen treten bei der Durchführung von Probandentests auf. Wenn z.B. das in der Motivation beschriebene Szenario (Übersehen des Stauendes) auf der Teststrecke nachgestellt werden soll, muss für den Überraschungseffekt ein Hindernis, vom Fahrer zunächst unbemerkt, plötzlich erscheinen bzw. auf die Fahrbahn geschoben werden oder der Fahrer muss abgelenkt werden. Beide Möglichkeiten werfen Probleme auf, insbesondere unter dem Gesichtspunkt der Reproduzierbarkeit. Darüber hinaus steht eine Vorrichtung, die ein Hindernis auf die Fahrbahn bewegen kann, der Forderung nach einer Freifläche entgegen.

Aus diesen Gründen ist es bei der Forschung und Entwicklung von Kollisionsvermeidungssystemen erforderlich, den Fahrsimulator als Zwischenstufe in den Entwicklungsprozess einzubringen. Dies ist zum einen zur Verlagerung der kostenintensiven Prüfgeländetests in die Simulatorumgebung und zum anderen zur Vermeidung der nur mit großen Aufwand durchführbaren Probandenstudien auf dem Testgelände vorteilhaft. Es sind eine Vielzahl von Fahrsimulatoren entwickelt worden, welche sich bezogen auf die vorliegende Arbeit wie folgt gruppieren lassen:

- Ein Tischsimulator besteht aus einem Gaming-Lenkrad mit Pedalerie, einem Bürostuhl und einem Computerbildschirm.

- Ein feststehender Fahrsimulator weist ein realistisches Mockup mit aktuiertem Lenkrad, Pedalerie, Fahrzeugsitz und ein aus mehreren Bildschirmen bestehendes Visualisierungssystem auf.

- Bei einem Fahrsimulator mit Bewegungssystem wird die Fahrzeugkabine durch ein Aktorsystem (z.B. Hexapod) aktuiert. Mit Hilfe von Projektoren wird eine $360^{\circ}$ Rundumsicht erreicht.

- Alternativ kann ein echtes Fahrzeug auf ein Bewegungssystem gestellt werden, was jedoch mit einem entsprechenden Umbau des Versuchsträgers verbunden ist.

Zunächst ist anzumerken, dass es auch Fahrsimulatoren gibt, die eine Mischung aus zwei oder mehreren Kategorien darstellen. Der Tischsimulator eignet sich bereits für einfache Untersuchungen, etwa zur Überprüfung von Szenarien oder als Vorstufe für einen aufwändigeren Simulator. Der feststehende Fahrsimulator eignet sich für viele Untersuchungen im Bereich der Forschung und Entwicklung von Fahrerassistenzsystemen. Insbesondere können mit dem Fahrsimulator Probandenstudien durchgeführt werden. Der Fahrsimulator mit Bewegungssystem ermöglicht die gleichen Untersuchungen wie der feststehende Fahrsimulator. Es entsteht jedoch ein noch realistischerer Eindruck auf Grund der Rückmeldung durch die Beschleunigungen an den Fahrer. Das Bewegungssystem ist jedoch mit einem höheren finanziellen und technischen Aufwand verbunden. Der Fahrsimulator mit einem echtem Versuchsfahrzeug erhöht die Detailtreue weiter. Da das gesamte Fahrzeug bewegt wird und damit der technische Aufwand sehr groß ist, muss abgewogen werden, ob gegenüber dem Fahrversuch wesentliche Vorteile bestehen. Erhalten bleibt die Eigenschaft des gefahrlosen Testens. Für die Untersuchungen und Ergebnisse in dieser Arbeit wird ein feststehender Simulator eingesetzt. Der Simulator erfüllt die Anforderungen, wenngleich ein Bewegungssystem 
im fahrphysikalischen Grenzbereich einen realistischeren Fahreindruck aufweisen kann. Ein Bewegungssystem stand jedoch nicht zur Verfügung. Der verwendete Fahrsimulator bietet die folgenden Möglichkeiten:

- Es können Szenarien mit vorgegebenen Straßenverläufen und Bewegungsprofilen anderer Verkehrsteilnehmer definiert werden.

- Andere Verkehrsteilnehmer können (für den Fahrer) überraschend platziert werden.

- Durch die Verwendung einer Rapid-Prototyping-Hardware des Unternehmens dSPACE und des CAN BUS zur Kommunikation der Komponenten wird die gleiche Toolkette verwendet wie im Versuchsfahrzeug. Hierdurch lassen sich Algorithmen einfach und schnell in den Prototypen übertragen und testen.

- Versuche und Probandenstudien können ohne Gefahr für Mensch und Maschine durchgeführt werden.

- Bei Probandenstudien lässt sich eine hohe Reproduzierbarkeit erreichen.

Die Grenzen des Simulators werden bei der Durchführung von Probandentests sichtbar:

- Probanden haben trotz der bedrohlichen Notsituation keine Angst vor den Folgen einer Kollision.

- Auf Grund der fehlenden Beschleunigungen entspricht zum einen der Fahreindruck nicht exakt der Realität und zum anderen kann bei ungeübten Probanden die Simulatorkrankheit auftreten. Die großen Bildschirme und das detailgetreue Mockup erzeugen jedoch einen ausreichend realistischen Eindruck.

Die Ergebnisse von Probandenstudien sollten daher in ihrer Quantität nicht überbewertet werden, sind aber aus Sicht des Autors qualitativ aussagekräftig. Bei der Bewertung des feststehenden Simulators ist zu bedenken, dass Probandenstudien auf Teststrecken ebenfalls nur eingeschränkt gültig sind, da aus Gründen der Sicherheit keine massiven Hindernisse verwendet werden können und so die Angst vor einer Kollision ebenfalls nicht der realen Situation entspricht.

\subsection{Fahrsimulator}

\section{Aufbau}

Der Fahrsimulator besteht aus einem Mockup der Firma Foerst Fahrsimulatoren GmbH, einer Fahrsimulationssoftware „SILAB“ der Firma WIVW (Würzburger Institut für Verkehrswissenschaften $\mathrm{GmbH}$ ), drei großen Monitoren zur Visualisierung und einer Rapid Prototyping Hardware. Der Fahrsimulator ist in Abbildung 2.1 zu sehen. Das Mockup besteht aus der Pedalerie, einem aktuierten Lenkrad, einem Schalthebel, einer Handbremse, einem Sicherheitsgurt und mehreren Schaltern (z.B. Lenkstockhebel). Die Fahrsimulationssoftware SILAB wird auf insgesamt sechs Rechnern ausgeführt. Hierin integriert sind die Visualierung, die Verkehrssimulation, das Fahrdynamikmodell und die Kommunikation über den CAN BUS. Die Rapid Prototyping Hardware erlaubt es MATLAB/Simulink Modelle zu kompilieren und in Echtzeit zu betreiben. 


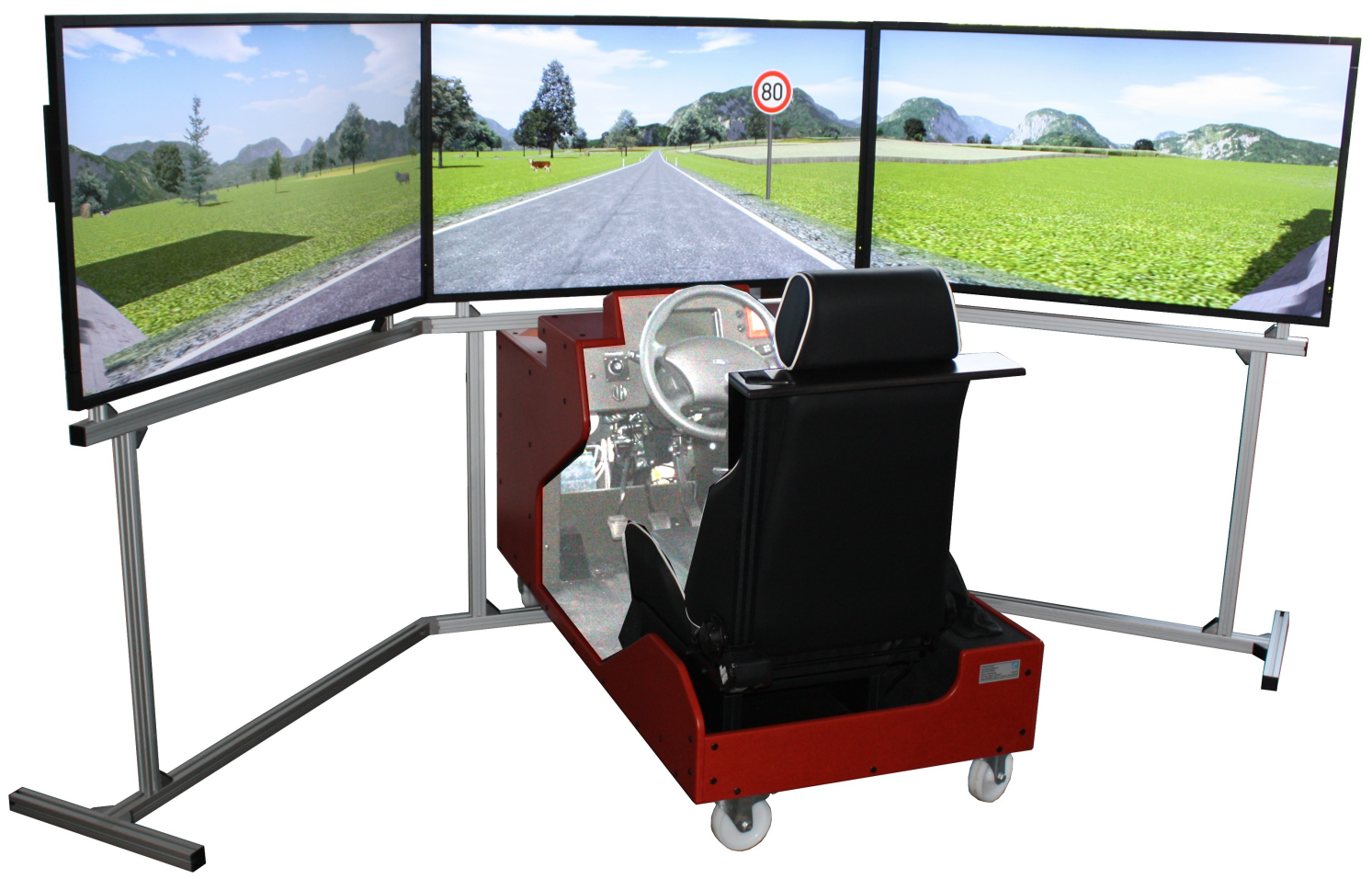

Abbildung 2.1.: Bild des feststehenden Fahrsimulators.

\section{Modellbildung und Regelung des Lenkaktuators}

Das Lenkrad des Mockups wird mit einem BLDC-Motor aktuiert. Das Stellsignal wird von einem Microcontroller getrieben, welcher das entsprechende Signal vom CAN BUS liest. Die einzelnen nichtlinearen Übertragungsglieder des Blockschaltbildes der offenen Kette sind in Abbildung 2.2 dargestellt. Zur Modellierung wird die vereinfachende

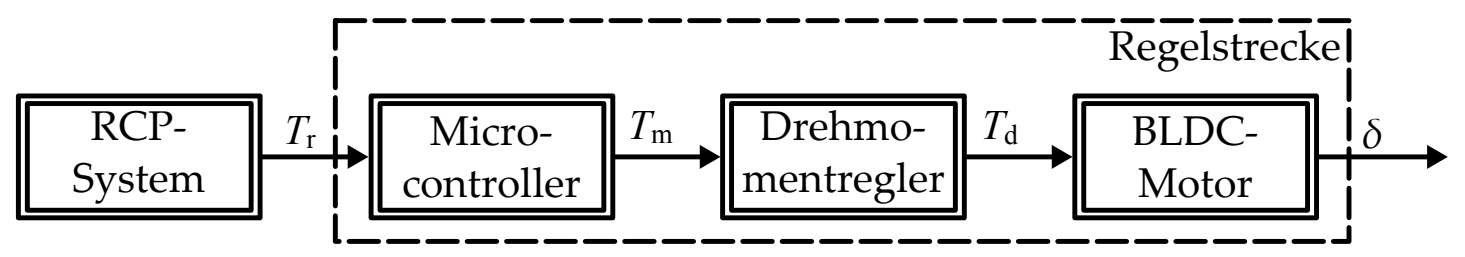

Abbildung 2.2.: Offener Kreis des Lenksystems.

Annahme getroffen, dass sich der Drehmomentregler wie ein lineares System erster Ordnung und der BLDC-Motor (inklusive Lenksäule und Lenkrad) wie ein lineares System zweiter Ordnung verhält. Da das Lenksystem trägheitsbehaftet ist, scheidet ein System erster Ordnung aus. Die obige Annahme stellt somit das einfachste Modell dar, mit dem das Verhalten beschrieben werden kann (ohne Berücksichtigung von zusätzlichen Modellreduktionen). Im Folgenden werden die Parameter der Regelstrecke identifiziert. Die Totzeit durch die Informationsverarbeitung wird nicht explizit im Modell berücksichtigt. Das Modell hat die Übertragungsfunktion

$$
G_{\mathrm{LS}}(s)=\frac{k_{\mathrm{LS}}}{\left(T_{\mathrm{TC}} s+1\right)\left(J_{\mathrm{LA}} s^{2}+b_{\mathrm{LA}} s+1\right)}
$$


Basierend auf dem Modell wird ein Zustandsregler nach Riccati entworfen. Da nur eine Zustandsgröße messtechnisch erfasst wird, müssen die anderen beiden durch einen Beobachter rekonstruiert werden. Ein Vorteil des Zustandsreglers mit Beobachter ist, dass die Lenkrate rekonstruiert wird, welche auch für die überlagerten Regler (modellprädiktive Verfahren in den Kapiteln 6und 7) zur Verfügung steht. Die Reglerauslegung erfolgt durch manuelles Optimieren der Diagonalmatritzen $\mathbf{Q}_{\mathrm{LS}}$ und $\mathbf{S}_{\mathrm{LS}}$ des Gütemaßes

$$
J_{\mathrm{LS}}=\int_{0}^{\infty}\left[\mathbf{x}_{\mathrm{LS}}{ }^{T} \mathbf{Q}_{\mathrm{LS}} \mathbf{x}_{\mathrm{LS}}+\mathbf{u}_{\mathrm{LS}}{ }^{T} \mathbf{S}_{\mathrm{LS}} \mathbf{u}_{\mathrm{LS}}\right] \mathrm{d} t .
$$

Das Führungsfilter wird auf stationäre Genauigkeit ausgelegt. Aufgrund der integralen Eigenschaft der Strecke ist dies auch bei dem vergleichsweise einfachen Modell ausreichend. In Abbildung 2.3 ist die Sprungantwort des auf diese Weise gewonnenen Regelkreises dargestellt. Die Anstiegszeit ist gering, aber es tritt Überschwingen auf. Je nach Anwendungszweck (Notmanöver oder normale Fahrt) werden unterschiedliche Anforderungen an die Dynamik des geschlossenen Lenkwinkelregelkreises gestellt. Die hier gewählte Sprungantwort hat sich für Notmanöver als geeignet erwiesen, da die geringe Anstiegszeit hochdynamische Manöver ermöglicht. Durch die geeignete Wahl des Gütemaßes lässt sich der Kompromiss zwischen Anregelzeit und Überschwingweite einstellen. Auf Grund der nicht modellierten Reibungseffekte bleiben

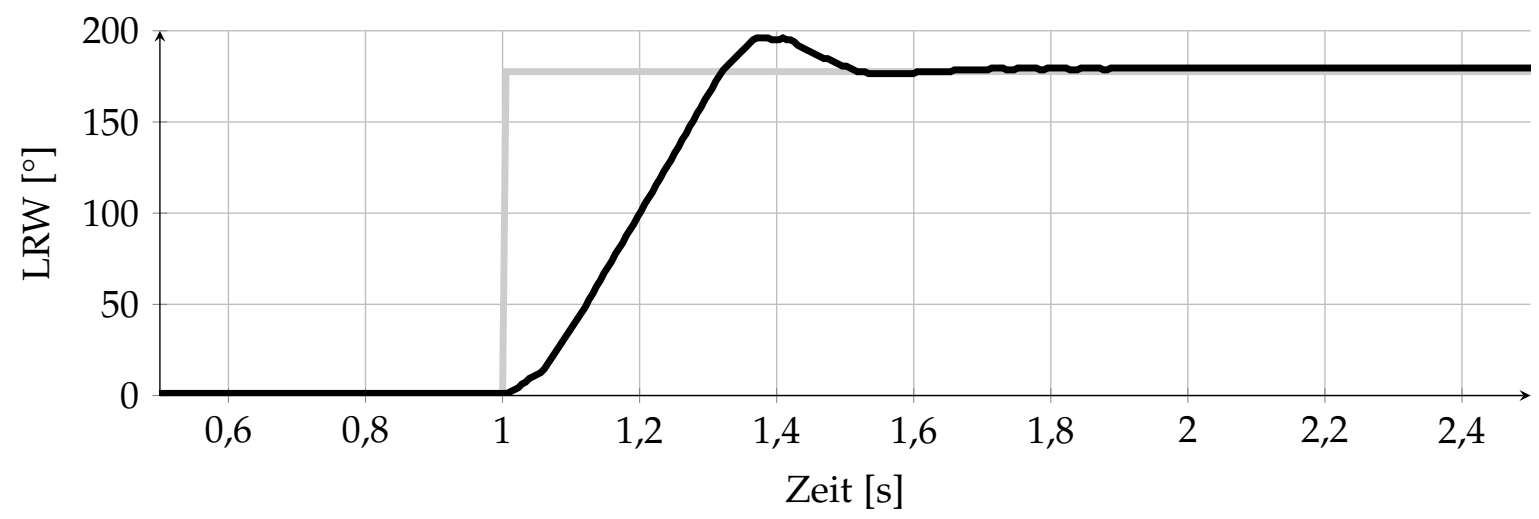

Abbildung 2.3.: Sprungantwort des geschlossenen Lenkradwinkelregelkreises. Die Sollgröße ist in grau und die Istgröße in schwarz dargestellt.

kleine stationäre Abweichungen, welche aber für das Notausweichmanöver keine große Bedeutung haben.

\subsection{Anwendung des Fahrsimulators in der Probandenstudie}

Um aufzuzeigen, dass sich der Fahrer durch Lenkmomentenüberlagerung in Notsituationen korrigieren lässt, wurde eine Probandenstudie im Fahrsimulator durchgeführt. Hierbei wurde der in [Kel+14a] beschriebene Algorithmus (Bahnfolgeverfahren) verwendet. Die Probanden wurden gebeten, das Fahrzeug durch Lenkeingaben auf der rechten Fahrspur einer Autobahn zu halten. Die Geschwindigkeit wurde durch einen Tempomaten auf $100 \mathrm{~km} / \mathrm{h}$ eingeregelt. Dann wurde den Probanden unerwartet ein stehender LKW vor das Ego-Fahrzeug platziert. Durch eine Leitplanke unmittelbar 
neben der rechten Fahrbahn bot der Standstreifen keine alternative Ausweichmöglichkeit. Die Probanden wurden darüber informiert, dass diese Situation eintreten wird, jedoch nicht genau zu welchem Zeitpunkt. Zusätzlich wurde die Vorgabe gemacht, dass die Kollision durch ein Notausweichmanöver zu verhindern sei und die Bremse nicht eingesetzt werden soll. Jeder Proband absolvierte zehn Versuche, wobei das Hindernis an unterschiedlichen Stellen platziert wurde. Der Proband war dadurch zwar aufmerksam, konnte sich aber nicht in dem Maße auf das Manöver vorbereiten wie es bei der Anfahrt auf ein sichtbares Ziel möglich wäre. Bei der Hälfte der Versuche war der ESA eingeschaltet und löste automatisch aus, sobald die maximale Querbeschleunigung entlang der geplanten Bahn das Beschleunigungspotential erreicht hatte (siehe Definition A.1.14). Die Probanden wurden nicht darüber informiert, ob das System eingeschaltet war oder nicht, sodass sie stets bestrebt waren die Kollision selbst $\mathrm{zu}$ vermeiden. In einer ersten Untersuchung mit vier Probanden wurde der LKW $40 \mathrm{~m}$ (entspricht TTC = 1,44 s) vor dem Ego-Fahrzeug platziert. In Tabelle 2.1 sind die Ergebnisse dargestellt. Es ist ersichtlich, dass die Anzahl der Kollisionen reduziert

Tabelle 2.1.: Ergebnisse der ersten Probandenstudie.

\begin{tabular}{|c|c|c|}
\hline & mit Kollision & ohne Kollision \\
\hline ESA ein & 5 bzw. 25\% & 15 bzw. $75 \%$ \\
\hline ESA aus & 14 bzw. 70\% & 6 bzw. 30\% \\
\hline
\end{tabular}

wird, wenn der ESA eingeschaltet ist. Die Situation wird zwar den Notsituationen zugeordnet, jedoch waren die Probanden in 30\% der Versuche in der Lage, das Manöver erfolgreich durchzuführen. Aus diesem Grund wurde für sechs weitere Probanden die Distanz zum LKW auf $35 \mathrm{~m}$ (entspricht $T T C=1,26 \mathrm{~s}$ ) reduziert. In Tabelle 2.2 sind die Ergebnisse dargestellt. Während der Prozentsatz der kollisionsfreien Manöver

Tabelle 2.2.: Ergebnisse der zweiten Probandenstudie.

\begin{tabular}{|c|c|c|}
\hline & mit Kollision & ohne Kollision \\
\hline ESA ein & 8 bzw. 26,7 \% & 22 bzw. 73,3 \% \\
\hline ESA aus & 26 bzw. 86,7 \% & 4 bzw. 13,3 \% \\
\hline
\end{tabular}

auf $13,3 \%$ sinkt, wenn das System ausgeschaltet ist, bleibt der prozentuale Anteil bei eingeschalteter Assistenz mit 73,3\% auf dem gleichen hohen Niveau wie in der ersten Probandenstudie. In der Abbildung 2.4 sind Messungen zweier charakteristischer Fahrertypen zu erkennen. In Abbildung 2.4(a) ist das Verhalten des zu vorsichtigen Fahrers zu erkennen. Dieser Fahrertyp traut sich nicht, die großen Lenkradwinkel zu stellen, die für das Manöver nötig sind. Als Folge kollidiert er direkt zu Beginn mit dem Hindernis. Durch Eingriff des ESA konnte der Fahrer in einem anderen Versuch die Kollision vermeiden, indem der Lenkradwinkel vor allem zu Beginn des Manövers größer gewählt wurde. Dies ist in Abbildung 2.4(b) zu erkennen. 

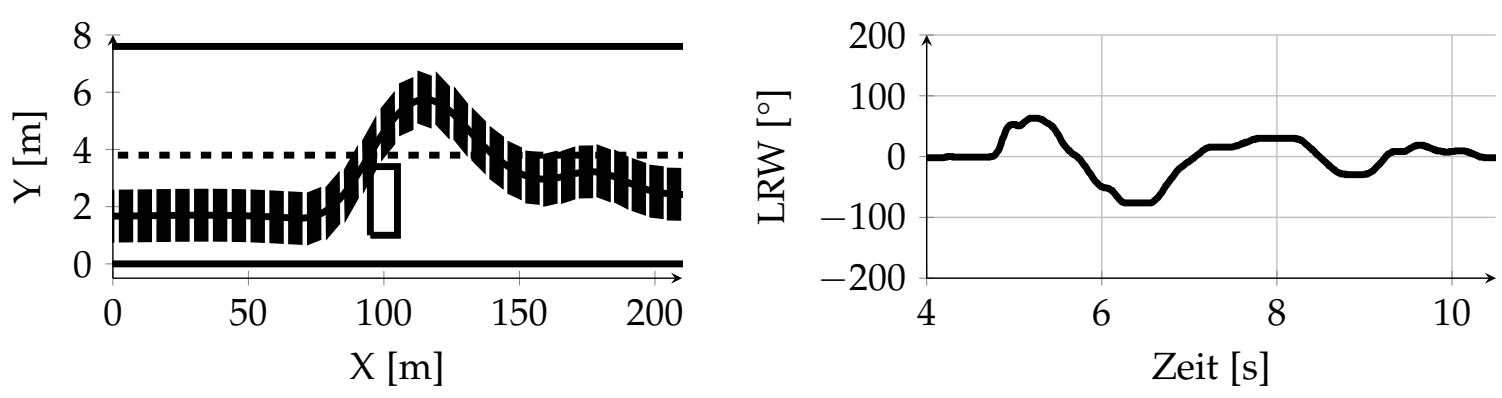

(a) Messung des zu vorsichtigen Fahrertyps ohne ESA.
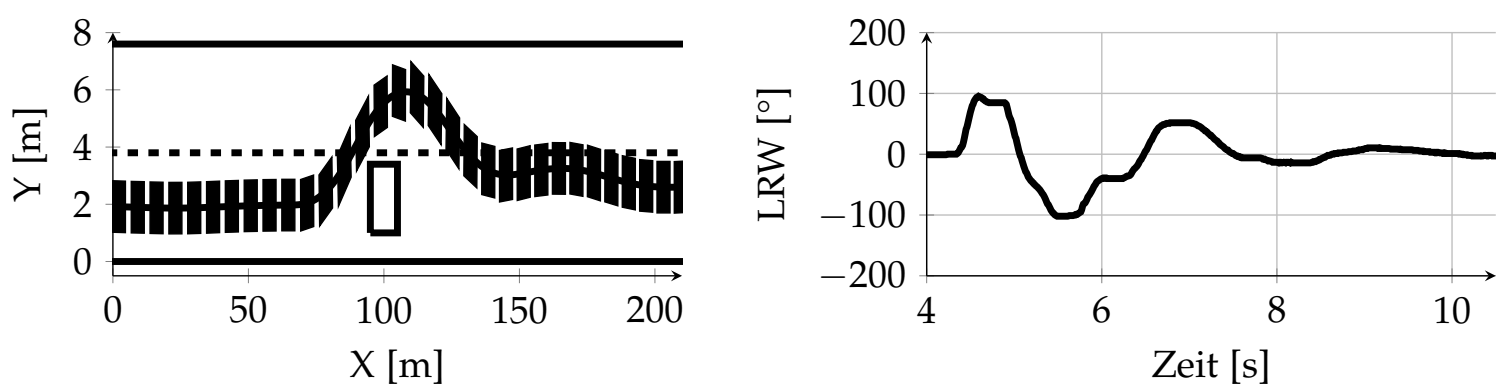

(b) Messung des zu vorsichtigen Fahrertyps mit ESA.
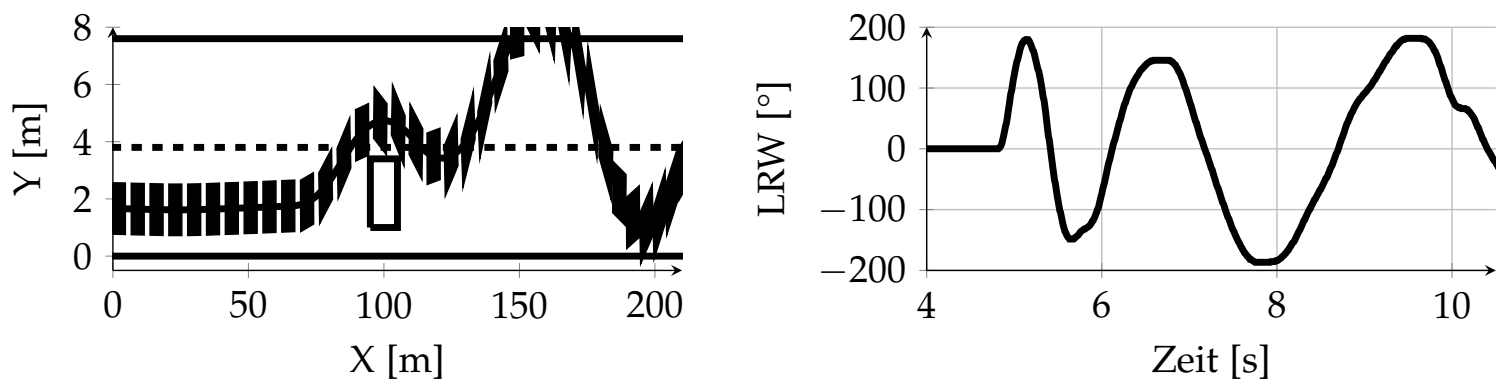

(c) Messung des unvorsichtigen Fahrertyps ohne ESA.
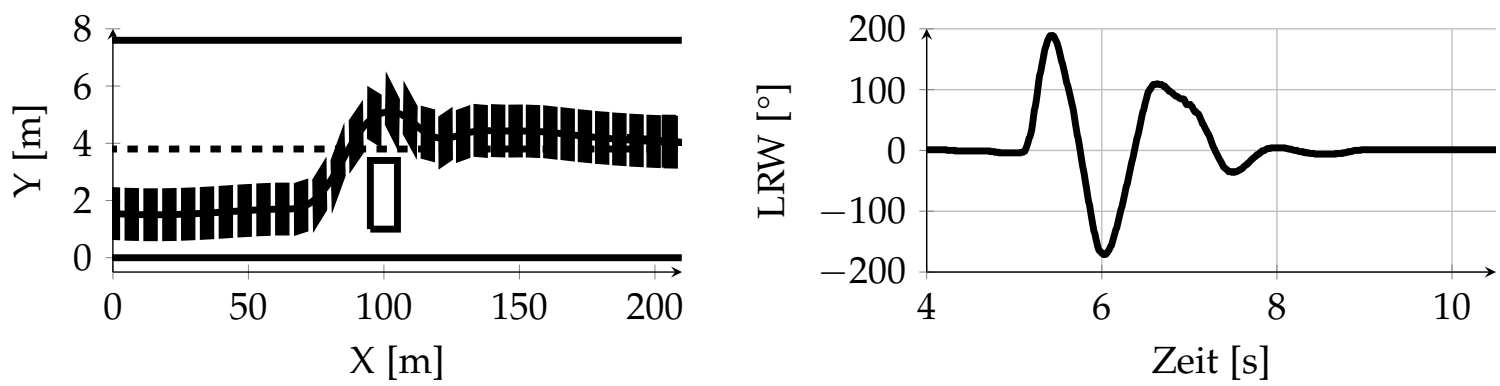

(d) Messung des unvorsichtigen Fahrertyps mit ESA.

Abbildung 2.4.: Messungen zweier charakteristischer Fahrertypen. Das Ego-Fahrzeug ist in Abständen von $0,25 \mathrm{~s}$ als ausgefülltes Viereck dargestellt.

Das Verhalten eines anderen Fahrertyps ist in Abbildung 2.4(c) dargestellt. Der Fahrer vermeidet die Kollision im ersten Augenblick. Auf Grund der großen Lenkbewegungen destabilisiert er das Fahrzeug. Es sind die typischen Schleuderbewegungen zu erkennen, die in Kollisionen mit der Straßenbegrenzung enden. Der Fahrer versucht das Fahrzeug durch Gegenlenken zu stabilisieren. Er verhindert zwar eine Drehung 
des Fahrzeugs, bewirkt jedoch abwechselnde Übersteuersituationen nach links und rechts. Dies liegt an den zu groß gewählten Lenkradwinkeln während des Gegenlenkens. Auch dieser Fahrertyp konnte bei einem anderen Versuch durch das System korrigiert werden, sodass ein stabiles und kollisionsfreies Manöver erreicht wird, wie in Abbildung 2.4(d) zu sehen ist. Insbesondere beim Gegenlenken (zwischen $t=6,5 \mathrm{~s}$ und $t=7,5 \mathrm{~s}$ ) wird der maximale Lenkradwinkel auf ein ausreichendes Maß reduziert. Insgesamt zeigen die Ergebnisse die Wirksamkeit der Lenkmomentenüberlagerung in dieser Probandenstudie. Es ist daher zu erwarten, dass auch im realen Straßenverkehr eine Verbesserung des Kollisionsvermeidungsverhaltens der Fahrer erreicht wird.

\subsection{Prototypisches Versuchsfahrzeug}

\section{Aufbau}

Als Versuchsfahrzeuge werden Opel Insignia eingesetzt (siehe Abbildung 2.5). Die

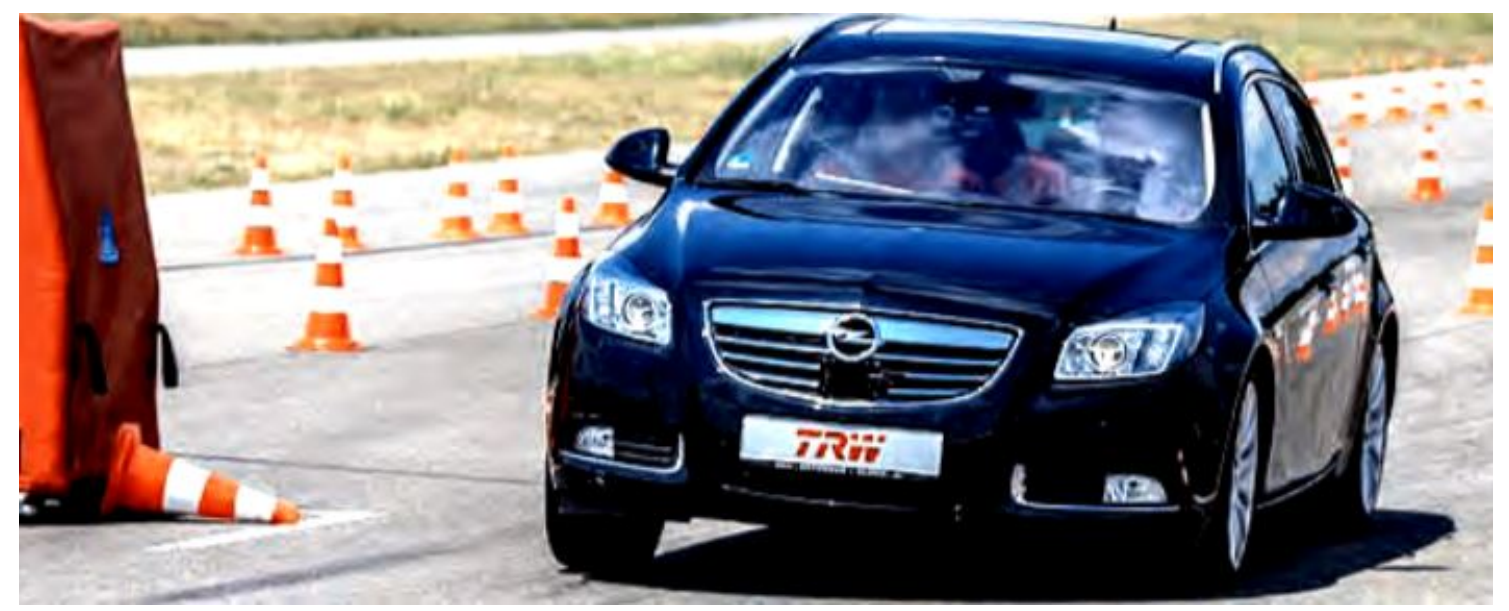

Abbildung 2.5.: Bild des prototypischen Versuchsfahrzeugs während eines Notausweichmanövers (Quelle: TRW Automotive $\mathrm{GmbH}$ ).

Fahrzeuge sind mit einer elektromechanischen Servolenkung, welche zur Überlagerung zusätzlicher Lenkmomente verwendet wird, ausgerüstet. Die heute üblichen Bremsschlupfregelungssysteme, welche die Funktionen ABS und ESC realisieren, sind ebenfalls verbaut. Eine Schnittstelle zum Aufbringen zusätzlicher Bremsmomente im Sinne einer Notbremsung bzw. zur Durchführung von kombinierten Brems- und Lenkmanövern im Sinne des Notausweichens war nicht gegeben. Zur Erfassung des Umfeldes wurden nach vorne gerichtete Sensoren in Form einer Kamera und eines Radars verbaut, sodass Hindernisse und Spurmarkierungen erkannt werden können. Es besteht zudem die Möglichkeit, einen mechatronischen Gurtstraffer zu aktivieren und den Fahrer durch audiovisuelle Hinweise auf eine kritische Verkehrssituation hinzuweisen. In Abbildung 2.6 ist eine vereinfachte Darstellung des CAN BUS mit den für die ESA Funktion wichtigen Steuergeräten dargestellt. Das RCP-System liest die fahrdynamischen Größen, welche vom Lenkungs- und Bremsensteuergerät gesendet werden, und die Umfeldinformationen, welche vom Radar und der Kamera zur Verfügung gestellt werden. Die Lenkung, der mechatronische Gurtstraffer (ACR - Active 


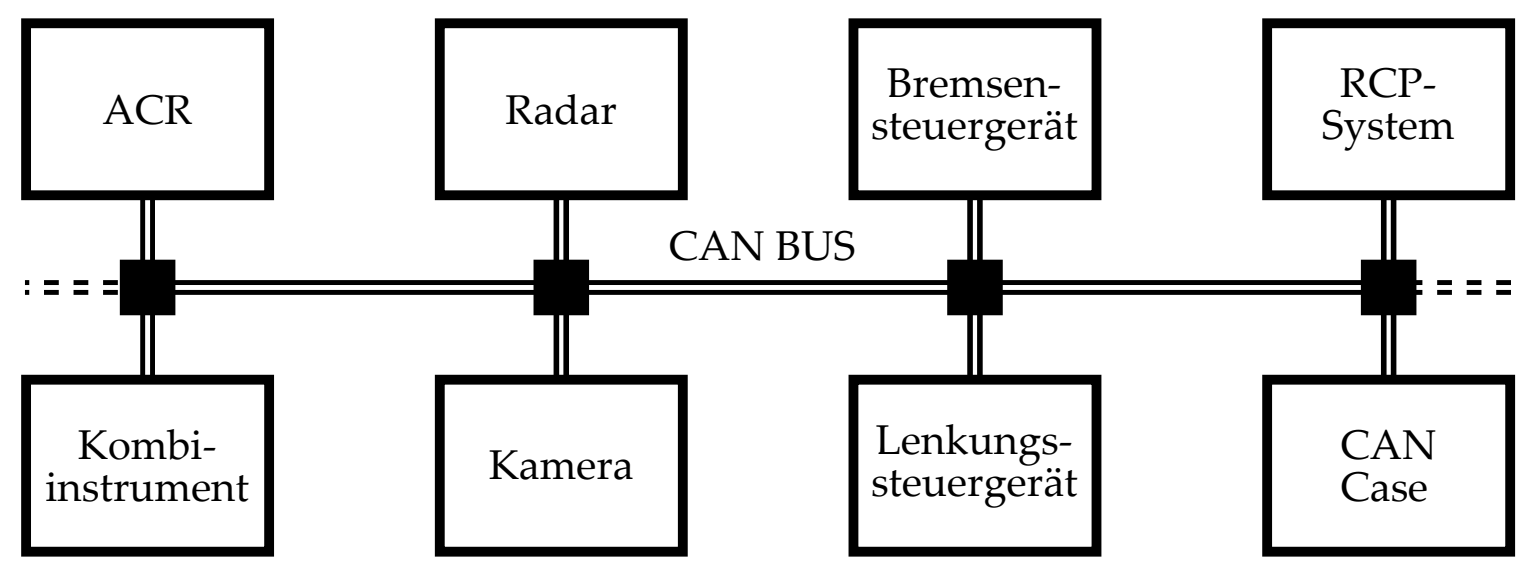

Abbildung 2.6.: Vereinfachte Darstellung des CAN BUS im Versuchsfahrzeug.

Control Retractor) und das Kombiinstrument empfangen die Stell- bzw. Informationssignale vom RCP-System. Mit Hilfe des CAN Case werden alle relevanten Signale aufgezeichnet.

\section{Elektrisches Lenksystem und Drehmomentschnittstelle}

In modernen Kraftfahrzeugen werden vermehrt elektromechanische Servolenkungen eingesetzt. Zum einen werden so der Kraftstoffverbrauch und die Anzahl der hydraulischen Komponenten des Fahrzeugs gesenkt und zum anderen kann durch entsprechende Software das Lenkgefühl einfacher und genauer eingestellt werden als mit hydraulischer Lenkkraftverstärkung. Darüber hinaus lassen sich neben der Servounterstützung weitere Funktionen realisieren. Hierzu zählen viele Fahrerassistenzsysteme wie der Einparkassistent oder der Spurhalteassistent. Der ESA benötigt ebenso ein elektromechanisches Lenksystem, um den Fahrer die richtige Lenkweise vorzuschlagen oder den Lenkwinkel im geschlossenen Regelkreis zu manipulieren. Das

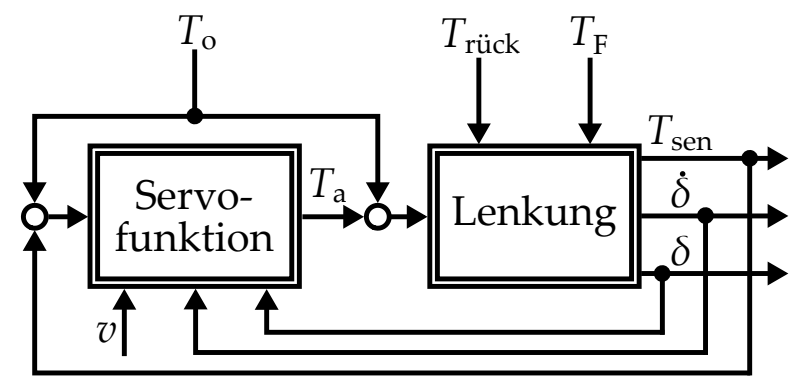

(a) Blockschaltbild der Dual Torque Overlay Schnittstelle.

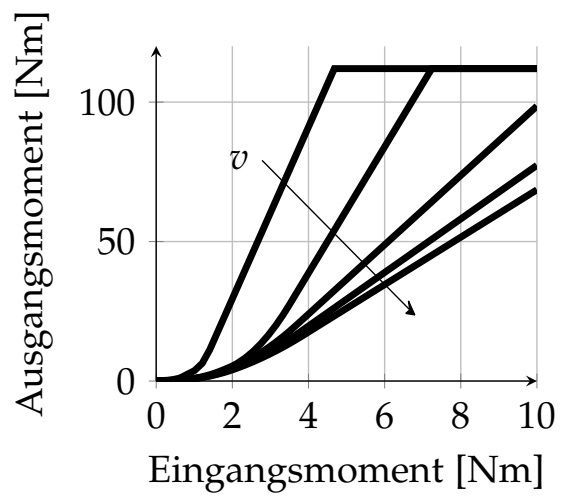

(b) Verstärkungskennlinien (Boost Curves) für mehrere Geschwindigkeiten.

Abbildung 2.7.: Eigenschaften der Lenkungsschnittstelle. 
Überlagerungsmoment wird über die sogenannte Dual Torque Overlay Schnittstelle übermittelt. Dies bedeutet, dass das zusätzliche Drehmoment sowohl vor (Input Torque Overlay) als auch hinter (Output Torque Overlay) dem Block zur Berechnung der Servofunktion softwaretechnisch addiert wird. Dies ist in Abbildung 2.7(a) dargestellt. Die Servofunktion setzt sich aus den nichtlinearen Steuerungs- und Regelungsanteilen "Boost Curve", "Active Return" und weiteren dämpfenden Funktionen zusammen. Von den genannten Unterfunktionen ist die „Boost Curve“ die Grundfunktion und hat dementsprechend den größten Einfluss auf das gestellte Unterstützungsmoment. In Abbildung 2.7(b) sind die Verstärkungskennlinien für verschiedene Geschwindigkeiten dargestellt. Es ist ersichtlich, dass sich kleine Überlagerungsmomente in erster Linie durch das Output Torque Overlay und große Momente durch das Input Torque Overlay bemerkbar machen.

\section{Lenkradwinkelregelkreis}

In Abbildung 2.8 ist das Blockschaltbild des Lenkradwinkelreglers dargestellt. Der Regler besteht aus einer nichtlinearen statischen Vorsteuerung, einem PD-Regler und einem Dämpfungsanteil für hohe Lenkraten. Die Stellgröße wird auf $6 \mathrm{Nm}$ begrenzt, sodass der Fahrer den Regler übersteuern kann. Alle Steuer- und Regleranteile sind

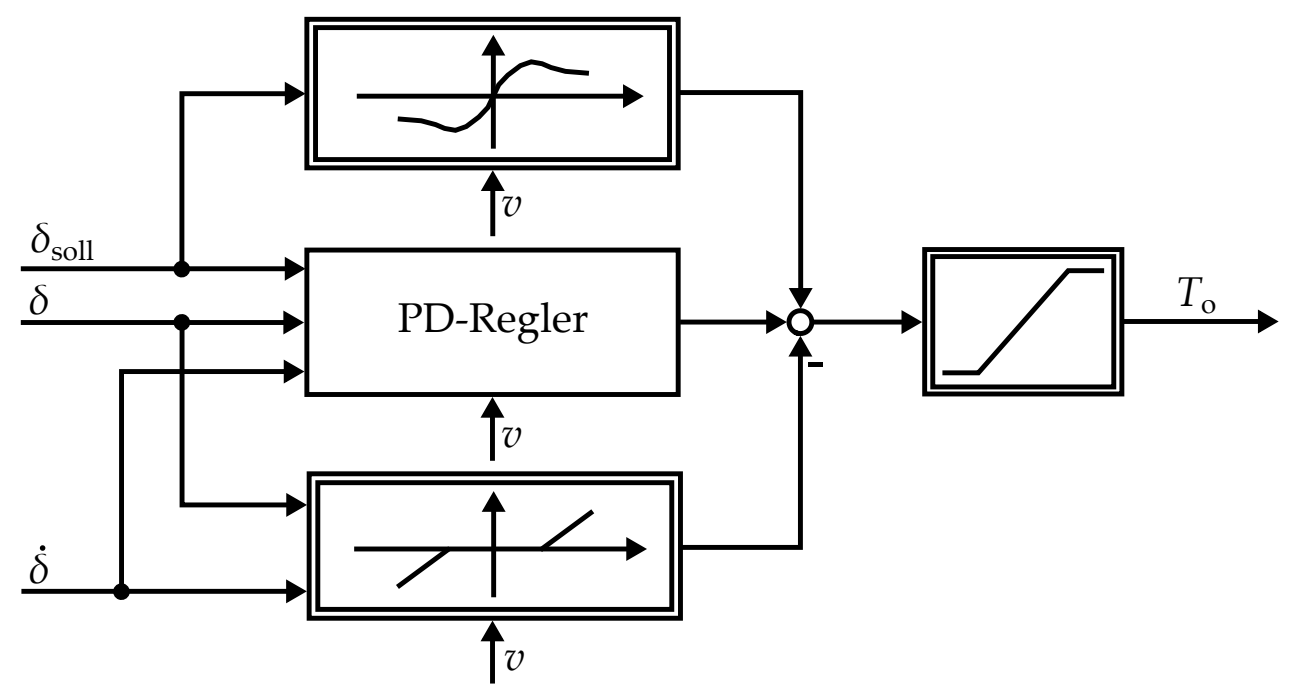

Abbildung 2.8.: Blockschaltbild des Lenkradwinkelreglers.

geschwindigkeitsadaptiv durch gesteuerte Adaption. Die zur Dämpfung hoher Lenkraten verwendete Kennlinie ist zusätzlich abhängig vom Lenkradwinkel. Dies ist darin begründet, dass beim Zuziehen der Lenkung die Rückstellkräfte gegen das Überlagerungsmoment, beim Öffnen hingegen gleichsinnig gerichtet sind. Ohne zusätzliche Dämpfung ergibt sich ein stark schwingendes Verhalten beim Einregeln der Geradeausstellung, wenn zuvor (betragsmäßig) große Lenkradwinkel gestellt worden sind. Die statische Kennlinie der nichtlinearen Vorsteuerung wird experimentell bestimmt. Hierzu werden konstante Überlagerungsmomente auf die Lenkung gegeben und die stationären Lenkwinkel gemessen. In Abbildung 2.9 ist eine Sprungantwort dargestellt. Verglichen mit Abbildung 2.3 fällt auf, dass der Lenkradwinkelregler im Fahrzeug den stationären Endwert nicht erreicht. Dies ließe sich durch einen I-Anteil bewerkstelligen. 


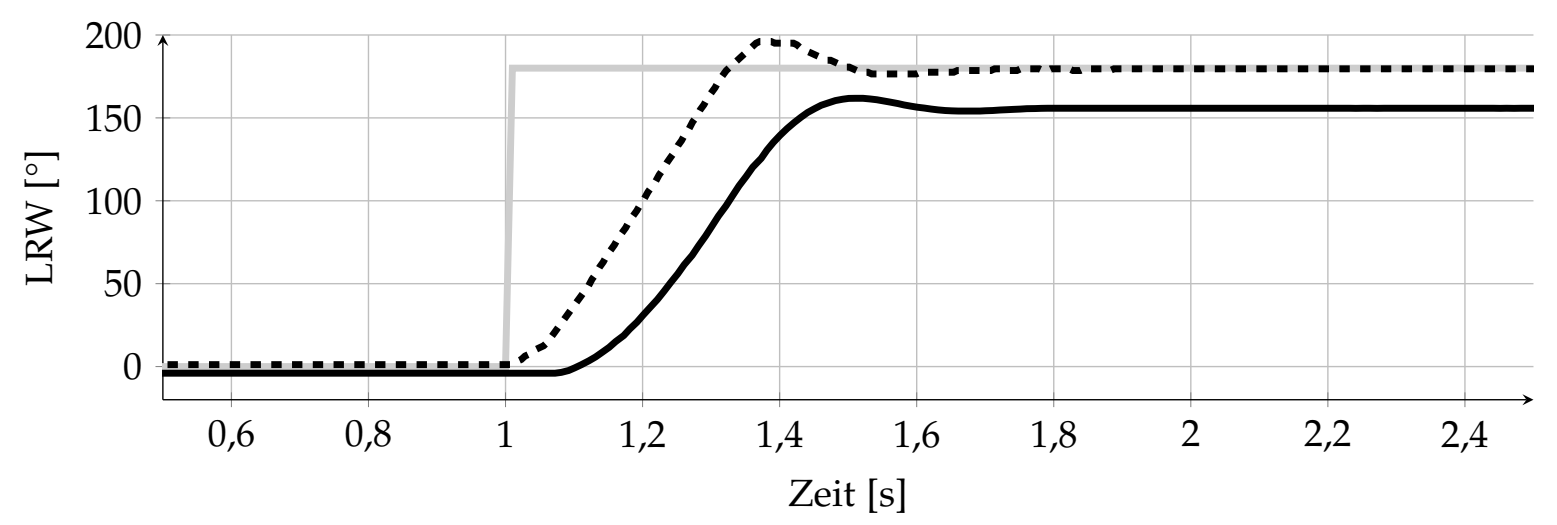

Abbildung 2.9.: Sprungantwort des Lenkradwinkelregelkreises im Versuchsfahrzeug in schwarz und durchgezogen. Zum Vergleich ist der Verlauf des Lenkradwinkelregelkreises des Fahrsimulators schwarz und gestrichelt dargestellt. Die Sollgröße ist in grau dargestellt.

Der Regler kommt jedoch als unterlagerter Regler zum Einsatz. Die Anforderung liegt daher nicht auf der stationären Genauigkeit sondern vielmehr auf einer schnellen Anstiegszeit bei mäßigem Überschwingen. Der Vergleich mit dem Lenkradwinkelregler im Fahrsimulator zeigt, dass neben den stationären Abweichungen im Wesentlichen die Totzeiten verschieden sind. Davon abgesehen ist das dynamische Übergangsverhalten vergleichbar.

\section{Fahrerinformationssystem und mechatronischer Gurtstraffer}

Fährt das Ego-Fahrzeug auf ein Hindernis zu, so steigt die Kritikalität zunächst langsam und dann immer schneller an. Ab einem gewissen Punkt erkennt das Radarsteuergerät, dass eine kritische Situation vorliegt und klassifiziert das Objekt als "Collision Warning Track". Sobald dies geschieht, wird der Fahrer akustisch (durch einen Warnton) und visuell (durch eine Anzeige im Kombiinstrument) vor der bevorstehenden Kollision gewarnt. Reagiert er nicht und überschreitet ein Kritikalitätsmaß einen ersten Grenzwert, so wird der reversible mechatronische Gurtstraffer ausgelöst. Steigt nach der Spannung der Gurte die Kritikalität weiter an und überschreitet einen zweiten Grenzwert, so wird im Falle eines autonomen Systems das Ausweichmanöver eingeleitet. Bei einem assistierenden System muss auf die initiale Lenkbewegung des Fahrers gewartet werden. Durch den Gurtstraffer werden die Insassen besser in den Sitzen fixiert und Bewegungen des Kopfes und Oberkörpers bei dem folgenden Ausweichmanöver werden minimiert. Die Fixierung verhindert zuverlässig, dass der Kopf der Insassen mit der Fahrzeugstruktur (z.B. der Seitenscheibe) kollidiert. In [Haß05] wird gezeigt, wie auch beim Fahrzeugüberschlag das Risiko von Kopfverletzungen mit einem Gurtstraffer reduziert werden kann. Da der Fahrer nicht immer durch das Assistenzsystem korrigiert werden kann (siehe Abschnitt 2.3) und Kollisionen mit anderen Verkehrsteilnehmern potentiell möglich sind, werden die Verletzungen durch einen vorgespannten Gurt reduziert. 


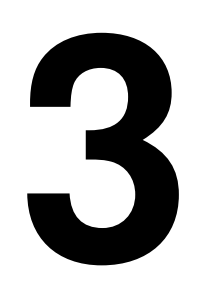

\section{Prädiktionsmodelle}

In diesem Kapitel wird zunächst die Beschreibung der Fahrkinematik mit einem Massenpunktmodell vorgestellt. Anschließend werden mögliche Fahrdynamikmodelle angegeben, welche sich zur Anwendung für modellprädiktive Verfahren eignen. Hierin ist ein Reifenmodell für kombinierten Quer- und Längsschlupf integriert. Danach werden Modelle der Aktuatorregelkreise (Lenkradwinkelregelkreis und Schlupfregelkreis) aufgestellt. Die Modelle werden hinsichtlich der Aktuatorkonfiguration und der damit verbundenen Komplexität bei der Anwendung als Modell zur prädiktiven Regelung verglichen und bewertet. Abschließend wird das Gesamtmodell ausgewählt.

\subsection{Massenpunktmodell}

Das Massenpunktmodell ist ein Modell zur Beschreibung von kinematischen Größen entlang von Bahnen bzw. Trajektorien. Der Massenpunkt hat bei ebener Bewegung zwei Freiheitsgrade $(X, Y)$. Der Schwimmwinkel kann nicht beschrieben werden, da ein Orientierungsfreiheitsgrad fehlt. Es ist somit nicht möglich, die Stabilität zu untersuchen, zumal es sich um ein statisches System handelt. Es können jedoch kinematische Größen entlang der Trajektorie berechnet werden. Die Minimierung und/oder Beschränkung dieser Größen auf Grenzwerte bewirken Trajektorien, welche die fahrdynamischen Reaktionen gering halten. Die kinematischen Größen entlang einer diskretisierten Trajektorie $\left(X_{k}, Y_{k}\right)$ lassen sich in natürlichen Koordinaten $\left(\mathbf{e}_{\mathrm{t}}, \mathbf{e}_{\mathrm{n}}\right)$ (siehe Abbildung 3.1(a)) wie folgt angeben:

$$
\begin{gathered}
\lambda_{k}=\arctan \frac{Y_{k}-Y_{k-1}}{X_{k}-X_{k-1}}, \dot{\lambda}_{k}=\frac{\lambda_{k}-\lambda_{k-1}}{\Delta T}, \\
a_{\mathrm{n}, k}=v_{k} \dot{\lambda}_{k}, j_{\mathrm{n}, k}=\frac{a_{\mathrm{n}, k}-a_{\mathrm{n}, k-1}}{\Delta T}+a_{\mathrm{t}, k} \dot{\lambda}_{k}, \\
v_{k}=\frac{\sqrt{\left(X_{k}-X_{k-1}\right)^{2}+\left(Y_{k}-Y_{k-1}\right)^{2}}}{\Delta T}, \\
a_{t, k}=\frac{v_{k}-v_{k-1}}{\Delta T}, j_{\mathrm{t}, k}=\frac{a_{\mathrm{t}, k}-a_{\mathrm{t}, k-1}}{\Delta T}-a_{\mathrm{n}, k} \dot{\lambda}_{k} \\
\text { und } a_{\mathrm{tot}, k}=\sqrt{a_{\mathrm{t}, k}^{2}+a_{\mathrm{n}, k}^{2}} .
\end{gathered}
$$

Nach Professor Kamm [Kam+36] ist bekannt, dass die vektorielle Summe der Reifenlängsund -querkräfte auf einen Kreis beschränkt ist. Dies ist in Abbildung 3.1(b) dargestellt. 
Es handelt sich dabei um ein idealisiertes Modell, so dass in der Realität auch andere Formen der Beschränkung möglich sind (z.B. eine Ellipse). Nach dem zweiten Newtonschen Gesetz ist damit auch die Gesamtbeschleunigung auf einen Kreis beschränkt: $a_{\mathrm{tot}, k} \leq a_{\max }$.

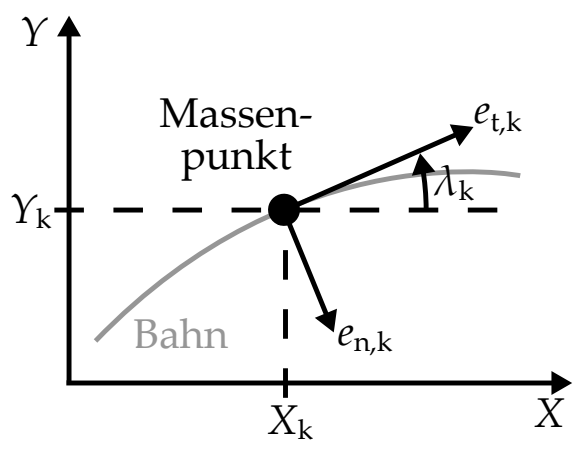

(a) Massenpunktmodell mit Richtungsvektoren in natürlichen Koordinaten.

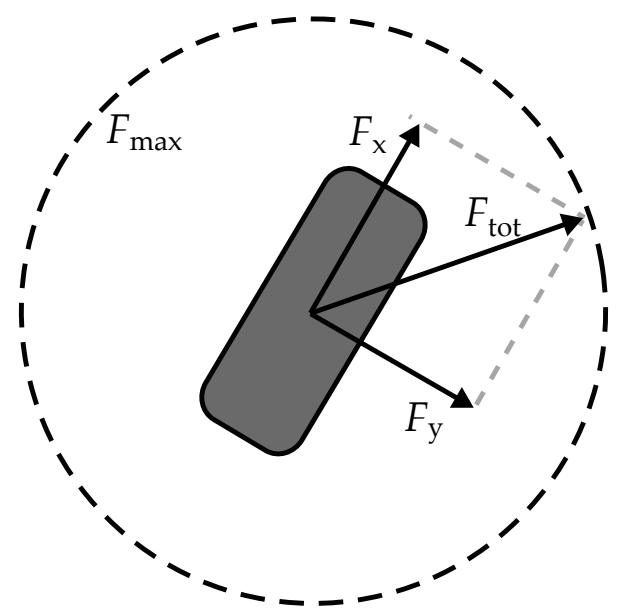

(b) Kräfte am Kammschen Kreis.

Abbildung 3.1.: Massenpunktmodell und Kammscher Kreis.

\subsection{Fahrdynamikmodelle}

\section{Reifenmodelle für kombinierten Schlupf}

Zwei häufig verwendete Reifenmodelle, die auch für gleichzeitiges Auftreten von Querund Längsschlupf ihre Gültigkeit nicht verlieren, sind das HSRI-Modell (siehe z.B. [Bra07]) und das Magic-Formula-Tire-Modell (siehe z.B. [Pac06]). Beide erfüllen die Forderung nach geringer Rechenlast. In [Göt14] wird gezeigt, dass sich das Pacejka Modell besser zur Parameteridentifikation eignet, da sich die Reifenkennlinien über einen weiten Bereich durch die Parameter des Modells verformen lassen. Für die Kraft in Reifenquerrichtung in Abhängigkeit des Schräglaufwinkels $\alpha=\tan \left(-\frac{v_{\mathrm{y}, \text { rad }}}{v_{\mathrm{x}, \mathrm{rad}}}\right)$ unter der Bedingung, dass der Längsschlupf verschwindet, gilt:

$$
F_{\mathrm{y} 0}(\alpha)=D_{\mathrm{y}} \sin \left(C_{\mathrm{y}} \arctan \left(B_{\mathrm{y}} \tan (\alpha)-E_{\mathrm{y}}\left(B_{\mathrm{y}} \tan (\alpha)-\arctan \left(B_{\mathrm{y}} \tan (\alpha)\right)\right)\right)\right) .
$$

Analog gilt in Abhängigkeit des Längsschlupfes

$$
s_{1}=\frac{\omega r_{\mathrm{dyn}}-v_{\mathrm{x}, \mathrm{rad}}}{\max \left(v_{\mathrm{x}, \mathrm{rad}}, \omega r_{\mathrm{dyn}}\right)}
$$

für die Kraft in Reifenlängsrichtung, wenn der Querschlupf verschwindet:

$$
F_{\mathrm{x} 0}\left(s_{1}\right)=D_{\mathrm{x}} \sin \left(C_{\mathrm{x}} \arctan \left(B_{\mathrm{x}} s_{1}-E_{\mathrm{x}}\left(B_{\mathrm{x}} s_{1}-\arctan \left(B_{\mathrm{x}} s_{1}\right)\right)\right)\right) .
$$


Im Falle des kombinierten Schlupfes treten Gewichtungsfaktoren für die Quer- und Längskräfte auf. Diese lauten

$$
\begin{aligned}
& G_{\mathrm{x}}\left(\alpha, s_{1}\right)=D_{\mathrm{a}} \cos \left(C_{\mathrm{a}}\left(\arctan \left(r_{\mathrm{Bx} 1}\left(\cos \left(\arctan \left(s_{1} r_{\mathrm{Bx} 2}\right)\right)\right) \alpha\right)\right)\right) \\
& G_{\mathrm{y}}\left(s_{1}, \alpha\right)=D_{\mathrm{s}} \cos \left(C_{\mathrm{s}}\left(\arctan \left(r_{\mathrm{By} 1}\left(\cos \left(\arctan \left(\alpha r_{\mathrm{By} 2}\right)\right)\right) s_{1}\right)\right)\right)
\end{aligned}
$$

Die Reifenkräfte ergeben sich demnach zu:

$$
F_{\mathrm{y}}\left(\alpha, s_{1}\right)=F_{\mathrm{y} 0}(\alpha) G_{\mathrm{y}}\left(s_{1}, \alpha\right) \text { und } F_{\mathrm{x}}\left(s_{1}, \alpha\right)=F_{\mathrm{x} 0}\left(s_{1}\right) G_{\mathrm{x}}\left(\alpha, s_{1}\right) .
$$

Die Gesamtkraft an einem Reifen ist die vektorielle Summe der Längs- und Querkraft:

$$
F_{\mathrm{tot}}=\sqrt{F_{\mathrm{x}}^{2}+F_{\mathrm{y}}^{2}}
$$

und ist in Abbildung 3.2 für die Vorderachse und die Hinterachse jeweils normiert auf die maximal übertragbare Kraft $F_{\max } \mathrm{zu}$ erkennen.

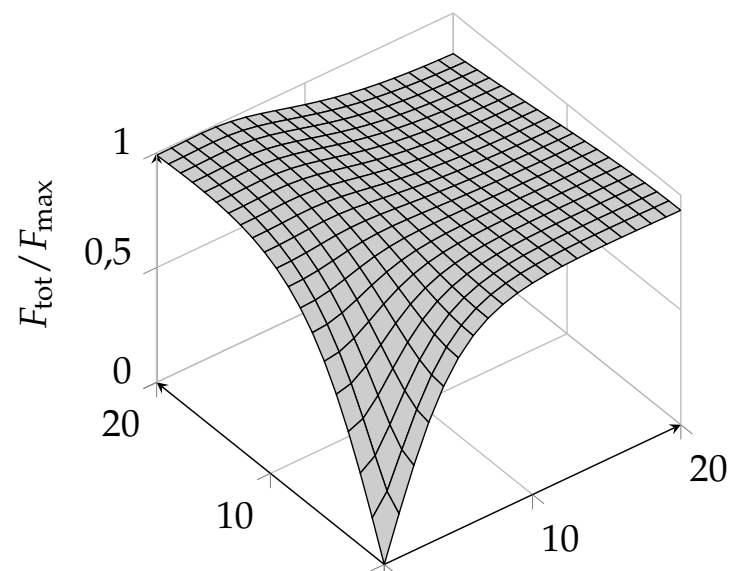

Schlupf [\%]
$0 \quad 0$

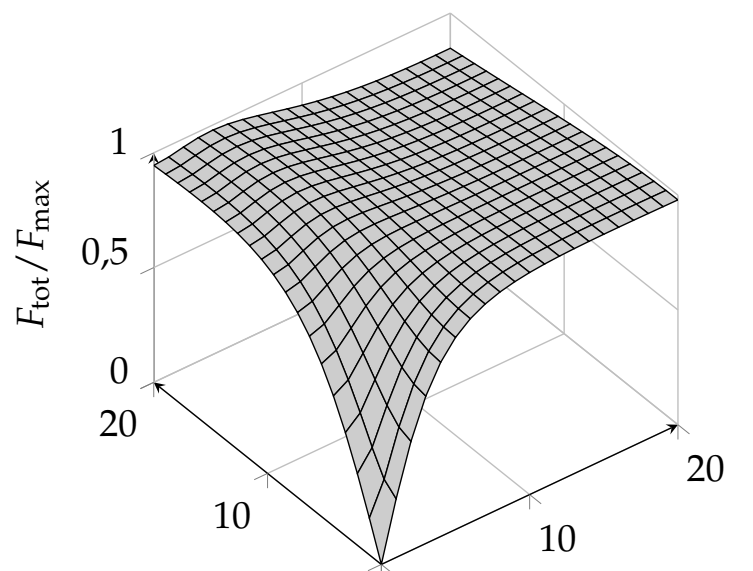

Schlupf $[\%]$
00

Schräglaufw. $\left[^{\circ}\right]$

Abbildung 3.2.: Kennfelder des Reifenmodells für die Vorderachse (links) und die Hinterachse (rechts) normiert auf die maximal übertragbare Kraft.

\section{Nichtlineares Einspurmodell}

Das nichtlineare Einspurmodell ist das einfachste dynamische Modell, welches zur Beschreibung der Fahrdynamik im Grenzbereich herangezogen werden kann. Zwar wird der Nick- und Wankfreiheitsgrad vernachlässigt, wodurch sich Abbildungsfehler ergeben, aber die für die ebene Bewegung wesentlichen Eigenschaften der Fahrdynamik werden beschrieben. Aufgrund der vergleichsweise geringen Modellkomplexität bleibt der Rechenaufwand in einem akzeptablen Rahmen. In Abbildung 3.3 ist das Einspurmodell schematisch in der Sicht von oben dargestellt. Die Zustandsdifferentialgleichungen ergeben sich in der Form

$$
\dot{\mathbf{x}}=\mathbf{f}(\mathbf{x}, \mathbf{u}), \mathbf{x}(t=0)=\mathbf{x}_{0}
$$




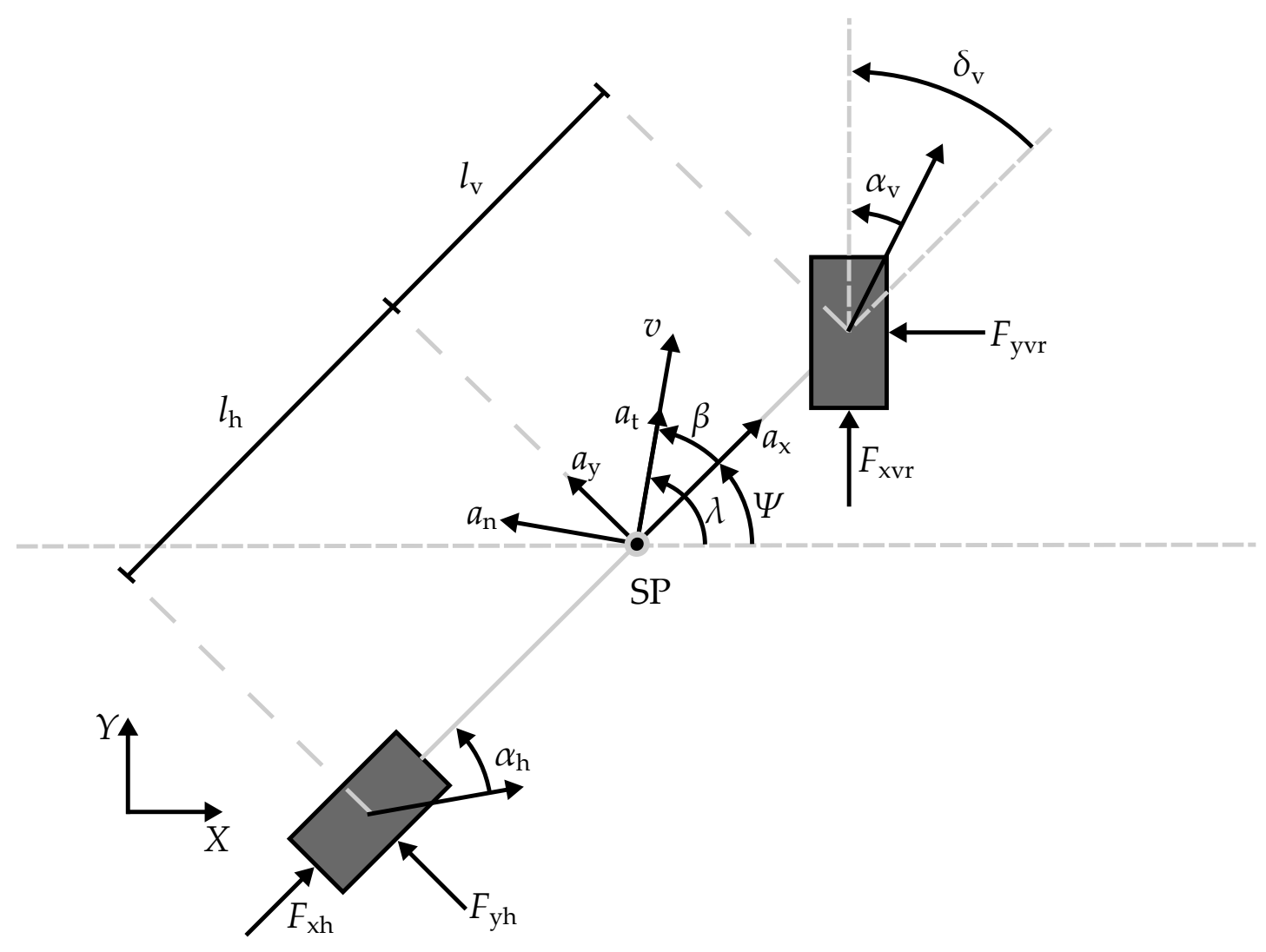

Abbildung 3.3.: Schematische Skizze des Einspurmodells.

mit

$$
\mathbf{x}=\left[\begin{array}{lll}
\beta & \dot{\Psi} & v
\end{array}\right]^{\mathrm{T}}, \mathbf{u}=\left[\begin{array}{ll}
\delta & s_{1}
\end{array}\right]^{\mathrm{T}} \text { und } \mathbf{f}(\mathbf{x}, \mathbf{u})=\left[\begin{array}{c}
\frac{a_{\mathrm{y}} \cos \beta-a_{\mathrm{x}} \sin \beta}{v}-\dot{\Psi} \\
\frac{l_{\mathrm{v}} F_{\mathrm{yv}}-l_{\mathrm{h}} F_{\mathrm{yh}}}{J_{\mathrm{z}}} \\
a_{\mathrm{y}} \sin \beta+a_{\mathrm{x}} \cos \beta
\end{array}\right] .
$$

Der Schlupf $s_{1}$ ist der mittlere Schlupf aller vier Reifen. Durch diese Wahl geht die Möglichkeit der radselektiven Bremsung verloren, da den vier unterlagerten Schlupfreglern der gleiche Sollschlupf übergeben wird. Bei der Anwendung modellprädiktiver Verfahren reduziert diese Maßnahme den Rechenaufwand und senkt die Komplexität. Der Radlenkwinkel $\delta_{\mathrm{v}}$ ergibt sich unter der vereinfachenden Annahme einer konstanten Lenkübersetzung $i_{\mathrm{s}} \mathrm{zu}: \delta_{\mathrm{v}}=\delta / i_{\mathrm{s}}$. Für die Beschleunigung in Fahrzeuglängs- bzw. -querrichtung gilt:

$$
a_{\mathrm{x}}=F_{\mathrm{x}, \text { ges }} / m \text { und } a_{\mathrm{y}}=F_{\mathrm{y}, \text { ges }} / \mathrm{m} \text {. }
$$

Die Gesamtkräfte ergeben sich aus der Summe der einzelnen Kräfte am Vorder- bzw. Hinterrad zu:

$$
F_{\mathrm{x}, \text { ges }}=F_{\mathrm{xv}}+F_{\mathrm{xh}} \text { und } F_{\mathrm{y}, \text { ges }}=F_{\mathrm{yv}}+F_{\mathrm{yh}} \text {. }
$$

Wobei die vorderen Reifenkräfte um den Radlenkwinkel gedreht werden müssen:

$$
F_{\mathrm{xv}}=F_{\mathrm{xvr}} \cos \delta_{\mathrm{v}}-F_{\mathrm{yvr}} \sin \delta_{\mathrm{v}} \text { und } F_{\mathrm{yv}}=F_{\mathrm{xvr}} \sin \delta_{\mathrm{v}}+F_{\mathrm{yvr}} \cos \delta_{\mathrm{v}} .
$$


Die Reifenquerkräfte sind abhängig von den Schräglaufwinkeln

$$
\alpha_{\mathrm{v}}=\delta_{\mathrm{v}}-\arctan \left(\frac{\dot{\Psi} l_{\mathrm{v}}+v \sin \beta}{v \cos \beta}\right), \alpha_{h}=\arctan \left(\frac{\dot{\Psi} l_{\mathrm{h}}-v \sin \beta}{v \cos \beta}\right)
$$

und dem Schlupf $s_{1}$ (Gleichung (3.2.2)):

$$
\begin{aligned}
F_{\mathrm{xvr}}= & D_{\mathrm{xv}} \sin \left(C_{\mathrm{xv}} \arctan \left(B_{\mathrm{xv}} s_{1}-E_{\mathrm{xv}}\left(B_{\mathrm{xv}} s_{1}-\arctan \left(B_{\mathrm{xv}} s_{1}\right)\right)\right)\right) G_{\mathrm{xvr}}, \\
F_{\mathrm{yvr}}= & D_{\mathrm{yv}} \sin \left(C _ { \mathrm { yv } } \operatorname { a r c t a n } \left(B_{\mathrm{yv}} \tan \left(\alpha_{\mathrm{v}}\right)+\cdots\right.\right. \\
& \left.\left.-E_{\mathrm{yv}}\left(B_{\mathrm{yv}} \tan \left(\alpha_{\mathrm{v}}\right)-\arctan \left(B_{\mathrm{yv}} \tan \left(\alpha_{\mathrm{v}}\right)\right)\right)\right)\right) G_{\mathrm{yvr}} \\
F_{\mathrm{xh}}= & D_{\mathrm{xh}} \sin \left(C_{\mathrm{xh}} \arctan \left(B_{\mathrm{xh}} s_{1}-E_{\mathrm{xh}}\left(B_{\mathrm{xh}} s_{1}-\arctan \left(B_{\mathrm{xh}} s_{1}\right)\right)\right)\right) G_{\mathrm{xh}}, \\
F_{\mathrm{yh}}= & D_{\mathrm{yh}} \sin \left(C _ { \mathrm { yh } } \operatorname { a r c t a n } \left(B_{\mathrm{yh}} \tan \left(\alpha_{\mathrm{h}}\right)+\cdots\right.\right. \\
& \left.\left.-E_{\mathrm{yh}}\left(B_{\mathrm{yh}} \tan \left(\alpha_{\mathrm{h}}\right)-\arctan \left(B_{\mathrm{yh}} \tan \left(\alpha_{\mathrm{h}}\right)\right)\right)\right)\right) G_{\mathrm{yh}} .
\end{aligned}
$$

Die Gewichtungsfunktionen zur Abbildung der Reifeneigenschaften bei gleichzeitigem Längs- und Querschlupf lauten:

$$
\begin{aligned}
G_{\mathrm{xvr}} & =D_{\mathrm{a}} \cos \left(C_{\mathrm{a}}\left(\arctan \left(r_{\mathrm{Bx} 1}\left(\cos \left(\arctan \left(s_{1} r_{\mathrm{Bx} 2}\right)\right)\right) \alpha_{\mathrm{v}}\right)\right)\right), \\
G_{\mathrm{yvr}} & =D_{\mathrm{s}} \cos \left(C_{\mathrm{s}}\left(\arctan \left(r_{\mathrm{By} 1}\left(\cos \left(\arctan \left(\alpha_{\mathrm{v}} r_{\mathrm{By} 2}\right)\right)\right) s_{1}\right)\right)\right), \\
G_{\mathrm{xh}} & =D_{\mathrm{a}} \cos \left(C_{\mathrm{a}}\left(\arctan \left(r_{\mathrm{Bx} 1}\left(\cos \left(\arctan \left(s_{1} r_{\mathrm{Bx} 2}\right)\right)\right) \alpha_{\mathrm{h}}\right)\right)\right), \\
G_{\mathrm{yh}} & =D_{\mathrm{s}} \cos \left(C_{\mathrm{s}}\left(\arctan \left(r_{\mathrm{By} 1}\left(\cos \left(\arctan \left(\alpha_{\mathrm{h}} r_{\mathrm{By} 2}\right)\right)\right)_{1}\right)\right)\right) .
\end{aligned}
$$

Zur Identifikation der Parameter bieten sich quasistationäre Kreisfahrten im fahrdynamischen Grenzbereich an. Ohne Antriebs- und Bremsmomente (daraus resultiert $s_{1}=0$ ) wird hierzu der Lenkradwinkel langsam erhöht. Die Geschwindigkeit wird als veränderlicher Parameter auf das Modell gegeben und die Gierrate und die Querbeschleunigung werden als Vergleichsgrößen berechnet. Um eine gleichwertige Abbildungsgenauigkeit für die Gierrate und die Querbeschleunigung zu erreichen, wird das normierte Gütemaß

$$
J_{\mathrm{ESM}}=J_{\dot{\Psi}}+J_{\mathrm{a}, \mathrm{y}}
$$

mit

$$
J_{\dot{\Psi}}=\sum_{k=1}^{N} \begin{cases}\left(\frac{\dot{\Psi}_{\text {mess }, k}-\dot{\Psi}_{\text {sim }, k}}{\dot{\Psi}_{\text {mess }, k}}\right)^{2} & \text { wenn }\left|\dot{\Psi}_{\text {mess }, k}\right| \geq \dot{\Psi}_{\text {lim }} \\ 0 & \text { sonst }\end{cases}
$$

und

$$
J_{\mathrm{a}, \mathrm{y}}=\sum_{k=1}^{N} \begin{cases}\left(\frac{a_{\mathrm{y}, \text { mess }, k}-a_{\mathrm{y}, \text { sim }, k}}{a_{\mathrm{y}, \text { mess }, k}}\right)^{2} & \text { wenn }\left|a_{\mathrm{y}, \text { mess }, k}\right| \geq a_{\mathrm{y}, \text { lim }} \\ 0 & \text { sonst }\end{cases}
$$

verwendet. Mit dieser Methode können die Reifenkennlinien in Querrichtung, die Schwerpunktlage, die Masse und das Trägheitsmoment um die Hochachse identifiziert werden. Die Reifenkennlinien in Längsrichtung und die Koppelterme werden mit aus der Literatur [Pac06] bekannten Werten parametriert. Zur Validierung werden Notausweichmanöver verwendet, da das Modell in erster Linie bei diesen Manövern verwendet werden soll und die Wankdynamik einen größeren Einfluss hat als bei stationären Kreisfahrten. Somit kann der im Einspurmodell inhärente Modellfehler 
abgeschätzt werden. Der Verlauf von Mess- und Simulationsgrößen ist in Abbildung 3.4 dargestellt.
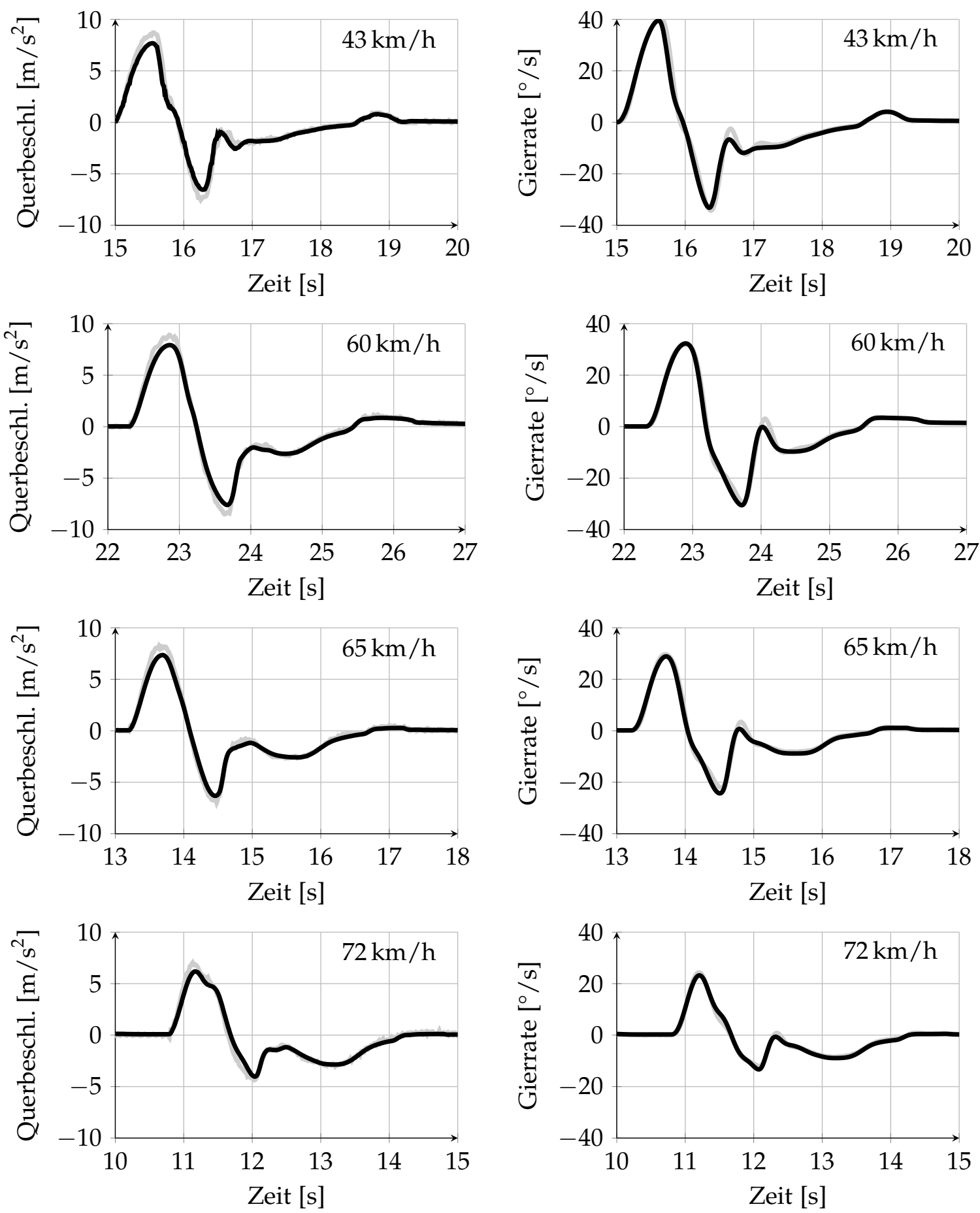

Abbildung 3.4.: Vergleich simulierter (schwarz) und gemessener (grau) Verläufe der Gierrate und der Querbeschleunigung bei Spurwechselmanövern. Die initialen Geschwindigkeiten $v_{\text {init }}$ sind jeweils angegeben.

Es handelt sich um Manöver, welche bei ca. 40 bis ca. $72 \mathrm{~km} / \mathrm{h}$ durchgeführt wurden. Für die Querbeschleunigung wird ein Fit-Wert von fit $=87,20 \%$ und für die Gierrate ein Fit-Wert von fit $=89,31$ \% erreicht (siehe Gleichung A.1.17)). Die Abbildungsgenauigkeit ist für beide Größen vergleichbar, was das Ziel der Formulierung 
des Gütemaßes (3.2.22) ist. In [Buß13] werden bei dem Vergleich eines nichtlinearen Einspurmodells mit einem kommerziellen Mehrkörpersimulationsprogramm ähnliche Ergebnisse erzielt.

\section{Ebenes Zweispurmodell}

Das ebene Zweispurmodell weist eine höhere Komplexität und Rechenlast auf als das Einspurmodell. Der wesentliche Vorteil besteht in der Möglichkeit, radselektive Bremseingriffe zu betrachten. Das ebene Zweispurmodell ist ein nichtlineares Mehrgrößensystem der gleichen Form, wie das nichtlineare Einspurmodell (3.2.8) bis auf den Eingangsvektor, der fünf Stellgrößen umfasst:

$$
\mathbf{u}=\left[\begin{array}{lllll}
\delta & s_{\mathrm{vl}} & s_{\mathrm{vr}} & s_{\mathrm{hl}} & s_{\mathrm{hr}}
\end{array}\right]^{\mathrm{T}}
$$

Aus Gründen der Übersichtlichkeit wird hier auf die Angabe der Zustandsdifferentialgleichungen verzichtet. Entsprechende Abhandlungen finden sich in der Literatur (siehe z.B. [Mit03]).

\subsection{Modellierung der Aktuatorregelkreise}

Der Lenkwinkelregelkreis aus Abbildung 2.2 weist eine Dynamik auf, welche gegenüber der Fahrdynamik nicht zu vernachlässigen ist. Bei Betrachtung der Sprungantwort aus Abbildung 2.3 ist offensichtlich, dass es sich mindestens um ein System zweiter Ordnung handeln muss. Weiterhin muss die Begrenzung der Lenkrate berücksichtigt werden. Diese beiden Überlegungen führen auf die Zustandsdifferentialgleichung:

$$
T_{\mathrm{LWR}}^{2} \ddot{\delta}+2 d_{\mathrm{LWR}} T_{\mathrm{LWR}} B_{\mathrm{LWR}}(\dot{\delta})+\delta=k_{\mathrm{LWR}} \delta_{\text {soll }}
$$

mit

$$
B_{\text {LWR }}= \begin{cases}\dot{\delta}_{\text {min }} & \text { für } \dot{\delta}<\dot{\delta}_{\min } \\ \dot{\delta} & \text { für } \dot{\delta}_{\min }<\dot{\delta}<\dot{\delta}_{\max } . \\ \dot{\delta}_{\max } & \text { für } \dot{\delta} \dot{\delta}_{\max }\end{cases}
$$

Auch für die Modelle der Aktuatorregelkreise gelten die Forderungen nach einer hohen Abbildungsgenauigkeit und geringen Rechenlast. Das obige Modell stellt, unter der Berücksichtigung der oben beschriebenen offensichtlichen Modelleigenschaften, das Modell mit der geringsten Ordnung dar. Es wird im Folgenden gezeigt, dass die Abbildungsgenauigkeit ausreichend ist. Hierzu werden die Parameter identifiziert und auf den in Abbildung 3.5 gezeigten unangesehenen Daten wird ein Fit-Wert von fit = $94,7744 \%$ erreicht. In modernen Fahrzeugen werden Bremsschlupfregelungssysteme eingesetzt, um die Funktionen ESC und ABS zu realisieren. In Abbildung 3.6 sind Sprungantworten des geschlossenen Schlupfregelkreises des Fahrsimulators dargestellt. Um wiederum ein Modell mit möglichst geringer Komplexität zu erhalten, wird ein lineares System erster Ordnung angesetzt:

$$
T_{\mathrm{s}} \dot{s}_{1}+s_{1}=k_{\mathrm{s}} s_{\mathrm{soll}}
$$

Die Parameter $T_{s}$ und $k_{s}$ werden identifiziert und die Abbildungsgenauigkeit des Modells überprüft. In Abbildung 3.6 wird der gemessene mit dem simulierten Schlupf 

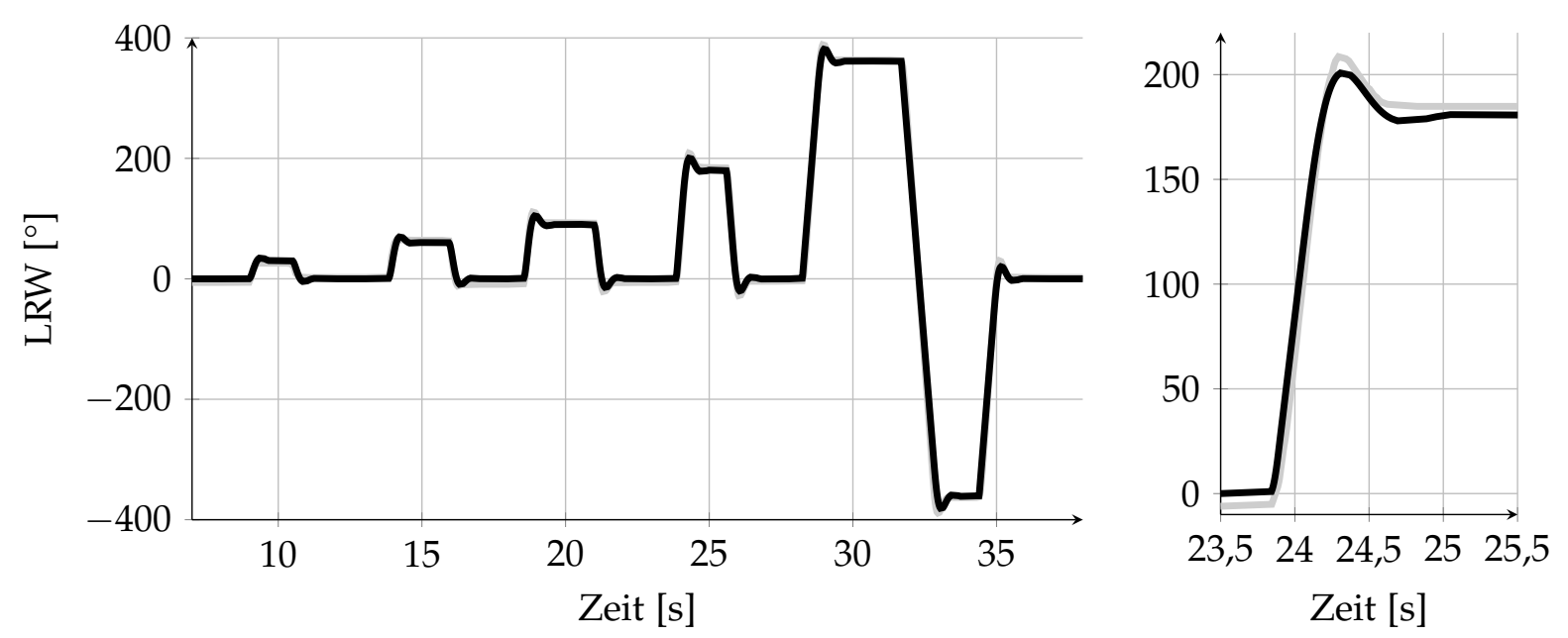

Abbildung 3.5.: Vergleich simulierter (schwarz) und gemessener (grau) Verläufe des Lenkradwinkels (LRW).
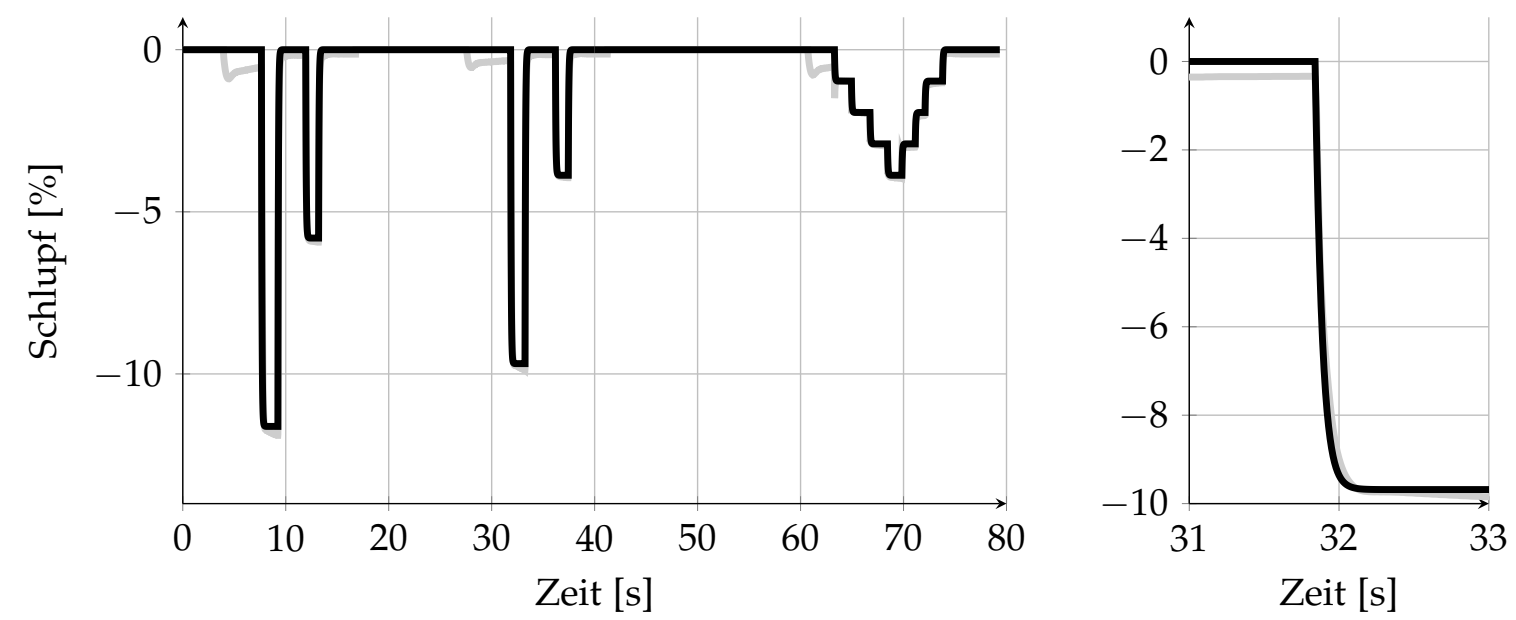

Abbildung 3.6.: Vergleich simulierter (schwarz) und gemessener (grau) Verläufe des Schlupfes.

verglichen. Der Fit-Wert beträgt $f$ it $=89,24 \%$. Zwischen den Bremsmanövern wurde das Fahrzeug manuell beschleunigt. Der Schlupfregler greift ausschließlich über die Bremse ein. Es ist daher nicht möglich, Antriebsschlupf zu erzeugen. Dementsprechend wurden die Signalverläufe vor der Identifikation auf negative Werte begrenzt. Deutliche Abweichungen zwischen simulierten und gemessenen Werten ergeben sich im Schleppbetrieb (bzw. Rekuperation), wenn der Regler nicht aktiviert ist. Dies hat jedoch keine praktische Relevanz für Notmanöver.

\subsection{Auswahl des vollständigen Prädiktionsmodells}

In Abbildung 3.7 ist eine Ordnung der verschiedenen Abstraktionsgrade von Fahrzeugmodellen nach Komplexität und Rechenlast zu erkennen. Es ist gekennzeichnet, welche Modelle sich als Prädiktionsmodell eignen. Das lineare Einspurmodell kommt für automatisierte Fahrfunktionen in Frage. Es gilt jedoch nicht im Grenzbereich der Fahrdynamik und eignet sich daher nicht für Notausweichmanöver. An ein Prädiktionsmodell werden die folgenden zentralen Anforderungen gestellt: 


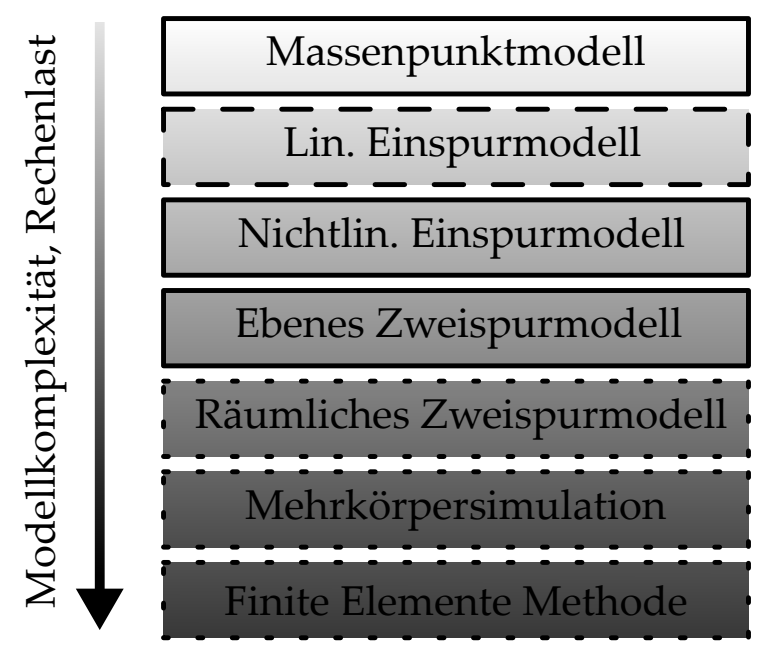

Abbildung 3.7.: Verschiedene Abstraktionsgrade von Fahrdynamikmodellen geordnet nach Modellkomplexität und Rechenlast. Die durch gepunktete Linien eingerahmten Modelle eignen sich aufgrund der hohen Rechenlast nicht als Prädiktionsmodell. Das mit gestrichelten Linien eingerahmte lineare Einspurmodell kann nicht für Notmanöver verwendet werden.

- Ausreichende Abbildungsgenauigkeit und

- geringe Rechenlast.

Zwischen diesen beiden Anforderungen besteht ein Zielkonflikt. Das Prädiktionsmodell bestimmt darüber hinaus die Eingriffsmöglichkeiten des modellprädiktiven Verfahrens, welche sich wie folgt gliedern lassen:

- ausschließlich Bremseingriff

- ausschließlich Lenkeingriffe

- Lenkeingriff an einer Achse

- Lenkeingriff an zwei Achsen

- kombinierter Lenk- und Bremseingriff

- Lenk- und nicht radselektiver Bremseingriff

- Lenk- und radselektiver Bremseingriff

Es ist offensichtlich, dass ein Notausweichmanöver einen Lenkeingriff erfordert. Die meisten Fahrzeuge verfügen über ein Lenksystem an der Vorderachse, aber nicht an der Hinterachse. Eine aktive Hinterachslenkung wird daher in dieser Arbeit nicht betrachtet. Diese zusätzliche Eingriffsmöglichkeit erfordert jedoch nicht zwingend eine weitere Eingangsgröße des Prädiktionsmodells, da der hintere Lenkwinkel in einfacher Weise an den vorderen gekoppelt werden kann. Dies kann in Abhängigkeit der Geschwindigkeit gegensinnig oder gleichsinnig erfolgen. Der Längseingriff ermöglicht das Kollisionsvermeidungspotential des Fahrzeugs auszunutzen. In [Sch+06a] wird gezeigt, dass das optimale Ausweichmanöver einen kombinierten Längs- und Quereingriff erfordert. Der radselektive Bremseingriff ermöglicht eine bessere Ausnutzung des gierdynamischen Potentials. Allerdings bedingt ein Prädiktionsmodell mit 
fünf Eingangsgrößen und zwölf Zustandsgrößen einen erheblichen Rechenaufwand und erhebliche Komplexität. Da die Komplexität der modellprädiktiven Planung und Regelung ohnehin im Vergleich die höchste ist, ist es zielführend, nur eine längsdynamische Eingangsgröße zu wählen. Zunächst liegt es nahe, den Bremsdruck oder einen Bremspedalwert zu wählen. Dies führt jedoch dazu, dass das komplexe und nichtlineare Verhalten der Bremsanlage, der Reibwertpaarung Bremsscheibe-Bremsbelag und der Reifen zu modellieren wären. Zudem sind die Zeitkonstanten des Bremssystems im Vergleich zur Fahrdynamik klein, wodurch sich eine höhere Anforderung an die Abtastzeit ergibt. Es ist daher vorteilhaft, einen unterlagerten Schlupfregler zu verwenden, welcher eine einfacher modellierbare Dynamik des geschlossenen Regelkreises bewirkt. Schlupfregelungssysteme sind weit verbreitet [Ise06] und sollen hier nicht weiter erläutert werden. In [Göt14], [Göt+15] und [Göt+16b] wird eine Untersuchung des MPPC bei Verwendung eines ebenen Zweispurmodells durchgeführt. Es wird gezeigt, dass sich kollisionsfreie Notmanöver und auch automatisierte Fahrfunktionen darstellen lassen. Jedoch enthält das Gütemaß sehr viele Anteile mit entsprechend vielen Gewichtungen, welche aufgrund der Komplexität nur mit erheblichem Aufwand gewählt werden können. Die Ergebnisse zeigen zudem nicht, dass die Möglichkeit mittels radselektiver Bremsung im großen Maße ausgenutzt wird, was sich mit dem Ergebnis aus [Fra+13] deckt. Neben einem geeigneten Fahrdynamikmodell sind zwingend die Aktuatorregelkreise zu berücksichtigen, wenn mit einem modellprädiktiven Verfahren die integrierte Planung und Regelung durchgeführt werden soll. Dies wird offensichtlich, wenn die Zeitkonstanten der einzelnen Modelle verglichen werden. Die Summenzeitkonstante der Gierübertragungsfunktion liegt je nach Modell und Fahrgeschwindigkeit im Bereich $T_{\Sigma}=50 . .200 \mathrm{~ms}$. Die Zeitkonstante des Lenkwinkelregelkreises beträgt $T_{\mathrm{LWR}}=116,3 \mathrm{~ms}$ und die des Schlupfregelkreises $T_{\mathrm{S}}=52,1 \mathrm{~ms}$. Um das Modell zu vervollständigen, wird die Position in globalen Koordinaten aus den Differentialgleichungen

$$
\dot{Y}=v \sin (\beta+\Psi) \text { und } \dot{X}=v \cos (\beta+\Psi)
$$

bestimmt. Beide Gleichungen besitzen keine Parameter, die identifiziert werden müssen. Die Startwerte der Integratoren ergeben sich aus der Wahl des Koordinatensystemursprungs. Entsprechend ist auch keine Validierung notwendig. In Abbildung 3.8 ist das Blockschaltbild des vollständigen Prädiktionsmodells, wie es in dieser Arbeit verwendet wird, dargestellt. Die vier Anteile sind unabhängig voneinander. Die Unabhängigkeit der Aktuatorregelungssysteme von der Fahrdynamik resultiert aus der Annahme, dass die entsprechenden Regler fahrdynamische Abhängigkeiten ausregeln.

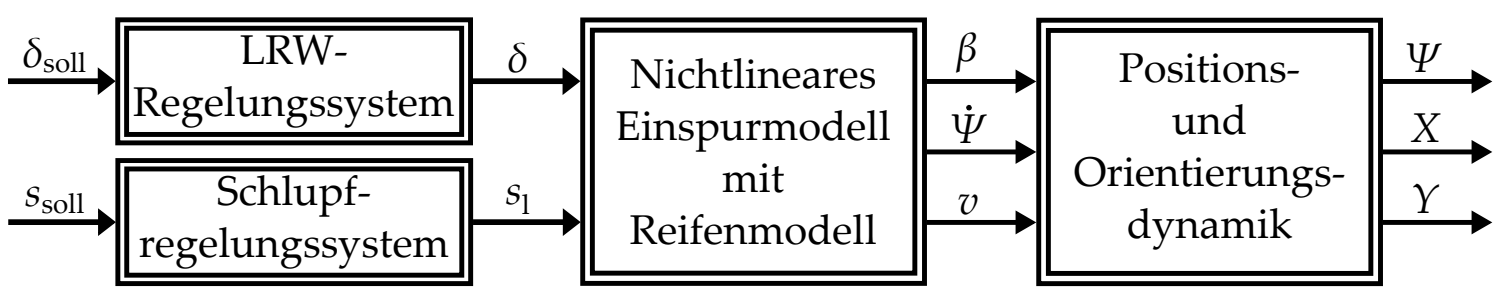

Abbildung 3.8.: Blockschaltbild des vollständigen Prädiktionsmodells. 


\section{4}

Bahnfolgeverfahren

Das Bahnfolgeverfahren besteht im Wesentlichen aus drei Komponenten: Planung der Ausweichbahn, Eingriffsentscheidung und Folgeregelung auf die Bahn. In Abbildung 4.1 ist die Architektur des Verfahrens dargestellt. Basierend auf den Umfeldinfor-

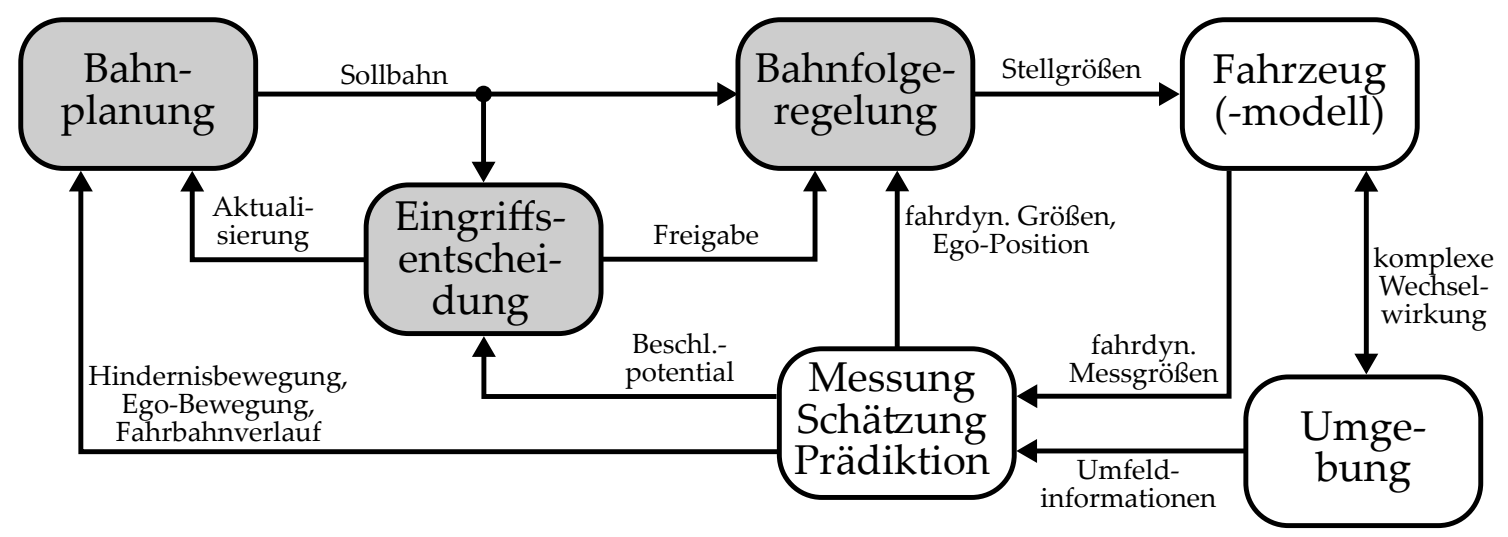

Abbildung 4.1.: Architektur des Bahnfolgeverfahrens.

mationen wird die kollisionsfreie Ausweichbahn berechnet. Diese wird durch die Eingriffsentscheidung bewertet und als Sollgröße an den Positionsregler weitergegeben, welcher die Abweichung der eigenen Fahrzeugposition von dem Sollwert ausregelt, sobald die Situation als kritisch klassifiziert wurde (Freigabe). Gleichzeitig wird die Aktualisierung der Bahnplanung gestoppt. Die Bahnfolgeregelung ist abhängig vom fahrdynamischen Zustand und gibt die Stellgrößen auf das Fahrzeug, welches mit der Umgebung in einer komplexen Wechselwirkung agiert. Einflussfaktoren sind z.B. der Reibwert des Rad-Straße-Kontaktes und die Bewegungen anderer Verkehrsteilnehmer, welche wiederum auf die Bewegung des Ego-Fahrzeugs reagieren. Die fahrdynamischen Zustandsgrößen werden entweder messtechnisch oder durch Beobachterstrukturen rekonstruiert. Hierzu wird neben Kamera und Radar auch die übliche Fahrdynamiksensorik aus dem ESC verwendet. Der Schwimmwinkel wird im Versuchsfahrzeug durch ein Extended Kalman Filter und ein nichtlineares Einspurmodell beobachtet. Wegen der hohen Dynamik während des Ausweichmanövers ist die Positionsmessung mit Hilfe der Kamera nicht zuverlässig. Während des Spurwechsels wird die Position des Ego-Fahrzeugs daher basierend auf den gemessenen und rekonstruierten Fahrdynamikgrößen berechnet. 


\subsection{Beschreibung von Ausweichbahnen durch Funktionen}

Die Bahnplanung erfolgt durch Geraden (Spurhalten) und einem Polynom 5. Grades (Spurwechsel). Polynome lassen sich sehr einfach aufgrund der Randbedingungen der Verkehrssituation berechnen. Das Polynom 5. Grades stellt dabei einen Kompromiss zwischen minimaler Krümmung und Realisierbarkeit dar. Die Krümmung ist verglichen mit einem kubischen Polynom höher, jedoch ergibt sich durch die zweifach stetige Differenzierbarkeit an den Übergangsstellen von der Geraden auf das Polynom eine bessere Fahrbarkeit bzw. Realisierbarkeit, da der Krümmungsverlauf stetig ist. Bei der Verwendung von Polynomen 3. Grades hingegen treten an den Übergangsstellen Unstetigkeiten im Krümmungsverlauf auf. Polynome höheren Grades weisen höhere Krümmungen auf und sind aufwändiger zu berechnen. In Abbildung 4.2 ist die Bahnplanung skizziert. Ab dem Zeitpunkt der Auslösung wird die Bahnplanung

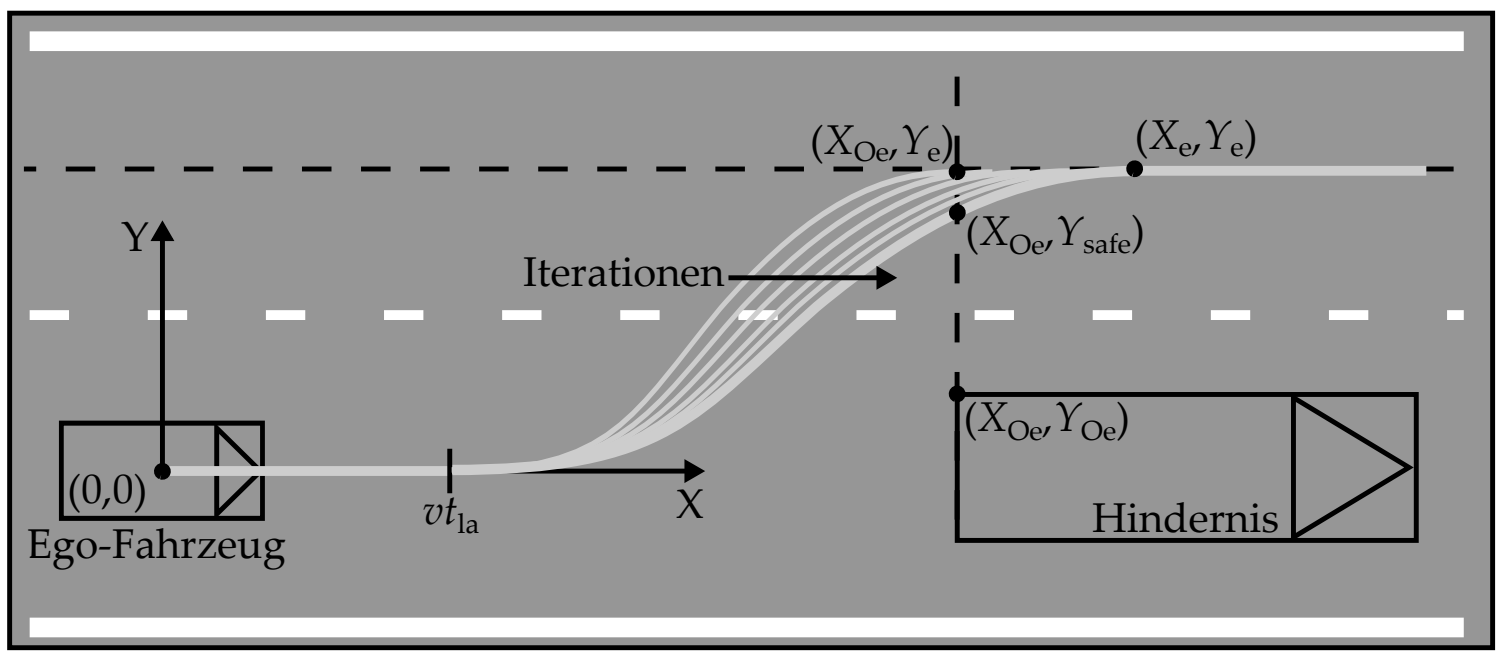

Abbildung 4.2.: Bahnplanung für die zu Grunde gelegte Verkehrssituation.

nicht mehr aktualisiert. Die Bahn beginnt mit einem Geradenstück der Länge $v t_{\text {la, }}$ bevor sich das Polynom anschließt. Auf den ersten Blick erscheint dieses Vorgehen nachteilig, da Zeit zum Ausweichen „verschenkt" wird. Dieser "Vorlauf" entspricht jedoch der Vorausschauzeit des Positionsreglers. Auf diese Weise kann die Sollbahn mit hoher Präzision abgefahren werden. Ohne das Geradenstück zu Beginn ergäbe sich ein Sprung der Regelabweichung am Vorausschaupunkt zu Beginn des Manövers. Das Polynom ist gegeben durch:

$$
Y_{p o l}(X)=a_{5} X^{5}+a_{4} X^{4}+a_{3} X^{3}+a_{2} X^{2}+a_{1} X+a_{0} .
$$

Zunächst werden die Koeffizienten durch die Randbedingungen

$$
\begin{aligned}
& Y_{\text {pol }}\left(v t_{\text {la }}\right)=0, \quad Y_{\text {pol }}\left(X_{\text {Oe }}\right)=Y_{\mathrm{e}}, \\
& Y_{\text {pol }}^{\prime}\left(v t_{\mathrm{la}}\right)=0, \quad Y_{\text {pol }}^{\prime}\left(X_{\text {Oe }}\right)=0 \text {, } \\
& Y_{\text {pol }}^{\prime \prime}\left(v t_{\mathrm{la}}\right)=0 \quad \text { und } \quad Y_{\text {pol }}^{\prime \prime}\left(X_{\text {Oe }}\right)=0
\end{aligned}
$$

bestimmt, wobei $Y_{\mathrm{e}}$ der Mitte der freien Fahrbahn entspricht. Diese initiale Bahn wird anschließend iterativ an die Situation angepasst, indem $X_{\text {Oe }}$ schrittweise erhöht wird, 
bis die Bahn den Sicherheitsabstand zum Hindernis erreicht oder unterschreitet (siehe $[\overline{\mathrm{Ha} \beta}+14])$. Die finale Ausweichbahn ergibt sich aus der vorletzten Iteration $\left(X_{\mathrm{Oe}}=X_{\mathrm{e}}\right)$. Ein weiterer Vorteil dieses Verfahrens ist die geringe Rechenlast. Der wesentliche Nachteil besteht darin, dass sich das Verfahren schlecht an andere Verkehrssituationen mit z.B. mehreren Hindernissen anpassen lässt.

\subsection{Kritikalitätsmaß und Eingriffsentscheidung}

Da es sich um ein Notfallsystem handelt, welches nicht permanent in die Fahrzeugführung eingreift, wird eine Eingriffsentscheidung benötigt. Das heißt, dass Bedingungen formuliert werden müssen, bei deren Erfüllung das System mit Eingriffen beginnt und auch wieder aufhört. Um ein autonomes Manöver auszulösen, muss eine kritische Situation vorliegen. Die Klassifikation einer Situation als kritisch ist abhängig vom Trajektorienplanungsverfahren und wird in Abschnitt A.1.3 diskutiert. Das assistierte Manöver muss durch den Fahrer ausgelöst werden. Die hierfür nötigen Bedingungen sind:

- Die vorliegende Verkehrssituation ist kritisch.

- Das vom Fahrer aufgebrachte Handmoment, die Lenkrate und der Lenkwinkel überschreiten Grenzwerte.

Die zweite Bedingung wird als eine deutliche Lenkbewegung verstanden. Der Eingriff des Systems wird beendet, wenn die folgenden Bedingungen erfüllt sind:

- Der fahrdynamische Zustand ist stabil.

- Die Regelabweichung zur Spurmitte liegt unter einem Grenzwert.

- Die Zeit seit der Auslösung des Manövers hat einen unteren Grenzwert überschritten.

Da der Fahrer das Fahrzeug möglicherweise dauerhaft außerhalb der Fahrbahnmitte führt, steigt das System aus der Regelung aus, wenn folgende Bedingungen erfüllt sind:

- Der fahrdynamische Zustand ist stabil.

- Die vergangene Zeit seit der Auslösung überschreitet einen oberen Grenzwert.

Das aus der Entwicklung der Notbremsfunktionen (z.B. AEB) bekannte Maß der TTC (Time To Collision) ist für Notausweichmanöver nur bedingt geeignet, da es die relative Position in Straßenquerrichtung von Hindernis, Ego-Fahrzeug und Fahrbahnbegrenzung nicht in Betracht zieht. Zur Approximation der Kritikalität basierend auf dem Nutzungsgrad des Bewegungspotentials (siehe Definition A.1.14) bietet es sich an, die geplante Bahn zu untersuchen. Die kinematischen Größen, welche entlang der Bahn berechnet werden können, stellen eine Prädiktion der Verläufe der fahrkinematischen Größen im Sinne des Massenpunktmodells dar (siehe Kapitel 3). Da das Massenpunktmodell die Kursrate bzw. den Kurswinkel nicht in Schwimmwinkeländerung 
bzw. Schwimmwinkel und Gierrate bzw. Gierwinkel unterscheiden kann, bleiben als fahrdynamische Größen die Kursrate, der Kurswinkel und die Querbeschleunigung. Die maximal erreichbaren Kursraten bzw. Kurswinkel sind von Fahrzeug zu Fahrzeug unterschiedlich und hängen vom Reibwert und der Geschwindigkeit ab. Die maximal erreichbare Querbeschleunigung variiert weniger stark von Fahrzeug zu Fahrzeug, ist nahezu unabhängig von der Geschwindigkeit und skaliert näherungsweise linear mit dem Reibwert. Zur Approximation der Kritikalität wird daher die maximale Querbeschleunigung, welche ein Massenpunkt erfährt, wenn er mit unveränderter Geschwindigkeit entlang der geplanten Bahn bewegt wird, verwendet: $K_{\text {pot }}=a_{\mathrm{y}, \max } / \mathrm{g}$. Überschreitet diese Größe einen Grenzwert $K_{\text {pot }}=1$, wird die Situation als kritisch angesehen. In Abbildung 4.3 ist der Verlauf der maximalen Querbeschleunigung bei der Anfahrt auf ein Hindernis dargestellt. Unterhalb der unteren gestrichelten hori-

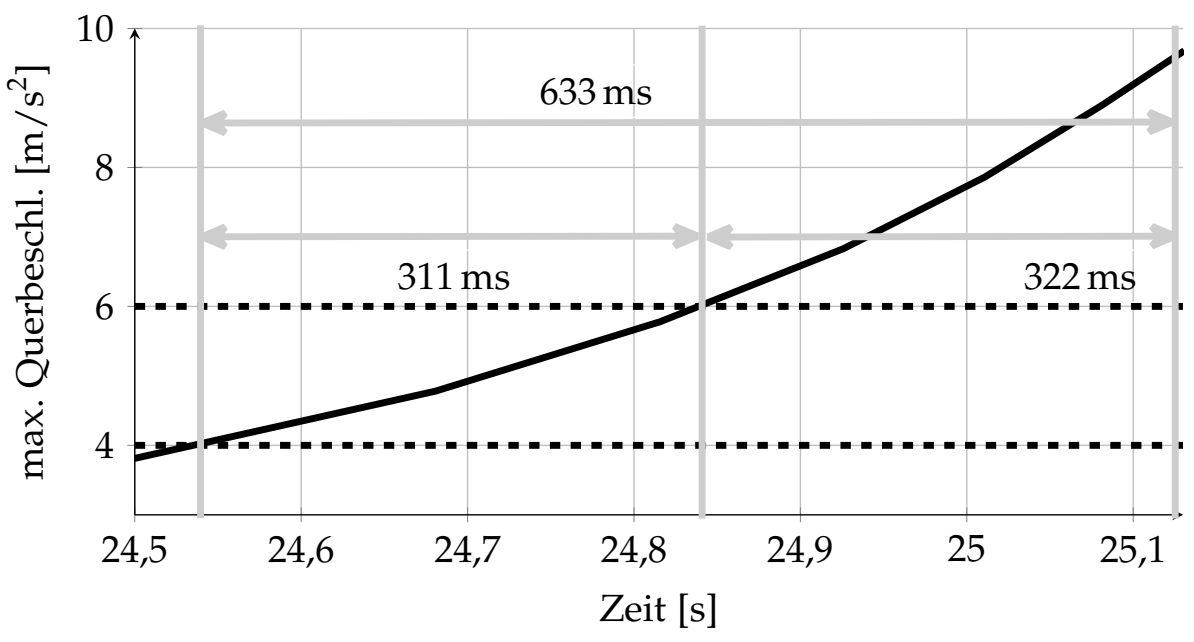

Abbildung 4.3.: Verlauf der maximalen Querbeschleunigung (schwarz und durchgezogen) bei der Anfahrt auf ein Hindernis. Die gestrichelten Linien zeigen die Grenzen des linearen, des Übergangs- und des nichtlinearen Bereiches an. Die beschrifteten Pfeile kennzeichnen die Zeiträume zwischen den Bereichen.

zontalen Linie befindet sich der lineare Bereich der Fahrdynamik. Die meisten Fahrer halten sich während der normalen Fahrt in diesem Bereich auf und kennen daher die Fahrzeugreaktionen. Zwischen den beiden gestrichelten Linien befindet sich der Übergangsbereich. Einige Fahrzeuge verhalten sich hier noch weitgehend linear, andere bereits nichtlinear. Nur wenige Fahrer verwenden diesen Bereich und wenn, dann nur selten, sodass ihnen das Fahrverhalten in diesem Bereich wenig vertraut ist. Oberhalb der oberen gestrichelten Linie beginnt der Grenzbereich, in dem sich das Fahrverhalten nichtlinear verhält. Dieser Bereich wird bei verantwortungsvoller Fahrweise im normalen Straßenverkehr nicht erreicht. Nur wenige professionelle Fahrer sind mit den nichtlinearen Fahrzeugreaktionen vertraut. Es ist zu erkennen, dass die Kritikalität des geplanten Manövers innerhalb von ca. 633 ms von einem Manöver, welches im linearen Bereich der Fahrdynamik durchführbar ist, zu einem Manöver, welches das Querbeschleunigungspotential des Fahrzeugs ausnutzt, steigt. 


\subsection{Fahrzeugquerregelung}

Dieser Abschnitt beschreibt die Fahrzeugquerregelung beginnend mit der generellen Struktur, bevor die einzelnen Komponenten sukzessive erläutert werden. In Abbildung 4.4 ist das Blockschaltbild des Querreglers dargestellt. Die Auswahlstrategie wählt

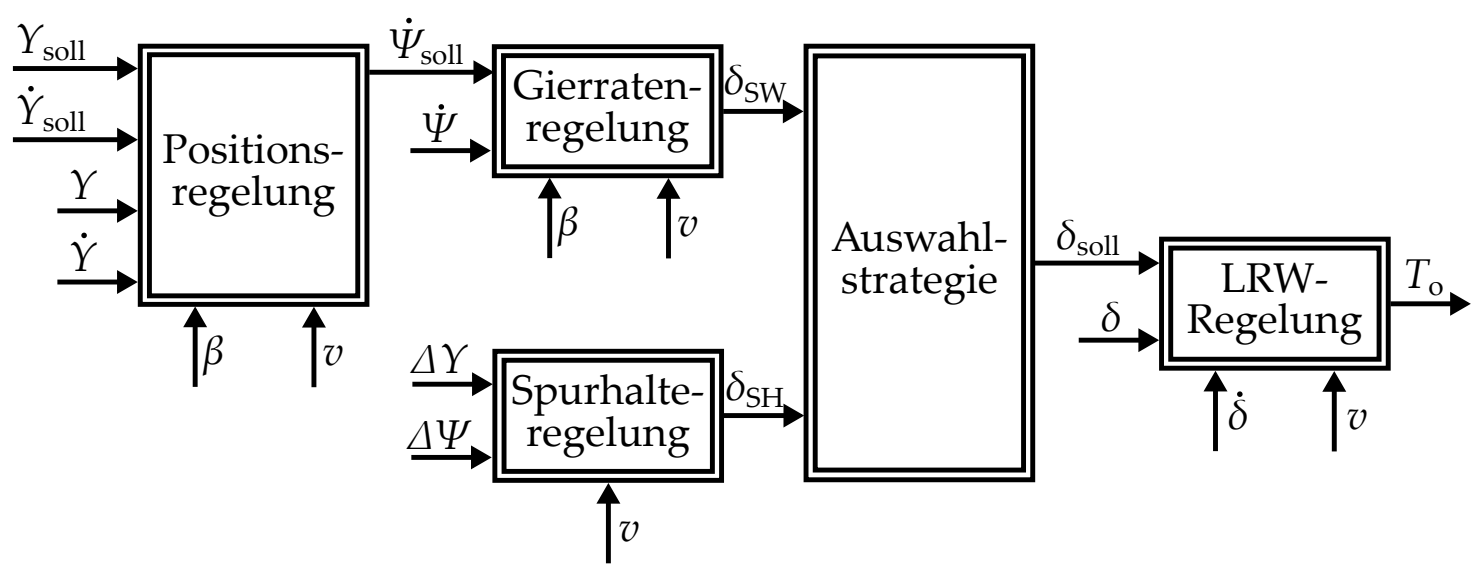

Abbildung 4.4.: Kaskadiertes Regelungskonzept zur Fahrzeugquerführung.

zwischen dem Spurwechselpfad und dem Spurhaltepfad. Diese Struktur entspricht dem Bahnplanungsverfahren, welches ebenfalls diese zwei Manöver unterscheidet. Zu Beginn des Manövers ist zunächst der Spurwechselpfad aktiv. Wenn das Spurwechselpolynom abgefahren wurde und der fahrdynamische Zustand als stabil klassifiziert wird, erfolgt die Umschaltung auf den Spurhalteregler. In der Regel werden die Spurmarkierungen der neuen Spur kurze Zeit später erkannt. Der Spurwechselpfad besteht aus einem überlagerten Positionsregler und einem unterlagerten Gierratenregler. Diese kaskadierte Reglerstruktur bietet sich aufgrund der folgenden Eigenschaften der Regelstrecke (Blockschaltbild in Abbildung 3.8) an:

- Die Ordnung der Regelstrecke ist hoch.

- Einige aber nicht alle Zustandsgrößen werden messtechnisch erfasst.

- Die Regelstrecke lässt sich in drei unabhängige Teile gliedern.

- Zwischen den Teilen kann jeweils eine Größe messtechnisch erfasst werden.

Für die Spurhalteregelung wird keine kaskadierte Struktur verwendet, da die Anforderungen an die Dynamik des Regelkreises niedriger sind. Alle Regleranteile sind geschwindigkeitsadaptiv (gesteuerter Adaption), da die Querdynamik stark von der Geschwindigkeit $v$ des Fahrzeugs abhängt (siehe [ [Kel+14c] $)$.

\section{Gierratenregelung}

Die Struktur der Gierratenregelung ist in Abbildung 4.5 dargestellt. Um die Dynamik des Regelkreises zu erhöhen, wird ein PD-Regler durch eine statische nichtlineare Vorsteuerung ergänzt. Die Kennlinie kann experimentell ermittelt werden. Hierzu wird bei konstanter Geschwindigkeit der Lenkradwinkel quasistationär erhöht, bis der 


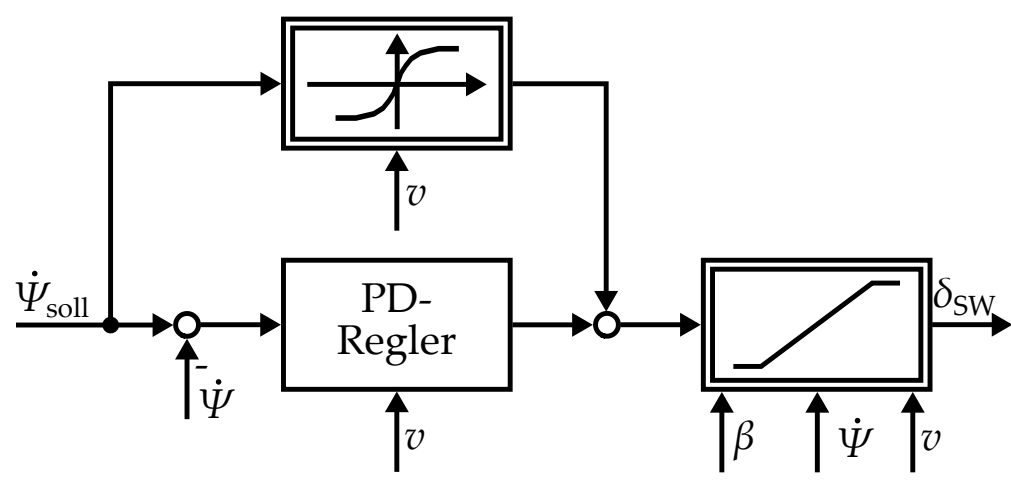

Abbildung 4.5.: Blockschaltbild der Gierratenregelung.

fahrdynamische Grenzbereich erreicht wird. Die Gierrate und der Lenkradwinkel werden gemessen. Es ergeben sich quasi unendlich viele stationäre Kreisfahrten, aus denen der Quotient $\delta / \dot{\Psi}$ mit geeigneter Diskretisierung ausgewertet wird. Alternativ kann das Experiment auch in der Simulation durchgeführt werden. Die addierten Ausgangsgrößen der Vorsteuerung und der Regelung unterliegen einer zustandsabhängigen Stellgrößenbeschränkung. Die beiden wesentlichen Nichtlinearitäten der Fahrdynamik werden Unter- und Übersteuern genannt. Zwischen diesen beiden extremen Zuständen verhält sich die Regelstrecke weitgehend linear. Durch die radselektiven Bremseingriffe des ESC, welches in jedem Neufahrzeug eingesetzt wird, werden die Zustände Unterund Übersteuern verhindert bzw. korrigiert. Die Wahl eines linearen Reglers ist daher als Grundlage einer Reglerstruktur zweckmäßig. Ein linearer Regler allein würde aber Eingriffe des ESC mehr als nötig provozieren. Als Beispiel dient eine Situation, in der eine sehr große Regelabweichung auftritt, welche jedoch eine kleine Änderung aufweist. Ein PD-Regler würde eine entsprechend große Stellgröße, in diesem Fall den Solllenkradwinkel, stellen. Dies führt bei Erregung aus der Ruhelage in der Regel zu Untersteuern. Der Lenkradwinkel würde zu stark ansteigen und das Fahrzeug kann dem Lenkeinschlag trotz unterstützender Eingriffe des ESC nicht folgen. Bei übersteuernden Fahrzuständen gelten ähnliche Zusammenhänge. Der lineare Regler kann das Fahrzeug nicht stabilisieren, insbesondere in Situationen, in denen sich Bahnfolge und Stabilisierung entgegenstehen. In diesem Fall wird die Stabilisierung durch das ESC erreicht. Trotzdem ist es von Vorteil, wenn weder übermäßig untersteuert noch übersteuert wird. Der PD-Regler wird daher durch eine zustandsabhängige Beschränkung ergänzt, um diese beiden Fahrzustände zu vermeiden. Um Untersteuern zu vermeiden, werden Ober- und Untergrenzen für den zulässigen Schräglaufwinkel definiert, die sich aus den Maximal- und Minimalstellen der Reifenkennlinien ergeben. Über die kinematischen Bindungen des Einspurmodells

$$
\begin{aligned}
& \delta_{\text {max }}=\alpha_{\mathrm{v}, \text { max }}+\arctan \left(\frac{l_{\mathrm{v}} \dot{\Psi}+v \sin \beta}{v \cos \beta}\right) \\
& \delta_{\text {min }}=\alpha_{\mathrm{v}, \text { min }}+\arctan \left(\frac{l_{\mathrm{v}} \dot{\Psi}+v \sin \beta}{v \cos \beta}\right)
\end{aligned}
$$

können Ober- und Untergrenzen für den Solllenkradwinkel unter der Voraussetzung angegeben werden, dass die Extrema der Reifenkennlinie bekannt sind. Um Übersteuern zu vermeiden, wird die Phasenebene des Einspurmodells betrachtet. Zunächst 


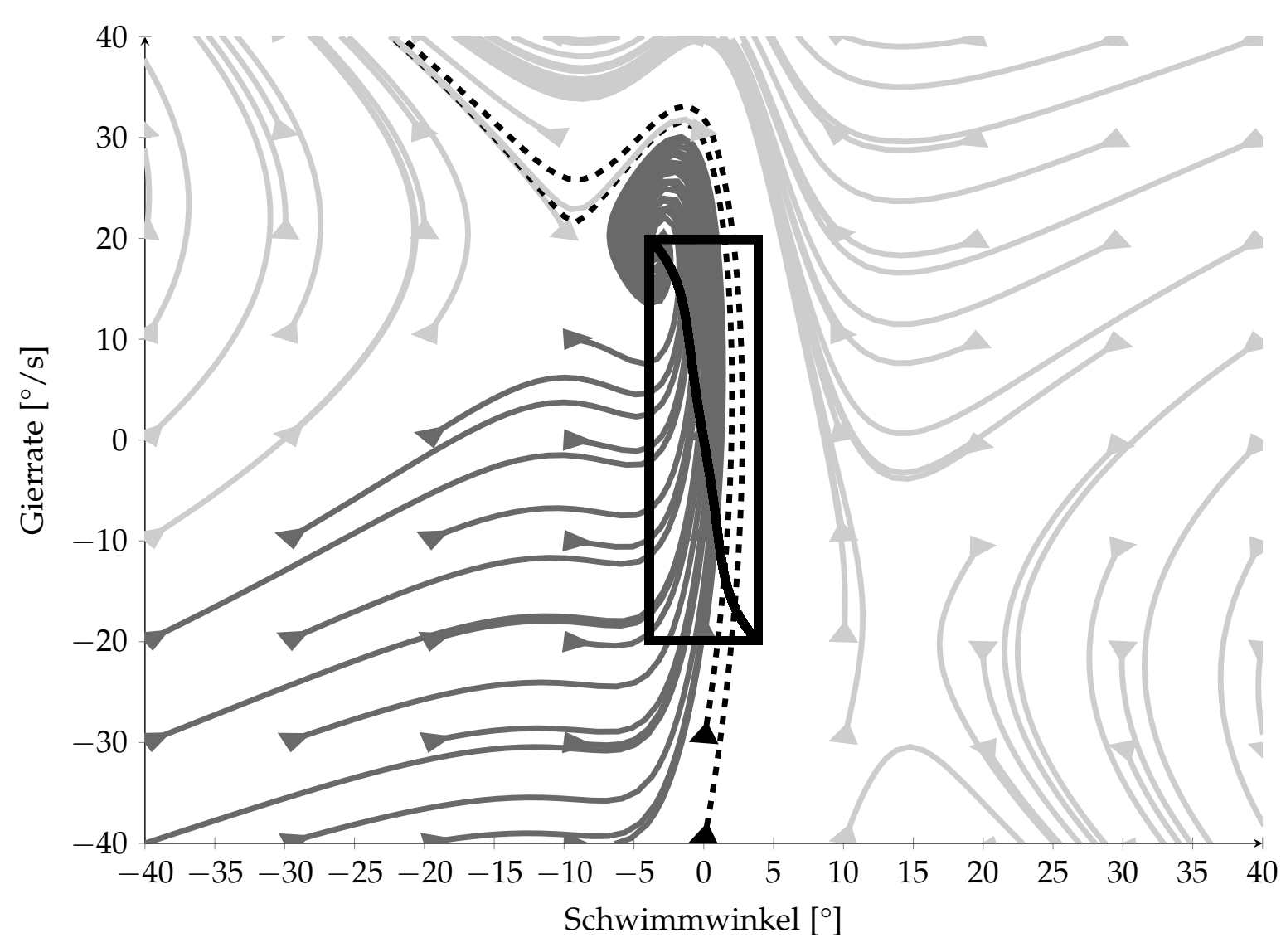

Abbildung 4.6.: Zustandstrajektorien in der Phasenebene bei der Geschwindigkeit $v=100 \mathrm{~km} / \mathrm{h}$ und dem Lenkradwinkel $\delta=85^{\circ}$ : Stabile (dunkelgrau nach Kriterium 4.3.4 bzw. gestrichelt nach Kriterium (4.3.5) ) und instabile (hellgrau) Trajektorien. Die schwarze Box markiert die Grenzwerte der stationären Kreisfahrt. Das Polynom der stationären Kreisfahrt wird durch die schwarze Linie innerhalb der Box dargestellt.

werden die stationären Kreisfahrtwerte $\beta_{\text {stat }}$ und $\dot{\Psi}_{\text {stat }}$ durch Erregung aus der Ruhelage mit einer Lenkwinkelrampe geringer Steigung bestimmt. Die Werte werden anschließend mit einem Polynom 5. Grades

$$
\hat{\beta}_{\text {stat }}=\sum_{i=0}^{5} c_{i} \hat{\Psi}_{\text {stat }}^{i}
$$

innerhalb der Maxima der stationären Kreisfahrtwerte $\beta_{\text {lim,stat }}$ und $\dot{\Psi}_{\text {lim,stat }}$ mittels Regression bestimmt. Anschließend werden die Zustandsdifferentialgleichungen für verschiedene Lenkradwinkel und Geschwindigkeiten von verschiedenen Startzuständen aus von $t=0$ bis $t=t_{\text {end }}$ gelöst. Einige Trajektorien konvergieren in die stationäre Kreisfahrt (stabil), andere streben ins Unendliche (instabil). In Abbildung 4.6 sind die Phasenebene und mehrere Trajektorien in einer Beispielsituation dargestellt. Im Folgenden werden Trajektorien stabil genannt, wenn sie entweder die Forderungen

$$
\left(\begin{array}{c}
\left|\dot{\beta}\left(t_{\text {end }}\right)\right| \\
\left|\ddot{\Psi}\left(t_{\text {end }}\right)\right|
\end{array}\right)<\left(\begin{array}{l}
\left|\dot{\beta}_{\text {lim,stat }}\right| \\
\left|\ddot{\Psi}_{\text {lim,stat }}\right|
\end{array}\right) \wedge\left(\begin{array}{c}
\left|\beta\left(t_{\text {end }}\right)\right| \\
\left|\dot{\Psi}\left(t_{\text {end }}\right)\right|
\end{array}\right)<\left(\begin{array}{l}
\left|\beta_{\text {lim,stat }}\right| \\
\left|\dot{\Psi}_{\text {lim,stat }}\right|
\end{array}\right)
$$


oder die Forderungen nach der Existenz des Schnittpunktes

$$
\beta(\dot{\Psi})=\hat{\beta}_{\text {stat }}\left(\hat{\dot{\Psi}}_{\text {stat }}\right) \text { für }\left(\begin{array}{c}
|\beta(t)| \\
|\dot{\Psi}(t)|
\end{array}\right)<\left(\begin{array}{l}
\left|\beta_{\text {lim,stat }}\right| \\
\left|\dot{\Psi}_{\text {lim,stat }}\right|
\end{array}\right)
$$

oder alle Forderungen erfüllen. Die Forderung (4.3.4) entspricht einer Trajektorie, welche in der stationären Kreisfahrt endet. Das heißt, dass der Anfangszustand durch den zugehörigen konstanten Lenkwinkel in einen stabilen Zustand überführt wird. Es gibt jedoch Startzustände, aus denen die Trajektorien zunächst in Richtung der stabilen Gleichgewichtszustände tendieren, das oben eingeführte Polynom 5. Grades $\hat{\beta}_{\text {stat }}$ (stationäre Kreisfahrt) schneiden und anschließend ins Unendliche streben. Diese Trajektorien werden $\mathrm{zu}$ den stabilen Trajektorien gezählt, da sie die Forderungen (4.3.5) erfüllen. Das ist deswegen sinnvoll, weil dadurch zunächst das Drehen des Fahrzeugs verhindert wird. Zudem besteht die Möglichkeit, den Lenkradwinkel im Bereich der stationären Kreisfahrt entsprechend anzupassen, sodass das Fahrzeug nicht zur Gegenseite übersteuert. Auf diese Weise werden Kennfelder berechnet, welche für jede Kombination aus dem Tupel $(v, \beta, \dot{\Psi}, \delta)$ einen booleschen Wert über die Stabilität (true oder false) zurückgeben. Daraus werden zwei Kennfelder berechnet, welche für das Tupel $(v, \beta, \dot{\Psi})$ den maximalen bzw. minimalen Lenkradwinkel ausgeben, der zu einer stabilen Trajektorie nach den obigen Forderungen (4.3.4) oder (4.3.5) führt.

\section{Positionsregelung}

In Abbildung 4.7 ist der überlagerte Positionsregler dargestellt. Er besteht aus der

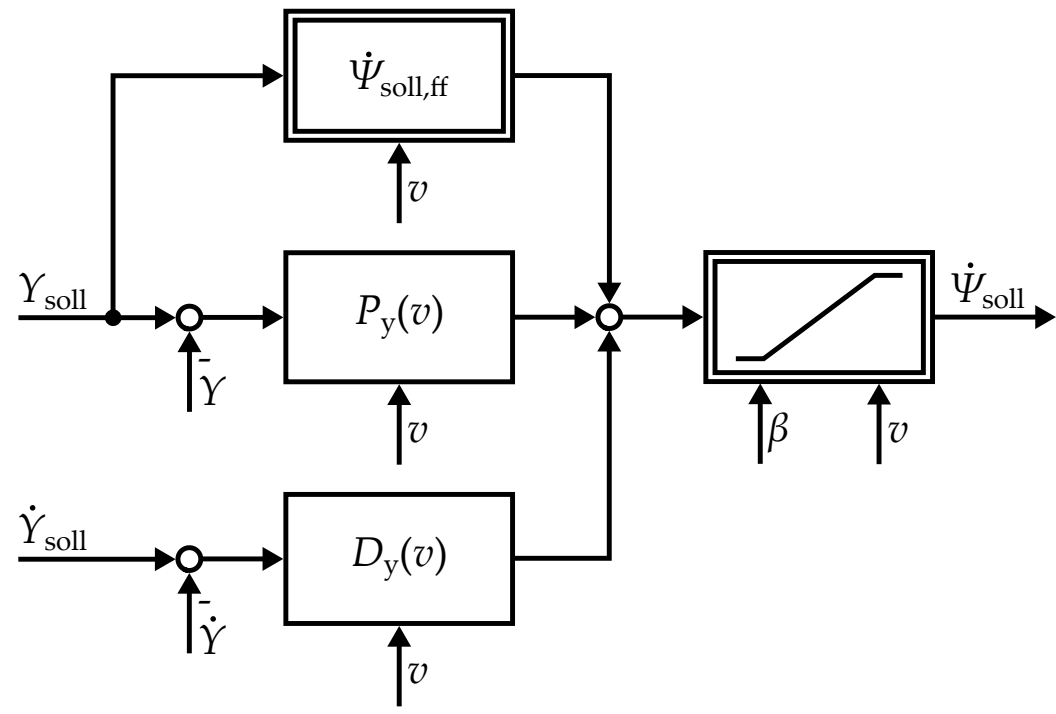

Abbildung 4.7.: Blockschaltbild der Positionsregelung.

nichtlinearen Vorsteuerung

$$
\dot{\Psi}_{\text {soll,ff }}=\frac{\mathrm{d}}{\mathrm{d} x}\left(\arctan \left(\frac{\mathrm{d} Y_{\text {soll }}}{\mathrm{d} x}\right)\right) v \cos \lambda
$$

und einem PD-Regler. Die Stellgrößenbeschränkung wird so gewählt, dass in Abhängigkeit des Schwimmwinkels nur diejenige Sollgierrate zugelassen wird, die stabilisierbar 
ist. Die Differentiation der Regelabweichung (D-Anteil des PD-Reglers) kann vermieden werden, da sich die Änderung der Sollgröße analytisch bestimmen lässt und die Änderung der Position des Ego-Fahrzeugs aus der Zustandsdifferentialgleichung (3.4.1) basierend auf beobachteten Größen ergibt.

\section{Spurhalteregelung}

Im Vergleich zum Notausweichen ist die Spurhaltung eine einfache Aufgabe. Nach einem Notausweichmanöver benötigt der Fahrer jedoch auch bei dieser vermeintlich leichten Aufgabe Unterstützung. Die Spurhalteregelung ist in Abbildung 4.8 dargestellt und besteht aus einem Positionsregler mit Totzone und quadratischer Kennlinie und einem P-Regler zur Regelung der Fahrzeugorientierung. Die Totzone erlaubt es dem

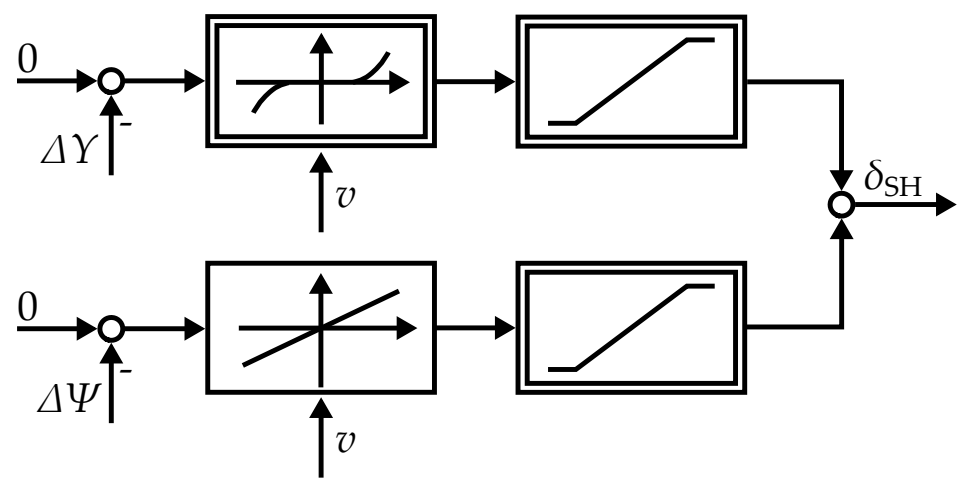

Abbildung 4.8.: Blockschaltbild der Spurhalteregelung.

Fahrer, eine Fahrzeugposition außerhalb der Fahrbahnmitte zu wählen ohne durch das System korrigiert zu werden. Dies könnte eher als störend empfunden werden, und nur wenige Fahrer fahren exakt in der Mitte der Fahrbahn. Um ein Verlassen der Fahrspur zu verhindern, werden quadratische Kennlinien verwendet, welche bei Annäherung an die Fahrbahnmarkierung stärkere Eingriffe erzeugen als eine lineare Kennlinie. Soll zu Demonstrationszwecken das Manöver autonom, d.h. ohne Fahrereinfluss, durchgeführt werden, dann kann ohne Totzone und mit einer linearen Kennlinie geregelt werden, um keine stationäre Regelabweichung zuzulassen (siehe Ergebnisse in Abbildung 4.14).

\subsection{Fahrzeuglängsregelung}

Das Regelungskonzept ist für die Querführung des Fahrzeugs entwickelt worden. Es lässt sich aber aufgrund der gesteuerten Geschwindigkeitsadaption durch zusätzliche längsdynamische Eingriffe erweitern, sodass Ausweichmanöver mit kombiniertem Brems- und Lenkeingriff durchgeführt werden können. Die Querführung wird als primäre und damit dominante Regelung festgelegt. Die Längsregelung wird untergeordnet. Nach dem in Kapitel 3 eingeführten Massenpunktmodell ist die Gesamtbeschleunigung des Fahrzeugs näherungsweise auf den Kammschen Kreis für Beschleunigung begrenzt. Da die Querführung dominieren soll, wird zur Längsführung die 
Sollbeschleunigung mit

$$
a_{\mathrm{x}, \text { soll }}= \begin{cases}\sqrt{a_{\mathrm{max}}^{2}-a_{\mathrm{y}}^{2}} & \text { für } a_{\mathrm{y}}^{2}<a_{\max }^{2} \\ 0 & \text { sonst }\end{cases}
$$

angegeben. Durch diese Wahl wird das Kraftschlusspotential stets ausgenutzt. Bei der Gestaltung des Fahrerassistenzsystems wurde gefordert, dass der Fahrer das System zu jedem Zeitpunkt übersteuern kann. Durch die Limitierung der Lenkmomentenüberlagerung in Höhe und Änderungsrate wird dies sichergestellt. Durch die Entwicklung der Bremsassistenten ist bekannt, dass viele Fahrer nicht schnell genug den nötigen Bremsdruck aufbauen, wenn eine Notbremsung mit maximaler Verzögerung erforderlich ist. Der Notbremsassistent wertet die Pedalstellung aus und erkennt, dass eine Notsituation vorliegt. Der Bremsdruck wird anschließend durch das System erhöht. Für die Unterstützung des Fahrers bei einem Bremsausweichmanöver wird eine ähnliche Strategie gewählt. Wenn der Fahrer bei der Betätigung des Bremspedals einen Schwellwert überschreitet, greift der ESA ein und erhöht die Verzögerung nach Gleichung (4.4.1). Der Fahrer kann diesen Eingriff in beide Richtungen überstimmen. Zum einen kann er jederzeit stärker Bremsen als der ESA dies tun würde und zum anderen kann er durch das Lösen des Bremspedals den Eingriff des System abschalten. Der Bremseingriff ist damit vom Fahrer gesteuert. Das Assistenzsystem kann aufgrund der lückenhaften Umfelderfassung nicht in allen Situationen eine geeignete Entscheidung treffen und ist daher auf die Steuerung durch den Fahrer angewiesen. Zum Beispiel ist bei sehr schnellem rückwärtigem Verkehr ein kombiniertes Bremsausweichmanöver unter Umständen nachteilig gegenüber einem reinen Lenkmanöver. Die Entscheidung über die Richtung und die Art des Manövers muss daher vom Fahrer getroffen werden, welcher den Verkehrsraum überwachen muss.

\subsection{Ergebnisse}

Die folgenden Abbildungen zeigen Messergebnisse von Notausweichmanövern aus dem Fahrsimulator. Zunächst werden in der Abbildung 4.9 reine Lenkmanöver mit verschiedenen Startgeschwindigkeiten $\left(v_{\text {init }}=55,85,115\right.$ und $\left.145 \mathrm{~km} / \mathrm{h}\right)$ dargestellt. Die Geschwindigkeiten sind so gewählt, dass sie zwischen den Stützstellen der Kennfeldregler zur gesteuerten Geschwindigkeitsadaption liegen. Es ist zu erkennen, dass der Sollbahn ausreichend genau gefolgt wird, um bei jedem der gezeigten Manöver eine Kollision zu vermeiden. Das Manöver mit $v_{\text {init }}=85 \mathrm{~km} / \mathrm{h}$ zeigt eine sehr gute Realisierung des Sollverlaufs. Bei einer initialen Geschwindigkeit von $v_{\text {init }}=55 \mathrm{~km} / \mathrm{h}$ treten die größten Abweichungen auf. Die bei geringen Geschwindigkeiten erforderlichen großen Lenkradwinkel lassen sich mit dem Lenkaktuator nicht hinreichend schnell realisieren, sodass auch die Genauigkeit der Positionsregelung begrenzt ist. Je höher die Geschwindkeit ist, desto ausgeprägter zeigt sich ein überschwingendes Verhalten beim Einregeln des rechtsgekrümmten Teils der Ausweichbahn. Die maximalen Abweichungen von der Sollbahn werden in Tabelle 4.1 zusammengefasst. In Abbildung 4.10 wird ein kombiniertes Brems- und Lenkmanöver gezeigt, welches autonom durchgeführt wird. 

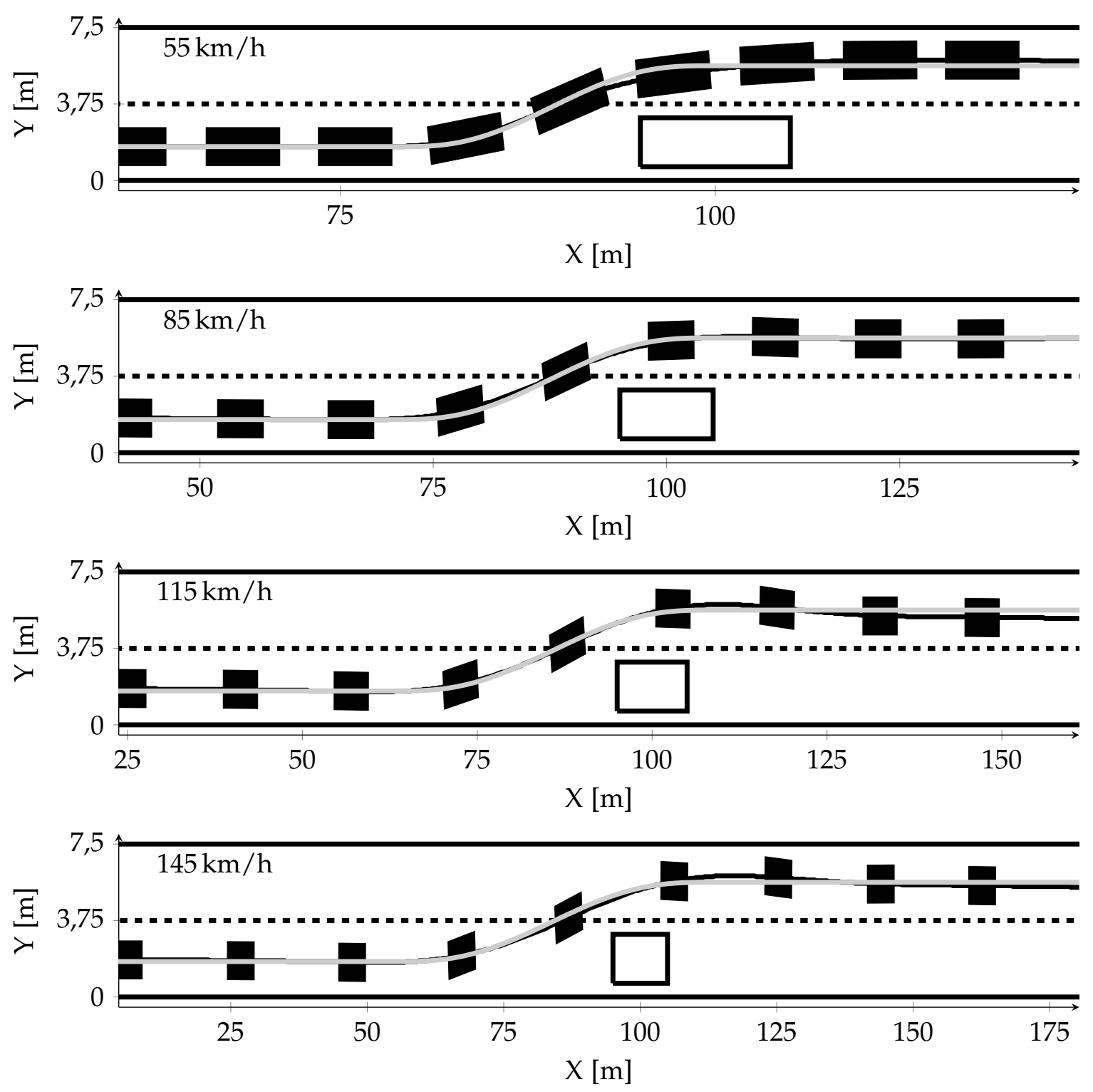

Abbildung 4.9.: Position des Ego-Fahrzeugs zu verschiedenen Zeitpunkten (schwarze Linie durch die ausgefüllten Vierecke, welche in Abständen von 0,5s dargestellt sind) bei autonomen Ausweichmanövern durch Lenkeingriff bei den initialen Geschwindigkeiten $v_{\text {init }}=55,85,115$ und $145 \mathrm{~km} / \mathrm{h}$. Die hellgraue Linie zeigt die Sollbahn an. Das Hindernis und die Fahrbahnbegrenzung sind durch schwarze Linien gekennzeichnet. Die Spurmittenmarkierung ist gestrichelt dargestellt.

Das Beschleunigungspotential des Fahrzeugs wird vollkommen ausgenutzt. Für kurze Zeit werden Beschleunigungen außerhalb des Kammschen Kreises erreicht, was im idealisierten Modell nach Kamm begründet ist. Die Sollbahn wird mit hoher Genauigkeit realisiert, sodass keine Kollisionen mit dem Hindernisfahrzeug oder der Straßenbegrenzung auftreten. Die Stabilität wird während des Manövers nicht gefährdet, wie die Phasenebene zeigt, und die unterlagerten Regler realisieren die Sollverläufe des Lenkradwinkels und der Gierrate mit hoher Genauigkeit. Das Ego-Fahrzeug kommt neben dem Hindernisfahrzeug zum Stehen. In der in Kapitel1 beschriebenen Situation des Stauendes ist dies ein erstrebenswertes Ergebnis. Wie bereits erwähnt, gilt dies jedoch nicht für jede Situation. In Abbildung 4.11 wird ein assistiertes Manöver gezeigt. 

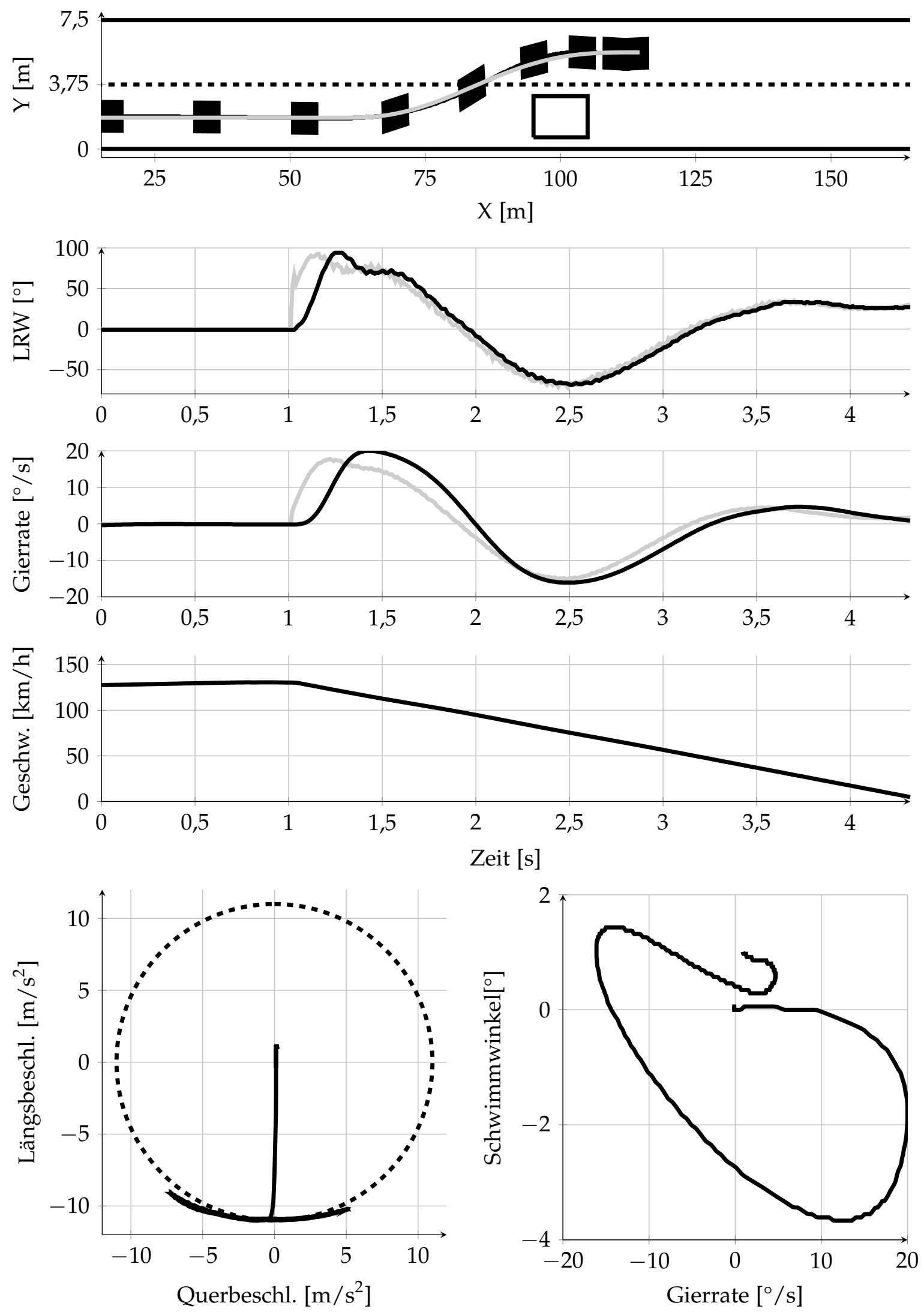

Abbildung 4.10.: Autonomes Ausweichmanöver mit kombiniertem Lenk- und Bremseingriff bei einer initialen Geschwindigkeit $v_{\text {init }}=130 \mathrm{~km} / \mathrm{h}$. Sollgrößen sind durch graue, Istgrößen durch schwarze Linien dargestellt. Das Ego-Fahrzeug ist in Abständen von 0,5s abgebildet. 

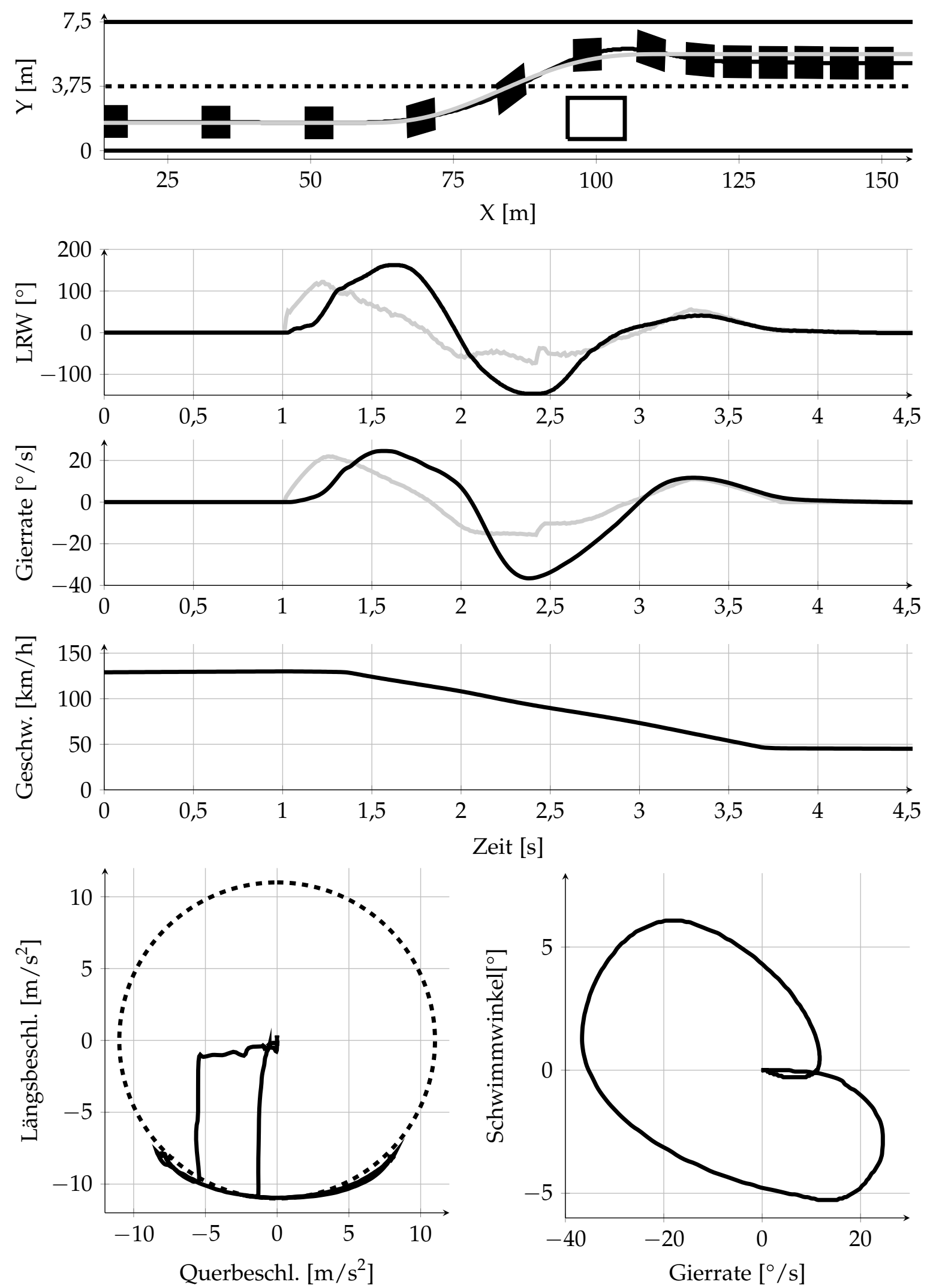

Abbildung 4.11.: Assistiertes Ausweichmanöver mit kombiniertem Lenk- und fahrergesteuertem Bremseingriff bei einer initialen Geschwindigkeit $v_{\text {init }}=130 \mathrm{~km} / \mathrm{h}$. Sollgrößen sind durch graue, Istgrößen durch schwarze Linien dargestellt. Das Ego-Fahrzeug ist in Abständen von $0,5 \mathrm{~s}$ abgebildet. 
In diesem Fall wird die fahrergesteuerte Längsführung eingesetzt. Es ist zu erkennen, dass die Sollgrößen weniger genau eingeregelt werden. Die Genauigkeit bleibt aber ausreichend, um die Kollision zu vermeiden. Aufgrund der weniger geeigneten Lenkbewegungen des Fahrers erreicht der Schwimmwinkel höhere Werte als $5^{\circ}$. Die bei vielen Fahrern zu beobachtenden Schleuderbewegungen (siehe Kapitel 2) werden jedoch vermieden, obwohl kein ESC modelliert ist. Es wird nicht bis in den Stillstand gebremst, da nach dem Ausweichmanöver eine normale Fahrsituation vorherrscht, der Fahrer nicht mehr bremst und dementsprechend kein weiterer längsdynamischer Eingriff erfolgt. Nach dem Manöver wird das Fahrzeug dauerhaft neben der Spurmitte geführt. Dennoch greift das System nicht ein, da die Abweichung innerhalb der Totzone des Spurhaltereglers liegt. Der Fahrer wird nicht unnötig gestört, zumal das Ego-Fahrzeug innerhalb der Spurmarkierungen geführt wird. Die stationären Abweichungen treten bei den autonomen Manövern ebenfalls auf. Die maximale Abweichung $\Delta_{\max }$ der tatsächlichen Bahn von der Sollbahn und die Stelle $X_{\Delta, \max }$, an welcher diese auftritt, ist für die zuvor beschriebenen sechs Manöver in Tabelle 4.1 zusammengefasst. Hierfür wurden die ersten zwei Sekunden nach Auslösung des Manövers betrachtet, da dieser Zeitraum für die Kollisionsvermeidung im Vergleich zu Abweichungen aus der Spurmitte nach dem eigentlichen Ausweichmanöver wichtiger ist.

Tabelle 4.1.: Maximale Abweichungen $\Delta_{\max }$ von der Sollbahn $Y_{\text {soll }}$ an der Stelle $X_{\Delta, \max }$.

\begin{tabular}{|c|c|c|c|}
\hline Manöver & $v_{\text {init }}[\mathrm{km} / \mathrm{h}]$ & $\Delta_{\max }[\mathrm{cm}]$ & $X_{\Delta_{\max }}[\mathrm{m}]$ \\
\hline Autonomes Lenkmanöver & 55 & 42,41 & 96,10 \\
\hline Autonomes Lenkmanöver & 85 & 20,81 & 79,62 \\
\hline Autonomes Lenkmanöver & 115 & 27,50 & 107,75 \\
\hline Autonomes Lenkmanöver & 145 & 31,50 & 113,71 \\
\hline Autonomes Lenk- und Bremsmanöver & 130 & 24,89 & 96,76 \\
\hline Assistiertes Lenk- und Bremsmanöver & 130 & 38,66 & 102,84 \\
\hline
\end{tabular}

Zum einen ist festzustellen, dass die maximalen Abweichungen an unterschiedlichen Stellen auftreten können. Ferner bleiben die Regelabweichungen bei autonomen Manövern im Bereich von ca. 20 bis $32 \mathrm{~cm}$ mit Ausnahme des Manövers mit $55 \mathrm{~km} / \mathrm{h}$. In diesem Geschwindigkeitsbereich wirkt sich die nach oben begrenzte Lenkwinkelgeschwindigkeit des Aktors aus und verhindert ein besseres Ergebnis. Bei derart niedrigen Geschwindigkeiten ist in der Regel aber ohnehin eine Notbremsung vorzuziehen. Beim kombinierten Bremsausweichmanöver ist keine Abnahme der Regelungsgüte zu beobachten, was darauf schließen lässt, dass die gesteuerte Geschwindigkeitsadaption der PD-Regler zweckmäßig ist. Das hier gezeigte assistierte Manöver weist eine etwas schlechtere Regelungsgüte gegenüber den autonomen Manövern auf, was aufgrund des Fahrereinflusses zu erwarten war. Insgesamt ist festzustellen, dass die Präzision der Assistenz über der von Durchschnittsfahrern liegt. In der Probandenstudie in Kapitel 2 hat sich gezeigt, dass die Fahrzeugführung durch viele Fahrer derart unpräzise ist, dass Abweichungen von mehreren Metern zur Sollbahn zu beobachten sind und dadurch Kollisionen auftreten. Die Assistenzfunktion ist somit geeignet, das Fahrverhalten zu verbessern. 


\subsection{Verifikation im Versuchsfahrzeug}

Die folgende Abbildung 4.12 zeigt Messergebnisse aus dem Versuchsfahrzeug bei Ausweichmanövern mit ca. 60 bzw. 100 km/h Startgeschwindigkeit.

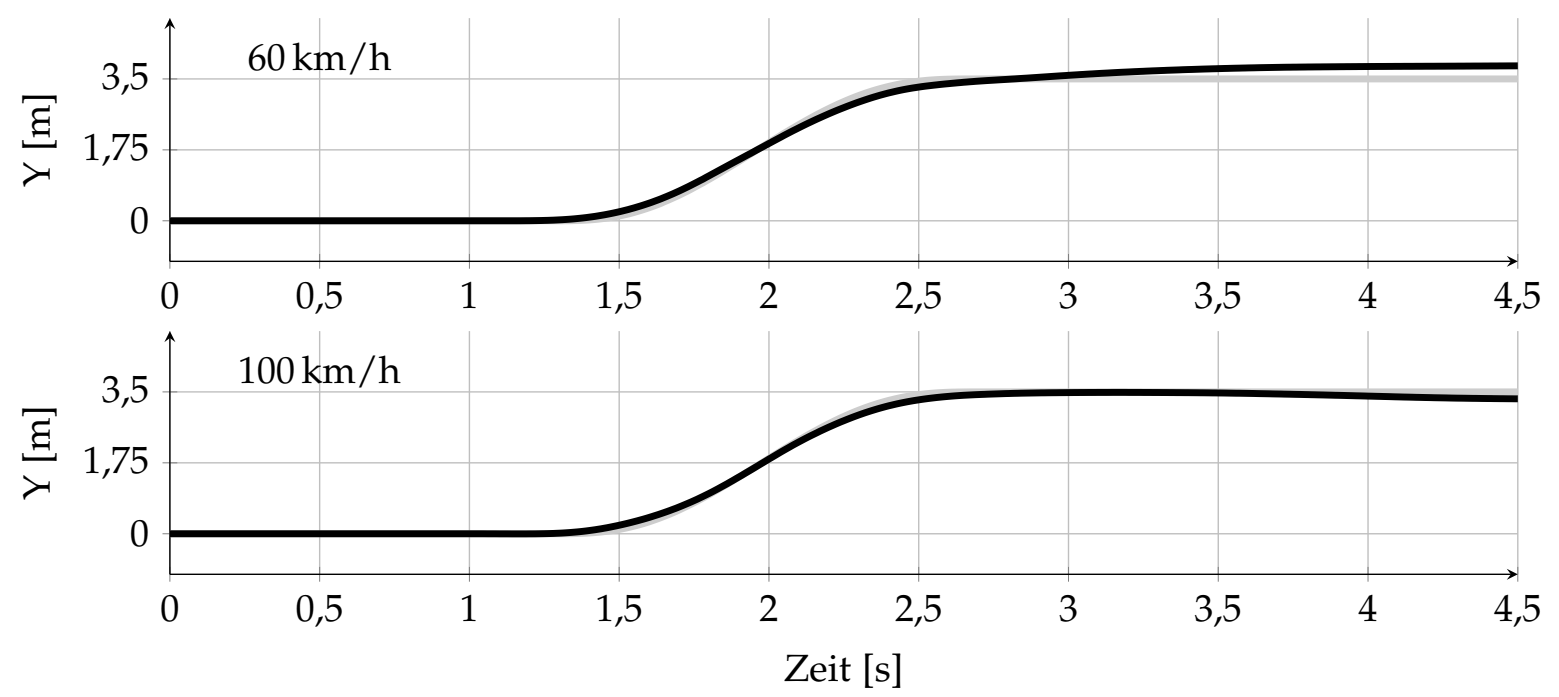

Abbildung 4.12.: Messergebnisse autonomer Ausweichmanöver aus dem Versuchsfahrzeug: Oben bei $60 \mathrm{~km} / \mathrm{h}$, unten bei $100 \mathrm{~km} / \mathrm{h}$. Die Position des Fahrzeugs ist eine berechnete Größe. Sollgrößen sind in grau, Istgrößen in schwarz dargestellt.

Es ist zu erkennen, dass die Realisierung der Sollbahn mit hoher Genauigkeit erfolgt, welche mit den Ergebnissen aus dem Fahrsimulator vergleichbar sind. Auch hier nimmt die Abweichung wegen der Totzone der Spurhalteregelung mit der Zeit zu. Die maximale Abweichung von der Sollbahn beträgt auf dem Intervall $0<t<2 \mathrm{~s}$ für das Manöver mit ca. $60 \mathrm{~km} / \mathrm{h} \Delta_{\max }=16,4 \mathrm{~cm}$ bei $t=1,34 \mathrm{~s}$ und für das Manöver mit ca. $100 \mathrm{~km} / \mathrm{h} \Delta_{\max }=13,5 \mathrm{~cm}$ bei $t=1,44 \mathrm{~s}$. Da das reale Versuchsfahrzeug bei jedem Manöver unterschiedliche Ergebnisse liefert, wird in Abbildung 4.13 die Streuung bei der Realisierung der Sollbahn gezeigt.

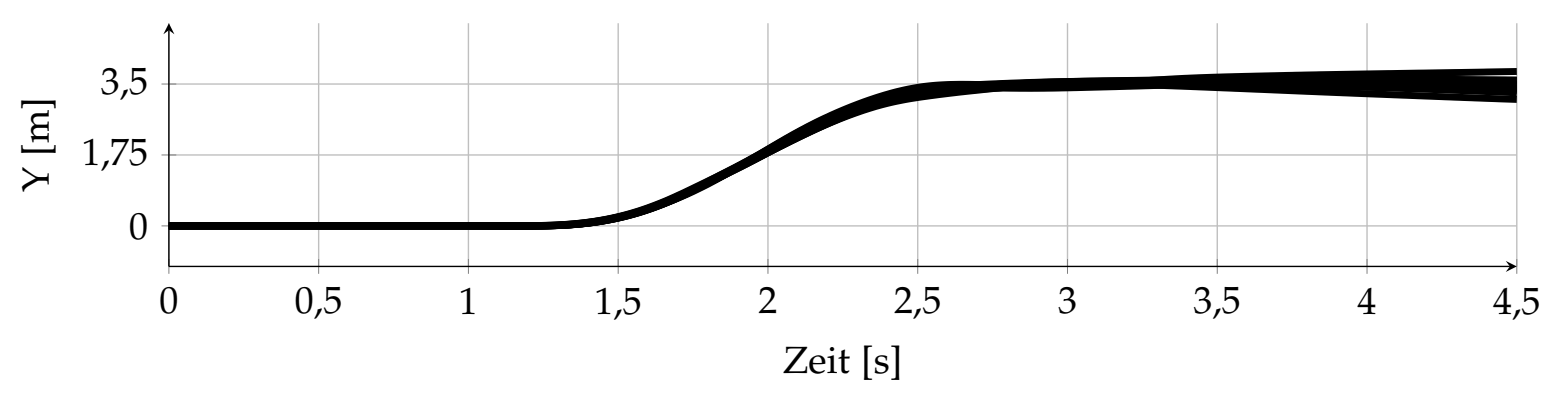

Abbildung 4.13.: Unsicherheit der Bahnfolgeregelung bei autonomen Ausweichmanövern.

Das Manöver wurde unter gleichen Bedingungen (gleicher Reibwert des Rad-StraßeKontaktes, gleiche Temperatur, gleiches Messgelände, gleiche Beladung) gefahren und es zeigt sich, dass die Abweichung zu Beginn des Manövers gering ist. Dies bedeutet, dass das vordergründige Ziel der Kollisionsvermeidung stets erreicht wird. Bis zum 
Zeitpunkt $t=2 \mathrm{~s}$ beträgt die maximale Abweichung von der Sollbahn über alle gezeigten realisierten Bahnen $25 \mathrm{~cm}$. Nach dem Spurwechsel treten größere Abweichungen auf, was dem Konzept der Spurhalteregelung geschuldet ist. Durch die Totzone werden kleinere Abweichungen nicht ausgeregelt. In der Abbildung 4.12 ist die Tendenz einer über der Zeit größer werdenden Abweichung vom Sollwert ebenfalls zu erkennen. Wird die Totzone nicht verwendet, verschwindet die stationäre Regelabweichung von der Sollbahn, wie in der Abbildung 4.14 bei einem exemplarischen Manöver zu sehen ist.
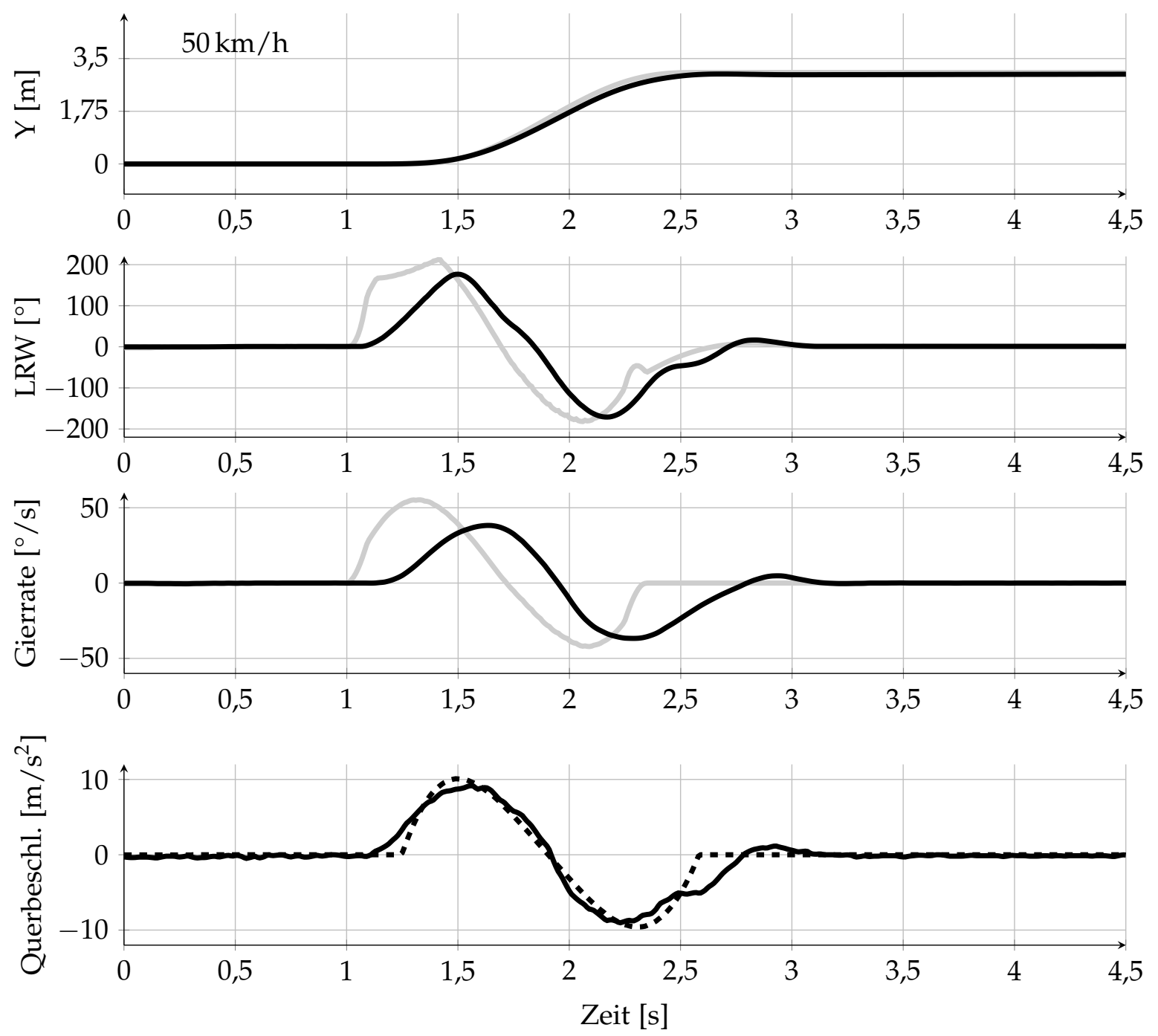

Abbildung 4.14.: Messergebnis eines autonomen Ausweichmanövers aus dem Versuchsfahrzeug bei ca. $50 \mathrm{~km} / \mathrm{h}$. Sollwerte sind in grau, Istwerte in schwarz dargestellt. Die gestrichelte Linie zeigt die zur Sollbahn gehörende Querbeschleunigung als Orientierungshilfe.

Die unterlagerten Regler weisen im Vergleich zu den Ergebnissen aus dem Fahrsimulator eine geringere Genauigkeit auf, da die Begrenzung des Lenkmomentes etwas restriktiver ist (siehe auch Kapitel 2). Die maximale Abweichung von der Sollbahn ist mit $\Delta_{\max }=20,28 \mathrm{~cm}$ bei $t=1,16 \mathrm{~s}$ mit den Ergebnissen aus dem Fahrsimulator vergleichbar. Die Geschwindigkeit (hier $50 \mathrm{~km} / \mathrm{h}$ ) verringert sich nur geringfügig während des Manövers, da es sich um ein reines Lenkmanöver handelt. Die Querbeschleunigung erreicht das Beschleunigungspotential des Fahrzeugs. 


\section{5}

\section{Online-Trajektorienoptimierungsverfahren}

Die Bahnberechnung in Kapitel 4 ist aufgrund der Beschränkung auf ein Polynom 5. Grades nur bedingt an unterschiedliche Verkehrssituationen anpassbar. Bei der Entwicklung des Online-Trajektorienoptimierungsverfahrens ist es deshalb das Ziel, dass sich die resultierende Trajektorie an die Verkehrssituation anpasst, sodass auch sehr komplexe Verkehrssituationen gelöst werden können. Aus der Literatur sind Verfahren bekannt, welche versuchen mit Regeln Eingriffsentscheidungen zu treffen [Ack+14]. Es stellt sich die Frage, ob diese Regeln (so umfangreich sie auch sein mögen) jede denkbare Situation lösen können. In [Ack+14] entscheidet ein Algorithmus zwischen Ausweich- und Bremsmanöver abhängig davon, ob auf der benachbarten Spur eine ausreichend große Lücke zur Verfügung steht. Dabei wird angenommen, dass alle Fahrzeuge jeweils die gesamte Spurbreite belegen. Wenn aber z.B. das Fahrzeug in der angrenzenden Spur näher zum Straßenrand hin fährt, so kann ausreichend Freiraum für ein Ausweichmanöver vorliegen, obwohl die Fahrbahn nach der Regel besetzt wäre. Als Folge dessen würde ein solcher regelbasierter Algorithmus eine ungeeignete Entscheidung treffen. Es kann argumentiert werden, dass unter diesen Umständen die Regel zu einfach ist und durch aufwändigere Regeln zu ersetzen ist. Es stellt sich jedoch dann die Frage, ob ein Regelsatz gefunden werden kann, welcher jede mögliche Situation löst. Man bedenke hierbei, dass auf einer mehrspurigen Autobahn (z.B. fünf Spuren pro Fahrtrichtung) mit mehreren Hindernissen sehr viele Optionen entstehen können. Aus Sicht des Autors ist der Versuch, mit immer mehr Regeln immer kompliziertere Verkehrssituationen zu lösen, nicht zielführend. Vielmehr ist ein Verfahren, welches Kollisionsvermeidungstrajektorien quasikontinuierlich an die jeweilige Verkehrssituation anpasst, zweckmäßig. Aus der mobilen Robotik ist das Timed Elastic Band (TEB) bekannt, welches in unstrukturierten Umgebungen mit mehreren (bewegten) Hindernissen optimale Trajektorien zu im Allgemeinen beweglichen Zielpunkten hin berechnet [Rös+12; Rös+13; Rös+15a]. Aus mehreren lokal optimalen Trajektorien wird die global optimale Lösung ausgewählt, sodass beliebig komplexe Situationen optimal gelöst werden. Die vorliegende Arbeit verwendet eine lokal optimale Trajektorie zur Kollisionsvermeidung. Die Erweiterung auf das globale Optimum sollte Gegenstand weiterer Forschungsprojekte sein und übersteigt den Rahmen dieser Arbeit. Die Architektur ist der des Bahnfolgeverfahrens insoweit ähnlich, als dass die Aufgaben Planung und Realisierung einer Trajektorie getrennt behandelt werden. Allerdings ist aufgrund der ständigen Neuberechnung der Trajektorie keine Positionsregelung erforderlich. Denn die Neuberechnung basiert auf der aktuellen Verkehrssituation und 
kinematischer Größen, was einer Rückführung entspricht und somit eine inhärente Positionsregelung durch das TEB erfolgt. Unterlagerte Fahrdynamikregler werden jedoch weiterhin benötigt. Die Architektur zeigt Abbildung 5.1. Anders als beim PFA ist die Trajektorienplanung abhängig vom Beschleunigungspotential des Fahrzeugs.

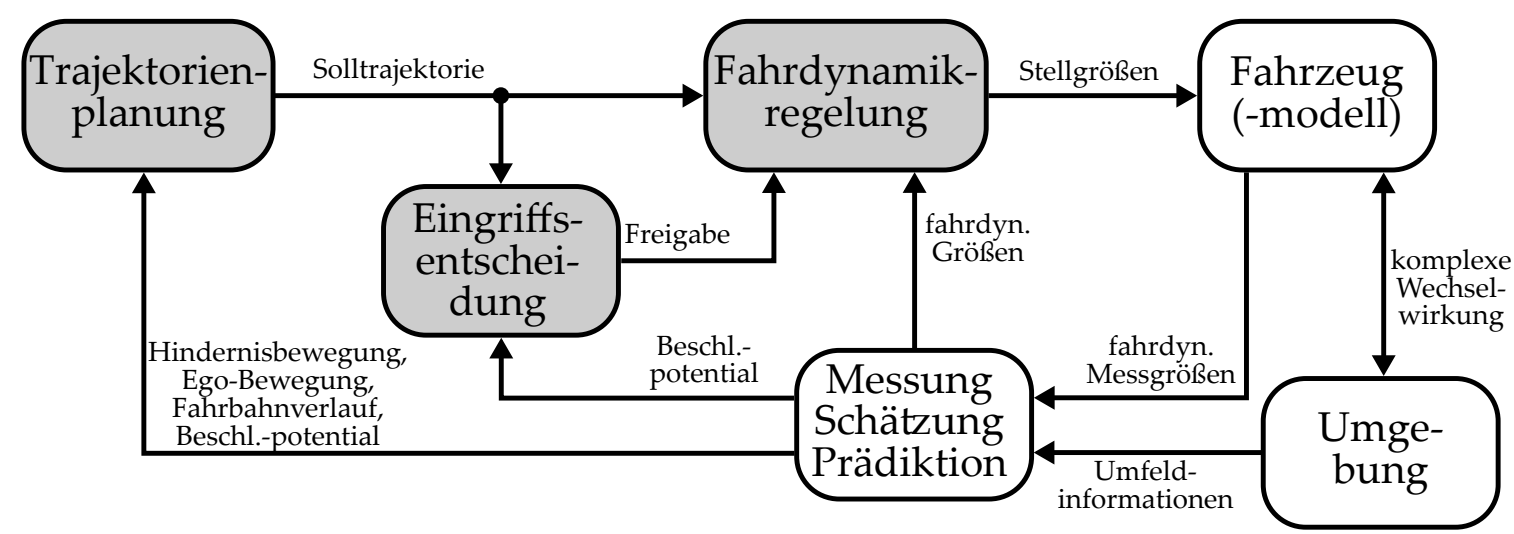

Abbildung 5.1.: Architektur des Online-Trajektorienoptimierungsverfahren.

\subsection{Beschreibung der Fahrzeugbewegung mit Timed Elastic Bands}

Das Timed Elastic Band ist definiert als

$$
T E B:=\left(Q_{\mathrm{TEB}}, \tau\right)
$$

und besteht aus der Menge

$$
Q_{\mathrm{TEB}}=\left\{P_{k}\right\}_{k=1,2, \ldots, n_{\mathrm{p}}}
$$

der Punkte

$$
P_{k}=\left(\begin{array}{l}
X_{k} \\
Y_{k}
\end{array}\right)
$$

und der Menge der zeitlichen Differenzen $\Delta T$ zwischen den Punkten $P_{k}$

$$
\tau=\left\{\Delta T_{k}\right\}_{k=1,2, \ldots, n_{\mathrm{p}}-1}
$$

Hinzu kommen die festen Anfangswerte $P_{0}=\left(\begin{array}{ll}X_{0} & Y_{0}\end{array}\right)^{\mathrm{T}}$ und $\Delta T_{0}$. Die Trajektorie wird also durch eine diskrete Menge von Punkten und Zeitdifferenzen, wie in Abbildung 5.2(a) dargestellt, zusammengesetzt. Die Formulierung 5.1.1) des TEB impliziert die Beschreibung der Fahrzeugbewegung mit einem Massenpunktmodell. Entlang der diskreten Trajektorie können entsprechend der Gleichungen in Kapitel 3 die kinematischen Größen aus Abbildung 5.2(a) berechnet werden. Die lokal optimale Trajektorie TEB* ergibt sich in der mobilen Robotik [Rös+12] aus der Lösung des Optimierungsproblems:

$$
T E B^{*}=\arg \min _{T E B} J_{\mathrm{TEB}} .
$$




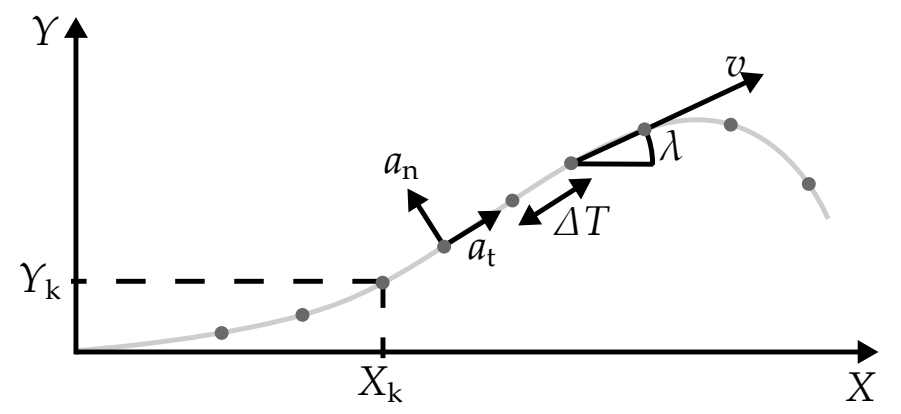

(a) Diskretisierung der Trajektorie und Veranschaulichung (b) der kinematischen Größen in natürlichen Koordinaten.

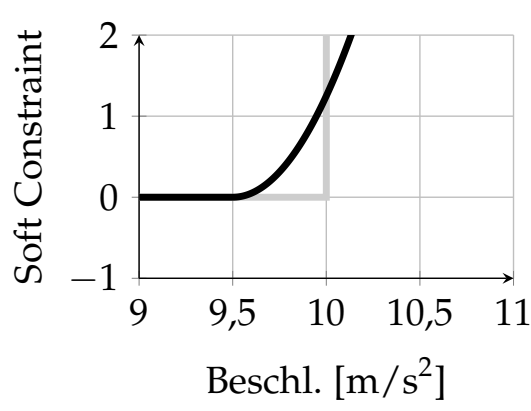

(b) Approximation der harten $\mathrm{Ne}-$ benbedingung (grau) durch die SoftConstraint Funktion (schwarz).

Abbildung 5.2.: Timed Elastic Band (a) und Soft-Constraint Funktion (b).

Das bedeutet, dass das gesamte Band als Optimierungsvariable aufgefasst wird. Durch die Minimierung der Zeitdifferenzen $\Delta T_{k}$ wird die schnellste Trajektorie zum Zielpunkt gefunden. Für die Kollisionsvermeidung im Straßenverkehr ist Zeitoptimalität jedoch weder erforderlich noch erstrebenswert. Die zeitlichen Differenzen $\Delta T_{i}$ werden daher konstant gewählt, sodass sich das Optimierungsproblem vereinfacht zu:

$$
T E B^{*}=\arg \min _{Q_{\mathrm{TEB}}} J_{\mathrm{TEB}} \text {. }
$$

Das Optimum ist definiert durch das Gütemaß

$$
J_{\mathrm{TEB}}=\sum_{i=1}^{n_{\gamma}} \gamma_{i} \boldsymbol{\Gamma}_{i}(\mathrm{TEB}),
$$

welches mehrere Ziele in der Form von quadratischen Kostentermen kombiniert. Der Kompromiss zwischen den Zielen $\Gamma_{i}(T E B)$ wird durch die Wahl der Gewichtungen $\gamma_{i}$ bestimmt. Zur Einhaltung von Grenzwerten und zur Vermeidung von Kollisionen werden Ungleichheitsnebenbedingungen

$$
\mathbf{g}(T E B)<0
$$

benötigt. Nichtlineare Least-Squares Probleme werden sowohl vom Dogleg als auch vom Levenberg-Marquardt Algorithmus [Hom15] effizient gelöst. Jedoch lassen sich mit beiden Algorithmen nur unbeschränkte Optimierungsprobleme lösen. Optimierungsalgorithmen, welche die Nebenbedingungen exakt einhalten, bedingen einen höheren Rechenaufwand. Statt der exakten Berücksichtigung, wird im Folgenden die Soft-Constraint Funktion

$$
f_{\mathrm{SC}}\left(z, z_{\mathrm{m}}, \epsilon\right)= \begin{cases}0 & \text { für } z \leq z_{\mathrm{m}}-\epsilon \\ \left(z-\left(z_{\mathrm{m}}-\epsilon\right)\right)^{2} & \text { für } z>z_{\mathrm{m}}-\epsilon\end{cases}
$$

verwendet und für jede Beschränkung in das Gütemaß integriert. Die Funktion ist beispielhaft in Abbildung 5.2(b) dargestellt. Die Beschleunigung soll in diesem Beispiel auf Werte $a<10 \mathrm{~m} / \mathrm{s}^{2}$ begrenzt werden. Die Sprungfunktion stellt die exakte 
Realisierung der Nebenbedingung dar und wird durch die weiche Nebenbedingung approximiert. Durch die geeignete Wahl der Gewichte ist sicherzustellen, dass die weichen Nebenbedingungen, falls sie verletzt werden, deutlich höhere Beiträge zum Gütemaß liefern als andere Kostenterme. Auf der einen Seite ist diese ungenaue Handhabung von Nebenbedingungen nachteilig, auf der anderen Seite ergibt sich durch diese Formulierung eine dünnbesetzte Struktur der Jakobi- und Hessematrix der Gütefunktion, was bei der Lösung des Optimierungsproblems ausgenutzt werden kann (siehe [Rös+12]).

\subsection{Entwurf des Gütemaßes zur Kollisionsvermeidung}

\subsubsection{Anforderungen an das Gütemaß}

Über das Gütemaß wird das Verhalten des Fahrzeugs festgelegt. Die Anforderungen an das Fahrzeugführungsverhalten sind

- Kollisionsfreiheit,

- Einhaltung der Beschleunigungsgrenzen,

- Fahrbarkeit bzw. Realisierbarkeit,

- minimale fahrdynamische Reaktionen und

- parallele Orientierung zur Fahrbahn.

Um kollisionsfreie Manöver zu realisieren, werden Hindernisse, wie andere Verkehrsteilnehmer und Fahrbahngrenzen, in einem Potentialfeld modelliert. Dabei erhalten Orte, an denen sich Hindernisse befinden bzw. befinden werden, ein hohes Potential und freie Verkehrsflächen das Nullpotential. Damit entspricht der Fahrkorridor der zusammenhängenden potentialfreien Fläche. Das Potentialfeld enthält keine Präferenz für einen bestimmten Ort innerhalb des Fahrkorridors. Im Sinne der Kollisionsvermeidung ist dies auch nicht erforderlich, da in einer Notsituation Verkehrsregeln wie z.B. das Rechtsfahrgebot keine Anwendung finden. Die Beschleunigungsgrenzen sind durch den Kammschen Kreis für Beschleunigungen gegeben und werden mit der Soft-Constraint Funktion berücksichtigt. Zusätzlich werden die Längs- und Querbeschleunigung bestraft, um die fahrdynamischen Reaktionen zu minimieren. Um die Realisierbarkeit/Fahrbarkeit zu erhöhen, wird zusätzlich der Ruck in beide Richtungen begrenzt und bestraft. Dies erhöht auch den Fahrkomfort in Situationen, die nicht unmittelbar gefährlich sind und entsprechend auch nicht das Beschleunigungspotential des Fahrzeugs ausschöpfen. Eine solche Situation liegt beispielsweise nach einem Notausweichmanöver vor, wenn das Fahrzeug noch eine gewisse Zeit geführt werden soll, bevor die Fahrzeugführung vollständig an den Fahrer zurückgegeben wird. In jeder Situation aber ist die Ausrichtung des Fahrzeugs parallel zur Fahrbahn erstrebenswert, was durch die Bestrafung des relativen Kurswinkels zur Fahrbahn im Gütemaß berücksichtigt wird. 


\subsubsection{Aufbau des Gütemaßes}

Im Folgenden wird das Gütemaß entsprechend der oben formulierten Anforderungen entworfen. Das Gütemaß lautet:

$$
\begin{aligned}
J_{\mathrm{TEB}} & =\sum_{k=1}^{n_{\mathrm{p}}} \gamma_{\mathrm{env}} \Gamma_{\text {env }, k}\left(P_{k}\right)+\cdots \\
& +\sum_{k=2}^{n_{\mathrm{p}}} \gamma_{\mathrm{a}, \mathrm{SC}}\left[f_{\mathrm{SC}}\left(a_{\mathrm{t}, k}, 0, \epsilon_{\mathrm{a}}\right)+f_{\mathrm{SC}}\left(a_{\mathrm{ges}, k}, \mathrm{~g}, \epsilon_{\mathrm{a}}\right)\right]+\cdots \\
& +\sum_{k=2}^{n_{\mathrm{p}}} \gamma_{\mathrm{a}, \mathrm{SC}}\left[f_{\mathrm{SC}}\left(a_{\mathrm{n}, k}, a_{\mathrm{n}, \lim }(v), \epsilon_{\mathrm{a}}\right)+f_{\mathrm{SC}}\left(-a_{\mathrm{n}, k}, a_{\mathrm{n}, \lim }(v), \epsilon_{\mathrm{a}}\right)\right]+\cdots \\
& +\sum_{k=3}^{n_{\mathrm{p}}} \gamma_{\mathrm{j}, \mathrm{SC}}\left[f_{\mathrm{SC}}\left(j_{\mathrm{t}, k}, j_{\mathrm{t}, l i m}, \epsilon_{\mathrm{j}, \mathrm{t}}\right)+f_{\mathrm{SC}}\left(-j_{\mathrm{t}, k}, j_{\mathrm{t}, l i m}, \epsilon_{\mathrm{j}, \mathrm{t}}\right)\right]+\cdots \\
& +\sum_{k=3}^{n_{\mathrm{p}}} \gamma_{\mathrm{j}, \mathrm{SC}}\left[f_{\mathrm{SC}}\left(j_{\mathrm{n}, k}, j_{\mathrm{n}, l i m}, \epsilon_{\mathrm{j}, \mathrm{y}}\right)+f_{\mathrm{SC}}\left(-j_{\mathrm{n}, k}, j_{\mathrm{n}, l i m}, \epsilon_{\mathrm{j}, \mathrm{n}}\right)\right]+\cdots \\
& +\sum_{k=1}^{n_{\mathrm{p}}} \gamma_{\lambda} \lambda_{k}^{2}+\sum_{k=2}^{n_{\mathrm{p}}} \gamma_{\mathrm{a}}\left[a_{\mathrm{t}, k}^{2}+a_{\mathrm{n}, k}^{2}\right]+\sum_{k=3}^{n_{\mathrm{p}}} \gamma_{\mathrm{j}}\left[j_{\mathrm{t}, k}^{2}+j_{\mathrm{n}, k}^{2}\right]
\end{aligned}
$$

Der Ruck in Längs- und Querrichtung wird hierbei durch

$$
j_{\mathrm{t}, k}=\frac{a_{\mathrm{t}, k}-a_{\mathrm{t}, k-1}}{\Delta T}, j_{\mathrm{n}, k}=\frac{a_{\mathrm{n}, k}-a_{\mathrm{n}, k-1}}{\Delta T}
$$

approximiert, sodass die aus der Drehung des Koordinatensystems resultierenden Ruckanteile (siehe Gleichungen (3.1.2) und (3.1.4)) nicht berücksichtigt werden. Die Approximation hängt stärker mit einer Stellgrößenänderung zusammen, welche in erster Linie durch diesen Gütemaßanteil minimiert werden sollen. Das im Gütemaß enthaltene Umfeldmodell (siehe [Thi15] und [Göt+16a]) besteht aus zwei Teilen:

$$
\Gamma_{\mathrm{env}, k}\left(P_{k}\right)=\Gamma_{1, k}\left(P_{k}\right)+\Gamma_{\mathrm{r}, k}\left(P_{k}\right) .
$$

Für die linke bzw. rechte Straßenseite gilt

$$
\Gamma_{1, k}\left(P_{k}\right)=f_{\mathrm{SC}}\left(Y_{k}, \min \left(Y_{\max }, f_{\mathrm{obs}}\left(X_{k}\right)\right), \epsilon_{\mathrm{Y}}\right)
$$

bzw.

$$
\Gamma_{\mathrm{r}, k}\left(P_{k}\right)=f_{\mathrm{SC}}\left(-Y_{k},-\max \left(Y_{\min }, f_{\mathrm{obs}}\left(X_{k}\right)\right), \epsilon_{\mathrm{Y}}\right)
$$

mit

$$
f_{\mathrm{obs}}\left(X_{k}\right)=\left\{\begin{array}{ll}
Y_{0}+\frac{Y_{\mathrm{end}, k}}{1+\mathrm{e}^{-\left(X_{k}-X_{\mathrm{obs}, k}-\Delta X\right)}} & X_{k}<X_{\mathrm{obs}, k} \\
Y_{0}+Y_{\mathrm{end}, k}-\frac{Y_{\mathrm{end}, k}}{1+\mathrm{e}^{-\left(X_{k}-X_{\mathrm{obs}, k}+\Delta X\right)}} & X_{k} \geq X_{\mathrm{obs}, k}
\end{array} .\right.
$$

Hierin ist

$$
\Delta X=-\frac{l_{\mathrm{obs}}}{2}-d_{\min , \mathrm{x}}
$$

In Abbildung 5.3 ist eine Skizze der Sigmoidalfunktion und der Variablen dargestellt. 


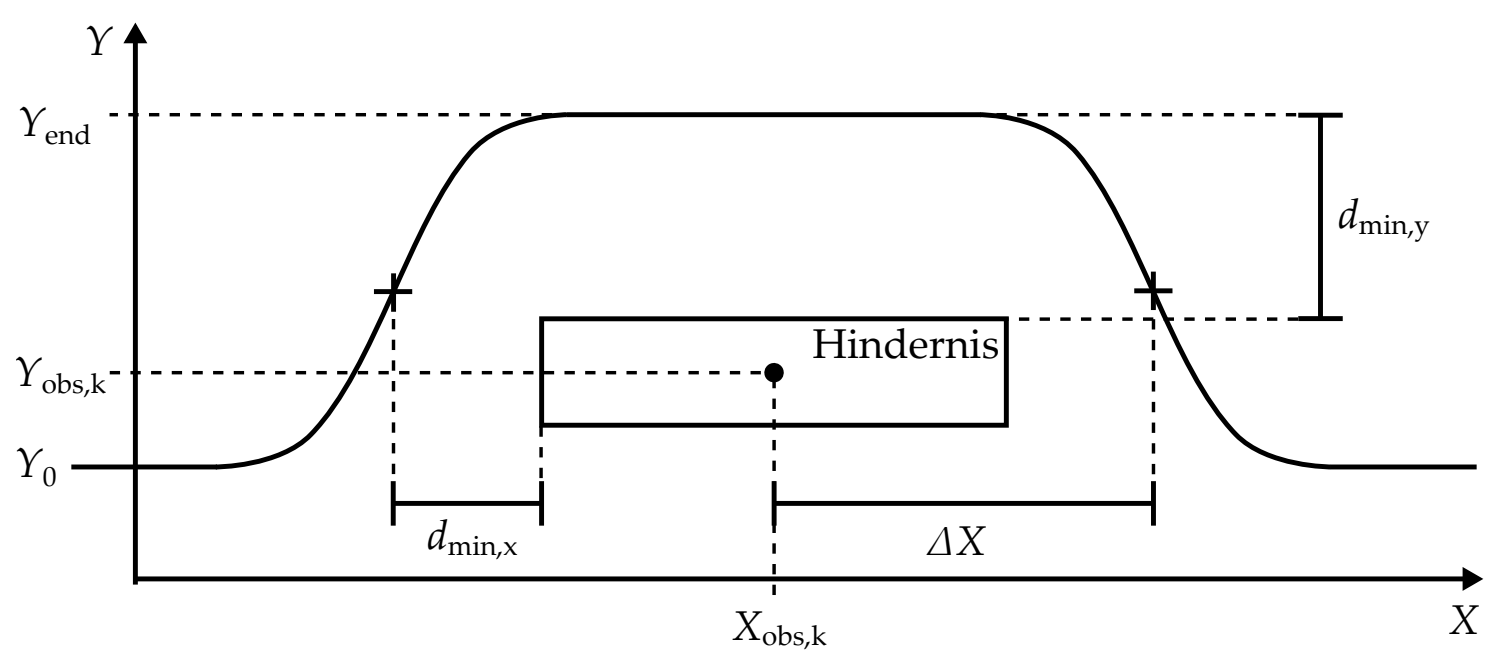

Abbildung 5.3.: Skizze zur Berechnung der Sigmoidalfunktion.

Wenn das Hindernis der rechten bzw. linken Spur zugeordnet wird gilt:

$$
Y_{0}=Y_{\min } \text { und } Y_{\mathrm{end}, k}=Y_{\mathrm{obs}, k}-Y_{\min }+\frac{b_{\mathrm{obs}}}{2}+d_{\mathrm{min}, \mathrm{y}}
$$

bzw.

$$
Y_{0}=Y_{\max } \text { und } Y_{\mathrm{end}, k}=Y_{\mathrm{obs}, k}-Y_{\max }-\frac{b_{\mathrm{obs}}}{2}-d_{\min , \mathrm{y}}
$$

Das Umfeldmodell besteht aus einer rechten Fahrbahnbegrenzung, einem potentialfreien befahrbaren Teil und der linken Begrenzung. Um ein Hindernis zu umfahren, wird abhängig davon auf welcher Fahrspur sich das Hindernis befindet entweder die linke oder rechte Begrenzung verschoben. Der Übergang zwischen der Fahrbahnbegrenzung auf eine Begrenzung durch ein Hindernis erfolgt durch eine Sigmoidalfunktion (siehe Gleichung (5.2.6)). In Abbildung 5.4(a) ist das Potentialfeld im Falle eines stehenden Hindernisses auf der rechten Fahrspur dargestellt. Das Potentialfeld ist, mit Ausnahme der Übergangsstellen der Sigmoidbögen zu den Geraden, stetig differenzierbar. Unter der Annahme, dass ein Fahrzeug auf der linken Spur nicht links bzw. ein Hindernis auf der rechten Fahrspur nicht rechts passiert werden kann, entspricht die lokal optimale Trajektorie der global optimalen Trajektorie. Der zweite Anteil im Gütemaß berücksichtigt den Kammschen Kreis, verhindert positive Längsbeschleunigungen (mit Bremseingriff nicht realisierbar) und beschränkt die Querbeschleunigung bei kleinen Geschwindigkeiten in dem Maße wie der maximale Lenkradwinkel es zulässt. In Abbildung 5.4(b) ist die im Simulationsexperiment bestimmte maximale stationäre Querbeschleunigung über der Geschwindigkeit aufgetragen. Der dritte Anteil des Gütemaßes beschränkt den maximalen Längs- und Querruck auf Werte, die durch die unterlagerten Fahrdynamikregler erreicht werden können. Die Maximalwerte sind ebenfalls im Simulationsexperiment bestimmt worden. Neben den Soft-Constraints werden weitere quadratische Kostenterme im Gütemaß berücksichtigt, welche das Fahrzeug bzw. die fahrdynamischen Reaktionen minimieren, indem der Kurswinkel, die Längsbeschleunigung, die Querbeschleunigung, der Längsruck und der Querruck im Gütemaß berücksichtigt werden. 

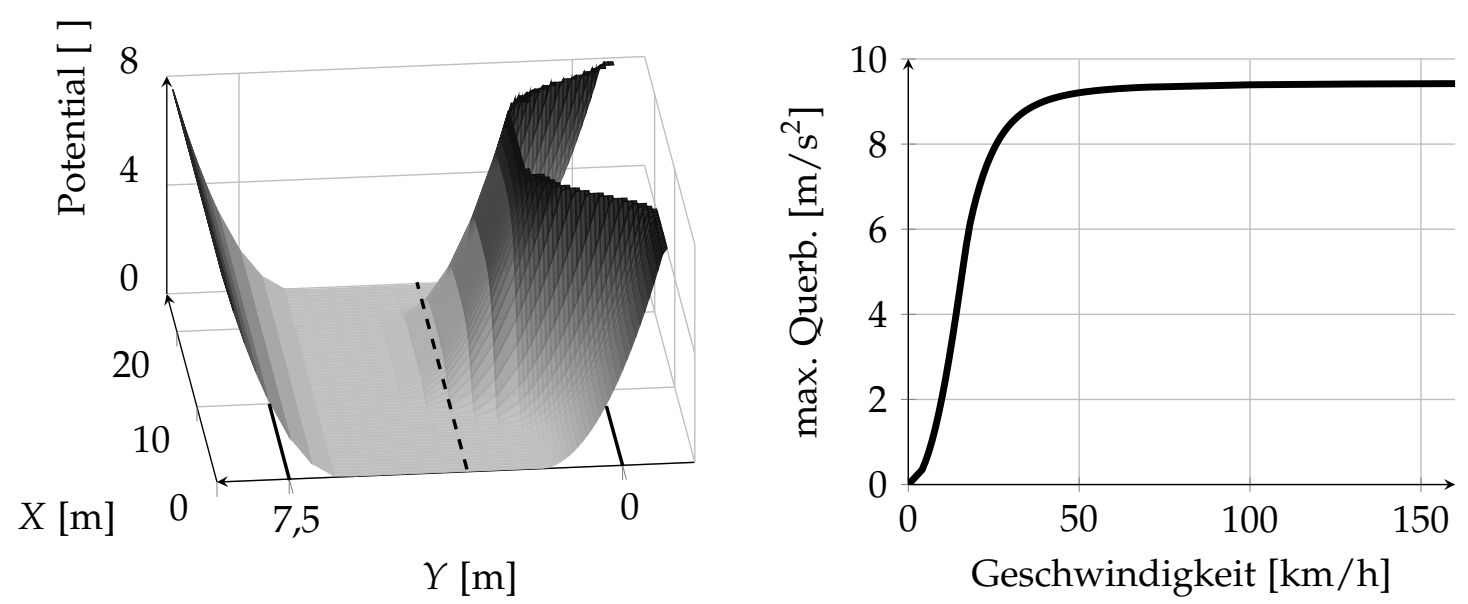

(a) Potentialfeld in einer Beispielsituation mit ei-(b) Maximale stationär erreichbare Querbeschleunem stehenden Hindernis auf der rechten Fahr-nigung des Fahrzeugmodells aufgetragen über spur. der Geschwindkeit.

Abbildung 5.4.: Umfeldmodell (a) und maximal erreichbare stationäre Querbeschleunigung (b).

\subsubsection{Zielkonflikte zwischen Gütemaßanteilen}

Die verschiedenen Anteile des Gütemaßes stehen sich teilweise entgegen. Im Folgenden werden zunächst die Konflikte und die Abhängigkeiten der Anteile voneinander erläutert. Beispielsweise wird der Zielkonflikt zwischen der Kollisionsvermeidung und der Minimierung der Gesamtbeschleunigung betrachtet. Diese Art von Zielkonflikt wird als eindeutig bezeichnet, da der Kollisionsvermeidung eindeutig eine höhere Bedeutung zukommt. Anders verhält es sich bei dem Zielkonflikt zwischen der Minimierung der Beschleunigung und des Ruckes. Es kann nicht allgemeingültig festgestellt werden, welchem Anteil eine höhere Bedeutung zuzumessen ist, da dies nicht zuletzt der individuellen Beurteilung der Passagiere unterliegt. Der Zielkonflikt wird als uneindeutig bezeichnet. In Abbildung 5.5 sind die verschiedenen Anteile gruppiert und ihre Beziehungen zueinander mit Pfeilen gekennzeichnet. Zwischen den Begrenzungen und Bestrafungen gibt es einen Zielkonflikt. Dieser ist jedoch durch die

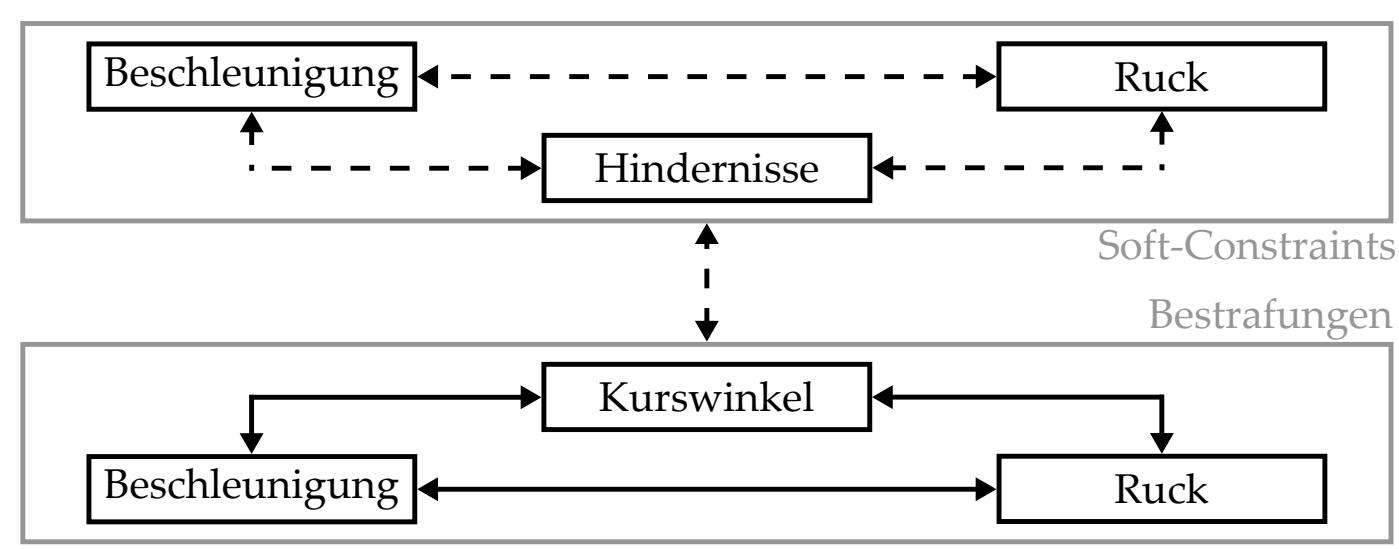

Abbildung 5.5.: Visualisierung der Gütemaßanteile und der auftretenden Zielkonflikte. Durchgezogene Pfeile symbolisieren uneindeutige, gestrichelte Pfeile eindeutige Zielkonflikte. 
manuelle Wahl lösbar, da die Soft-Constraints deutlich höher gewichtet werden als die Bestrafungen. Zwischen den einzelnen Soft-Constraints treten ebenfalls Zielkonflikte auf. Dies geschieht aber nur, wenn eine unvermeidbare Kollision bevorsteht. In einem solchen Fall ist aber die Lösung des Zielkonfliktes unerheblich und die Einleitung einer Vollverzögerung zur Reduktion der Unfallschere zweckmäßiger. Zwischen den Bestrafungen bestehen Zielkonflikte, deren Lösung von der Wahl der Gewichte bzw. von den Verhältnissen der Gewichte abhängt. Die Bestimmung der Gewichte stellt eine erneute Optimierungsaufgabe dar, welche numerisch oder heuristisch gelöst werden kann. Die manuelle Wahl der Gewichte ist zweckmäßig, da auch die Bewertung des Verhaltens der Fahrzeugführung subjektiv ist. Aufgrund der geringen Anzahl der Gewichte ist die heuristische Wahl überschaubar. Dies ist im Vergleich zu der Wahl der Gewichte beim modellprädiktiven Planungs- und Regelungsverfahren zu sehen, wo aufgrund des komplexeren Gütemaßes einige Gewichte nur bedingt manuell gewählt werden können.

\subsubsection{Kritikalitätsmaß und Eingriffsentscheidung}

Analog zum Bahnfolgeverfahren (Kapitel 4) bietet sich auch beim OTO die geplante Trajektorie zur Approximation der Kritikalität $K_{\text {pot }}$ basierend auf dem Nutzungsgrad der Bewegungspotentiale an (siehe Definition A.1.14). Neben der Querbeschleunigung kann hier aber auch die Längsbeschleunigung bzw. Gesamtbeschleunigung als Kritikalitätsindikator verwendet werden. Neben den Beschleunigungen können nur noch der Quer- und Längsruck als Maß für Kritikalität Anwendung finden. Die Ruckgrenzen von Fahrzeugen bzw. der vom Normalfahrer erreichte Ruck ist schwieriger zu bestimmen, während der Kammsche Kreis bzw. die Annäherung an den Kammschen Kreis ein universelles Maß für Kritikalität ist.

$$
K_{\mathrm{pot}}=\frac{a_{\mathrm{tot}}}{\mathrm{g}}
$$

Die Regelung wird daher freigegeben, sobald die maximale Gesamtbeschleunigung entlang der geplanten Trajektorie das Beschleunigungspotential des Fahrzeugs erreicht hat mit $K_{\text {pot }}=1$.

\subsection{Fahrdynamikregelung}

Anders als bei den in Kapitel 6 und 7 beschriebenen modellprädiktiven Verfahren enthält die Trajektorie beim OTO keine Stellgrößen, welche direkt als Sollgrößen an unterlagerte Regler weitergegeben werden können. Als Folge muss die Trajektorie durch geeignete Folgeregler realisiert werden. Da die Ausweichtrajektorie fortlaufend neu berechnet wird und sich damit der aktuellen Situation anpasst, können in einem ersten Schritt auch Steuerungen verwendet werden. In Abbildung 5.6 oben ist eine solche Steuerungsstruktur dargestellt. Die Struktur hat sich für normale Fahrsituationen als geeignet herausgestellt (siehe [Hom15] und [Hom+17]). Jedoch machen sich die Ungenauigkeiten der stationären Vorsteuerungen bei Notmanövern stärker bemerkbar. Der Versuch, die Genauigkeit der Steuerungen zu verbessern, ist nicht zielführend, da sich die Streckenparameter teilweise deutlich ändern können und die stationären 


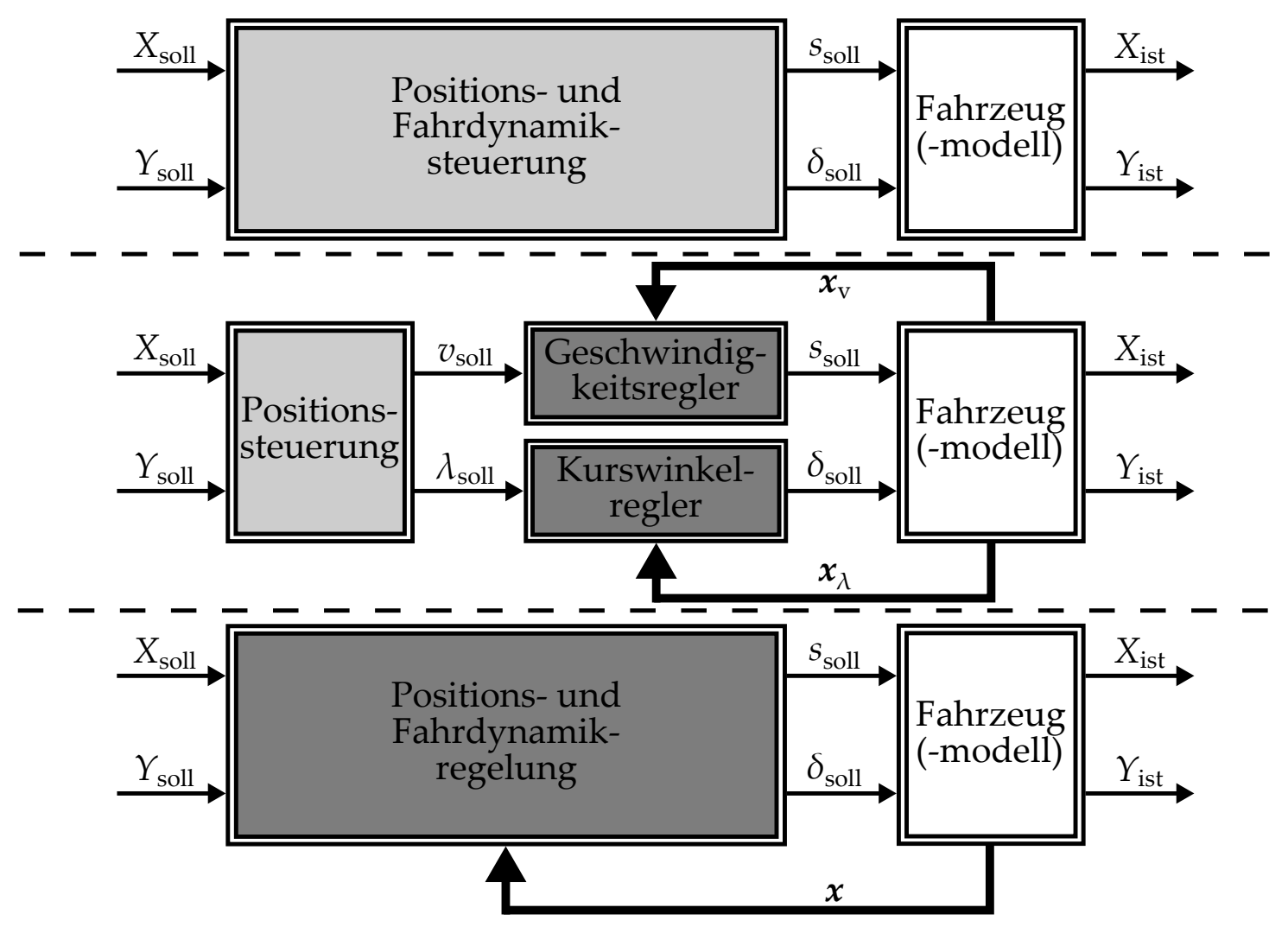

Abbildung 5.6.: Blockschaltbilder der Möglichkeiten, die geplante Trajektorie zu realisieren. Oben: Steuerung; mittig: Positionssteuerung und Fahrdynamikregelung; unten: Positions- und Fahrdynamikregelung.

Gleichungen der Dynamik der Regelstrecke nicht Rechnung tragen. Ähnlich dem Konzept beim Bahnfolgeverfahren scheint eine Trajektorienfolgeregelung sinnvoll, wie sie in Abbildung 5.6 unten dargestellt ist. Aufgrund der ständigen Neuberechnung der Trajektorie basierend auf der aktuellen Situation ist bereits eine Positionsregelung inhärent in der Trajektorienberechnung enthalten. Beim Bahnfolgeverfahren kommt ein schaltender Regler zum Einsatz, welcher einen Regelzweig für hohe Dynamik und einen für niedrige Dynamik enthält. Diese Wahl ist für das Konzept zweckmäßig, da entweder einer Spurwechseltrajektorie zu folgen oder eine Spur zu halten ist. Das OTO kann aber zwischen diesen beiden Extremen viele mitteldynamische Manöver planen. Das Design der Positionsregler wäre entsprechend aufwändig, da verschieden schnelle Dynamiken des geschlossenen Regelkreises benötigt werden. Aus der Literatur sind zudem Probleme bei der Aktualisierung der Trajektorie und der damit verbundenen sprungförmigen Änderungen der Sollgrößen bekannt (siehe [Hun+08]). Das bewährte Konzept der Regelung eines Vorausschaupunktes beschränkt sich auf einen Punkt, während beim OTO alle Punkte der Trajektorie zur Sollwertbildung beitragen. Darüber hinaus lassen sich aus dem TEB Steuerungsgleichungen ableiten, welche nicht von mit Unsicherheiten behafteten Parametern abhängen (siehe Gleichungen (3.1.1) und (3.1.3). In Abbildung 5.6 mittig ist die Fahrdynamikregelung im Blockschaltbild dargestellt. Die Bewegung eines Massenpunktes ist durch die Trajektorien des Kurswinkels $\lambda(t)$ und des Betrages des Geschwindigkeitsvektors $v(t)$ bestimmt. Diese beiden Größen 
bieten sich als Regelgrößen an, da die Geschwindigkeit im Wesentlichen durch den Bremseingriff, der Kurswinkel im Wesentlichen durch einen Lenkeingriff beeinflussbar ist. Letzteres trifft auch auf die Kursrate zu. Um Probleme mit der stationären Genauigkeit bei der Regelung zu vermeiden, eignet sich der Kurswinkel aufgrund der integralen Übertragungseigenschaften besser als Regelgröße. Alternativ ist die Trajektorie ebenfalls über die Längs- und Querbeschleunigungen definiert. Jedoch sind diese Größen keine Zustandsgrößen oder Linearkombinationen der Zustandsgrößen des Fahrdynamikmodells, was den Entwurf eines modellbasierten Reglers verhindert. Der Geschwindigkeits- und der Kurswinkelregler werden beide als lineare Zustandsregler nach Riccati ausgelegt. Hierzu wird die Regelstrecke zunächst linearisiert und die Kopplung zwischen der Längs- und Querdynamik vernachlässigt. Besonders im linearen Bereich der Fahrdynamik ist diese Annahme zulässig. Darüber hinaus zeigen die Ergebnisse in diesem Kapitel die Zweckmäßigkeit dieser Annahme. Die Geschwindigkeitsabhängigkeit der Querdynamik wird mittels gesteuerter Adaption berücksichtigt, was sich bereits bei dem Bahnfolgeregler in Kapitel 4 bewährt hat. Die Abbildung 5.7 zeigt die Sprungantworten der geschlossenen Regelkreise bei Anwendung der Regler auf die nichtlinearen Streckenmodelle. Bei kleineren Sollwertsprüngen
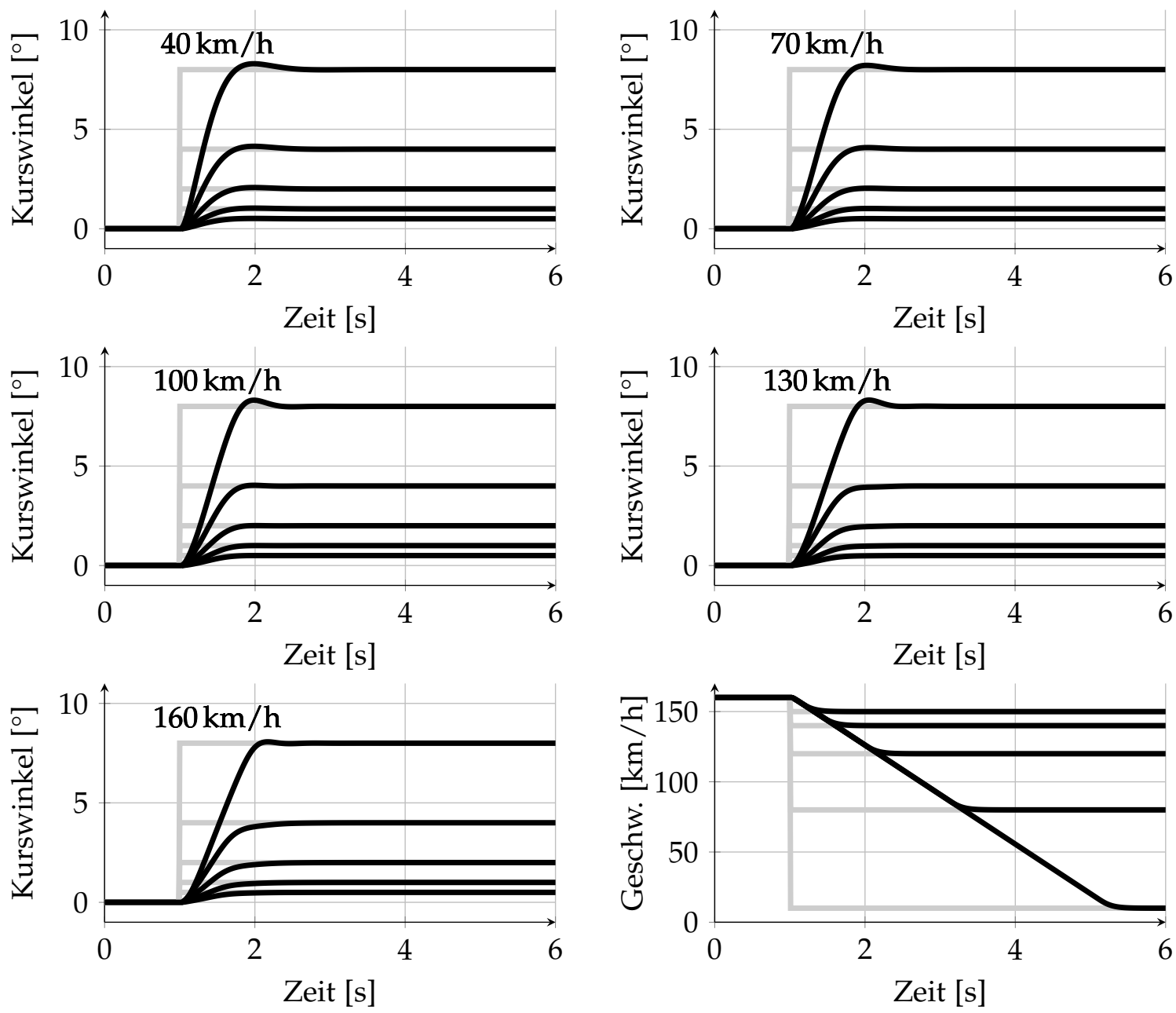

Abbildung 5.7.: Sprungantworten (schwarz) des Kurswinkelreglers für verschiedene Sollwerte (grau) und Geschwindigkeiten $v=40,70,100,130$ und $160 \mathrm{~km} / \mathrm{h}$ bzw. Sprungantworten (schwarz) des Geschwindigkeitsreglers (rechts unten) für verschiedene Sollwerte (grau). 
zeigt die Kurswinkelregelung kein Überschwingen, bei größeren Sprüngen ist moderates Überschwingen erkennbar. Die Geschwindigkeitsregelung zeigt unabhängig von der Sprunghöhe kein Überschwingen. Zu Beginn der Anregung bewegen sich alle Sprungantworten auf der gleichen Geraden, welche eine Steigung von $a_{\mathrm{x}} \approx \mathrm{g}$ aufweist. Ähnlich der Vorausschau bei der Positionsregelung wird ein in der Zukunft liegender Punkt der Geschwindigkeits- und der Kurswinkeltrajektorie als Sollgröße verwendet. Die Summenzeitkonstante des jeweiligen geschlossenen Regelkreises ist eine geeignete initiale Wahl für die Vorausschauzeit. Das Regelungsverhalten kann durch leichtes Erhöhen der Vorausschauzeit für höhere Geschwindigkeiten noch verbessert werden. Anders als bei der Verwendung von klassischen PID-Reglern sind die linearen Zustandsregler unabhängig von der Vorausschauzeit, da diese zwar Bestandteil der Ausgangsgleichung aber nicht der Zustandsdifferentialgleichungen der Regelstrecke sind. Dies erlaubt die getrennte Auslegung der Dynamik des Regelkreises und der Vorausschauzeit zur Kompensation des Schleppfehlers. Darüber hinaus können die Vorausschauzeiten für Quer- und Längszweig unterschiedlich gewählt werden, was aufgrund der Verkopplung der Positionsdynamik bei einer Trajektorienfolgeregelung so nicht möglich ist.

\subsection{Ergebnisse}

Das Führungsverhalten des OTO und der beiden nachfolgend beschriebenen Verfahren (Kapitel 6 und 7) werden in zwei unterschiedlichen Situationen und bei verschiedenen initialen Geschwindigkeiten untersucht. Das erste Szenario besteht aus vier stehenden Hindernissen, welche bei höheren Geschwindigkeiten einen Doppelspurwechsel und ein Anhaltemanöver erzwingen. Dieses Szenario wird mit drei verschiedenen Geschwindigkeiten simuliert, um die Entscheidungsfindung des Algorithmus zu zeigen. Das zweite Szenario besteht aus einem stehenden und zwei bewegten Hindernissen. Dieses Szenario soll die Leistungsfähigkeit der Verfahren in komplexen und kritischen Verkehrssituationen mit mehreren bewegten Hindernissen zeigen. In Abbildung 5.8 oben beträgt die initiale Geschwindigkeit $60 \mathrm{~km} / \mathrm{h}$. In dieser Situation ist die Kollision zum Zeitpunkt der Auslösung durch ein reines Bremsmanöver vermeidbar. Ohne ein Regelwerk oder eine Logik wird diese Lösung vom OTO gewählt. Wird die initiale Geschwindigkeit erhöht (siehe Abbildung 5.8 Mitte), ist zum Zeitpunkt der Auslösung kein Bremsmanöver mehr kollisionsfrei durchführbar. Stattdessen wird ein kombiniertes Brems- und Lenkmanöver durchgeführt und das Ego-Fahrzeug kommt nach dem Spurwechsel zum Stehen. In Abbildung 5.8 unten ist das Ergebnis bei einer höheren Eingangsgeschwindigkeit zu sehen. Es wird erneut ein Brems-Ausweich-Manöver durchgeführt. Aufgrund der höheren Geschwindigkeit bleibt das Ego-Fahrzeug nicht zwischen dem ersten und zweiten Hindernis stehen. Stattdessen wird der zweite Spurwechsel durchgeführt und anschließend angehalten. Die Bremsung in den Stillstand erfolgt jedoch bevor die beiden nebeneinander stehenden Hindernisse 3 und 4 dies erfordern. Der Grund liegt in der Minimierung des Rucks, was dazu führt, dass eine einmal erreichte Verzögerung nur langsam wieder abgebaut werden kann. Das Verhältnis der entsprechenden Gewichte bestimmt, wie die Beschleunigungen auf- und abgebaut werden. In dieser Situation könnte das Verhalten durch eine entsprechend an- 

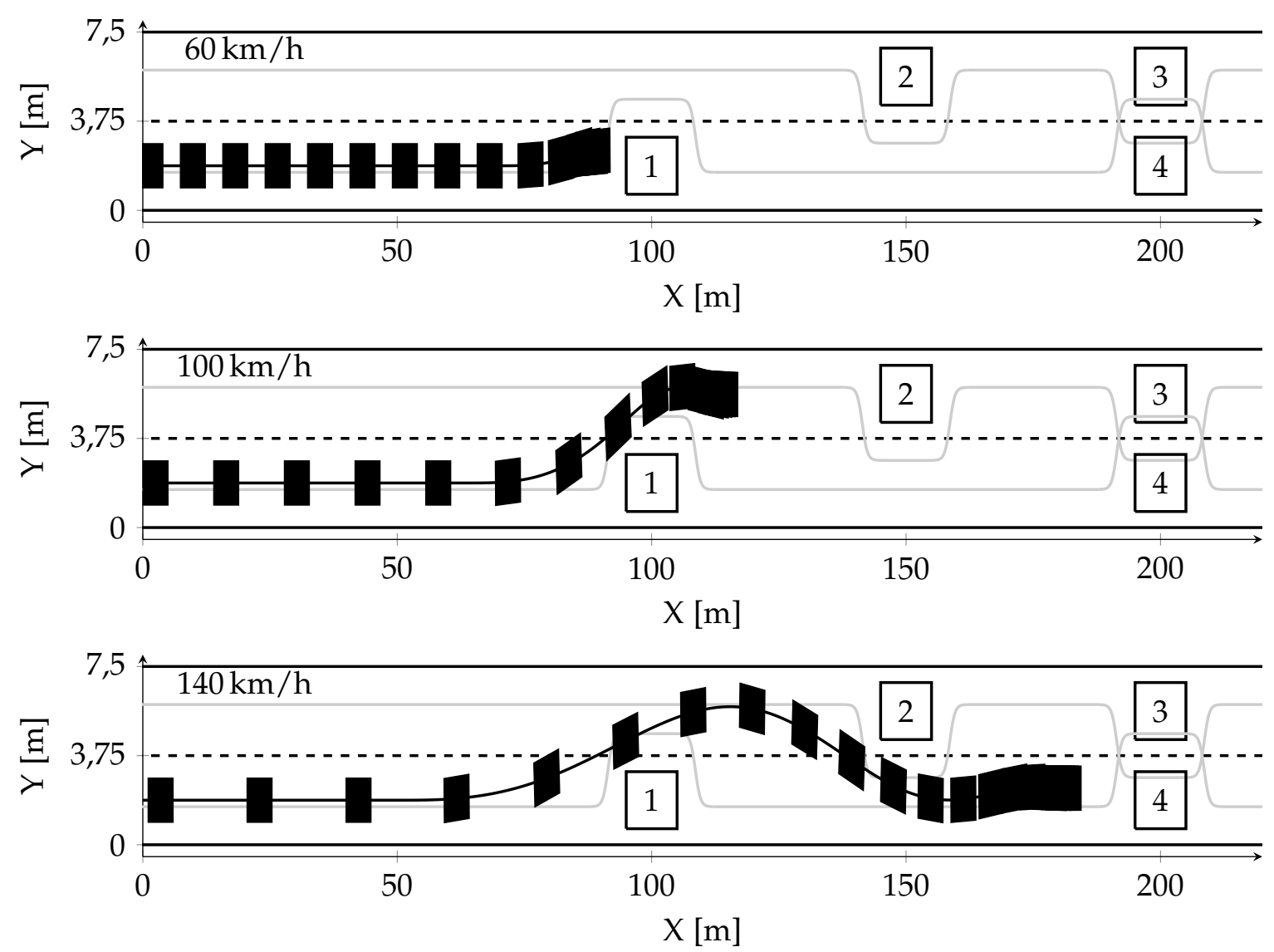

Abbildung 5.8.: Simulationsergebnisse bei unterschiedlichen initialen Geschwindigkeiten $v_{\text {init }}=60,100$ und $140 \mathrm{~km} / \mathrm{h}$. Das Ego-Fahrzeug ist in Abständen von 0,5s durch schwarz ausgefüllte Vierecke, Hindernisse durch schwarz gerahmte Rechtecke gekennzeichnet. Das Umfeldmodell ist durch hellgraue Linien dargestellt.

gepasste Wahl der Gewichte verbessert werden. Jedoch würde das negative Folgen für andere Situation haben, in denen die Solltrajektorie ungenauer realisiert werden würde. Hier zeigt sich, dass das Massenpunktmodell die wirkliche Dynamik der Regelstrecke grundsätzlich nicht exakt sondern nur tendenziell berücksichtigen kann. Dies ist auch der Grund, warum das Umfeldmodell nicht exakt berücksichtigt wird, sondern die Schwerpunktsbahn (schwarze Linie) die Begrenzunglinie des Umfeldmodells (grau Linien) schneidet. Dies ist insbesondere beim Ausweichmanöver vor dem ersten Hindernis zu erkennen. In Abbildung 5.9 ist das Szenario mit den bewegten Hindernissen zu erkennen und Abbildung 5.10 zeigt die zugehörigen dynamischen Größen. Auch dieses Manöver wird kollisionsfrei und stabil durchfahren. Aufgrund der nicht exakten Realisierung ist der minimale Abstand zum entgegenkommenden Fahrzeug jedoch sehr klein. Der Verlauf des Solllenkradwinkels zeigt zu Beginn des Manövers eine hohe Änderungsrate, welche ein maximal schnelles Ansteigen des Lenkradwinkels unmittelbar nach der Auslösung des Manövers bewirkt und auf dem sprungförmigen Verlauf der Regelgrößen am Vorausschaupunkt begründet ist. Anschließend verlaufen sowohl der Soll- und Istlenkradwinkel als auch der Soll- und Istschlupf gleichmäßig und ohne große Abweichungen. Die Phasenebene zeigt die stabile Fahrzeugführung trotz der hohen Kritikalität. 

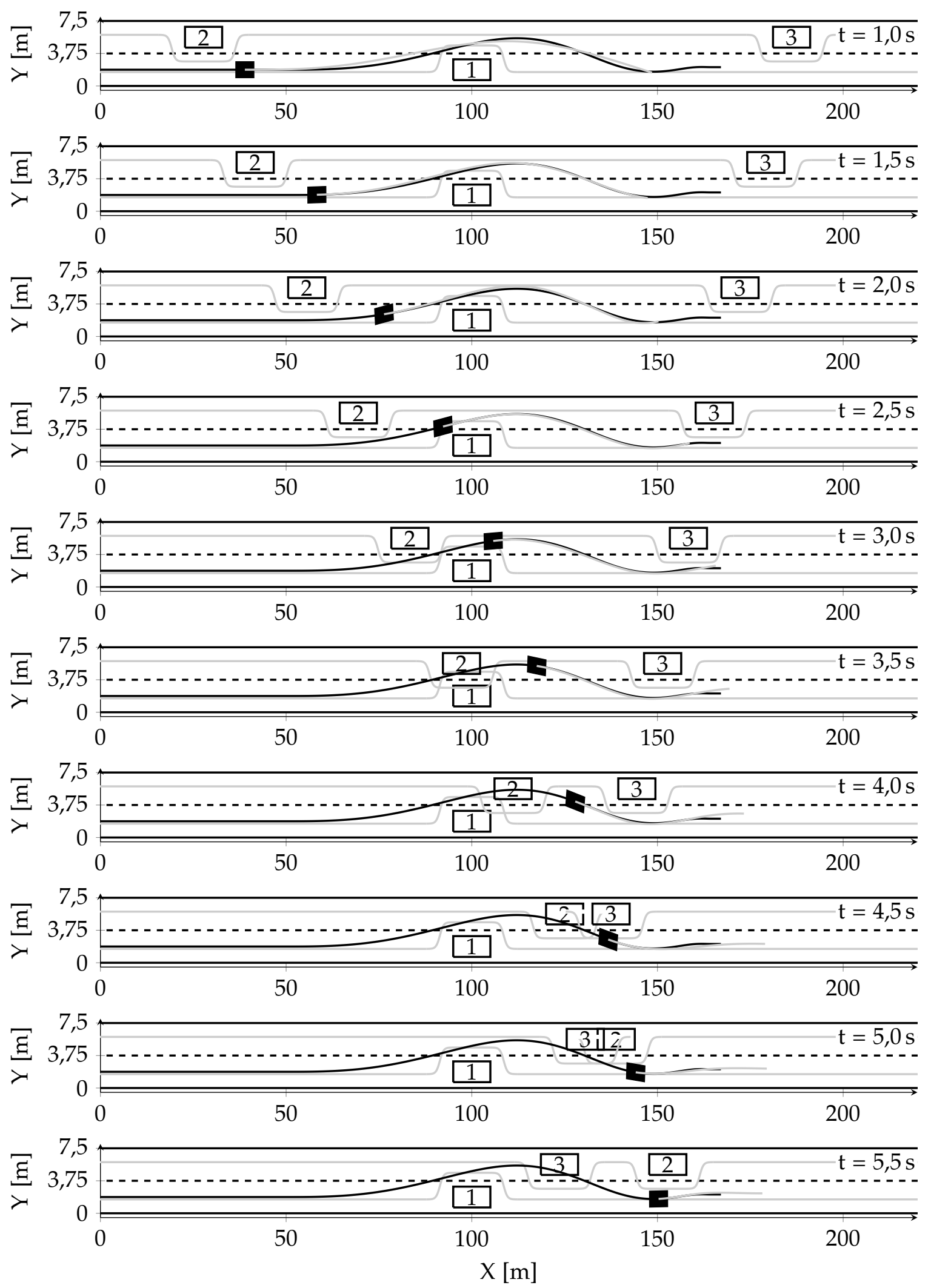

Abbildung 5.9.: Kollisionsvermeidungsmanöver mit einer initialen Geschwindigkeit von $v_{\text {init }}=140 \mathrm{~km} / \mathrm{h}$. Das nach rechts fahrende Hindernis 2 bewegt sich gleichförmig geradlinig mit $100 \mathrm{~km} / \mathrm{h}$, das nach links fahrende Fahrzeug 3 mit $50 \mathrm{~km} / \mathrm{h}$. Zwischen den Abbildungen vergeht eine Zeitspanne von 0,5 s. Die im jeweiligen Zeitschritt geplante Trajektorie und das Umfeldmodell sind in grau, die resultierende Trajektorie ist in schwarz dargestellt. 
Das Minimum des Schlupfes liegt dabei mit etwas Verzögerung hinter dem ersten Nulldurchgang des Lenkradwinkelverlaufs. Das heißt, dass in den Augenblicken, in denen keine hohe Querbeschleunigung benötigt wird, das Beschleunigungspotential in Längsrichtung genutzt wird. Der Aspekt ist auch im Beschleunigungsdiagramm zu sehen.
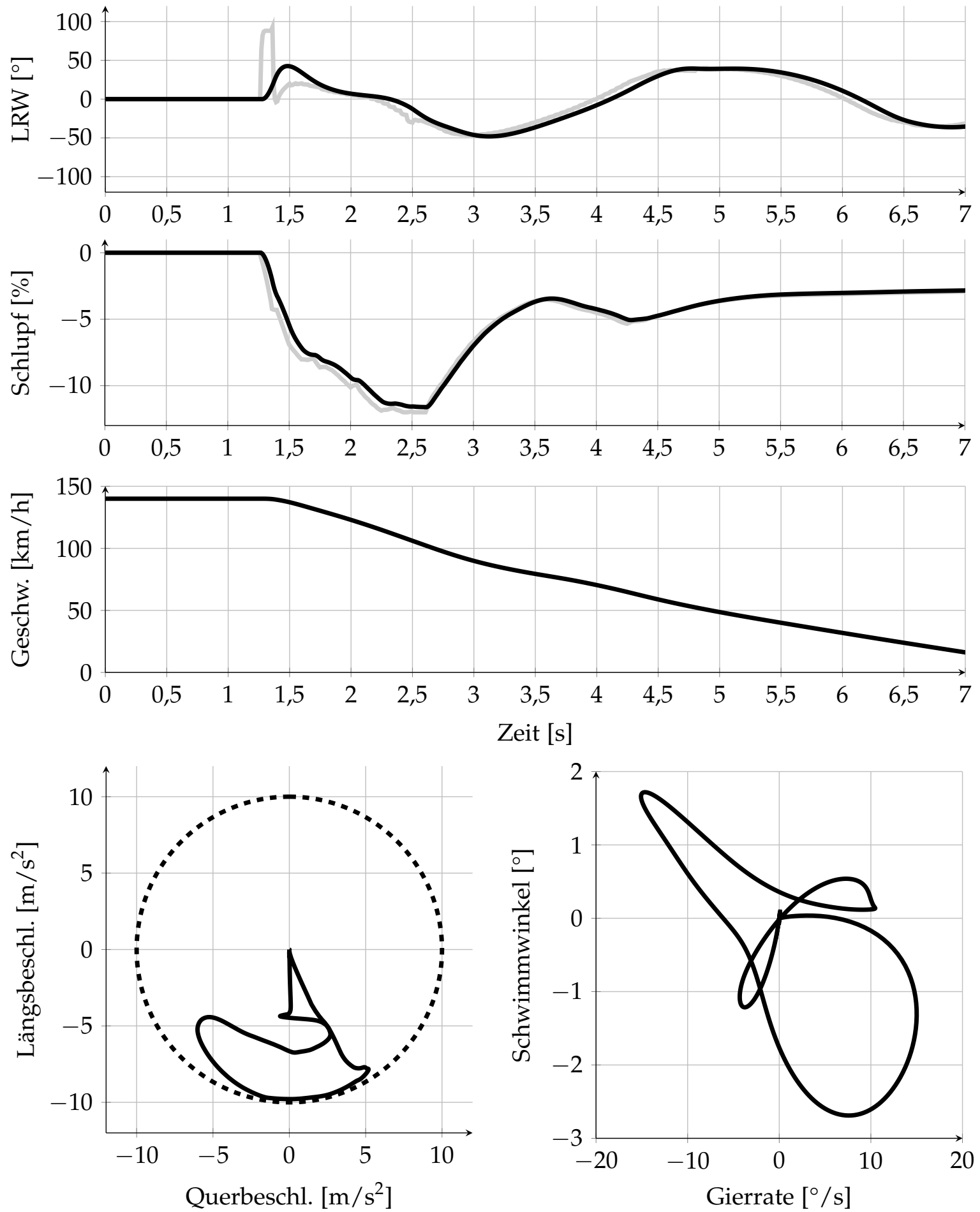

Abbildung 5.10.: Dynamische Größen während eines Kollisionsvermeidungsmanövers mit sich bewegenden Hindernissen bei einer initialen Geschwindigkeit von $v_{\text {init }}=140 \mathrm{~km} / \mathrm{h}$. Sollgrößen sind in grau, Istgrößen in schwarz gezeichnet. 


\title{
6
}

\section{Modellprädiktives Planungs- und Regelungsverfahren}

\begin{abstract}
Das Online-Trajektorienoptimierungsverfahren hat sich als leistungsfähige Vorgehensweise zur Berechnung kollisionsfreier Trajektorien herausgestellt. Das TEB ist jedoch nur optimal bezüglich eines Gütemaßes, welches mehrere kinematische Größen (Ort, Geschwindikeit, Beschleunigung, Ruck) aber nicht die Dynamik des Kraftfahrzeuges berücksichtigt. Besonders bei Notmanövern in kritischen Verkehrssituationen werden die dynamischen Eigenschaften des Fahrzeugs deutlich. Hieraus ergibt sich die Idee, ein Modell der Fahrdynamik (im Sinne eines Prädiktionsmodells) in die Planung und damit in die Optimierung zu integrieren. Später wird gezeigt, dass sich als Lösung des Optimalsteuerungsproblems die optimalen Stellgrößen ergeben und somit die unterlagerten Fahrdynamikregler nicht mehr nötig sind. Zunächst stellt sich jedoch die Frage, wie ein dynamisches Modell in das TEB integriert werden kann. Hierzu werden im Folgenden fünf Möglichkeiten diskutiert.
\end{abstract}

\section{Möglichkeit 1: Erweiterung des TEB}

Zunächst besteht die Möglichkeit, die Punkte $P_{k}$ des TEB um die Orientierung des Fahrzeugs zu erweitern:

$$
P_{k}=\left(\begin{array}{c}
X_{k} \\
Y_{k} \\
\Psi_{k}
\end{array}\right)
$$

Diese Beschreibung erlaubt es, zwischen Gierwinkel, Kurswinkel und Schwimmwinkel zu unterscheiden. Als Folge könnte die Fahrstabilität auf dem Planungshorizont mit dem Ziel untersucht werden, eine stabile Fahrzeugführung zu garantieren. Hierzu ist es naheliegend, den Schwimmwinkel im Gütemaß zu berücksichtigen und zu minimieren. Als Folge ergäbe sich $\beta \equiv 0$. Die zusätzliche Berücksichtigung der Gierrate würde dieses Problem zwar lösen, die Aufteilung der Kursrate in Gierrate und Schwimmwinkeländerung ergäbe sich aber aus dem Verhältnis der Gewichte und nicht etwa aus der Realisierbarkeit. Auf diese Weise wäre das dynamische Verhalten daher nicht berücksichtigt. 


\section{Möglichkeit 2: Modellinversion}

Das Prädiktionsmodell stellt ein Mehrgrößensystem der Form

$$
\dot{\mathbf{x}}=\mathbf{f}(\mathbf{x}, \mathbf{u}), \mathbf{x}(t=0)=\mathbf{x}_{0}
$$

mit dem Zustandsvektor $\mathbf{x}^{\mathrm{T}}=\left(\begin{array}{lllllllll}\beta & \dot{\Psi} & \Psi & X & Y & v & \dot{\delta} & \delta & s_{1}\end{array}\right)$ und dem Ausgangsvektor

$$
\mathbf{y}=\left(\begin{array}{l}
X \\
Y
\end{array}\right)
$$

dar. Dabei entspricht der Ausgangsvektor den vom OTO geplanten Größen. Entsprechend könnte durch Modellinversion der zur Ausweichtrajektorie zugehörige Zustandsund Stellvektor rekonstruiert werden. Im Allgemeinen ist das Modell jedoch nicht invertierbar.

\section{Möglichkeit 3: Erweiterung des TEB und Modellinversion lokal linearer Modelle}

In diesem Fall wird das TEB auf den gesamten Zustandsvektor erweitert. Neben dem Zustandsvektor selbst lassen sich dessen Änderungen entlang des Bandes mit finiten Differenzen berechnen. Um die Stellgrößen zu berechnen, muss die Umkehrfunktion $\mathbf{f}^{-\mathbf{1}}(\dot{\mathbf{x}})$ gebildet werden. Dies ist im Allgemeinen nicht möglich. Wird das Modell jedoch lokal linearisiert, ergeben sich die Stellgrößen aus

$$
\mathbf{u}=\left(\mathbf{B}^{T} \mathbf{B}\right)^{-1} \mathbf{B}^{T}(\dot{\mathbf{x}}-\mathbf{A} \mathbf{x})
$$

unter der Vorraussetzung das $\mathbf{B}^{T} \mathbf{B}$ invertierbar ist. Zudem muss die Planung der Zustandstrajektorien und deren Änderung realisierbar sein. Dies wird jedoch bisher nicht im Gütemaß berücksichtigt. Vielmehr wird Gleichung (6.0.4) nicht auswertbar sein, falls nichtrealisierbare Zustandstrajektorien geplant werden. Gütemaßanteile, welche die Realisierbarkeit sicher stellen, sind bei dieser Formulierung aufwändig und nicht recheneffizient $\mathrm{zu}$ bestimmen.

\section{Möglichkeit 4: Modellprädiktive Regelung - Sequentielle Formulierung}

Anstatt wie bei den vorherigen Möglichkeiten die zur Trajektorie zugehörigen Zustands- und Stellgrößen zu rekonstruieren, wird bei der modellprädiktiven Regelung die logische Wirkrichtung umgekehrt. Bei der Formulierung als Optimalsteuerungsproblem werden die Stellgrößen als Optimierungsvariablen gewählt und die Zustandstrajektorien ergeben sich durch die Lösung der Zustandsdifferentialgleichungen mit einem geeigneten numerischen Integrationsverfahren. Das Gütemaß enthält Anteile zur Kollisionsvermeidung und Minimierung der Fahrdynamik. Als Lösung des Optimalsteuerungsproblems ergeben sich neben kollisionsfreien Trajektorien auch die zugehörigen Stellgrößen. Damit werden die unterlagerten Fahrdynamikregler überflüssig. Das Verfahren löst also die bisher getrennt behandelten Aufgaben Trajektorienplanung und -folgeregelung in einem Schritt. Daher wird in dieser Arbeit von modellprädiktiver Planung und Regelung gesprochen, auch wenn die Schreibweise des Problems mathematisch der eines MPC (also einem Regelungsverfahren) entspricht. Die Architektur des Verfahrens ist in Abbildung 6.1 dargestellt. Das Verfahren weist die üblichen Vorteile der modellprädiktiven Regelung auf: 


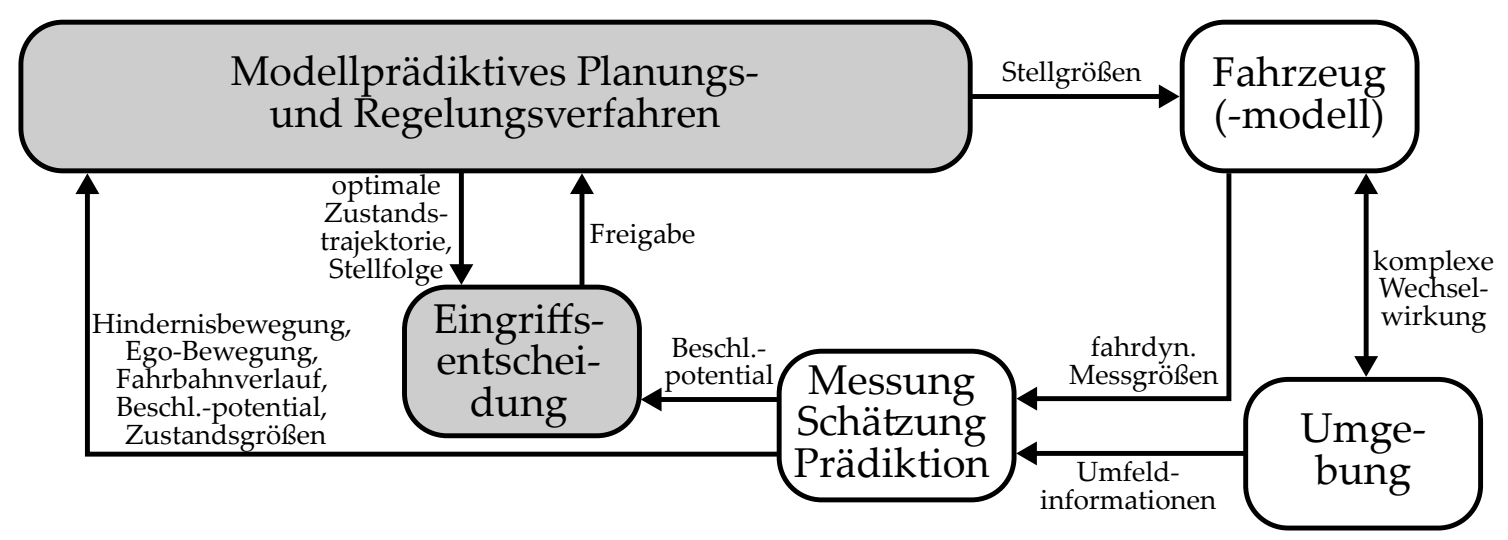

Abbildung 6.1.: Architektur des modellprädiktiven Planungs- und Regelungsverfahrens.

- Es handelt sich um einen Optimalregler.

- Die Streckendynamik wird explizit zur Laufzeit berücksichtigt und nicht nur implizit während der Designphase des Reglers.

- Stell- und Zustandsbegrenzungen werden explizit berücksichtigt.

- Die erreichbare Regelungsgüte ist sehr hoch.

- Das Verfahren ist einfach zu einem adaptiven Regelungssystem erweiterbar, falls die sich ändernden Parameter des Prädiktionsmodells bekannt sind bzw. online identifiziert werden.

- Bekannte Störgrößen können bei der Planung explizit berücksichtigt werden.

Die wesentliche Herausforderung ist die Lösung des Optimalsteuerungsproblems in Echtzeit. Anders als beim TEB liegt kein dünnbesetztes Optimierungsproblem vor. Die fünfte Möglichkeit zeigt auf, wie das Optimalsteuerungsproblem angepasst werden kann, um eine Formulierung mit dünnbesetzter Jakobi- bzw. Hessematrix zu erreichen.

\section{Möglicheit 5: Modellprädiktive Regelung - simultane Formulierung}

Bei der simultanen Formulierung werden neben den Stellgrößen auch die Zustandsgrößen als Optimierungsvariablen gewählt. Da diese Größen jedoch nicht unabhängig voneinander sind, wird die Einhaltung der Zustandsdifferentialgleichung als Nebenbedingung formuliert. Es ergibt sich ein Optimalsteuerungsproblem höherer Ordnung als bei der sequentiellen Formulierung. Dieses dünnbesetzte Optimierungsproblem weist einen geringeren Rechenaufwand im Vergleich zur sequentiellen Formulierung auf, da sich eine schnellere Konvergenz ergibt (siehe [Rös+15b] und [Rös+14] $)$. In der Arbeit wird daher die simultane Formulierung verwendet. 


\subsection{Formulierung des Optimalsteuerungsproblems}

Die optimalen Zustandstrajektorien $\tilde{\mathbf{x}}^{*}$ und Stellgrößenverläufe $\tilde{\mathbf{u}}^{*}$ ergeben sich aus der Lösung des Optimalsteuerungsproblems

$$
(\tilde{\mathbf{x}}, \tilde{\mathbf{u}})^{*}=\arg \min _{\tilde{\mathbf{x}}, \tilde{\mathbf{u}}} J_{\mathrm{MPPC}}
$$

mit den Nebenbedingungen

$$
\begin{aligned}
\mathbf{x}_{0}-\tilde{\mathbf{x}}_{0} & =\mathbf{0} & & \text { (fester Anfangswert) } \\
\tilde{\mathbf{x}}_{k+1}-\tilde{\mathbf{x}}_{k}-\mathbf{f}\left(\tilde{\mathbf{x}}_{k}, \tilde{\mathbf{u}}_{k}\right) \Delta t & =\mathbf{0} & & \text { (Gleichheitsnebenbedingung) und } \\
\mathbf{g}\left(\tilde{\mathbf{x}}_{k}, \tilde{\mathbf{u}}_{k}\right) & \geq \mathbf{0} & & \text { (Ungleichheitsnebenbedingungen). }
\end{aligned}
$$

Die Prädiktion startet vom aktuell gemessenen bzw. beobachteten Zustandsvektor. Die Gleichheitsnebenbedingung bewirkt, dass die zunächst unabhängig wählbaren Zustände $\tilde{\mathbf{x}}_{k}$ und Stellgrößen $\tilde{\mathbf{u}}_{k}$ die Zustandsdifferentialgleichungen des Prädiktionsmodells erfüllen. Dies wird durch das in Abbildung 6.2 graphisch dargestellte Mehrfachschießverfahren näherungsweise erreicht. Hierbei wird jeder geplante Zustandsvektor als

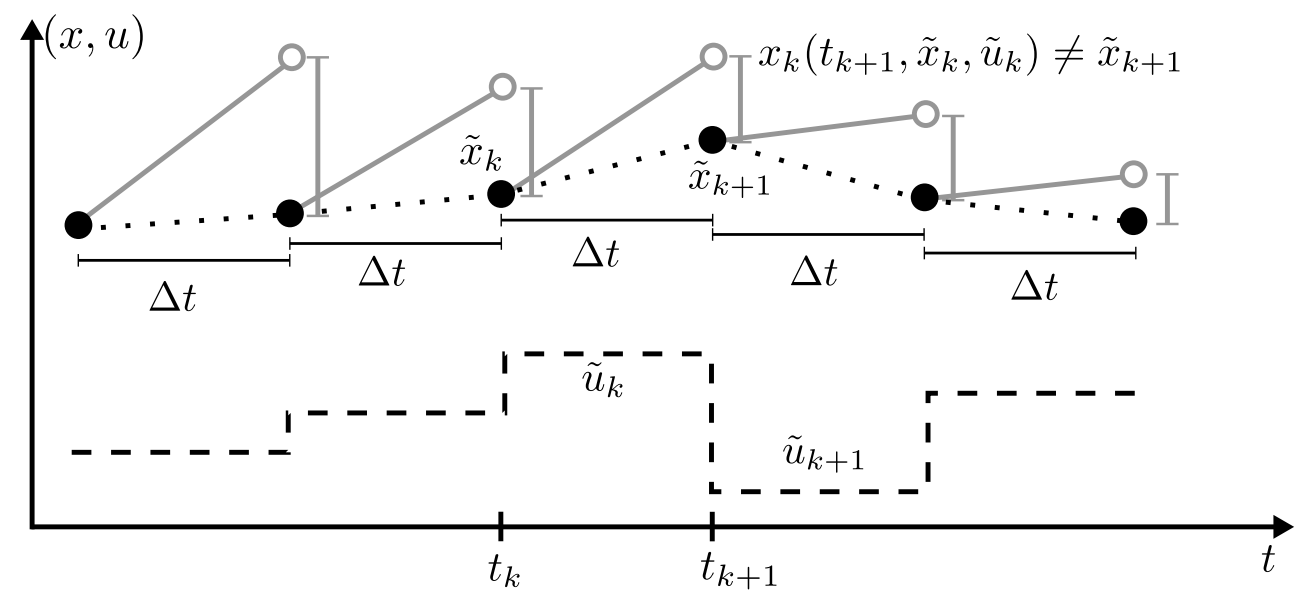

Abbildung 6.2.: Darstellung des Mehrfachschießverfahrens.

Startwert zur Lösung eines Anfangswertproblems (AWP) aufgefasst. Das AWP wird im vorliegenden Fall zuverlässig durch das Euler-Vorwärts-Verfahren gelöst. Der Integrationsschritt wird unter Berücksichtigung der Stellgrößen durchgeführt. Der so erhaltene Endwert muss dem nächsten geplanten Zustandsvektor entsprechen. Die Ungleichheitsnebenbedingungen in Gleichung (6.1.2) beinhalten weitere Beschränkungen bzw. Bestrafungen der Stell- oder Zustandsgrößen bzw. deren Änderung. Zum Beispiel werden die Zustandsgrößen $(X, Y)$ im Sinne der Kollisionsvermeidung beschränkt. Um das Optimalsteuerungsproblem zu lösen, werden die Nebenbedingungen in das Gütemaß integriert und mit hohen Gewichtungen versehen. Dadurch werden die Nebenbedingungen nicht exakt erfüllt (weiche Nebenbedingungen - Soft-Constraints). Der Vorteil dieser Vorgehensweise ist, dass das Optimierungsproblem nicht beschränkt ist, was die Lösung vereinfacht und sich durch die weiche Gleichheitsnebenbedingung wie beim OTO wieder eine dünn besetzte Jakobimatrix ergibt. Dies wird von dem 
verwendeten MPC Framework (siehe $[\overline{\text { Rös+15b] }}$ und [ $[\overline{R o ̈ s+14}]$ ) effizient ausgenutzt. Ein weiterer Vorteil ist, dass die initiale Lösung nicht die Nebenbedingungen erfüllen muss. Die Algorithmen der Umfelderfassung erkennen Hindernisse teilweise erst innerhalb des Prädiktionshorizontes. Aus der Sicht des Planungsalgorithmus erscheinen Hindernisse dadurch plötzlich, was dazu führen kann, dass die initiale Lösung des Optimalsteuerungsproblems, welche sich aus der optimalen Lösung im vorangegangenen Abtastschritt ergibt, durch ein Hindernis verläuft und somit die Nebenbedingungen verletzt. Die Fähigkeit, Verletzungen von Nebenbedingungen behandeln zu können, ist daher von grundlegender Bedeutung.

\subsection{Entwurf des Gütemaßes zur Kollisionsvermeidung}

\subsubsection{Aufbau des Gütemaßes}

Das oben beschriebene restringierte Optimierungsproblem wird wie in Kapitel 5 durch die Verwendung von Soft-Constraints zu einem unbeschränkten Problem. Das Gütemaß ergibt sich unter dieser Voraussetzung zu:

$$
\begin{aligned}
J_{\mathrm{MPPC}} & =\sum_{k=1}^{n_{\mathrm{p}}-1} \gamma_{\text {sys }}^{i t} h\left(\tilde{\mathbf{x}}_{k}, \tilde{\mathbf{x}}_{k+1}, \tilde{\mathbf{u}}_{k}\right)+\sum_{k=1}^{n_{\mathrm{p}}} \gamma_{\mathrm{env}}^{i t} \boldsymbol{\Gamma}_{\mathrm{env}}\left(\tilde{\mathbf{x}}_{k}\right)+\sum_{k=1}^{n_{\mathrm{p}}-1} \gamma_{\mathrm{u}}^{i t} g_{\mathrm{u}}\left(\tilde{\mathbf{u}}_{k}\right)+\cdots \\
& +\tilde{\mathbf{x}}_{\mathrm{n}_{\mathrm{p}}}^{\mathrm{T}} \mathbf{Q}_{\mathrm{n}_{\mathrm{p}}} \tilde{\mathbf{x}}_{\mathrm{n}_{\mathrm{p}}}+\sum_{k=1}^{n_{\mathrm{p}}-1}\left(\tilde{\mathbf{x}}_{k}^{\mathrm{T}} \mathbf{Q} \tilde{\mathbf{x}}_{k}+\tilde{\mathbf{u}}_{k}^{\mathrm{T}} \mathbf{R} \tilde{\mathbf{u}}_{k}\right)+\sum_{k=1}^{n_{\mathrm{p}}-1} \dot{\tilde{\mathbf{x}}}_{k}^{\mathrm{T}} \mathbf{S} \dot{\tilde{\mathbf{x}}}_{k} .
\end{aligned}
$$

Durch den Exponenten it wird das Gewicht nach einer Anzahl an Iterationen schrittweise während der Optimierung erhöht. Die Einhaltung der Systemdynamik wird durch die Gleichung

$$
h\left(\tilde{\mathbf{x}}_{k}, \tilde{\mathbf{x}}_{k+1}, \tilde{\mathbf{u}}_{k}\right)=\left(\tilde{\mathbf{x}}_{k+1}-\tilde{\mathbf{x}}_{k}-\mathbf{f}\left(\tilde{\mathbf{x}}_{k}, \tilde{\mathbf{u}}_{k}\right) \Delta t\right)^{T} \mathbf{P}\left(\tilde{\mathbf{x}}_{k+1}-\tilde{\mathbf{x}}_{k}-\mathbf{f}\left(\tilde{\mathbf{x}}_{k}, \tilde{\mathbf{u}}_{k}\right) \Delta t\right)
$$

beschrieben. Das Umfeldmodell $\Gamma_{\text {env }}$ ist identisch mit dem in Kapitel 5. Der dritte Anteil enthält die Stellgrößenbeschränkung.

$$
g_{u}\left(\tilde{\mathbf{u}}_{k}\right)= \begin{cases}s_{\mathrm{soll}, k}^{2} & \text { wenn } s_{\mathrm{soll}, k}>0 \\ 0 & \text { wenn } s_{1, \mathrm{~min}} \leq s_{\mathrm{soll}, k} \leq 0 \\ \left(s_{\mathrm{soll}, k}-s_{1, \mathrm{~min}}\right)^{2} & \text { wenn } s_{\mathrm{soll}, k}<s_{1, \mathrm{~min}}\end{cases}
$$

Hierdurch wird der Sollschlupf zum einen auf negative Werte (Verzögerungen) und zum anderen auf Werte oberhalb des Minimums der Reifenkennlinie $s_{1, \text { min }}$ beschränkt. Die Beschränkung des Solllenkradwinkels hat sich als nicht notwendig herausgestellt, da die Untersteuergrenze nicht verletzt wird. Desweiteren enthält das Gütemaß den üblichen Bolzaschen Anteil, welcher aus einem Mayer-Term zur Bewertung des Endzustandes und einem Lagrangeschen Anteil für verlaufs- bzw. verbrauchsoptimale Lösungen besteht. Abschließend wird die Änderung der Zustandsgrößen bestraft, wobei Vorwärtsdifferenzen zur Approximation der Ableitung verwendet werden:

$$
\dot{\tilde{\mathbf{x}}}_{k}=\frac{\tilde{\mathbf{x}}_{k+1}-\tilde{\mathbf{x}}_{k}}{\Delta t}
$$




\subsubsection{Zielkonflikte zwischen Gütemaßanteilen}

Die Anteile des Gütemaßes ergeben sich aus verschiedenen Anforderungen und Zielen, die sich teilweise entgegenstehen können. Es muss ein Kompromiss zwischen den Zielen gefunden werden, welcher durch das Verhältnis der beteiligten Gewichtungsfaktoren definiert ist. Das Gütemaß nach Gleichung (6.2.1) weist die folgenden Anteile auf:

- Einhaltung der Systemdynamik,

- Umfeldmodell,

- Einhaltung der Stellgrößenbeschränkung,

- Lagrange-Term,

- Mayer-Term und

- Restterme.

Die ersten drei Anteile ergeben sich aus den Nebenbedingungen und stellen Beschränkungen mit hohen Gewichtungen dar. Die letzten drei Anteile sind Bestrafungen mit wesentlich geringeren Gewichtungen, welche das Verhalten innerhalb der Begrenzungen definieren. Diese Anteile werden in dieser Arbeit erweiterter Bolza-Term genannt. Abbildung 6.3 zeigt die Gruppierung und Wechselwirkung der Gütemaßanteile. Zwischen den einzelnen Soft-Constraints treten keine Zielkonflikte auf, solange

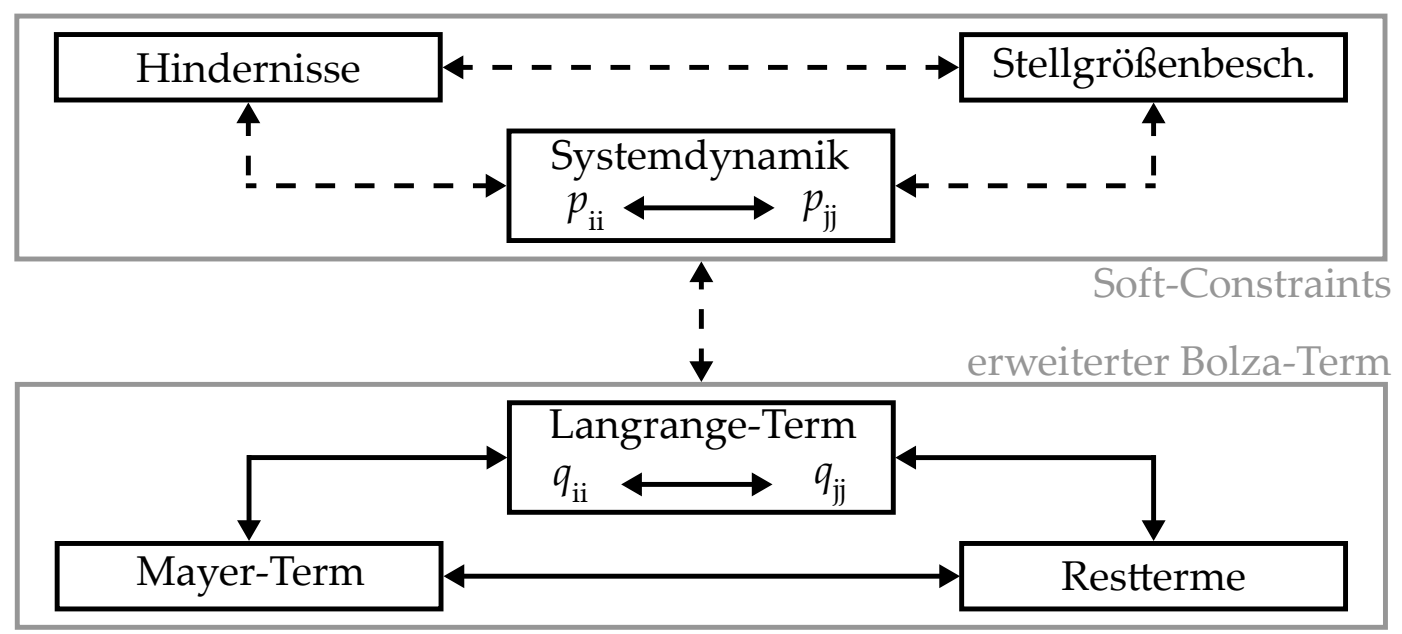

Abbildung 6.3.: Visualisierung der Gütemaßanteile und der auftretenden Zielkonflikte. Durchgezogene Pfeile symbolisieren uneindeutige, gestrichelte Pfeile eindeutige Zielkonflikte.

ein kollisionsfreies Manöver innerhalb der Stellgrößenbeschränkung möglich ist, da die drei Nebenbedingungen gleichzeitig erfüllt werden können. Es treten jedoch zum Teil Zielkonflikte zwischen den internen Gewichtungen der Systemdynamik $p_{i j}$ auf. Die Vorgehensweise zur Wahl dieser Gewichte wird im nächsten Abschnitt beschrieben. Zwischen den weichen Nebenbedingungen und dem erweiterten Bolza-Term können sich, je nach Verkehrssituation, Zielkonflikte ergeben. Diese sind jedoch durch das 
Konzept der weichen Nebenbedingung gelöst, sodass die Beiträge des erweiterten Bolza-Terms rezessiv sind. Innerhalb des erweiterten Bolza-Terms ergeben sich mehrere Zielkonflikte. Einige dieser Zielkonflikte unterliegen der subjektiven Bewertung und können daher auch manuell gewählt werden. Als Beispiel kann die Ausregelung eines Anfangszustandes schnell und mit Überschwingen oder langsamer und gedämpft erfolgen. Welche Lösung die Passagiere bevorzugt wählen würden, unterliegt ihrer subjektiven Beurteilung.

\subsubsection{Wahl der Gewichtungsfaktoren}

Wie der vorherige Abschnitt erläutert hat, ist der Kompromiss zwischen weichen Nebenbedingungen und Bestrafungen so zu wählen, dass die weichen Nebenbedingungen dominant sind. Die entsprechenden Gewichtungen $\gamma_{\text {sys }}^{i t} \gamma_{\text {env }}^{i t}$ und $\gamma_{u}^{i t}$ können daher heuristisch gewählt werden. Auf nicht heuristischem Wege müssen die Gewichte $\mathbf{P}$ gewählt werden, da die Gewichtsverteilung zur gleichmäßigen Einhaltung der Systemdynamik unbekannt ist. Die Gewichtungen $\mathbf{Q}, \mathbf{Q}_{\mathrm{f}}, \mathbf{R}$ und $\mathbf{S}$ sind hingegen Designparameter, welche manuell gewählt werden, um ein bestimmtes Verhalten zu erzielen. Die Wahl von $\mathbf{P}$ hängt nur vernachlässigbar vom erweiterten Bolza-Term $\mathrm{ab}$, da $\mathbf{P}$ Bestandteil eines Soft-Constraints ist. Die Elemente von P sollten möglichst keinen Einfluss auf das Verhältnis von Quer- und Längsführungsanteilen haben. Es muss daher eine Gewichtung gefunden werden, welche die Einhaltung der geplanten Zustandsverläufe mit gleicher Genauigkeit bewirkt. Die Gewichte werden initial so gewählt, dass die zugehörigen Größen auf ihre typischen Maximalwerte normiert und anschließend mit einem genetischen Algorithmus optimiert werden. Das Optimierungsproblem lautet:

$$
\mathbf{P}^{*}=\arg \min _{\mathbf{P}} \sqrt[E_{\mathrm{g}}]{\frac{\sum_{i=1}^{n} \operatorname{NRMSE}(\tilde{\mathbf{x}}, \mathbf{x})_{i}^{E_{\mathrm{g}}}}{n}}+S T
$$

Die Funktion ST bestraft kollisionsbehaftete Manöver. Zusätzlich wird durch Normierung sichergestellt, dass

$$
\sum_{i=1}^{n} p_{i i}=1
$$

gilt. So wird die Einhaltung der Systemdynamik aufgeteilt in die absolute Gewichtung durch $\gamma_{\text {sys }}^{i t}$ und die relative Verteilung dieses Gewichtes auf die einzelnen ZustandsgröBen durch P. Es wird in einer Verkehrssituation für einen Abtastschritt das Optimalsteuerungsproblem gelöst und anschließend die Steuerfolge auf das Prädiktionsmodell angewendet. Die Optimierung im offenen Regelreis reduziert den Rechenaufwand gegenüber dem geschlossenen Regelkreis. Zwischen den geplanten und realisierten Zustandstrajektorien ergibt sich eine relative Abweichung, welche sich im NRMSE ausdrückt. Durch diese Wahl ist der Einfluss der gewählten Verkehrssituation reduziert. Es besteht die Anforderung, dass alle Zustandsgrößen ausreichend angeregt werden, da sonst die Berechnung des NRMSE nicht möglich ist (Division durch Null). Die Verkehrssituation darf daher weder durch ein reines Lenk- noch durch ein reines Bremsmanöver gelöst werden können. Die gewählte Verkehrssituation ist in Abbildung 


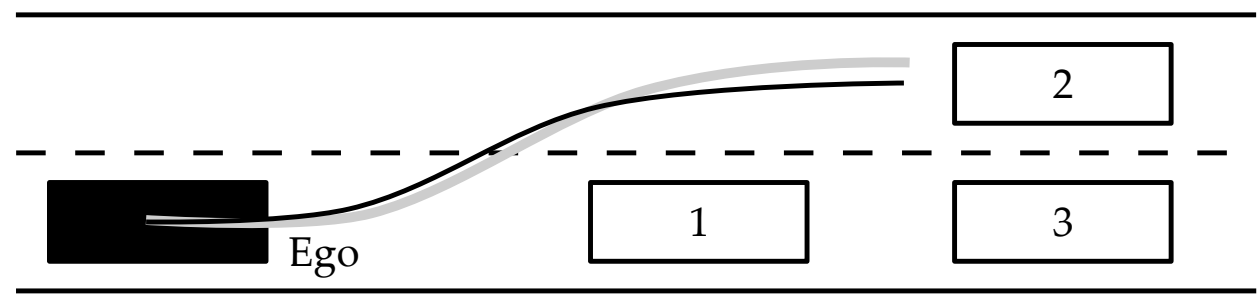

Abbildung 6.4.: Verkehrssituation für das Optimieren der Gewichtungen. Die drei Hindernisse sind als schwarze Rechtecke dargestellt, das Ego-Fahrzeug als ausgefülltes Rechteck, die geplante Trajektorie ist hellgrau und die im offenen Regelkreis realisierte Trajektorie ist als schwarze Linie dargestellt.

6.4 dargestellt. Die Anfangsgeschwindigkeit wird so gewählt, dass es nicht möglich ist vor dem ersten Hindernis anzuhalten. Die Hindernisse 2 und 3 verhindern ein Manöver ohne Bremseingriff. Der Prädiktionshorizont muss dabei ausreichend groß gewählt werden, sodass die Hindernisse 2 und 3 einen Einfluss auf die Planung haben.

\subsubsection{Kritikalitätsmaß und Eingriffsentscheidung}

Da beim MPPC sowohl die vollständige Zustandstrajektorie als auch die Stellfolgen geplant werden, stehen entsprechend viele Informationen zur Verfügung, um die Kritikalität einer Verkehrssituation basierend auf dem Nutzungsgrad der Bewegungspotentiale $\mathrm{zu}$ approximieren (siehe Definition A.1.14). Aufgrund der Allgemeingültigkeit und der besseren Vergleichbarkeit wird analog zum OTO in Kapitel 5 die maximale Gesamtbeschleunigung der Trajektorie nach Gleichung (3.1.5) als Kritikalitätsindikator nach Gleichung (5.2.10) verwendet.

\subsection{Ergebnisse}

In der Abbildung 6.5 wird eine Verkehrssituation mit vier stehenden Hindernissen und den initialen Geschwindigkeiten 60, 100 und $140 \mathrm{~km} / \mathrm{h}$ untersucht. In Abbildung 6.5 oben bewegt sich das Ego-Fahrzeug mit $60 \mathrm{~km} / \mathrm{h}$ auf die Hindernisse zu. Überschreitet die Gesamtbeschleunigung der geplanten Zustandstrajektorie einen Grenzwert, wird das Manöver ausgelöst und die Stellgrößen werden auf die Regelstrecke angewendet. $\mathrm{Zu}$ diesem Zeitpunkt ist bei dieser Geschwindigkeit ein reines Bremsmanöver ohne Kollision möglich. Die Gewichte des erweiterten Bolza-Terms sind so gewählt, dass in einer derartigen Situation ein Bremsmanöver durchgeführt wird. Das EgoFahrzeug hält vor dem Hindernis an. Da ein Bremsmanöver im Vergleich zu einem Ausweichmanöver deutlich einfacher durchzuführen ist und auch weniger von der Unsicherheit der Umfelderfassung abhängt, ist das Bremsmanöver dem Ausweichmanöver vorzuziehen, solange es kollisionsfrei durchführbar ist. Die Querführung beschränkt sich auf kleine Lenkradwinkel und entsprechende Querbeschleunigungen. Durch die leicht gekrümmte Bahn wird der Weg, welcher zum Anhalten zur Verfügung steht, verlängert. Die aufgrund des Querschlupfes reduzierten maximalen Reifenlängskräfte werden durch das Prädiktionsmodell berücksichtigt und sind bei diesen kleinen Querbeschleunigungen vernachlässigbar, sodass sich der Bremsweg 

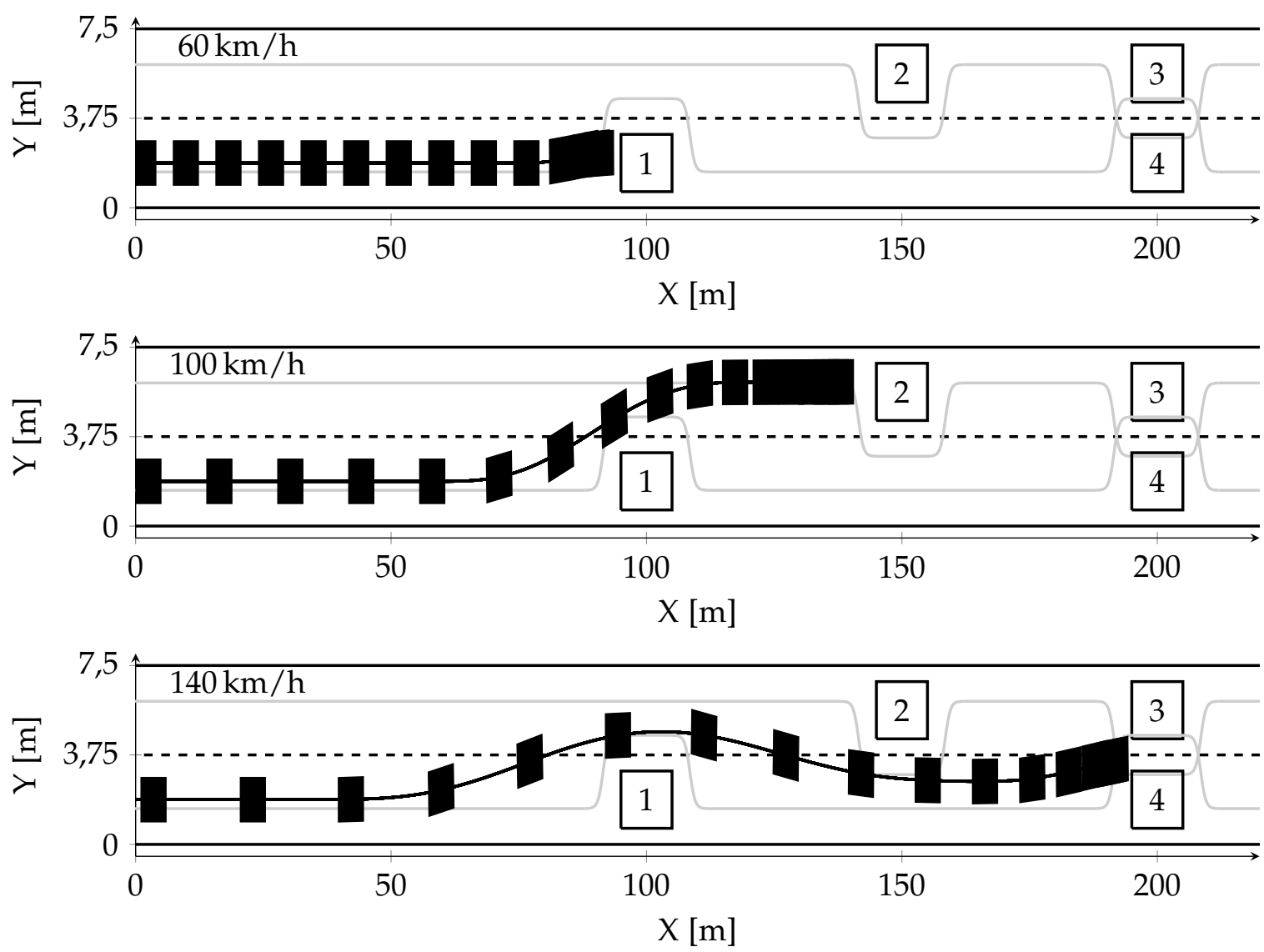

Abbildung 6.5.: Ergebnisse in einer Verkehrssituation mit vier statischen Hindernissen bei unterschiedlichen initialen Geschwindigkeiten: $v_{\text {init }}=60,100$ und 140 km/h. Das Ego-Fahrzeug ist in Abständen von 0,5s abgebildet.

nicht merklich verlängert. In Abbildung 6.5 mittig wird die gleiche Verkehrssituation mit einer initialen Geschwindigkeit von $100 \mathrm{~km} / \mathrm{h}$ betrachtet. Zum Auslösezeitpunkt ist die Kollision durch ein reines Bremsmanöver nicht zu vermeiden. Als Folge wird ein Bremsausweichmanöver mit kombinierter Längs- und Querführung durchgeführt. Anschließend erzwingt das zweite Hindernis die Bremsung in den Stillstand, was aufgrund der Reduktion der Fahrzeuggeschwindigkeit durch das Abbremsen während des Ausweichvorgangs vor dem ersten Hindernis möglich ist. In Abbildung 6.5 unten beträgt die Geschwindigkeit $v_{\text {init }}=140 \mathrm{~km} / \mathrm{h}$. Wie bei dem Ergebnis aus Abbildung 6.5 mittig ist ein reines Bremsmanöver zum Auslösezeitpunkt nicht möglich. Aufgrund der höheren Anfangsgeschwindigkeit bleibt das Ego-Fahrzeug jedoch nicht vor dem 2. Hindernis stehen, sondern muss diesem ebenfalls ausweichen. Anschließend zwingen das 3. und 4. Hindernis das Ego-Fahrzeug zum Anhalten. Im Vergleich zu den Ergebnissen aus Kapitel 5 fällt auf, dass die Positionierung des Ego-Fahrzeugs genauer gelingt. Die Schwerpunktbahn schneidet das Umfeldmodell nicht, sondern tangiert es an mehreren Stellen, wodurch die Kollisionsfreiheit sichergestellt ist. Darüber hinaus sind die Anhaltepositionen sehr nah an den Hindernissen, sodass nicht unnötig stark gebremst wird. In Abbildung 6.6 ist die Verkehrssituation dargestellt, in welcher das Ego-Fahrzeug zunächst vor dem stehenden Fahrzeug ausweicht, bevor es aus der sich schließenden Lücke zwischen den bewegten Hindernissen fährt. 

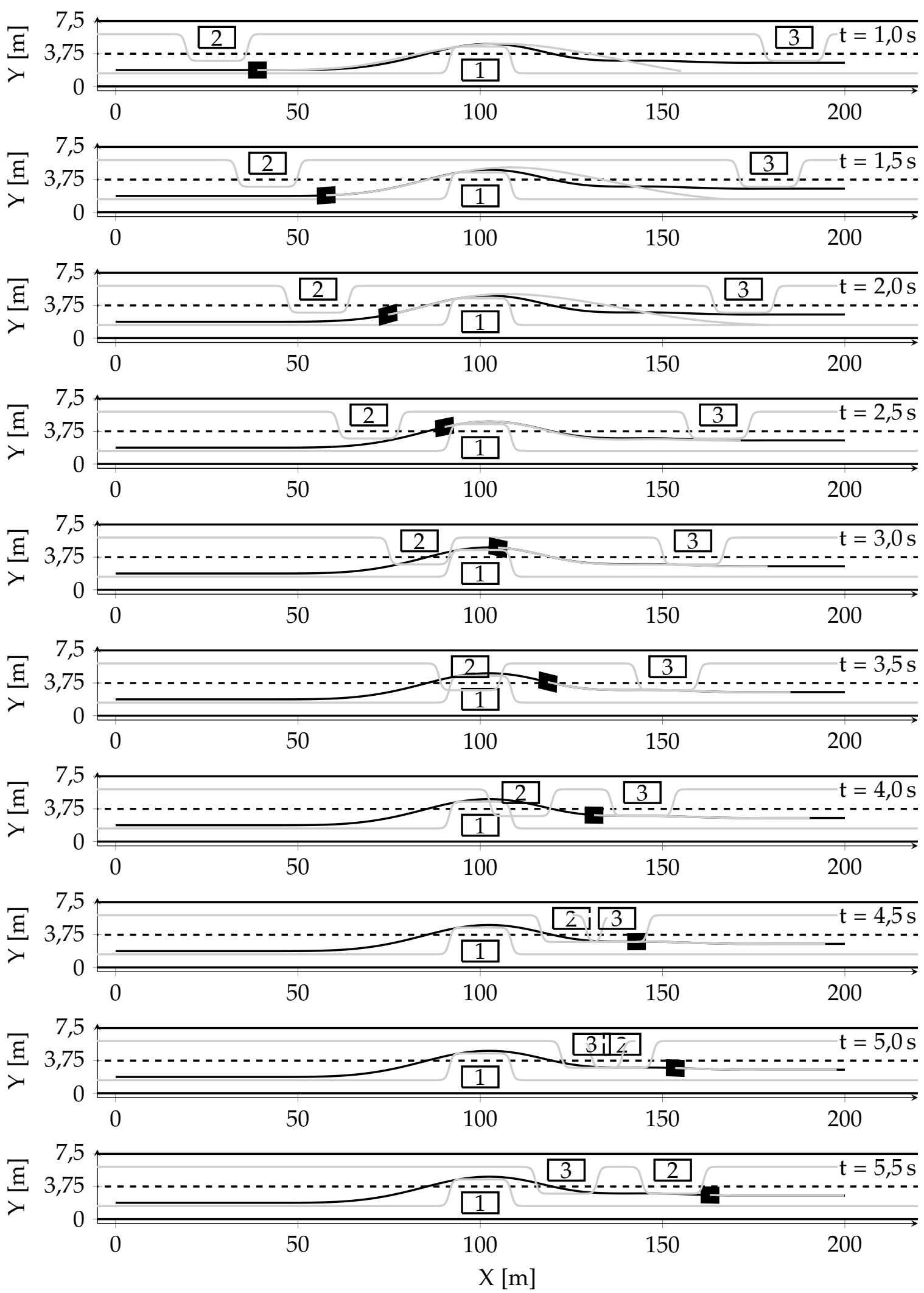

Abbildung 6.6.: Kollisionsvermeidungsmanöver mit einer initialen Geschwindigkeit von $v_{\text {init }}=140 \mathrm{~km} / \mathrm{h}$. Das nach rechts fahrende Hindernis 2 bewegt sich gleichförmig geradlinig mit $100 \mathrm{~km} / \mathrm{h}$, das nach links fahrende Fahrzeug 3 mit $50 \mathrm{~km} / \mathrm{h}$. Zwischen den Abbildungen vergeht eine Zeitspanne von 0,5 s. Die im jeweiligen Zeitschritt geplante Trajektorie und das Umfeldmodell sind in grau, die resultierende Trajektorie ist in schwarz dargestellt. 
In Abbildung 6.7 sind die zugehörigen dynamischen Größen während des Manövers dargestellt. Die Beschleunigungsebene zeigt die Kritikalität, da das Beschleunigungspotential des Fahrzeugs ausgeschöpft wird. Der Solllenkradwinkel und -schlupf zeigen teilweise nicht realisierbare Signalverläufe, was aber im Sinne einer Übererregung die entsprechenden Istgrößen stärker beeinflusst.
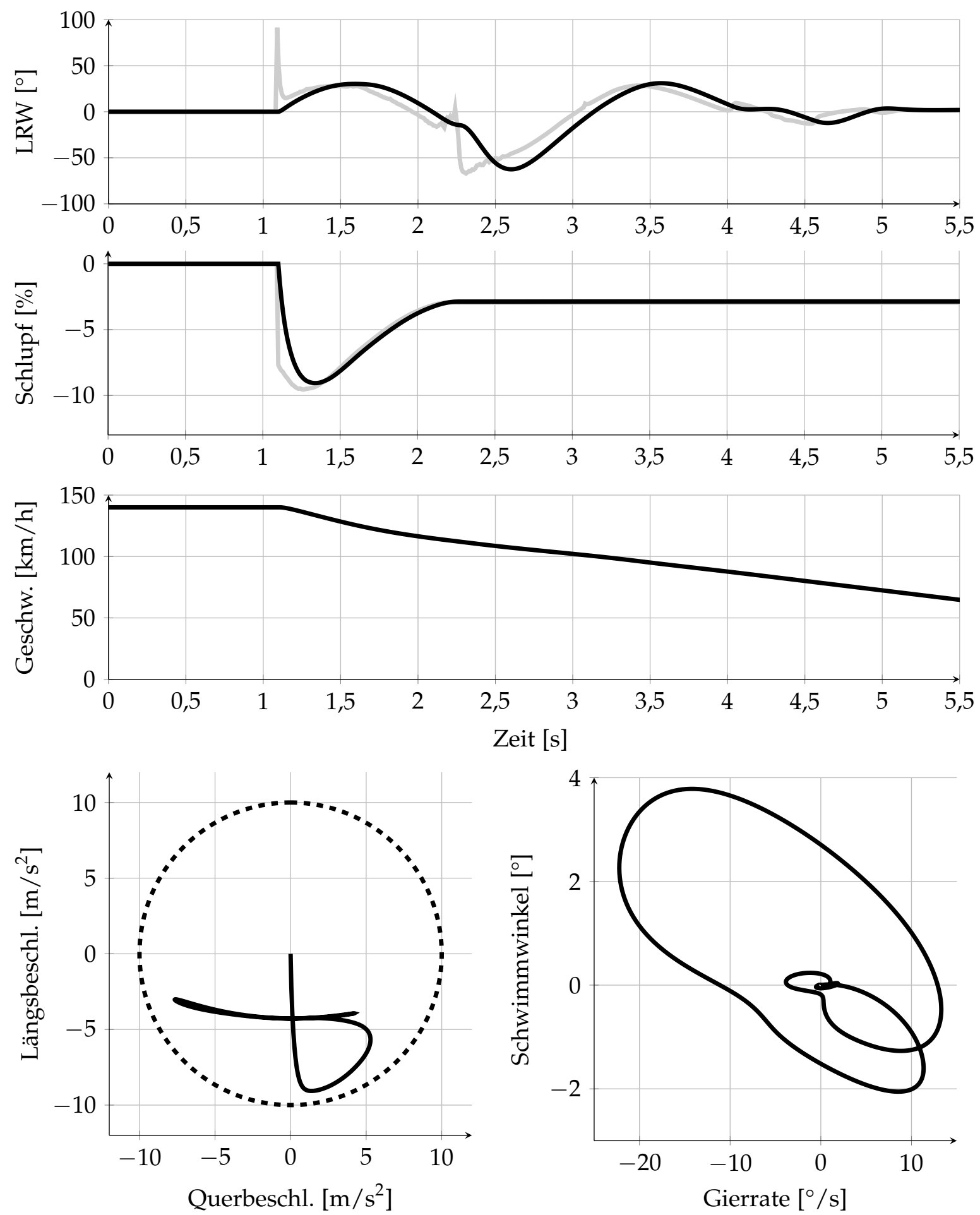

Abbildung 6.7.: Dynamische Größen während eines Kollisionsvermeidungsmanövers mit sich bewegenden Hindernissen bei einer initialen Geschwindigkeit von $v_{\text {init }}=140 \mathrm{~km} / \mathrm{h}$. Sollgrößen sind in grau, Istgrößen in schwarz gezeichnet. 


\section{7}

\section{Modellprädiktives Trajektorienscharverfahren}

Die Verfahren OTO und MPPC sind variabel einsetzbar, über das Gütemaß anpassbar und lösen viele verschiedene Verkehrssituationen. Jedoch sind sie aufwändig und die Wahl von Gewichten, um eine bestimmtes Verhalten zu erzeugen, nicht immer trivial. Der Rechenaufwand ist hoch und die Konvergenz innerhalb einer beschränkten Anzahl von Optimierungsiterationen nicht gesichert. Die Idee der modellprädiktiven Planung und Regelung bietet grundlegende Vorteile, welche in einem einfacheren Verfahren, dem modellprädiktiven Trajektorienscharverfahren, genutzt werden sollen. Das MPTSA liefert zwar nur eine suboptimale Lösung des Optimalsteuerungsproblems, benötigt jedoch keine rechenintensive iterative Optimierung. Anders als beim MPPC bietet die Erhöhung des Prädiktionshorizontes über einem bestimmten Wert hinaus aufgrund der konstanten Stellgrößen keine Verbesserung. Als Folge können weiter entfernte Hindernisse nicht bei der Planung berücksichtigt werden. Dieser Nachteil muss aber vor dem Hintergrund der limitierten Reichweite der Umfeldsensoren bewertet werden (siehe Kapitel 8). Die Vorteile sind die hohe Genauigkeit durch das dynamische Prädiktionsmodell und die Planung und Regelung in einem Verfahren, wodurch die unterlagerten Regler nicht benötigt werden und eine hohe Regelungsgüte erreicht wird. Im Grundsatz bleibt die in Abbildung 7.1 gezeigte Systemarchitektur des MPPC erhalten.

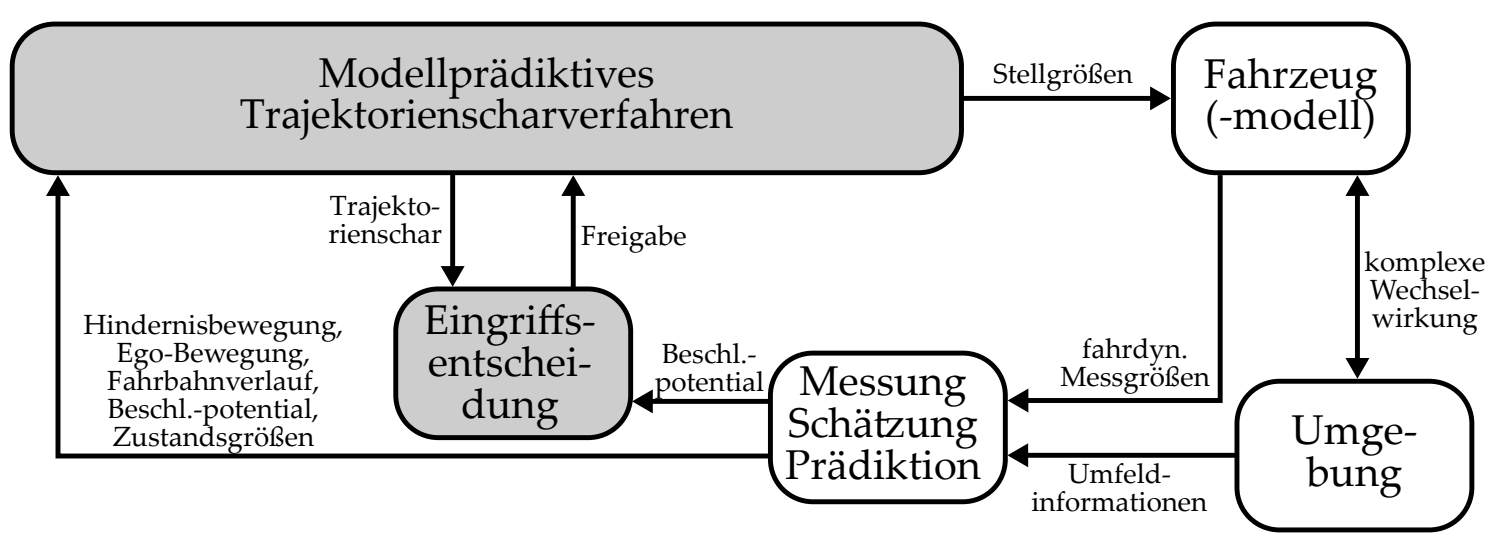

Abbildung 7.1.: Architektur des modellprädiktiven Trajektorienscharverfahrens. 


\subsection{Trajektorienschar und suboptimale Lösung des Optimalsteuerungsproblems}

Die Vereinfachung gegenüber dem MPPC wird durch eine Diskretisierung der Stellgrößen erreicht. Der Kontrollhorizont wird auf $n_{c}=1$ beschränkt und der Prädiktionshorizont $n_{p}>1$ gewählt, d.h. die Stellgrößen werden auf dem Prädiktionshorizont konstant gehalten. Die möglichen Werte, welche die Stellgrößen annehmen können, werden entweder grob diskretisiert oder es wird eine adaptive Stellgrößendiskretisierung verwendet, welche im Abschnitt7.2 diskutiert wird. Im Falle der festen Diskretisierung haben sich die beiden Mengen

$$
\begin{gathered}
\delta_{\text {soll }} \in A:=\left\{-360^{\circ} ;-225^{\circ} ;-135^{\circ} ;-75^{\circ} ;-30^{\circ} ;-15^{\circ} ;-5^{\circ} ; 0^{\circ} ; \ldots\right. \\
\left.\ldots 5^{\circ} ; 15^{\circ} ; 30^{\circ} ; 75^{\circ} ; 135^{\circ} ; 225^{\circ} ; 360^{\circ}\right\} \text { und } \\
s_{\text {soll }} \in B:=\{0 ;-0,04 ;-0,08 ;-0,12\},
\end{gathered}
$$

für alle betrachteten Geschwindigkeiten und für gerade Fahrbahnen als geeignet erwiesen (siehe $[\overline{K e l+15 a}])$. Durch die Einschränkung der möglichen Stellfolgen wird eine suboptimale Lösung des Optimalsteuerungsproblems gefunden. Es ist aber dadurch möglich auf einem gängigen Echtzeitsystem (dSPACE) für alle möglichen Kombinationen aus den Mengen der Stellgrößen die Lösung der Zustandsdifferentialgleichungen in Echtzeit zu bestimmen. Die benötigte Rechenzeit ist darüber hinaus in jedem Abtastschritt identisch und kann deterministisch vorhergesagt werden. Bei der iterativen Optimierung der Verfahren aus den Kapiteln 5 und 6 ist dies nicht möglich. Die Prädiktion der Bewegungstrajektorien gleicht bei Erregung aus der Ruhelage einem symmetrischen Fächer, welcher in Abbildung 7.2 exemplarisch skizziert ist. Das Sche-

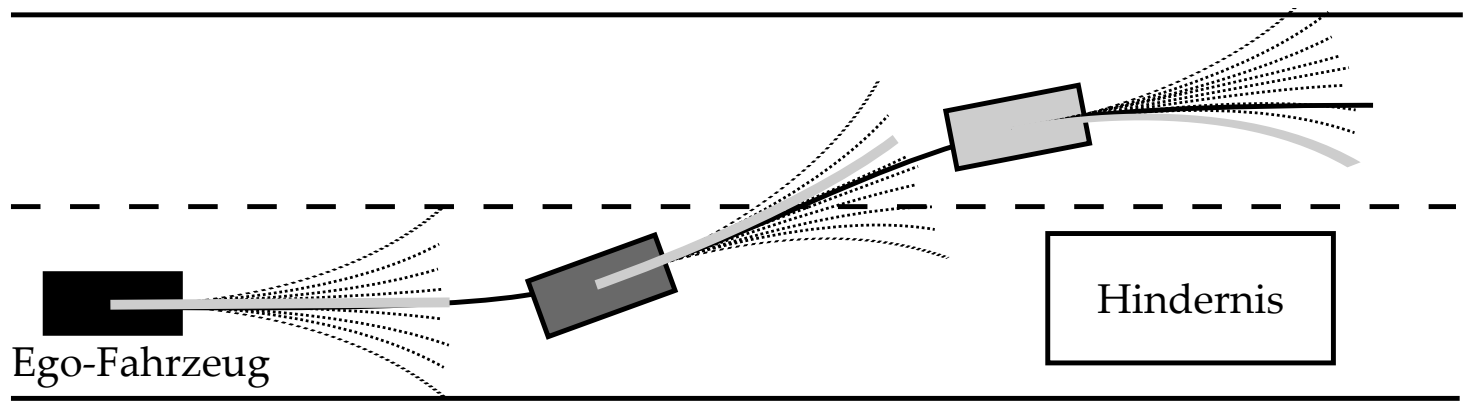

Abbildung 7.2.: Exemplarische Darstellung des Trajektorienscharverfahrens. Das Ego-Fahrzeug wird exemplarisch an drei verschiedenen Zeitpunkten gezeigt. Die optimale Trajektorie ist hellgrau und der restliche Fächer schwarz gepunktet dargestellt. Die resultierende Trajektorie ist schwarz und durchgezogen gezeichnet.

ma des Verfahrens ist in Abbildung 7.3 dargestellt. In jedem Abtastschritt werden, beginnend vom aktuellen Startzustand $\mathbf{x}_{0}$, die Zustandsdifferentialgleichungen des Prädiktionsmodells gelöst. Aus diesen möglichen Trajektorien wird diejenige ausgewählt, welche den geringsten Wert eines Gütemaßes JMPTSA liefert, dessen Aufbau in Abschnitt 7.3 erläutert wird. Die zugehörigen Stellgrößen werden auf die Regelstrecke angewendet. Das Verfahren weist eine große Ähnlichkeit mit der modellprädikti- 


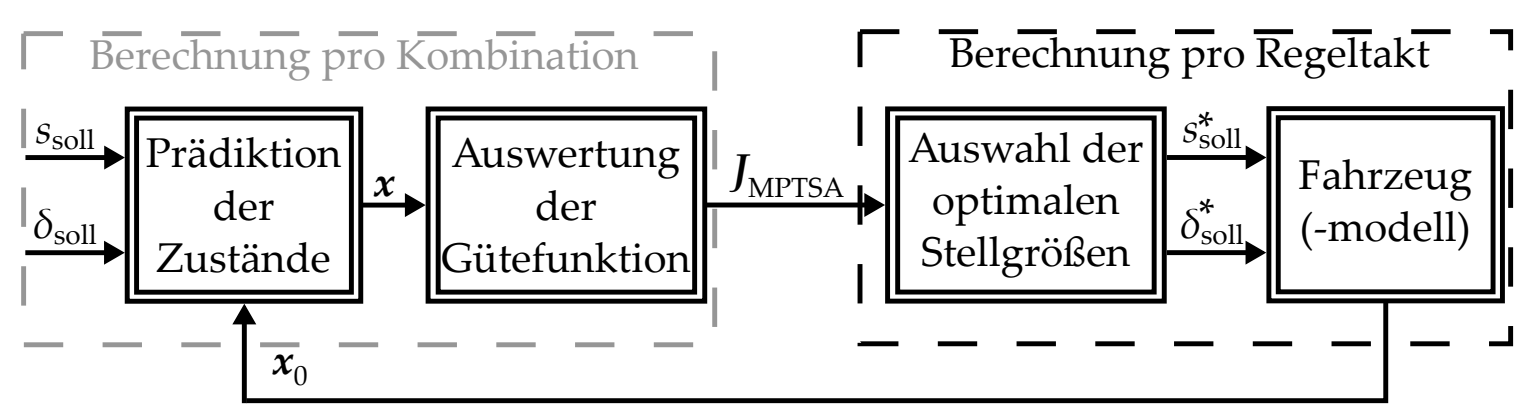

Abbildung 7.3.: Blockschaltbild des modellprädiktiven Trajektorienscharverfahrens.

ven Regelung auf. Aufgrund der Stellgrößendiskretisierung ergibt sich jedoch eine suboptimale Lösung des Optimalsteuerungsproblems

$$
(\mathbf{x}, \mathbf{u})^{*}=\arg \min _{\mathbf{u}} J_{\text {MPTSA }}
$$

unter der Nebenbedingung, dass die optimalen Stellgrößen aus der Menge der diskreten Stellgrößen

$$
\mathbf{u} \in(A \times B)
$$

bestehen und die Zustandstrajektorien sich aus der Lösung des Prädiktionsmodells

$$
\mathbf{x}_{k+1}=\mathbf{x}_{k}+\mathbf{f}\left(\mathbf{x}_{k}, \mathbf{u}_{k}\right) \Delta t, \mathbf{x}_{k=0}=\mathbf{x}_{0}
$$

ergeben. Hierin ist $\mathbf{x}_{0}$ der aktuell gemessene bzw. rekonstruierte Zustandsvektor.

\subsection{Adaptive Stellgrößendiskretisierung}

Eine feste Diskretisierung erlaubt es im stationären Fall nur bestimmten Krümmungen exakt zu folgen. Wenn z.B. einer Autobahnabfahrt mit konstanter Krümmung gefolgt werden soll, wird das MPTSA häufig zwischen zwei Sollwerten hin und her schalten. Eine genauere Diskretisierung des gesamten Stellbereichs führt auf einen zu hohen Rechenaufwand, sodass die Berechnung in Echtzeit nicht mehr möglich ist. Häufig ist eine hohe Auflösung nur in einem bestimmten Bereich erforderlich, welcher sich jedoch mit der Zeit verschieben kann. Im Folgenden wird daher gezeigt, wie durch eine adaptive Diskretisierung die möglichen Nachteile einer festen Diskretisierung vermieden werden können. Es wird ein Polynom 4. Grades angesetzt, um eine äquidistante (oder lineare) Diskretisierung auf eine adaptive abzubilden. Das Polynom lautet

$$
\delta_{\mathrm{aD}}\left(\delta_{\mathrm{lD}}\right)=a D_{4} \delta_{\mathrm{lD}}^{4}+a D_{3} \delta_{\mathrm{ID}}^{3}+a D_{2} \delta_{\mathrm{ID}}^{2}+a D_{1} \delta_{\mathrm{ID}}+a D_{0}
$$

und wird durch die Randbedingungen

$$
\begin{gathered}
\delta_{\mathrm{aD}}\left(\delta_{\text {max }, \mathrm{dis}}\right)=\delta_{\text {max }, \mathrm{dis}}, \delta_{\mathrm{aD}}\left(-\delta_{\text {max }, \mathrm{dis}}\right)=-\delta_{\text {max }, \mathrm{dis}}, \delta_{\mathrm{aD}}(0)=\delta_{\text {soll }, k-1}, \\
\frac{\mathrm{d} \delta_{\mathrm{aD}}(0)}{\mathrm{d} \delta_{\mathrm{lD}}}=0 \text { und } \frac{\mathrm{d}^{2} \delta_{\mathrm{aD}}(0)}{\mathrm{d} \delta_{\mathrm{lD}}^{2}}=0
\end{gathered}
$$


eindeutig bestimmt. Aus den Bedingungen ergibt sich ein lineares Gleichungssystem, dessen Lösung die Koeffizienten

$$
\begin{gathered}
a D_{0}=\delta_{\text {soll }, k-1}, a D_{1}=0, a D_{2}=0, \\
a D_{3}=\frac{1}{\delta_{\text {max,dis }}^{2}} \text { und } a D_{4}=-\frac{\delta_{\text {soll }, k-1}}{\delta_{\text {max,dis }}^{4}}
\end{gathered}
$$

sind. Die ersten beiden Randbedingungen sollen die adaptive Diskretisierung auf den erlaubten Stellbereich beschränken. Die anderen Bedingungen erzeugen einen Sattelpunkt an der Stelle des im letzten Abtastschrittes gewählten Sollwertes $\delta_{\text {soll }, k-1}$. Dadurch wird in dem Bereich um den letzten Sollwert eine höhere Auflösung erreicht, was in Abbildung 7.4 zu sehen ist. Der Verlauf für einen letzten Sollwert nahe der

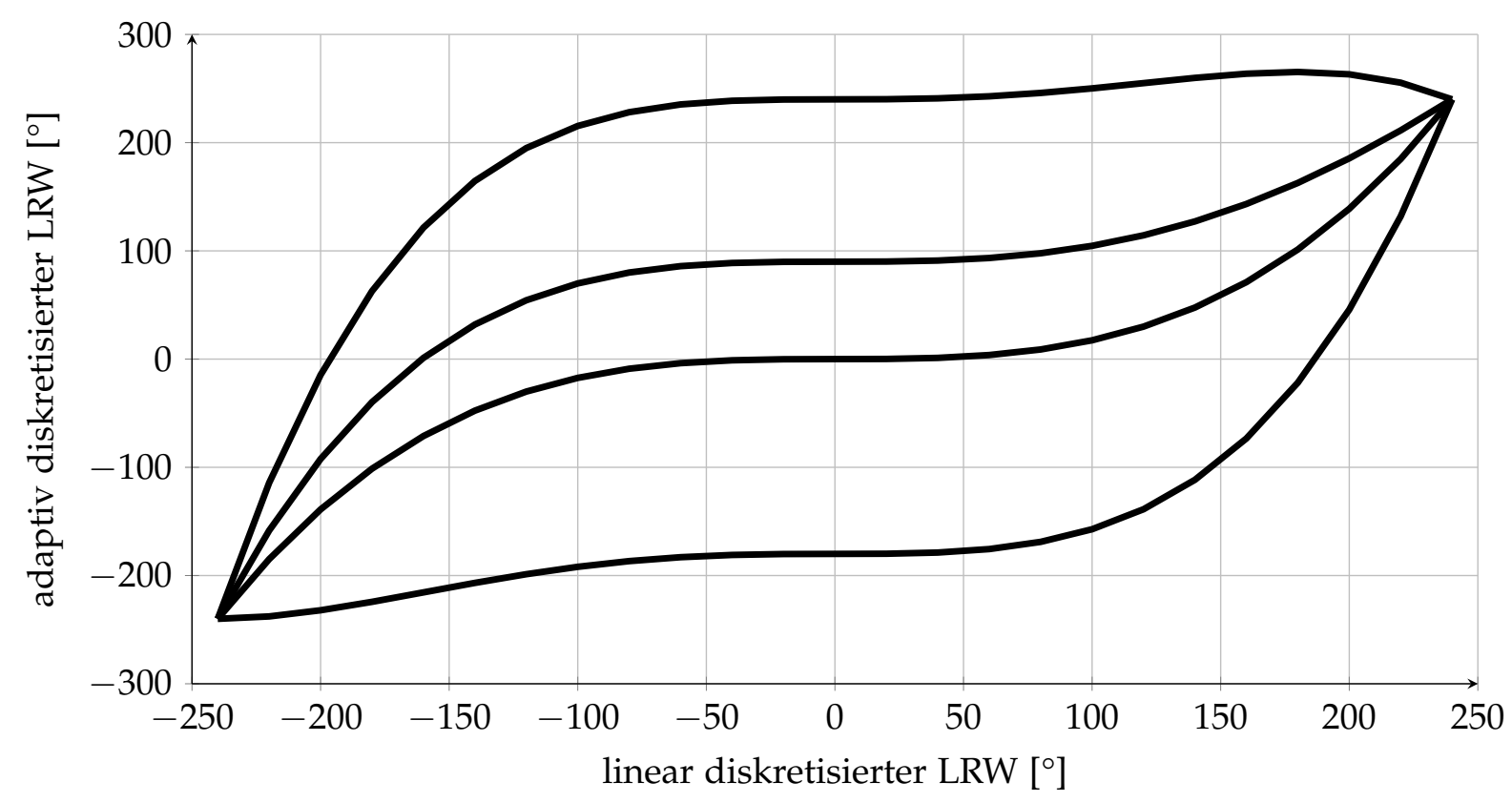

Abbildung 7.4.: Abbildung der linearen auf die adaptive Stellgrößendiskretisierung für unterschiedliche letzte Sollwerte (von oben nach unten): $\delta_{\text {soll }, k-1}=240^{\circ}, \delta_{\text {soll }, k-1}=90^{\circ}, \delta_{\text {soll }, k-1}=0^{\circ}$, $\delta_{\text {soll }, k-1}=-180^{\circ}$.

Stellgrößenbeschränkung geht über diese hinaus. Dies ist jedoch kein wesentlicher Nachteil, da der Verlauf nachträglich auf den erlaubten Stellbereich beschränkt wird.

\subsection{Entwurf des Gütemaßes zur Kollisionsvermeidung}

\subsubsection{Aufbau des Gütemaßes}

Das Umfeldmodell aus Kapitel 5 wurde entsprechend den Anforderungen einer iterativen Minimierung gestaltet. Zudem wurde darauf geachtet, dass durch Hindernisse keine lokalen Minima entstehen. Bei dem hier betrachteten Verfahren ist dies nicht notwendig. Um Kollisionen auf dem Prädiktionshorizont mit Hindernissen und Fahrbahnbegrenzungen festzustellen, wird die Lage der vier Eckpunkte des Ego-Fahrzeugs 
betrachtet. Es liegt eine Kollision vor, wenn einer der Eckpunkte die Fahrbahnbegrenzung überschreitet oder der Abstand zum Schwerpunkt eines Hindernisses $d_{\text {obs }}$ kleiner wird als der Grenzwert

$$
d_{\text {Limit }}=d_{1}+d_{2} \cos ^{f_{\mathrm{p}}}(\Phi) \text {. }
$$

Hierin sind $d_{1}$ und $d_{2}$ Abstandsmaße, $\Phi$ der relative Gierwinkel zwischen dem Egound dem Hindernisfahrzeug und der Exponent $f_{\mathrm{p}}$ ein Formfaktor. Die Gleichung ist mit weniger Rechenaufwand auswertbar als die Überprüfung, ob einer der Eckpunkte innerhalb des vom Hindernis aufgespannten Rechtecks liegt. In Abbildung 7.5 ist Gleichung (7.3.1) graphisch in der Draufsicht dargestellt. Durch die geeignete Wahl

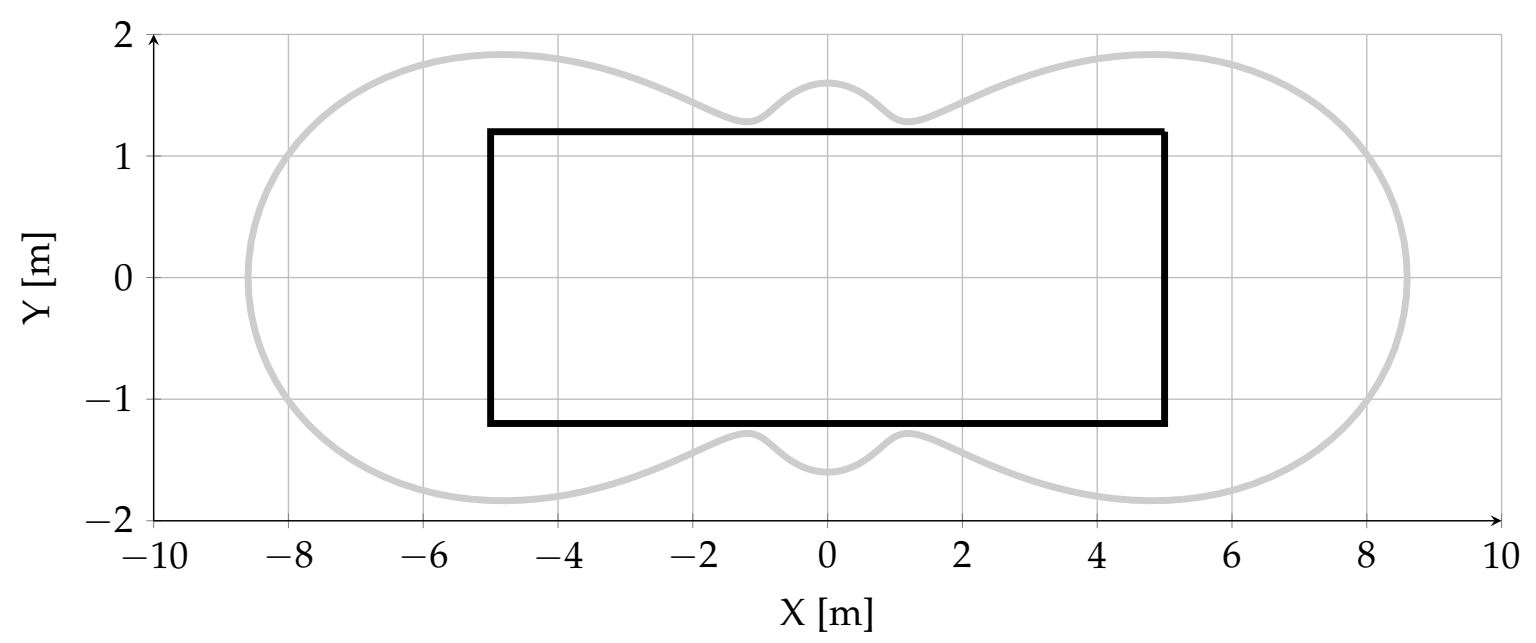

Abbildung 7.5.: Approximation der Fahrzeugform durch eine Sicherheitsblase.

von $d_{1}, d_{2}$ und $f_{\mathrm{p}}$ wird die rechteckige Form des Hindernisses approximiert. Zudem sind die Sicherheitsabstände vor und hinter dem Hindernis größer als seitlich daneben, was dem Gefahrenniveau dieser Gebiete Rechnung trägt. Die Anforderungen an das Gütemaß aus funktionaler Sicht sind beim MPTSA identisch zu denen des OTO oder des MPPC. Es besteht jedoch die Besonderheit, dass das Gütemaß nicht etwa stetig differenzierbar sein muss. Vielmehr können beliebige Terme, wie z.B. logische Ausdrücke, verwendet werden. Die Gewichtungen der einzelnen Gütemaßanteile sollte mit einfachen Heuristiken zu wählen sein und möglichst keinen Einfluss auf das Verhältnis zwischen Längs- und Querführung haben. Ein Gütemaß, welches den Anforderungen genügt, lautet:

$$
J_{\text {MPTSA }}=\sum_{k=1}^{n_{\mathrm{p}}}\left(\gamma_{\Psi} \Psi_{k}^{2}+\gamma_{\mathrm{env}} \Gamma_{\mathrm{pf}, k}+\gamma_{\mathrm{s}} \mathrm{s}_{\mathrm{soll}, k}^{2}\right)
$$

mit

$$
\Gamma_{\mathrm{pf}, k}= \begin{cases}2^{\left(n_{\mathrm{p}}-k\right)} & \text { wenn } d_{\mathrm{obs}}<d_{\text {Limit }} \\ 0 & \text { sonst. }\end{cases}
$$

\subsubsection{Wahl der Gewichtungsfaktoren}

Das Gütemaß beinhaltet die drei Anteile: Minimierung des Gierwinkels, Kollisionsfreiheit und Minimierung des Bremseingriffs (Energieoptimalität). Die Bestrafung 
des Gierwinkels minimiert indirekt die fahrdynamischen Reaktionen. Die zusätzliche Bestrafung des Schwimmwinkels und/oder der Gierrate wie beim MPPC hat sich beim MPTSA als unnötig erwiesen, da ein instabiler Fahrzustand mit einem größeren Gierwinkel einhergeht und auch aufgrund der auf dem Prädiktionshorizont konstanten Stellgrößen, welche extrem gekrümmte Trajektorien verhindern. Der zweite Anteil bestraft Kollisionen auf dem Prädiktionshorizont, wobei die Gleichung (7.3.3) Kollisionen in ferner Zukunft weniger stark bestraft als solche in naher Zukunft. Diese Wahl bewirkt, dass eine einzelne Kollision zum Zeitpunkt $k$ einen höheren Beitrag zum Gütemaß bewirkt als alle Kollisionen ab dem Punkt $k+1$ zusammen. Der dritte Anteil minimiert die längsdynamischen Eingriffe, wobei das Gewicht $\gamma_{\mathrm{s}}$ adaptiv gewählt wird. Die Zustandsdifferentialgleichungen des Prädiktionsmodells werden auf dem Prädiktionshorizont $T_{\text {pr }}$ gelöst und bilden die Schar der möglichen Lösungen. Zusätzlich werden die Bewegungsgleichungen auch für das freie System $\mathbf{u}=\mathbf{0}$ und für die aktuell durch den Fahrer bzw. das System gewählten Zustandsgrößen $\mathbf{u}=\left(\begin{array}{ll}\delta & s_{1}\end{array}\right)^{\mathrm{T}}$ über einen kürzeren Horizont $T_{\mathrm{Kol}}<T_{\mathrm{pr}}$ gelöst. Es wird $\gamma_{\mathrm{s}}=0$ gewählt, wenn

- für das freie System oder die aktuellen Stellgrößen eine Kollision prädiziert wird oder

- die fahrdynamischen Zustandsgrößen Grenzwerte überschritten haben:

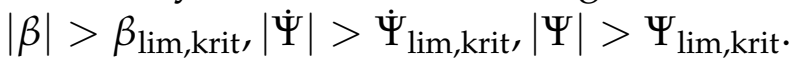

Durch diese Wahl werden Bremseingriffe und Lenkeingriffe gleichermaßen nicht bestraft. Wenn Bremseingriffe nicht benötigt werden, wird $\gamma_{\mathrm{s}}>>1$ gewählt. Durch die heuristische Wahl $\gamma_{\mathrm{env}}>>\gamma_{\Psi}$ wird der Kollisionsvermeidung eine deutlich höhere Bedeutung zugemessen als der Minimierung des Gierwinkels. Diese Wahl und die Bewertung der Kollisionen in Abhängigkeit der Zeit nach Gleichung (7.3.3) bewirken, dass die Trajektorie mit dem minimalen Gierwinkel im quadratischen Mittel ausgewählt wird, solange kollisionsfreie Trajektorien prädiziert werden. Tritt bei allen Trajektorien aus der Schar eine Kollision auf, so wird diejenige gewählt, welche zeitlich am längsten kollisionsfrei ist. Dieses Auswahlverhalten ließe sich auch auf einem anderen Wege erzielen, jedoch zeigt die Formulierung in einem Gütemaß die Verwandtschaft zum MPPC und die modellprädiktiven Eigenschaften des MPTSA auf. Zusammenfassend gilt die folgende Heuristik zur Wahl der Gewichte:

- Wenn kein Bremseingriff benötigt wird gilt: $\gamma_{\Psi}<<\gamma_{s}<<\gamma_{\mathrm{env}}$,

- sonst gilt: $\gamma_{\Psi}<<\gamma_{\mathrm{env}}, \gamma_{\mathrm{s}}=0$.

\subsubsection{Kritikalitätsmaß und Eingriffsentscheidung}

Als modellprädiktives Verfahren bietet das MPTSA ähnlich wie das MPPC die Möglichkeit mehrere Bewegungspotentiale zu betrachten. Der kurze Prädiktionshorizont bietet jedoch eine eingeschränkte Bewertungsmöglichkeit, da zu Beginn eines Notmanövers die Zustands- und Stellgrößen am Ende des Manövers nicht abgeschätzt werden können. Für eine bessere Vergleichbarkeit mit dem OTO wird die Kritikalität zur Eingriffsentscheidung auch beim MPTSA über den Nutzungsgrad des Beschleunigungspotentials nach Gleichung (5.2.10) approximiert. 


\subsection{Ergebnisse}

Die im Folgenden gezeigten Simulationsergebnisse wurden mit einer adaptiven Diskretisierung des Solllenkradwinkels und einer festen Diskretisierung des Sollschlupfes gewonnen. Abbildung 7.6 zeigt die Trajektorienschar in einer beispielhaften Verkehrssituation. Der querdynamische Zustand des Ego-Fahrzeugs entspricht in dieser Situation der Ruhelage $(\beta=0, \dot{\Psi}=0)$. Aufgrund der adaptiven Diskretisierung ist der Fächer der Bewegungstrajektorien nicht symmetrisch, da im vorherigen Abtastschritt ein von Null verschiedener Lenkradwinkel gewählt wurde. Analog zum OTO und

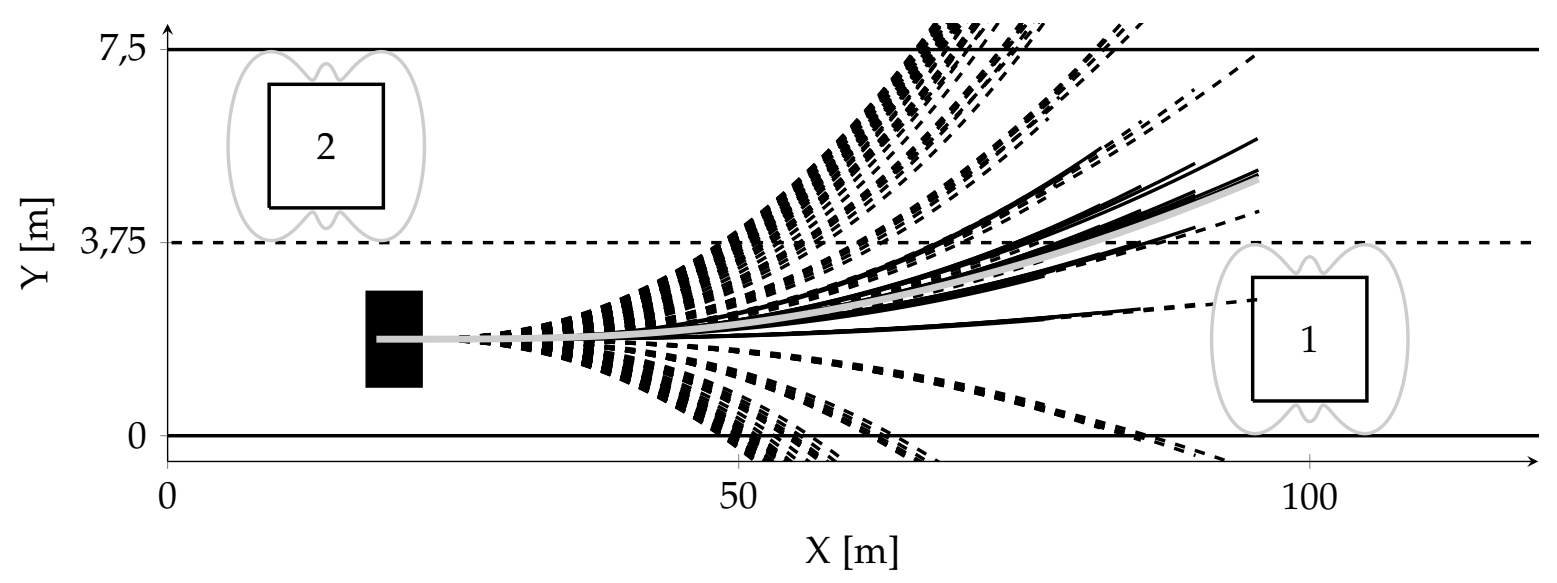

Abbildung 7.6.: Trajektorienschar in einer Beispielsituation. Das Ego-Fahrzeug wird durch das ausgefüllte Rechteck dargestellt. Kollisionsbehaftete Trajektorien sind in schwarz und gestrichelt, kollisionsfreie in schwarz und durchgezogen, die ausgewählte Trajektorie in hellgrau und durchgezogen dargestellt. Die Hindernisse sind als nicht ausgefüllte Rechtecke mit Approximation durch das Umfeldmodell dargestellt.

MPPC werden die gleichen Verkehrssituationen untersucht. Die Ergebnisse sind in Abbildung 7.7 dargestellt. Wie zu erwarten ist, hält das Ego-Fahrzeug bei einer initialen Geschwindigkeit von $60 \mathrm{~km} / \mathrm{h}$ vor dem ersten Hindernis an, was in Abbildung 7.7 oben zu erkennen ist. Dabei wird das Längsbeschleunigungspotential des Fahrzeugs vollkommen ausgenutzt. Bei einer Geschwindigkeit von $100 \mathrm{~km} / \mathrm{h}$ führt auch das MPTSA ein kombiniertes Ausweichmanöver durch und hält vor dem zweiten Hindernis an, wie Abbildung 7.7 mittig zeigt. Dies weicht in dieser Situation vom Ergebnis des OTO ab und entspricht der Lösung durch das MPPC. Auch bei diesem Manöver wird das Längsbeschleunigungspotential voll ausgeschöpft. Dennoch bleibt die Fahrzeugführung stabil. Abbildung 7.7] unten zeigt das Ergebnis bei einer Geschwindigkeit von $v_{\text {init }}=140 \mathrm{~km} / \mathrm{h}$, welches dem mit dem MPPC erzielten Ergebnis prinzipiell gleicht. Zunächst wird ein Doppelspurwechsel durchgeführt, um den Hindernissen 1 und 2 auszuweichen, bevor anschließend das Ego-Fahrzeug vor den Hindernissen 3 und 4 anhält. Insgesamt ist zu erkennen, dass die Querführung durch das MPTSA eine ähnliche Präzision beinhaltet wie beim MPPC. Der Fahrschlauch, welcher durch die ausgefüllten Rechtecke dargestellt wird, berührt die Grenzlinien (grau) des Umfeldmodells, schneidet diese jedoch nicht. Es ist zu beachten, dass sich die Grenzen des Umfeldmodells auf die Ecken des Fahrzeugaufbaus beziehen und nicht auf den Schwerpunkt, wie es beim OTO und MPPC der Fall ist. Im Vergleich zum 

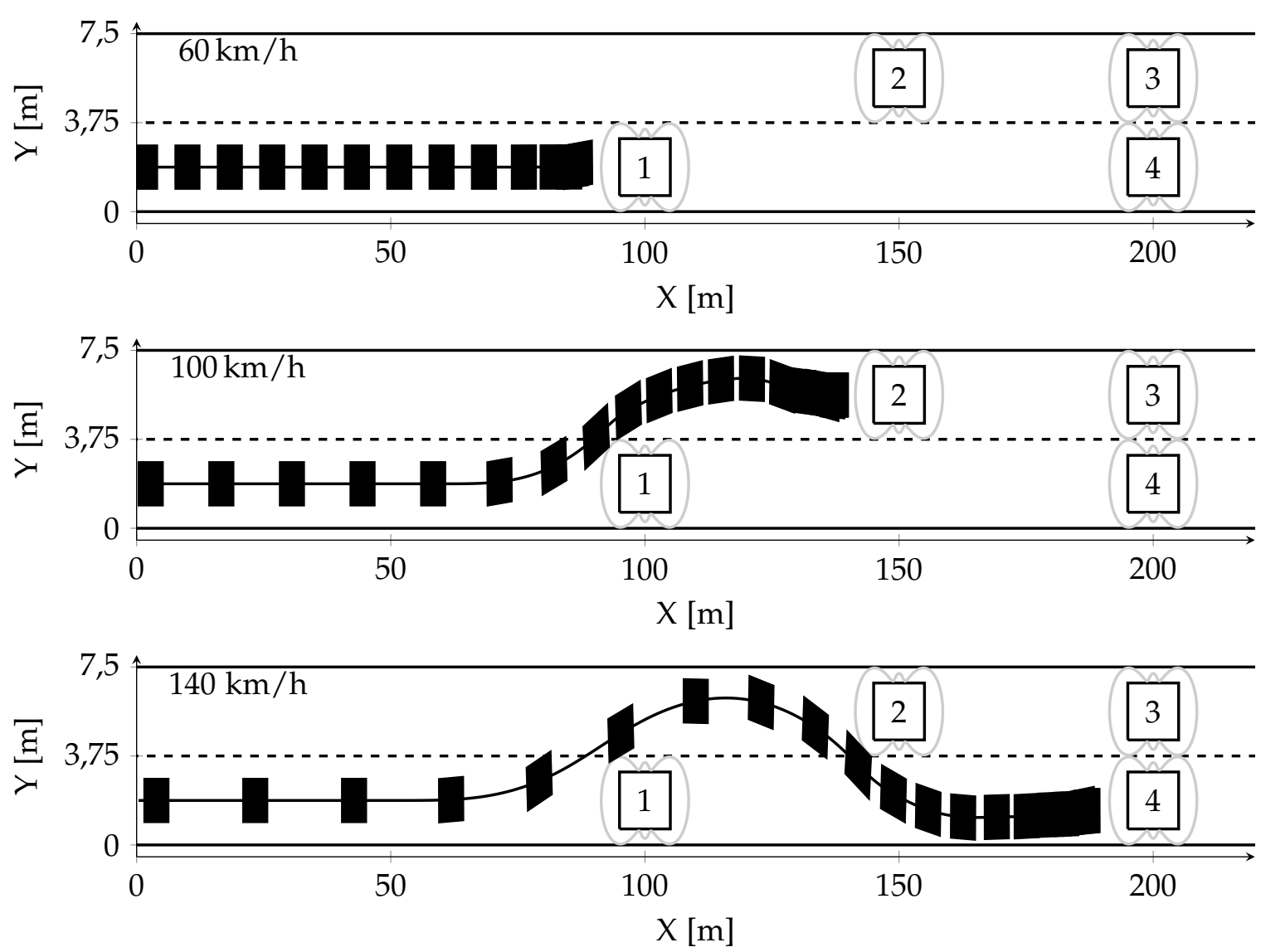

Abbildung 7.7.: Ergebnis bei den initialen Geschwindigkeiten $v_{\text {init }}=60,100$ und $140 \mathrm{~km} / \mathrm{h}$. Das Ego-Fahrzeug wird durch schwarz ausgefüllte Vierecke in Abständen von 0,5s, die Hindernisse durch schwarz gerahmte Rechtecke dargestellt. Das Umfeldmodell wird mit grauen Linien gezeichnet.

MPPC werden die Anhaltepunkte früher gewählt, was auf die fehlende Bestrafung des Sollschlupfes in entsprechenden Situationen zurückzuführen ist. Das MPPC nutzt den zur Verfügung stehenden Fahrkorridor besser aus. Auch die Verkehrssituation mit den dynamischen Hindernissen in Abbildung 7.8 wird kollisionsfrei gelöst. Sowohl die Beschleunigungen als auch der Schwimmwinkel und die Gierrate in Abbildung 7.9 zeigen, dass es sich dabei um ein aus fahrdynamischer Sicht schwieriges Manöver handelt. Trotzdem wird das Fahrzeug nach dem Manöver stabilisiert. Als letztes Ergebnis wird in Abbildung 7.10 gezeigt, wie das Verfahren auch externe Störungen, in diesem Fall durch den Fahrer im Fahrsimulator manuell eingeprägt, ausregelt. Hierzu wird die statische Stellgrößendiskretisierung verwendet. Die Verkehrssituation besteht aus einem stehenden Hindernis. Beim Einlenken auf die linke Fahrspur bringt der Fahrer zwischen $t=28,4 \mathrm{~s}$ und 28,9s einen unnötig großen Lenkradwinkel auf, wie es im Lenkradwinkelverlauf gekennzeichnet ist. Vor und nach der Störung greift der Fahrer nicht ein. Die Phasenebene zeigt, dass dies das Fahrzeug zum Übersteuern zwingt. Im Folgenden wird der instabile Fahrzustand ohne ESC und innerhalb der Fahrbahngrenzen ausgeregelt. Der Vergleich der Solllenkradwinkel in den Abbildungen 7.9 und 7.10 zeigt, dass sich bei der Verwendung der adaptiven Diskretisierung kleinere Sprünge in den Sollgrößen ergeben und somit eine genauere Regelung möglich wird. 

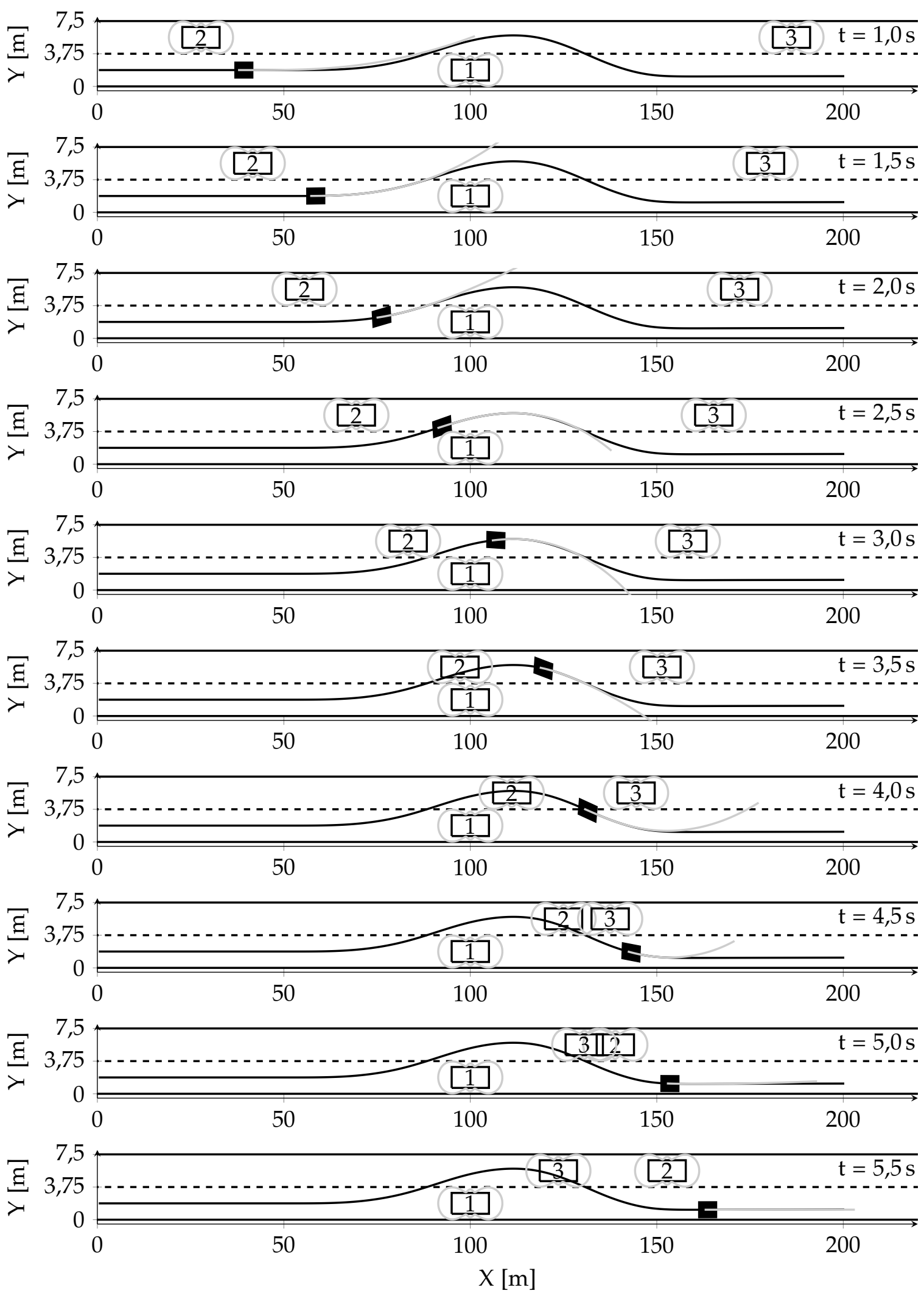

Abbildung 7.8.: Kollisionsvermeidungsmanöver mit einer initialen Geschwindigkeit von $v_{\text {init }}=140 \mathrm{~km} / \mathrm{h}$. Das nach rechts fahrende Hindernis 2 bewegt sich gleichförmig geradlinig mit $100 \mathrm{~km} / \mathrm{h}$, das nach links fahrende Fahrzeug 3 mit $50 \mathrm{~km} / \mathrm{h}$. Zwischen den Abbildungen vergeht eine Zeitspanne von 0,5 s. Die im jeweiligen Zeitschritt geplante Trajektorie und das Umfeldmodell sind in grau, die resultierende Trajektorie ist in schwarz dargestellt. 
Die Regelgrößen (Istgrößen) verlaufen aber in beiden Fällen ohne unnötige und unkomfortable Schwingungen, wie sie bei zu grober Diskretisierung entstehen (siehe $[\overline{K e l+15 c}])$. Die Verläufe des Soll- und Istschlupfes weisen im Vergleich zu den Verläufen des Lenkradwinkels eine größere Ungleichmäßigkeit auf, was aber aufgrund der Kritikalität des Manövers akzeptabel ist.
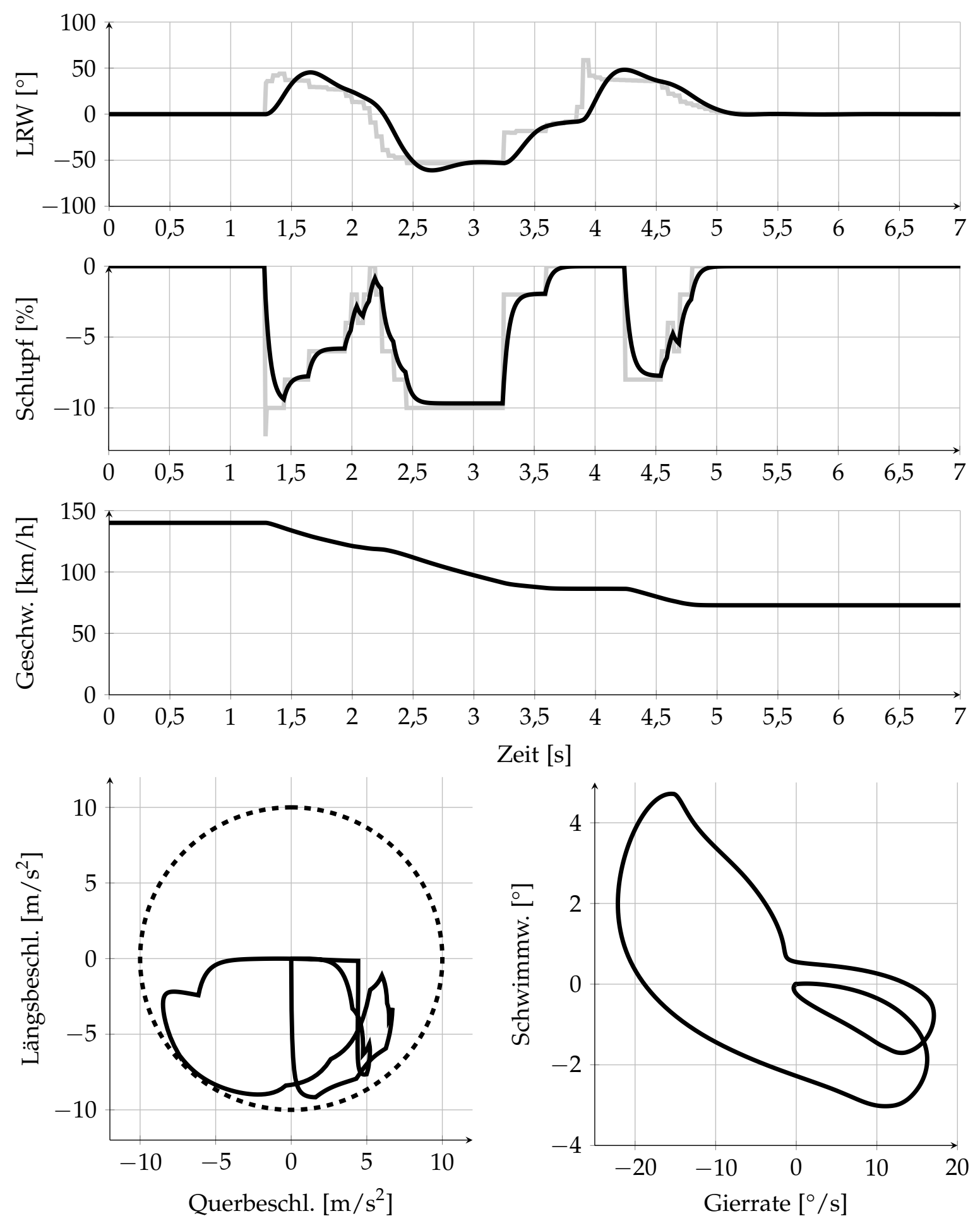

Abbildung 7.9.: Dynamische Größen bei dem Ausweichmanöver mit bewegten Hindernissen und eine initialen Geschwindigkeit des Ego-Fahrzeugs von $v_{\text {init }}=140 \mathrm{~km} / \mathrm{h}$. Sollgrößen sind grau und Istgrößen schwarz dargestellt. 

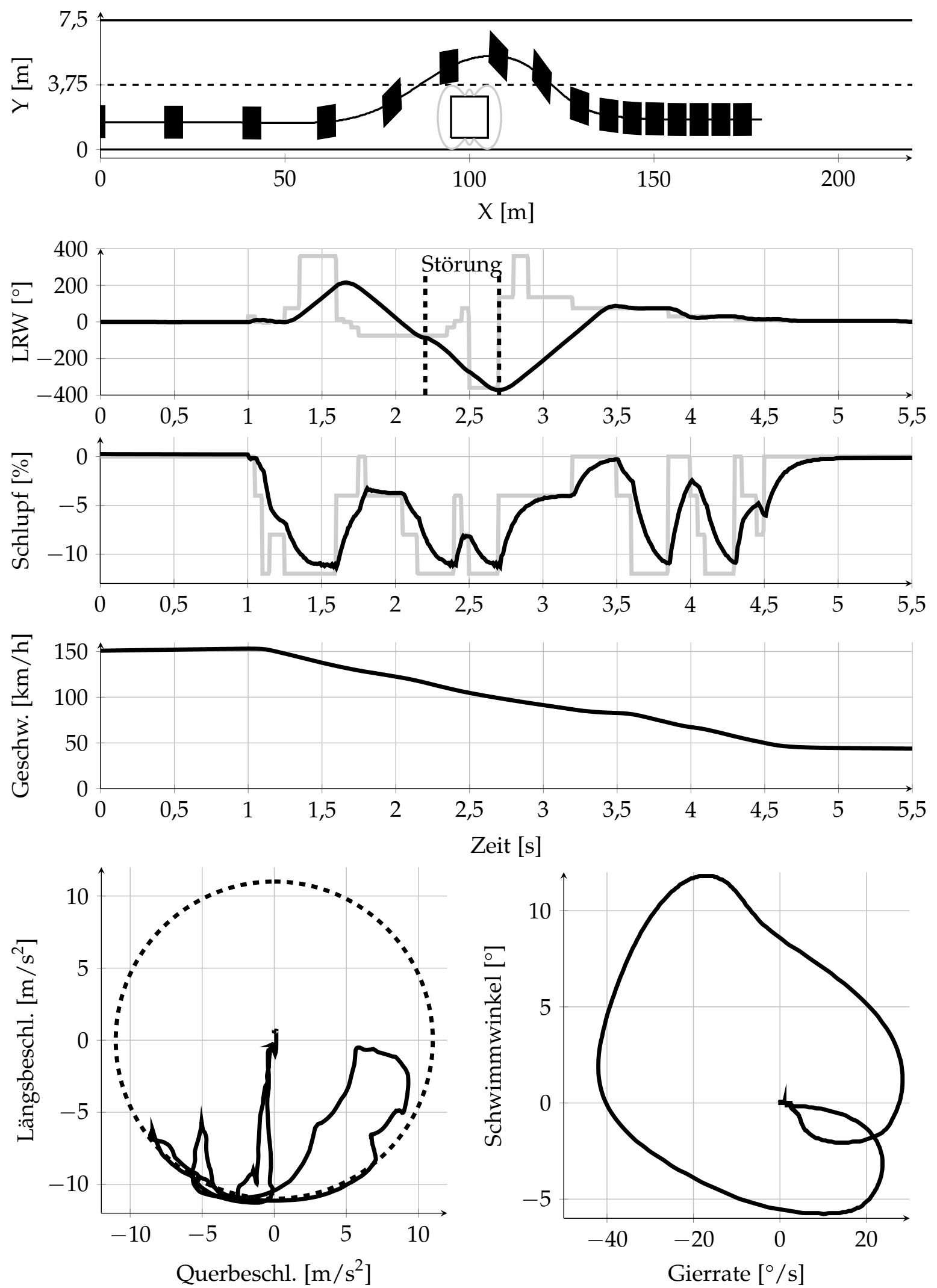

Abbildung 7.10.: Durch den Fahrer gestörtes Kollisionsvermeidungsmanöver bei $v_{\text {init }}=150 \mathrm{~km} / \mathrm{h}$ Startgeschwindigkeit. Das Ego-Fahrzeug ist in Abständen von 0,5 s abgebildet. Sollgrößen sind grau und Istgrößen schwarz dargestellt. 


\section{Analyse, Vergleich und Bewertung der Verfahren}

In diesem Kapitel werden zunächst Vergleichsmethoden diskutiert, darauf aufbauend eine Beispielsituation analysiert und der Vergleich mit der Nutzwertanalyse durchgeführt. Anschließend werden Modifikationen und Kombinationen der Verfahren ergänzend vorgestellt, bevor das Kapitel mit einem Fazit endet.

\subsection{Auswahl von Vergleichsmethoden}

Um die vier Verfahren zu vergleichen und zu bewerten, bietet es sich an, Gütekriterien zu definieren und diese für jedes der Verfahren in der gleichen Verkehrssituation auszuwerten. Als Verkehrssituation wird ein stehendes Hindernis auf der rechten Fahrspur ausgewählt, da diese Situation von allen Verfahren gelöst wird. Komplexere Situationen eignen sich für einen direkten Vergleich nur bedingt. Als Beispielsituation sei das Szenario aus den vorherigen Kapiteln mit vier stehenden Hindernissen angenommen. Die Kollisionsvermeidung mit dem zweiten Hindernis ist abhängig davon, wie die Kollision mit dem ersten Hindernis vermieden wurde. Da sich das Regelungsverhalten der Verfahren unterscheidet, wird das Manöver zur Kollisionsvermeidung mit dem zweiten Hindernis von unterschiedlichen Ausgangssituationen bestimmt und ist damit nicht vergleichbar. Als Gütekriterien bieten sich der minimale Abstand der Schwerpunktstrajektorie zur hinteren linken Hindernisecke und die Maximalwerte der fahrdynamischen Zustandsgrößen und der Beschleunigungen an. Diese Werte sind einfach zu bestimmen und erlauben eine objektive Bewertung. Der Nachteil dieser Methode ist, dass sie auf bestimmte Situationen beschränkt ist und von der konkreten Ausgestaltung der vier Verfahren in dieser Arbeit abhängt. Das Ziel der vorliegenden Arbeit ist aber darüber hinaus, die grundlegenden Eigenschaften und Unterschiede der Verfahren aufzuzeigen. Aus diesem Grund bietet sich die Nutzwertanalyse (auch Scoring-Modell genannt) an, welche einen systematischen Vergleich bei schwierig messbaren bzw. nicht messbaren Kriterien ermöglicht und somit zur Hervorhebung der grundlegenden Unterschiede zweckmäßig ist. 


\subsection{Vergleich in einer Beispielsituation}

In Abbildung 8.1 sind die Ergebnisse der vier Verfahren in der Standardsituation mit einer initialen Geschwindigkeit von $v_{\text {init }}=130 \mathrm{~km} / \mathrm{h}$ dargestellt. Zur besseren Vergleichbarkeit wird hier die Kritikalität mit der TTC bestimmt und bei einem Wert von $T T C=1,3 \mathrm{~s}$ ausgelöst. Alle Verfahren führen ein stabiles und kollisionsfreies Brems- und Lenkmanöver durch.
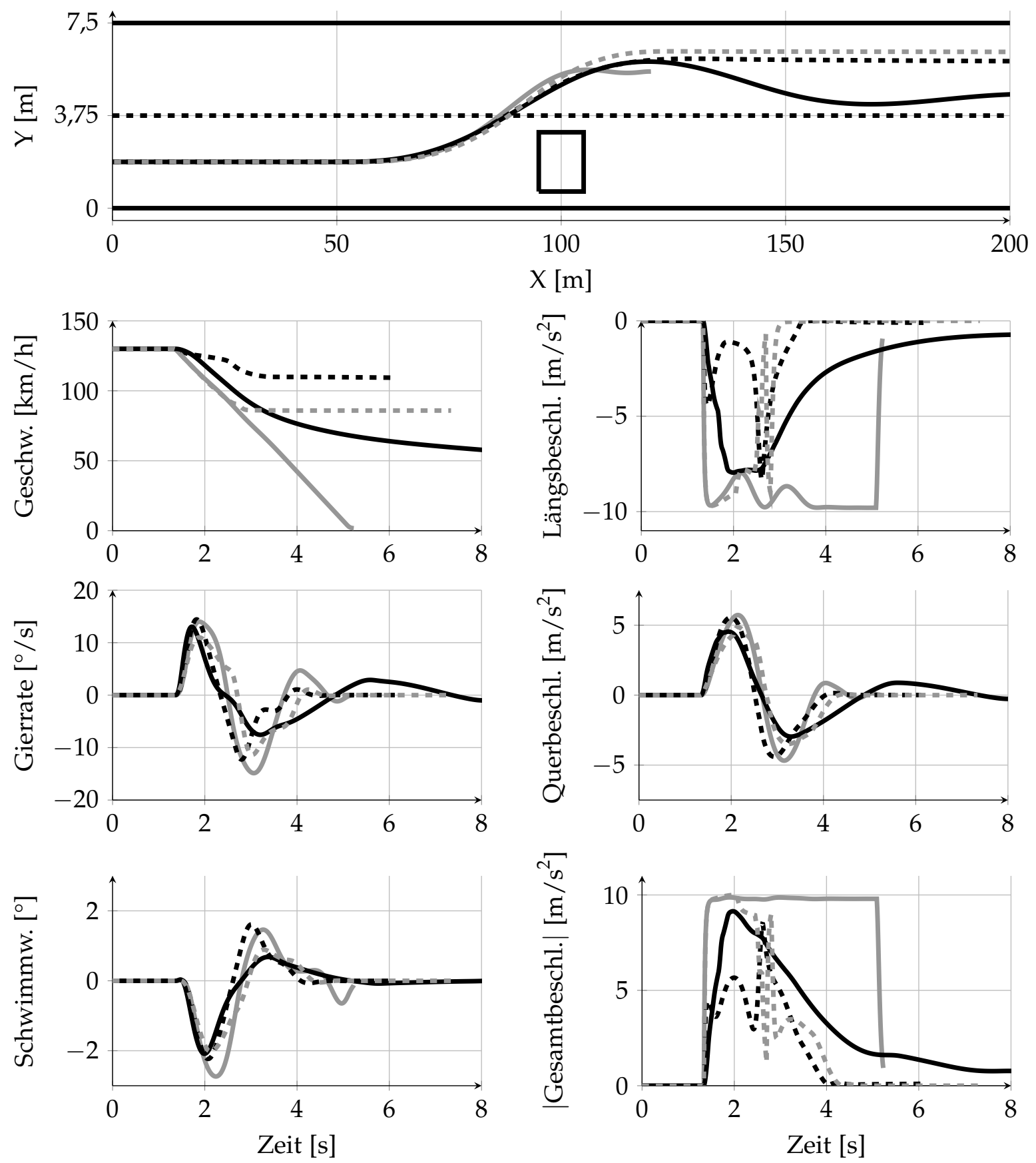

Abbildung 8.1.: Vergleich der vier Verfahren in einer Situation mit einem stehenden Hindernis. PFA: hellgrau, OTO: schwarz und durchgezogen, MPPC: schwarz und gestrichelt, MPTSA: grau und gestrichelt. Zur besseren Übersichtlichkeit sind die Umfeldmodelle und das Ego-Fahrzeug nicht dargestellt. 
Beim PFA kommt das Fahrzeug aufgrund der einfachen Längsregelungsstrategie ohne Fahrersteuerung hinter dem Hindernis zum Stehen. Dementsprechend tritt auch das höchste Gesamtbeschleunigungsniveau auf. Die drei anderen Verfahren führen das Fahrzeug am Hindernis vorbei und halten den Schwerpunkt in der linken Spur. Bei den modellprädiktiven Verfahren ergeben sich ähnlich geformte Bahnen, welche am Ende des Manövers nur einen geringen lateralen Abstand zueinander aufweisen. Anders als bei den Anhaltemanövern in den Kapiteln 6 und 7 nutzt hier das MPTSA den Fahrkorridor besser aus. Darüber hinaus fällt auf, dass das MPPC die geringste Geschwindigkeitsreduktion aufweist. Beim OTO tritt eine langsam abklingende Schwingung der querdynamischen Größen auf und die zur Kollisionsvermeidung aufgebaute Verzögerung wird nur langsam abgebaut. Beide Eigenschaften resultieren aus der Ruckbestrafung in Quer- und Längsrichtung. In Tabelle 8.1 sind die objektiven Gütekriterien zusammengefasst. Es ist festzustellen, dass das PFA den größten minima-

Tabelle 8.1.: Ergebnisse für die vier Verfahren. Die besten Werte sind durch Fettdruck, die schlechtesten unterstrichen markiert.

\begin{tabular}{|c|c|c|c|c|}
\hline & \multicolumn{4}{|c|}{ Verfahren } \\
\hline Kriterium & PFA & OTO & MPPC & MPTSA \\
\hline $\min$. Abstand $[\mathrm{m}]$ & 1,86 & $\underline{1,41}$ & 1,48 & 1,60 \\
\hline max. Gesamtbeschl. $\left[\mathrm{m} / \mathrm{s}^{2}\right]$ & 9,87 & 9,15 & 8,47 & 10,01 \\
\hline max. Querbeschl. $\left[\mathrm{m} / \mathrm{s}^{2}\right]$ & $\underline{5,72}$ & 4,54 & 5,55 & 4,86 \\
\hline max. Längsbeschl. $\left[\mathrm{m} / \mathrm{s}^{2}\right]$ & $\underline{9,80}$ & 7,95 & 8,31 & 9,69 \\
\hline max. Schwimmwinkel $\left[^{\circ}\right]$ & $\underline{2,74}$ & 2,09 & 2,23 & 2,02 \\
\hline max. Gierrate [\%/s] & 14,87 & 13,03 & 14,43 & 11,38 \\
\hline
\end{tabular}

len Abstand der Schwerpunktstrajektorie zur hinteren linken Ecke des Hindernisses realisiert. Dementsprechend werden sowohl die höchsten Quer- und Längsbeschleunigungen als auch die höchsten fahrdynamischen Reaktionen aufgewendet. Lediglich die Gesamtbeschleunigung des MPTSA liegt höher. Das Ergebnis des OTO ist hierzu komplementär. Da der minimale Abstand zum Hindernis im Vergleich am geringsten ist, kommt das Verfahren mit einem geringeren Nutzungsgrad der Bewegungspotentiale als das PFA aus, wendet aber eine höhere Gesamtbeschleunigung als das MPPC und höhere fahrdynamische Zustandsgrößen als das MPTSA auf. Das MPPC erreicht einen größeren minmalen Abstand als das OTO und weist daher, mit Ausnahme der Gesamtbeschleunigung, höhere Nutzungsgrade der Bewegungspotentiale auf. Das MPTSA wendet im Vergleich mit dem MPPC niedrigere Maximalwerte der querdynamischen Größen, aber höhere Maximalwerte der längsdynamischen Größen auf und erreicht einen größeren minimalen Abstand. Aufgrund dieser Ergebnisse ist eine abschließende Bewertung noch nicht möglich, da jedes Verfahren Stärken und Schwächen aufweist. Darüber hinaus ist anzumerken, dass diese Ergebnisse von der Wahl der Gewichtungen und Auslegung der Regler abhängen. Die Nutzwertanalyse bietet im folgenden Abschnitt eine grundlegende Untersuchung und Entscheidungshilfe. 


\subsection{Vergleich mit der Nutzwertanalyse}

\section{Auswahl von Bewertungskriterien zur Nutzwertanalyse}

Bei der Auswahl von Kriterien zur Bewertung und dem darauf aufbauenden Vergleich müssen sich entgegenstehende Ziele Berücksichtigung finden. Bei dem vorliegenden Vergleich ist es zweckmäßig, zum einen Kriterien der Leistungsfähigkeit und zum anderen Kriterien des Aufwandes bzw. der Kosten zu berücksichtigen. Würde z.B. nur die Leistungsfähigkeit bewertet, so würde jeder Vergleich stets von der leistungsstärksten und damit häufig komplexesten und teuersten Lösung gewonnen, ohne dessen Chancen auf Realisierung zu betrachten. Ein solcher Vergleich wäre unvollständig. Analoge Betrachtungen ergeben sich, wenn nur Aufwands bzw. Kostenkriterien betrachtet werden.

Wie bei jeder Problemstellung ist die Komplexität der Lösung ein Kriterium. Unnötig komplexe Lösungen, welche keinen oder nur einen geringen Vorteil bieten, sind zu vermeiden. Die hier zu vergleichenden Verfahren sind zum Teil rechenintensiv und sollen auf einem Steuergerät im Fahrzeug implementiert werden. Aus Gründen der Kostenminimierung sind Steuergeräte weder besonders leistungsfähig noch ist eine Steigerung der Rechenleistung (und damit der Kosten) wünschenswert. Die Rechenlast ist daher ein wichtiges Kriterium. Fahrerassistenzsysteme und automatisierte Fahrfunktionen müssen immer wieder für verschiedene Fahrzeuge angepasst werden. Um die Kosten hierfür gering zu halten, darf der Applikationsaufwand nicht zu hoch ausfallen, woraus sich ein weiteres Kriterium ergibt. Um dem Fahrer in Notsituationen helfen zu können, muss das System eine bessere Regelungsgüte aufweisen als der Fahrer selbst und auch im Falle des autonomen Ausweichens sollte die Regelungsgüte möglichst hoch sein, um die Akzeptanz durch den Fahrer sicherzustellen. Bei einer zu geringen Regelungsgüte kann zudem auch bei kollisionsfreier Planung von Ausweichbahnen bzw. -trajektorien die Kollisionsfreiheit nicht gewährleistet werden. Die Regelungsgüte ist demnach ein weiteres wichtiges Kriterium. Bei einem assistierten Manöver ist es wichtig, die vom Fahrer induzierten Störungen auszuregeln. Das Kriterium der Störunterdrückung wird daher in die Untersuchungen aufgenommen. Ein Kollisionsvermeidungssystem greift nicht fortwährend in die Fahrzeugführung ein sondern nur in kritischen Situationen. Entsprechend müssen ein Kritikalitätsmaß und ein Auslösekriterium bzw. eine Eingriffsentscheidung festgelegt werden. Die verschiedenen Verfahren bieten unterschiedliche Möglichkeiten, die Kritikalität einer Situationen nach Definition A.1.14 zu approximieren, was in der Bewertung berücksichtigt wird. Es ist sinnvoll, die Adaptivität der Verfahren an verschiedene Verkehrssituationen zu bewerten, da sich hieraus mögliche Beschränkungen in der Anwendbarkeit ergeben. Die Adaptivitätseigenschaft ist in diesem Zusammenhang so zu verstehen, dass sich die Lösung (die optimale Trajektorie) an die Verkehrssituation anpasst (adaptiert). Im Gegensatz dazu wird das Verfahren selbst nicht an die jeweilige Verkehrssituation adaptiert. Durch die Entwicklung anderer Fahrerassistenzsysteme wie z.B. ABS, ASR, ESC, ACC oder AEB sind Algorithmen bekannt, welche online zumindest einige Fahrzeugparameter bestimmen. Hierzu gehören der Reibwert und die Gesamtmasse. Die Verfahren weisen unterschiedliche Möglichkeiten auf, diese Informationen zu nutzen. Demnach ergibt sich mit der Adaptivität an Parameteränderungen ein weiteres Kriteri- 
Tabelle 8.2.: Gruppierung von Bewertungskriterien.

\begin{tabular}{|c|c|c|}
\hline \multirow[t]{2}{*}{ Aufwandskriterien } & \multicolumn{2}{|c|}{$\begin{array}{l}\text { Leistungskriterien } \\
\end{array}$} \\
\hline & $\begin{array}{l}\text { grundsätzliche } \\
\text { Leistungskriterien }\end{array}$ & Adaptivitätskriterien \\
\hline Komplexität & Regelungsgüte & $\begin{array}{c}\text { Adaptivität an } \\
\text { verschiedene } \\
\text { Verkehrssituationen }\end{array}$ \\
\hline Rechenlast & Störunterdrückung & $\begin{array}{c}\text { Adaptivität an } \\
\text { Parameteränderungen }\end{array}$ \\
\hline Applikationsaufwand & Kritikalitätsmaß & \\
\hline
\end{tabular}

um. Die genannten Kriterien lassen sich, entsprechend den weiter oben ausgeführten Überlegungen, in jene gruppieren, die den Aufwand bewerten, und in solche, welche die Leistungsfähigkeit bewerten. Die letztgenannte Gruppe lässt sich weiter in grundsätzliche Leistungskriterien und Adaptivitätskriterien aufteilen. Die Zuordnung der einzelnen Kriterien ist Tabelle 8.2 zu entnehmen. Rechenzeiten werden nicht verglichen, da diese zum einen vom verwendeten Echtzeitsystem abhängen und zum anderen von der Programmiersprache bzw. von der Effizienz der Implementierung. Da für das MPPC Verfahren ein C++ Framework eingesetzt wird, aber alle anderen Verfahren in MATLAB/Simulink implementiert sind, ist ein Vergleich der Rechenzeit nicht sinnvoll. Zudem wird bei der Verwendung im Serieneinsatz die Hardware an die speziellen Anforderungen des Verfahrens angepasst werden. Weitere Bewertungskriterien liegen im Bereich der Robustheit, Sensitivität, Stabilität und Gewährleistung der Kollisionsfreiheit. Diese Kriterien sind jedoch nicht unabhängig voneinander. Beispielsweise wird der Nachweis der stabilen Fahrzeugführung nicht ausreichen, wenn nicht gleichzeitig die kollisionsfreie Fahrzeugführung bewiesen wird. Die Fragestellung müsste vielmehr lauten: „Für welches Verfahren kann am ehesten bzw. leichtesten die stabile, kollisionsfreie und robust leistungsfähige Fahrzeugführung garantiert werden?" Die Untersuchung dieser Fragestellung übersteigt den Rahmen dieser Arbeit und sollte Gegenstand weiterer Untersuchungen sein.

\section{Bewertender Vergleich mit der Nutzwertanalyse}

Im Folgenden werden die im vorherigen Abschnitt erläuterten Kriterien nacheinander betrachtet, um die Verfahren zu bewerten und zu vergleichen.

\section{Komplexität}

Die Komplexität des PFA ist im Vergleich gering. Die Bahnplanung besteht lediglich aus einem Polynom 5. Grades, dessen Bestimmung ähnlich ersichtlich ist wie die kaskadierten PD-Regler. Das Verhalten, insbesondere die Verteilung der Längs- und Querregelungsanteile, ist vorhersehbar. Verglichen mit dem Bahnfolgeverfahren liegt beim OTO eine höhere Komplexität vor. Die Trajektorienplanung, welche mehrere Ziele zur Laufzeit optimiert, weist eine höhere Komplexität auf. Das Verhalten ist durch 
die Wahl der Gewichte steuerbar und es ist a-priori ersichtlich, welches Verhalten durch eine Änderung bei der Wahl der Gewichte zu erwarten ist. Im Vergleich mit den anderen Verfahren weist das MPPC die höchste Komplexität auf. Das Gütemaß enthält die meisten Anteile und die Wahl der Gewichtungen ist aufwändig. Es ist daher häufig nicht ersichtlich, welchen Effekt die Änderung eines Gewichtes hat. Das MPTSA weist nur eine geringe Komplexität auf. Es müssen lediglich nichtlineare Zustandsdifferentialgleichungen gelöst werden, was mit einem geeigneten numerischen Integrationsverfahren gelingt. Das Verhalten ist vorhersehbar und die Wahl des Gütemaßes einfach.

\section{Rechenaufwand}

Die Berechnung des Polynoms als auch der Regler beim PFA erfordern nur wenig Rechenleistung. Der Rechenaufwand des OTO ist in diesem Vergleich mittelmäßig. Das MPPC Verfahren weist eine höhere, das Bahnfolgeverfahren eine geringere Rechenlast auf. Das MPTSA hat in etwa die gleiche Rechenlast, welche, im Gegensatz zu den Verfahren mit iterativer Online-Optimierung, deterministisch bestimmbar ist. Die genauen Rechenlasten sind abhängig von der konkreten Ausgestaltung des jeweiligen Verfahrens (z.B. Länge des Planungs- bzw. Prädiktionshorizontes) und werden hier daher nicht betrachtet.

\section{Applikationsaufwand}

Die Bahnplanung des PFA ist fahrzeugunabhängig und erfordert daher keine Anpassung. Die Reglerparameter bzw. Kennfelder erfordern die Identifikation von Fahrzeugparametern aus Fahrversuchen und die Applikation auf der Teststrecke. Zum Beispiel werden die stationären Vorsteuerungen des Gierratenreglers experimentell bestimmt. Aufgrund der Geschwindigkeitsabhängigkeit ist der Prozess mehrfach zu wiederholen. Dies gilt analog für die Fahrdynamikregler des OTO, bei welchem zusätzlich noch die Gewichte des Gütemaßes angepasst werden müssen. Um das Prädiktionsmodell des MPPC zu identifizieren, müssen Fahrversuche durchgeführt werden. Im Anschluss an die Identifikation müssen die Gewichte des Gütemaßes neu bestimmt werden. Wie beim MPPC muss das Prädiktionsmodell auch für das MPTSA mit Messungen aus dem Fahrversuch identifiziert werden. Da die Gewichte des Gütemaßes keine Funktionen der Modellparameter sind, ist eine Anpassung nicht nötig.

\section{Regelungsgüte}

Die erreichbare Regelungsgüte des PFA ist hoch, wie der Vergleich in der Standardsituation zeigt. Im linearen Bereich der Fahrdynamik ist die Regelungsgüte auch beim OTO hoch. Bei hochdynamischen Manövern sind größere Abweichungen zwischen Planung und Realisierung zu beobachten, was im Wesentlichen daraus resultiert, dass die Planung der Dynamik des geschlossenen Regelkreises nicht Rechnung trägt. Aufgrund des dynamischen Prädiktionsmodells und der optimalen Lösung des Optimalsteuerungsproblems beim MPPC ist die erreichbare Regelungsgüte im Sinne der Positioniergenauigkeit sehr hoch, wie es sich bei den in Kapitel 6 untersuchten 
Verkehrssituationen zeigt. Die Regelungsgüte des MPTSA ist aufgrund des modellprädiktiven Charakters und des genauen Prädiktionsmodells sehr gut. Die Suboptimalität macht sich hier nicht bemerkbar.

\section{Störunterdrückung}

Da die Ausweichbahn beim PFA nicht neu berechnet wird, sind die Möglichkeiten der Störunterdrückung im Vergleich zu den anderen Verfahren gering. Dennoch hat die Probandenstudie gezeigt, dass das Kollisionsvermeidungsverhalten des Fahrers verbessert werden kann. Die beiden modellprädiktiven Verfahren bieten den Vorteil, den Effekt einer Störung, falls diese bekannt ist, explizit während der Prädikiton zu berücksichtigen.

\section{Kritikalitätsmaß}

Die Approximation der Kritikalität beim PFA berücksichtigt lediglich die Querdynamik. Das Maß ist besser geeignet als die TTC, da die Verkehrssituation berücksichtigt wird. Im Vergleich mit den anderen Verfahren wird jedoch die Längsführung nicht berücksichtigt. Die modellprädiktiven Verfahren bieten darüber hinaus die Möglichkeit, neben den Beschleunigungen auch die fahrdynamischen Größen und die Stellgrößen zu berücksichtigen. Beim MPTSA besteht zusätzlich die Möglichkeit, die prädizierten aber nicht ausgewählten Trajektorien zu betrachten. Treten entlang dieser Trajektorien Kollisionen auf, kann dies auf eine kritische Situation hindeuten. Besondere Bedeutung hat die Trajektorie für die aktuell gewählten Stellgrößen und die Trajektorie des freien Systems. Der kurze Prädiktionshorizont schränkt die Approximation der Kritikalität ein.

\section{Adaptivität an Verkehrssituationen}

Das PFA ist ein starres Konzept, welches einem Hindernis ausweichen kann. Mehrere (bewegte) Hindernisse können nicht bzw. nur eingeschränkt berücksichtigt werden. Denkbar ist eine Erweiterung durch ein regelbasiertes Verfahren. Das OTO und MPPC adaptieren die Trajektorie sehr gut an unterschiedliche Verkehrssituationen, da das Gütemaß mehrere bewegte Hindernisse berücksichtigt. Das MPTSA berücksichtigt Hindernisse, deren prädizierte Aufenthaltsorte sich im Bereich des Trajektorienfächers befinden. Dadurch können weiter entfernte Hindernisse nicht berücksichtigt werden. Es stellt sich jedoch die Frage, wie diese detektiert werden können. Notmanöver werden bisher nur auf der Basis sehr sicherer Messungen ausgelöst. Der sichere Bereich von Radarsensoren beträgt derzeit ca. $30 \mathrm{~m}$. Bei höheren Geschwindigkeiten (der genaue Wert ist abhängig vom Prädiktionshorizont) überragt der Trajektorienfächer bereits den sicheren Messbereich. In Notsituationen liegen zudem häufig Hindernisse, wie z.B. Gegenverkehr, in der Sensorabdeckung. Aus diesen Überlegungen fällt der Nachteil des geringen Prädiktionshorizontes nicht stark ins Gewicht.

\section{Adaptivität an Parameteränderungen}

Alle verwendeten Regler des PFA und des OTO sind geschwindigkeitsabhängig mittels gesteuerter Adaption. Die Adaption an weitere Parameteränderungen ist durch 
die Erhöhung der Dimension der Kennfelder möglich. Allerdings steigt dann der Speicherbedarf und der Applikationsaufwand. Die Bahnplanung ist unabhängig von den Fahrzeugparametern und kann sich ändernde Parameter auf dem Planungshorizont nicht berücksichtigen. Eine solche Situation tritt beispielsweise auf, wenn die linke Fahrbahn mit Schnee bedeckt ist, während die rechte geräumt wurde und somit einen höheren Reibwert aufweist. Auch wenn die exakte Bestimmung des Reibwertes vor dem Fahrzeug bisher nicht möglich ist, können die Verfahren Schätzungen des Reibwertes unterschiedlich gut nutzen. Beim OTO können die Beschleunigungsgrenzen lokal gesenkt werden und die modellprädiktiven Verfahren können den Reibwert bei der Prädiktion berücksichtigen. Da das Gütemaß des MPTSA unabhängig von den Fahrzeugparametern ist, genügt es, das Prädiktionsmodell anzupassen. Beim MPPC kann es je nach Auswirkung der Parameteränderung auf die Dynamik der Regelstrecke notwendig sein, die Gewichtungen anzupassen.

\section{Scoring-Modell}

Mit der Nutzwertanalyse bzw. dem Scoring-Modell werden die Vor- und Nachteile der Verfahren bewertet, indem die fünf Bewertungsstufen

$$
\begin{array}{rll}
-- & \hat{=} & \text { "sehr schlecht" } \\
- & \hat{=} & \text { "schlecht" } \\
0 & \hat{=} & \text { "durchschnittlich" } \\
+ & \hat{=} & \text { "gut" } \\
++ & \hat{=} & \text { "sehr gut" }
\end{array}
$$

verwendet werden. In der Tabelle 8.3 sind die Bewertungen für die einzelnen Verfahren aufgelistet.

Tabelle 8.3.: Bewertung der Verfahren bezüglich der zuvor erläuterten Kriterien.

\begin{tabular}{|l|l|c|c|c|c|}
\hline \multicolumn{2}{|c|}{ Kriterien } & \multicolumn{4}{c|}{ Verfahren } \\
\hline Kriteriengruppe & Einzelkriterium & PFA & OTO & MPPC & MPTSA \\
\hline \hline \multirow{3}{*}{ Aufwandskriterien } & Komplexität & + & - & -- & ++ \\
& Rechenaufwand & ++ & 0 & -- & 0 \\
& Applikationsaufwand & - & 0 & + & ++ \\
\hline \multirow{3}{*}{ grunds. Leistungs. } & Regelungsgüte & 0 & 0 & ++ & ++ \\
& Störunterdrückung & - & + & ++ & ++ \\
& Kritikalitätsmaß & 0 & + & ++ & + \\
\hline \multirow{2}{*}{ Adaptivitätskrit. } & Adaptivität Verkehrs. & -- & ++ & ++ & + \\
& Adaptivität Parameter & - & - & + & ++ \\
\hline
\end{tabular}

Die Bewertungen unterliegen zwar der Erfahrung des Autors, sie ergeben sich jedoch aus den in dieser Arbeit aufgezeigten Eigenschaften. Bei der Nutzwertanalyse werden den einzelnen Kriterien Gewichtungen und den Bewertungen numerische Werte zugewiesen, sodass sich ein Gesamtwert (Score) für jedes Verfahren ergibt und damit eine Rangfolge ableitbar ist. Die Wichtigkeit (und damit die Gewichtung) eines Kriteriums 
ist jedoch abhängig vom gewählten Zielsystem (z.B. assistierend oder autonom eingreifende Funktion, Sensorkonfiguration) und subjektiven Einflüssen. Zum Beispiel wird für eine Applikation für ein Fahrzeug der Luxusklasse der Rechenaufwand und damit der Preis für das entsprechende Steuergerät weniger wichtig sein als für eine Applikation in einem Kleinwagen. In Abschnitt 8.5 werden von den Gewichtungen der Kriterien unabhängige Aussagen zur Auswahlentscheidung eines Verfahrens vorgestellt. Zunächst werden aber mögliche Modifikationen und Kombinationen der Verfahren erläutert.

\subsection{Modifikationen und Kombinationen der Verfahren}

Die bisherigen Betrachtungen haben gezeigt, dass jedes Verfahren Vor- und Nachteile aufweist, wobei der Nachteil des einen oft der Vorteil des anderen Verfahrens ist. Die Idee ist es, durch die Kombination von zwei Verfahren die Nachteile des einen durch die Vorteile des anderen auszugleichen. Hier bietet sich das OTO in Kombination mit dem MPTSA an, wobei das MPTSA als reiner Regler fungiert und die Sollgrößen vom OTO übernimmt (MPTSCE) oder das MPTSA zusätzlich zur Trajektorienfolgeregelung zur lokalen Kollisionsvermeidung eingesetzt wird. Zwischen diesen beiden Varianten kann durch die Wahl der Gewichtungen $\gamma_{X}$ und $\gamma_{Y}$ des Gütemaßes JMPTSA stufenlos gewählt werden.

$$
J_{\text {MPTSA }}=\sum_{k=1}^{n_{\mathrm{p}}}\left(\gamma_{\Psi} \Psi_{k}^{2}+\gamma_{\mathrm{env}} \Gamma_{\mathrm{pf}, \mathrm{k}}+\gamma_{\mathrm{s}} s_{\mathrm{soll}, k}+\gamma_{\mathrm{Y}}\left(Y_{\mathrm{soll}}-Y\right)^{2}+\gamma_{\mathrm{X}}\left(X_{\mathrm{soll}}-X\right)^{2}\right)
$$

Durch die Kombination der Verfahren wird der kurze Prädiktionshorizont des MPTSA durch das OTO ausgeglichen. Dennoch bleiben die Vorteile eines modellprädiktiven Verfahrens erhalten.

Der wesentliche Nachteil des MPPC ist die hohe Rechenlast. Das verwendete Prädiktionsmodell weist eine hohe Ordnung $(n=9)$ auf, was für MPC Anwendungen bei mechatronischen Systemen ungewöhnlich ist. Die Modellordnung kann aber nicht weiter reduziert werden, ohne die Modellgüte ebenfalls stark zu reduzieren oder den physikalischen Bezug des Modells zu verlieren. Alternativ soll ein unterlagerter Fahrdynamikregler mit geringem Rechenaufwand eingesetzt werden. Im oberen Teil von Abbildung 8.2 ist das entsprechende Blockschaltbild dargestellt. Anders als bei modellprädiktiven Verfahren kann der Fahrdynamikregler die Sollschlüpfe an den vier Rädern unterschiedlich wählen. Bei einem modellprädiktiven Regler würde dies zu einer extrem hohen Rechenzeit und Komplexität führen. Eine Anforderung an den Fahrdynamikregler ist, dass sich der geschlossene Regelkreis mit wenig Aufwand modellieren lässt. Wenn jeweils ein Modell erster Ordnung für das Übertragungsverhalten der drei fahrdynamischen Größen $\beta, \dot{\Psi}$ und $v$ ausreicht, wäre es gelungen, die Ordnung des Prädiktionsmodells von $n=9$ auf $n=6$ zu reduzieren (im Folgenden mit $\mathrm{MPPC}_{6}$ abgekürzt). Die Vorgehensweise weist aber auch Nachteile auf. So geht die inhärente Adaptivität an Parameteränderungen verloren. Vielmehr müsste das Verhalten des Fahrdynamikreglers bei unterschiedlichen Parametersätzen modelliert werden. Zudem ist die Regelungsgüte des $\mathrm{MPPC}_{6}$ an die Regelungsgüte des Fahrdynamikreglers gebunden. Die Vorgehensweise lässt sich wiederholen und ein Positions- 


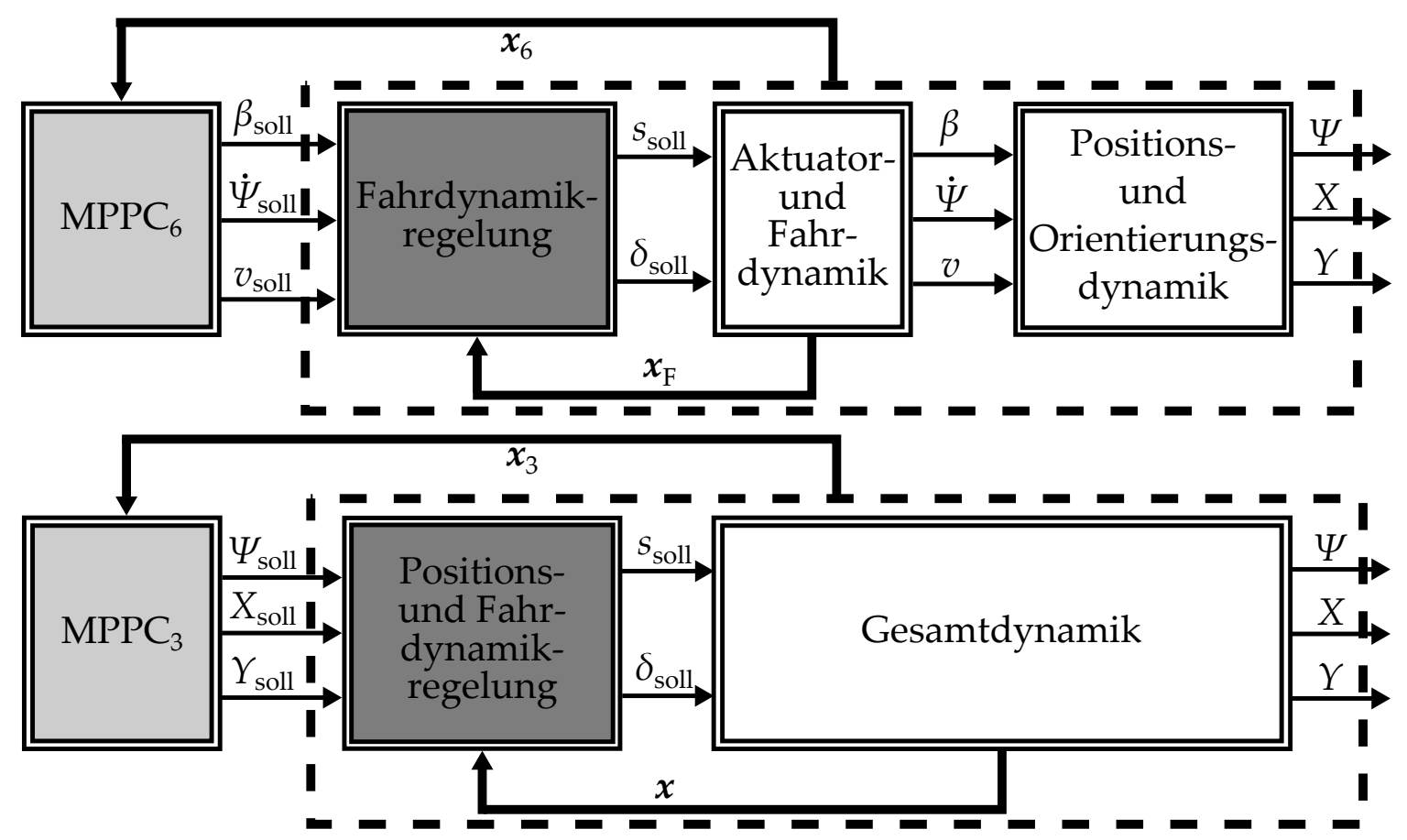

Abbildung 8.2.: Blockschaltbilder der Möglichkeiten das MPPC mit einer unterlagerten Regelung zu kombinieren.

und Orientierungsregler kann ergänzt werden. Das zugehörige Blockschaltbild ist in Abbildung 8.2 unten zu erkennen. Gelingt es mit dem Orientierungs- und Positionsregler eine mit einem System erster Ordnung zu modellierende Dynamik einzustellen, so lässt sich die Modellordnung auf $n=3$ reduzieren (im Folgenden $\mathrm{MPPC}_{3}$ genannt). Die zuvor genannten Nachteile bleiben aber erhalten. Hinzu kommt, dass die Stabilität der Fahrdynamik nicht mehr durch das modellprädiktive Verfahren beeinflusst wird. Damit ist es nicht mehr möglich, das Fahrzeug in bestimmten Situationen zu destabilisieren, um durch die erhöhte Gierdynamik ein höheres Kollisionsvermeidungspotential zu erreichen.

Das MPTSA bzw. MPTSC hat sich als leistungsfähiger Positions-, Orientierungs- und Fahrdynamikregler erwiesen. Steht genug Rechenleistung zur Verfügung, so kann der unterlagerte Regler durch das MPTSA bzw. MPTSC realisiert werden. Diese Vorgehensweise ähnelt der Kombination von OTO und MPTSC bzw. MPTSA. Der Unterschied besteht darin, dass beim $\mathrm{MPPC}_{3}$ die Dynamik des geschlossenen unterlagerten Regelkreises berücksichtigt wird, während dies beim OTO nicht möglich ist.

Mit dem MPTSA eröffnet sich eine weitere Möglichkeit, eine universelle Assistenzfunktion zur Kollisionsvermeidung zu entwickeln. Hierfür muss jedoch die Sichtweise auf die Funktion geändert werden. Bisher wurde versucht, die optimale Trajektorie zu einer Verkehrssituation zu finden und diese dem Fahrer ab der Eingriffsentscheidung durch eine kontinuierliche Regelung zu vermitteln. Die Vorgehensweise kann auch für die autonome Fahrzeugführung in Notsituationen angewendet werden. Die Absicherung eines derartigen Systems stellt jedoch eine große Herausforderung dar. Ändert man hingegen die Sichtweise und beschränkt sich in der Tradition der klassischen aktiven Sicherheitssysteme auf das Erkennen und Korrigieren von Fahrfehlern durch diskrete 
Eingriffsimpulse, vereinfacht sich die Aufgabenstellung. Denn statt die optimale Trajektorie bestimmen zu müssen, genügt es festzustellen, dass die vom Fahrer gewählte Trajektorie bzw. Stellgröße ungeeignet ist bzw. zu einer Kollision führen wird. Durch die Trajektorienschar des MPTSA werden Informationen über kollisionsbehaftete und kollisionsfreie Trajektorien- bzw. Stellgrößenbereiche zur Verfügung gestellt, auf deren Basis eine qualifizierte Eingriffsentscheidung und -richtung getroffen werden kann. Die Funktion ist nicht nur auf Notsituationen beschränkt. Wenn der Fahrer in einer engen Rechtskurve einen zu großen Lenkradwinkel wählt und droht den Bordstein zu touchieren, kann mit der Trajektorienschar festgestellt werden, dass der vom Fahrer gewählte Lenkradwinkel und auch alle größeren Lenkradwinkel in einer Kollision enden. Gleichzeitig werden im Bereich kleinerer Lenkradwinkel kollisionsfreie Trajektorien gefunden. Ohne die optimale Trajektorie zu bestimmen, kann gefolgert werden, dass der Lenkradwinkel verringert werden muss. Entsprechend kann ein Lenkmomentenimpuls nach links überlagert werden. Die Vorgehensweise hat den weiteren Vorteil, dass die Impulsform des Überlagerungsmomentes frei gestaltbar ist und sich nicht indirekt als Stellgröße eines Regelkreises ergibt. Die speziellen Anforderungen an Mensch-Maschine-Schnittstellen können somit berücksichtigt werden.

\subsection{Fazit}

Wenn die Kriterien der Nutzwertanalyse entsprechend ihrer Gruppierung zusammengefasst werden, können die Verfahren in einem Diagramm nach Abbildung 8.3 eingetragen werden. Auf der Abszisse werden die Leistungskriterien zusammengefasst

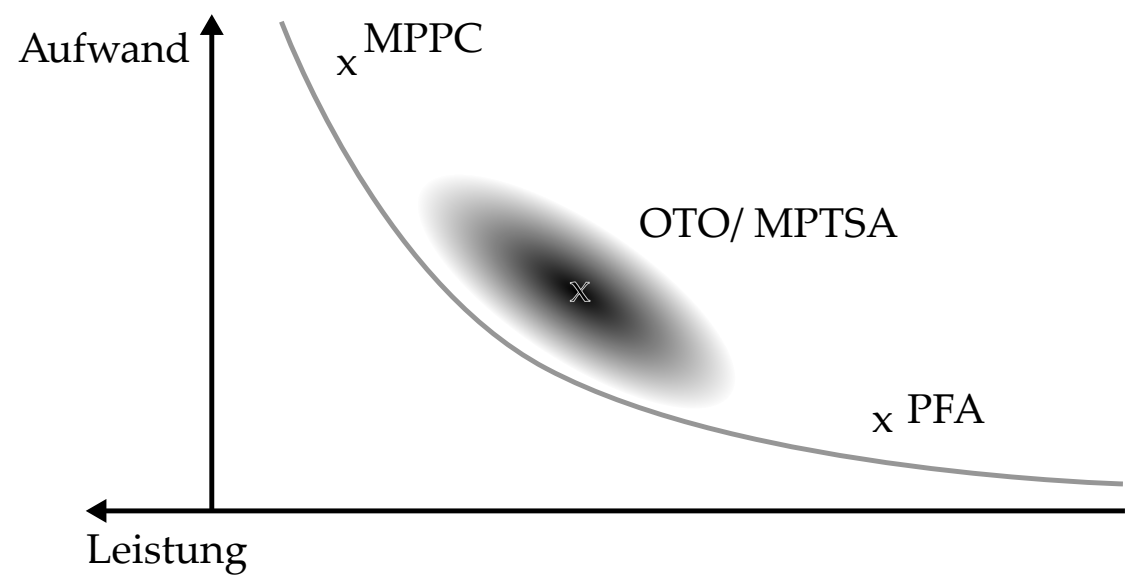

Abbildung 8.3.: Approximation der Paretofront (graue Linie) durch die vier Verfahren.

und auf der Ordinate die Kosten bzw. Aufwandskriterien. Es zeigt sich, dass diese Kriteriengruppen einen Zielkonflikt hervorrufen, der durch die vier Verfahren mit unterschiedlichen Kompromissen gelöst wird. Die Einordnung der Verfahren approximiert die Paretofront. Das MPPC weist sowohl die höchste Leistungsfähigkeit als auch den höchsten Aufwand auf. Das PFA stellt in diesem Vergleich die komplementäre Kompromisslösung dar. Die Einordnung des MPTSA und des OTO ist nicht eindeutig und davon abhängig, wie die begrenzte Adaptivität an Verkehrssituationen des MPTSA im Vergleich zum OTO und MPPC gewichtet wird. Unabhängig von der 
Gewichtung der einzelnen Kriterien, lassen sich die folgenden Aussagen über die Anwendungsmöglichkeiten der einzelnen Verfahren treffen:

- Das PFA ist eine geeignete Alternative, wenn ein Assistenzsystem zum Notausweichen benötigt wird, eine nach vorne gerichtete Sensorkonfiguration verwendet wird und die Rechenleistung des Steuergerätes für das MPTSA nicht ausreichend ist. Die Eingriffe des Systems müssen dabei vom Fahrer zu übersteuern sein, sodass der Fahrer in der Verantwortung bleibt. Sowohl die Initiierung des Notmanövers durch eine deutliche Lenkbewegung als auch die Beobachtung des Verkehrs müssen vom Fahrzeugführer durchgeführt werden. Durch die Kombination von ACC und Lane Guidance entsteht bereits eine einfache Form des automatisierten Fahrens. Die Funktion beschränkt sich jedoch auf die Nutzung einer Fahrspur. Durch die Integration einer Bahnplanung ähnlich der des PFA, d.h. eine polynomiale Spurwechselbahn, ließe sich die Funktion auf mehrere Fahrstreifen erweitern. Wenn der Fahrer den Spurwechselwunsch z.B. durch Betätigen des Blinkers anzeigen muss, genügt auch hier eine nach vorne gerichtete Sensorkonfiguration. Da beim PFA nur bedingt mehrere bewegte Hindernisse berücksichtigt werden können, eignet sich das Verfahren weder für autonome Notausweichmanöver noch für fortschrittliche automatisierte Fahrfunktionen.

- Unter der Annahme, dass die Umfeldsensoren nur derart begrenzte Informationen bereitstellen wie sie vom MPTSA berücksichtigt werden können, ergeben sich praktisch keine Nachteile für die Kollisionsvermeidung des MPTSA gegenüber dem OTO oder MPPC. Für automatisierte Fahrfunktionen, welche zu einem gewissen Grad realisiert werden können, gilt diese Aussage nicht. Es gibt Aufgaben, die nur durch zusätzliche Algorithmen (zum Beispiel globale Bahnplanung) gelöst werden können. Hierzu gehört z.B. das mehrzügige Einparken oder das Einfädeln auf Autobahnen, was unter Umständen einen längeren Prädiktionshorizont benötigt.

- Liegen zukünftig umfassendere Informationen über die Bewegung der Verkehrsteilnehmer vor und wird im Zuge des automatisierten Fahrens die autonome Kollisionsvermeidung zwingend erforderlich, ist das MPPC oder eine Kombination aus OTO und MPTSA anzuwenden. Das OTO und das MPPC eignen sich beide sowohl zur assistierten und autonomen Kollisionsvermeidung als auch zum automatisierten Fahren nach Anpassung der Gütefunktion. Die OnlineOptimierung beider Verfahren erlaubt es, komplexe Verkehrssituationen und Aufgaben zu lösen und es besteht die Möglichkeit, durch die Berechnung mehrerer Alternativtrajektorien die global optimale Trajektorie zu finden. Das OTO weist jedoch die in dieser Arbeit aufgezeigten Nachteile bei der Fahrzeugführung in Notsituationen auf, sodass die Ergänzung mit dem MPTSA zielführend ist. 


\section{9}

\section{Zusammenfassung und Ausblick}

Die Unfallzahlen zeigen, dass viele Unfälle durch Fehlverhalten des Fahrers verursacht werden. Diesem Umstand Rechnung tragend wurden Fahrerassistenzsysteme entwickelt, welche den Fahrer unterstützen bzw. entlasten. In der Folge konnten die Unfallzahlen gesenkt werden. Jedoch ist bei den seit 2013 wieder vermehrt auftretenden Unfällen weiterhin das Fehlverhalten der Fahrer eine der häufigsten Unfallursachen. Es ist daher sinnvoll, weitere Assistenzsysteme bzw. automatisierte Fahrfunktionen zu entwickeln, welche vor allem Informationen über die Fahrzeugumgebung berücksichtigen. Viele Fahrer sind vor allem in kritischen Situationen nicht in der Lage, ihr Fahrzeug adäquat zu führen, sodass durch die Entwicklung des Notausweichassistenten davon auszugehen ist, dass in solchen Situationen die Kollisionswahrscheinlichkeit gesenkt wird. Zur Entwicklung derartiger Systeme wird ein dreistufiger Prozess bestehend aus Simulation, Fahrsimulator und Versuchsfahrzeug vorgeschlagen, um den Aufwand durch Erprobung und Probandenstudien zu reduzieren. Aufgrund der Reproduzierbarkeit und der Möglichkeit, Probanden überraschend mit kritischen Situationen zu konfrontieren, wird ein feststehender Fahrsimulator eingesetzt, um in einer Probandenstudie die Wirksamkeit der Lenkmomentenüberlagerung zur Reduktion der Kollisionswahrscheinlichkeit aufzuzeigen.

Dadurch motiviert werden vier unterschiedliche Verfahren vorgestellt, mit denen eine solche Assistenz realisiert werden kann. Das Bahnfolgeverfahren ist ein einfaches aber nicht vielfältiges Verfahren, welches mit wenig Rechenleistung auskommt aber nur eine begrenzte Anzahl an Verkehrssituationen lösen kann. Aufgrund der geringen Leistungsfähigkeit des Normalfahrers lässt sich aber mit diesem Verfahren bereits eine assistierend eingreifende Funktion realisieren. Hierzu wird zunächst eine kollisionsfreie Bahn, welche durch ein Polynom 5. Grades beschrieben wird, um das Hindernis herum berechnet und anschließend mit einem auf PD-Reglern basierenden kaskadierten Querregelungskonzept entwickelt. Die Querführung wird durch eine fahrergesteuerte Längsregelstrategie ergänzt, um kombinierte Bremsausweichmanöver zu ermöglichen. Um möglichst viele Verkehrssituationen lösen zu können, wird das rechenaufwändigere Online-Trajektorienoptimierungsverfahren entwickelt, welches ursprünglich für Anwendungen in der mobilen Robotik eingesetzt wurde. In jedem Abtastschritt wird basierend auf kinematischen Größen eine Trajektorie optimiert, wobei durch das Gütemaß mehrere sich entgegengesetzte Ziele Berücksichtigung finden. Die ermittelte Trajektorie ist kollisionsfrei und minimiert fahrdynamische Reaktionen während eines Ausweichmanövers. Durch die fortlaufende Neuberechnung wird eine 
Positionsregelung inhärent realisiert, welche durch unterlagerte Fahrdynamikregelungen ergänzt wird. Die Planung weist jedoch aufgrund des zugrundeliegenden Massenpunktmodells eine beschränkte Präzision auf, weshalb beim modellprädiktiven Planungs- und Regelungsverfahren ein dynamisches Modell der Fahrzeugbewegung integriert wird. Durch die Formulierung als Optimalsteuerungsproblem ergeben sich als Lösung neben der optimalen Trajektorie auch die optimalen Stellgrößen, welche direkt auf die Regelstrecke angewendet werden können. Aufgrund der hohen Genauigkeit des Prädiktionsmodells lässt sich eine hohe Positioniergenauigkeit erreichen. Im Vergleich zum Online-Trajektorienoptimierungsverfahren steigt die Rechenlast aber nochmals an. Das modellprädiktive Trajektorienscharverfahren reduziert den Rechenaufwand durch eine suboptimale Lösung des Optimalsteuerungsproblems mit dem Ziel, die Vorteile eines modellprädiktiven Verfahrens zu erhalten. Hierzu werden die Stellgrößen grob diskretisiert und auf dem Prädiktionshorizont konstant gehalten. Auf diese Weise können bereits viele Verkehrssituationen gelöst werden. Der kurze Prädiktionshorizont verhindert jedoch, dass alle Hindernisse in die Planung mit einbezogen werden und eine Erweiterung auf das globale Optimum wie beim OTO und MPPC nicht möglich ist. Aufgrund der begrenzten Umfelderfassung ist die praktische Relevanz dieses Nachteils für eine assistierende Funktion vernachlässigbar.

Der Vergleich der Verfahren mit der Nutzwertanalyse zeigt, dass die vier Verfahren den Zielkonflikt zwischen Aufwand und Leistungsfähigkeit mit unterschiedlichen Kompromissen lösen. Die Wahl eines der Verfahren ist abhängig von den konkreten Systemanforderungen (Assistenzsystem oder autonome Funktion, Kollisionsvermeidung oder automatisierte Fahrfunktion). Für assistierende Funktionen eignet sich das PFA und das MPTSA unter der Bedingung, dass der Fahrer in der Verantwortung bleibt. Das MPTSA kann darüber hinaus zur autonomen Kollisionsvermeidung eingesetzt werden, solange nur entsprechend begrenzte Umfeldinformationen zur Verfügung stehen, da unter dieser Bedingung keine Nachteile gegenüber dem OTO oder MPPC entstehen. Liegen umfassendere Umfeldinformationen vor, eignet sich das OTO, eine Kombination von OTO und MPTSA und das MPPC. Die Kombination von OTO und MPTSA ist eine vielversprechende Alternative, da der Rechenaufwand und die Komplexität des MPPC vermieden werden, aber zum einen die Fähigkeiten zur globalen Trajektorienplanung durch das OTO und zum anderen die modellprädiktiven Eigenschaften des MPTSA eingesetzt werden können.

Als Ausblick auf weitere Forschungsthemen ist die Generalisierung der Trajektorienplanung durch das OTO oder den MPPC auf das globale Optimum zu nennen. Hier können die Erkenntnisse aus der mobilen Robotik erneut einfließen (siehe [Rös+15a]). Ein weiteres Themengebiet besteht in der Prädiktion der Bewegung der Verkehrsteilnehmer und der Berücksichtigung der Unsicherheit über die Aufenthaltsorte bei der Planung. Gierstabilisierungssysteme werden in jedem Neufahrzeug eingesetzt. Hier besteht die Herausforderung darin, die Gesamtdynamik aus Regelung und Fahrdynamik für die Prädiktion zu modellieren. Abschließend sollten unterschiedliche Umwelteinflüsse wie Reibwertänderungen untersucht werden. Im Anhang A wird in einem ersten beispielhaften Vergleich die Generalisierbarkeit der modellprädiktiven Trajektorienscharregelung aufgezeigt. Weitere Forschungsprojekte sollten diese Frage umfassender untersuchen und die Ähnlichkeit zu den bekannten Verfahren der modellprädiktiven Regelung aufzeigen. 


\section{A}

Anhang

Der Anhang gliedert sich in die beiden Teile Definitionen und modellprädiktive Trajektorienscharregelung (MPTSC).

\section{A.1. Definitionen}

Im Folgenden werden einige Begriffe des Straßenbaus und der Fahrzeugführung, welche in der Literatur nicht immer einheitlich verwendet werden und es daher $\mathrm{zu}$ Missverständnissen kommen kann, definiert und erläutert. Anschließend werden Definitionen zur Kritikalität von Verkehrssituationen vorgestellt und mit Beispielen erläutert. Es folgen Definitionen von Gütekriterien zur Bewertung von Signalverläufen.

\section{A.1.1. Begriffe des Straßenbaus}

\section{Definition A.1.1: Fahrbahn}

Die Fahrbahn ist die im Normalfall befahrbare Fläche der Straße. Sie besteht aus den Fahr- und Standstreifen.

\section{Definition A.1.2: Fahrspur/Fahrstreifen}

Die Fahrspur bzw. der Fahrstreifen sind durch entsprechende Markierungen auf der Fahrbahn gekennzeichnete Bereiche, in denen das Fahrzeug in der Regel dauerhaft zu führen ist. Fahrstreifenwechsel sind bei gestrichelten Markierungslinien erlaubt.

\section{Definition A.1.3: Verkehrsfläche}

Die Verkehrsfläche besteht aus der Fahrbahn und weiteren Flächen, welche nicht zum Befahren mit Kraftfahrzeugen gedacht sind, aber von anderen Verkehrsteilnehmern wie Fußgängern und Radfahrern genutzt werden dürfen.

\section{Definition A.1.4: Kurve (umgangssprachlich)}

Umgangssprachlich ist mit einer Kurve ein gekrümmter Fahrbahnverlauf gemeint.

Aufgrund der weiten Verbreitung dieser Bezeichnung wird diese auch in der vorliegenden Arbeit verwendet, obwohl der mathematische Begriff der Kurve eine andere Bedeutung hat (siehe Definition A.1.10). 


\section{A.1.2. Begriffe der Fahrzeugführung}

\section{Definition A.1.5: Fahrkorridor}

Der Fahrkorridor ist der Teil der Fahrbahn, welcher durch das Ego-Fahrzeug befahrbar ist und nicht durch Hindernisse besetzt ist.

\section{Definition A.1.6: Fahrschlauch}

Der Fahrschlauch ist die zweidimensionale Projektion des Fahrzeugaufbaus, welche das Fahrzeug während der Fahrt überstreicht.

\section{Definition A.1.7: Trajektorie}

Eine Trajektorie ist die Beschreibung der Bewegung eines Punktes $(X(t), Y(t))$ des Fahrzeugs (meist Schwerpunkt) in Abhängigkeit der Zeit. Alle kinematischen Größen sind eindeutig bestimmt.

\section{Definition A.1.8: Bahn}

Eine Bahn ist die Beschreibung der Bewegung eines Punktes $Y(X)$ des Fahrzeugs (meist Schwerpunkt) ohne Zeitinformationen. Aufgrund der fehlenden Zeitinformation sind die kinematischen Größen nicht vollständig bestimmt.

\section{Definition A.1.9: Pfad}

Der Pfad ist ein Synonym für Bahn.

Diese Definition wird aufgrund der Redundanz zu dem Begriff Bahn in der vorliegenden Arbeit nicht verwendet.

Definition A.1.10: Kurve (mathematisch)

Im mathematischen Sinne ist eine Kurve der Graph einer Funktion.

Diese Definition wird aufgrund der Verwechslungsgefahr mit dem umgangssprachlichen Begriff (siehe Definition A.1.4) in der vorliegenden Arbeit nicht verwendet.

\section{Definition A.1.11: Bahnkurve}

Die Bahnkurve ist ein Synonym für Trajektorie.

Diese Definition wird aufgrund der missverständlichen Bezeichnung und der Verwechslungsgefahr mit den Begriffen Bahn und/oder Kurve nicht verwendet. 


\section{A.1.3. Zur Kritikalität von Verkehrssituationen}

\section{Definition A.1.12: Bewegungspotentiale}

Das Bewegungspotential eines Fahrzeugs und dessen Aktoren entspricht den maximal möglichen Werten der dynamischen Größen $Q_{d y n, \max , i}(i=1,2, \ldots$, Anzahl der Größen), welche durch das Fahrzeug und/oder dessen Aktoren erreicht werden können. Grenzwerte können zusätzlich eingeschränkt werden, um die Stabilitätsgrenze zu berücksichtigen. Ein weiterer Grund zur Reduktion eines Grenzwertes kann die Fähigkeit des Fahrers sein. Die Definition mehrerer Bewegungspotentiale ist dann erforderlich, wenn in unterschiedlichen Verkehrssituationen unterschiedliche Bewegungspotentiale zuerst erreicht werden.

Beispiel A: Die maximal mögliche Gesamtbeschleunigung ist durch den Kammschen Kreis beschränkt. Das Beschleunigungspotential liegt auf trockener Straße bei $a \approx \mathrm{g}$. Analog lassen sich auch für andere dynamische Größen (Gierrate, Lenkrate, ...) obere Grenzwerte (Gierratenpotential, Lenkratenpotential, ...) bestimmen.

Beispiel B: Der Schwimmwinkel kann Werte zwischen $\beta= \pm 180^{\circ}$ annehmen. Aus Gründen der Stabilität ist es sinnvoller, das Schwimmwinkelpotential auf einen kleineren Wert (z.B. $\beta= \pm 5^{\circ}$ ) zu definieren. In [Thi15] wird die Phasenebene des Einspurmodells betrachtet und die Kritikalität anhand des Abstandes zur Stabilitätsgrenze bewertet. Das heißt, dass das Schwimmwinkelpotential und das Gierratenpotential sind nicht konstant und insbesondere abhängig vom fahrdynamischen Zustand.

Beispiel C: Die maximal erreichbare Lenkrate des Lenkaktuators kann höher liegen als die Werte, bei denen dem Fahrer typischerweise die Fahrzeugführung zuzutrauen ist. Das Lenkratenpotential kann daher kleiner gewählt werden.

Definition A.1.13: Ideale Kollisionsvermeidungstrajektorie:

Die ideale Kollisionsvermeidungstrajektorie (KVT) ist eine fiktive Trajektorie, welche in jeder Situation von allen Betrachtern als die bestmögliche Lösung einer kritischen Verkehrssituation $z u$ bezeichnen ist. Die ideale KVT enthält die dynamischen Größen $Q_{d y n, i, k}(k=1,2, \ldots, A n z a h l$ der Stützstellen der Trajektorie), welche die Fahrzeugbewegung beschreiben.

Erläuterung: Die ideale KVT unterscheidet sich von der optimalen KVT dadurch, dass ihre Optimalität nicht mit einem beliebigen objektiven Gütemaß bestimmt ist, sondern vielmehr das Minimum des fiktiven idealen Gütemaßes ist. Bei der Lösung der Fahrzeugführungsaufgabe sind vielfältige und komplexe Aspekte zu berücksichtigen. Zum Beispiel sind Kollisionen zu vermeiden und eine komfortable Fahrweise erstrebenswert. Der Entwickler eines Fahrzeugführungssystems wird versuchen, alle Aspekte in einem Gütemaß zu berücksichtigen, was ihm im Allgemeinen nicht gelingen wird, da die Gesamtheit aller Aspekte unbekannt ist. Jedoch existiert theoretisch ein ideales Gütemaß, welches eben alle Aspekte der Fahrzeugführungsaufgabe derart quantitativ berücksichtigt, dass alle Betrachter die resultierende Trajektorie als die bestmögliche bezeichnen.

Definition A.1.14: Kritikalität als Nutzungsgrad der Bewegungspotentiale Es gilt für die Kritikalitätsindikatoren:

$$
K I B_{i}=\max _{k}\left(\frac{\left|Q_{d y n, i, k}\right|}{Q_{d y n, \max , i, k}}\right) .
$$


Die Kritikalität basierend auf dem Nutzungsgrad der Bewegungspotentiale entspricht dem Maximum der Kritikalitätsindikatoren:

$$
K_{p o t}=\max _{i}(K I B)
$$

Definition A.1.15: Kritikalität aufgrund der Unsicherheit über die Bewegung der Verkehrsteilnehmer

Der Kritikalitätsindikator $K I U_{j}$ ist gegeben durch die Inverse des minimalen Abstandes $d_{m i n}, j, k$ ( $j=1,2, \ldots$, Anzahl der Hindernisse) zwischen dem Ego-Fahrzeug und einem Hindernis bezogen auf einen Mindestabstand $d_{\text {soll }, j, k}$ und ist wie folgt definiert:

$$
\operatorname{KIU}_{j}=\max _{k}\left(\left\{\begin{array}{ll}
\frac{d_{\text {soll }, j, k}}{d_{\min , j, k}} & \text { wenn } d_{\text {min }, j, k}>d_{\text {soll }, j, k} \\
1 & \text { sonst }
\end{array}\right)\right.
$$

Daraus ergibt sich die Kritikaltität aufgrund der Unsicherheit über die Bewegung der Verkehrsteilnehmer:

$$
K_{u n s}=\max _{j}(K I U)
$$

Anmerkung: Der Mindestabstand $d_{\text {soll }, j, k}$ ist im Allgemeinen nicht konstant.

\section{Definition A.1.16: Definition der Kritikalität einer Verkehrssituation:}

Die Kritikalität einer Verkehrssituation teilt sich auf in die Kritikalität, welche aus dem Nutzungsgrad der Bewegungspotentiale entsteht, und in die Kritikalität, welche aus den Abständen des Ego-Fahrzeugs zu Hindernissen entsteht. Die Gesamtkritikalität $K_{g e s}$ entspricht dem Maximum der beiden Kritikalitäten:

$$
K_{g e s}=\max \left(K_{p o t}, K_{u n s}\right) .
$$

Anmerkung: Die Kritikalität ist eine dimensionslose Größe mit einem Wertebereich von $0 \%$ bis $100 \%$.

Im Folgenden soll anhand von zwei Beispielsituationen gezeigt werden, warum zwei unterschiedliche Kritikalitätsmaße benötigt werden:

Das Ego-Fahrzeug fährt auf einer Straße mit zwei Spuren für diese Fahrtrichtung. Es nähert sich einem stehenden Hindernis. In dieser Situation kann mit dem Kritikalitätsmaß $K_{\text {uns }}$ nur bedingt eine Aussage zur Kritikalität der Situation gemacht werden, da z.B. die Größe des Hindernisses einen Einfluss auf die Kritikalität aber nicht auf das Maß $K_{\text {uns }}$ hat. Dies gilt analog für die Straßenbreite. Das Kritikalitätsmaß $K_{\text {pot }}$ berücksichtigt die vorgenannten Faktoren und ist hier daher besser geeignet.

Als zweites Beispiel wird eine Kreuzungssituation betrachtet, in der ein Fahrzeug in Nord-Süd-Richtung fährt und das andere in West-Ost-Richtung. Beide bewegen sich geradlinig gleichförmig und passieren in sehr kurzem Abstand die Kreuzung. Aufgrund der Art der Bewegung ist der Nutzungsgrad aller Bewegungspotentiale identisch Null und das Kritikalitätsmaß $K_{\text {pot }}$ eignet sich nicht ausschließlich, um die Kritikalität der Situation zu beschreiben. Wenn die Bewegungen beider Fahrzeuge exakt bekannt sind, ist diese Situation auch nicht kritisch. Erst aufgrund der Unsicherheit 
über die zukünftige Bewegung der Verkehrsteilnehmer, welche sich im Mindestabstand $d_{\text {min }}$ ausdrückt, entsteht die Kritikalität, welche sich im Kritikalitätsmaß $K_{\text {uns }}$ zeigt.

Da in der vorliegenden Arbeit die Bewegung aller Verkehrsteilnehmer als bekannt vorausgesetzt wird, entfällt die Kritikalität $K_{\text {uns. }}$. 


\section{A.1.4. Weitere Definitionen}

Definition A.1.17: Der Fit-Wert einer Folge $(y)_{i=1 . . n}$ im Vergleich zu einer Referenzfolge $\left(y_{R e f}\right)_{i=1 \ldots n}$ ist:

$$
f i t\left(y, y_{\text {Ref }}\right)=1-\frac{\sqrt{\sum_{i=1}^{n}\left(y_{i}-y_{\text {Ref }, i}\right)^{2}}}{\sqrt{\sum_{i=1}^{n}\left(y_{R e f, i}-\frac{1}{n} \sum_{i=1}^{n} y_{\text {Ref }, i}\right)^{2}}}
$$

mit $y_{\text {Ref }, i} \neq \frac{1}{n} \sum_{i=1}^{n} y_{\text {Ref }, i}$ für ein beliebiges Glied der Folge $\left(y_{\text {Ref }}\right)_{i=1 \ldots n}$.

Definition A.1.18: Die Überschwingweite einer Folge $(y)_{i=1 . . . n}$ über eine Referenzfolge $\left(y_{\text {Ref }}\right)_{i=1 \ldots n}$ ist:

$$
\operatorname{UWE}\left(y, y_{R e f}\right)=\frac{\max (y)-\max \left(y_{R e f}\right)}{\max \left(y_{R e f}\right)}
$$

mit $\max \left(y_{R e f}\right) \neq 0$.

Definition A.1.19: Die relative Abweichung einer Folge $(y)_{i=1 . . . n}$ von einer Referenzfolge $\left(y_{\text {Ref }}\right)_{i=1 \ldots n}$ ist:

$$
r A\left(y, y_{\text {Ref }}\right)=\frac{1}{n} \sum_{i=1}^{n} \frac{y_{i}-y_{R e f, i}}{y_{R e f, i}}
$$

mit $y_{\text {Ref }, i} \neq 0 \forall i=1 \ldots n$.

Definition A.1.20: Der Normalized Root Mean Squared Error (NRMSE) einer Folge $(y)_{i=1 . . n}$ von einer Referenzfolge $\left(y_{\text {Ref }}\right)_{i=1 . . . n}$ ist:

$$
\operatorname{NRMSE}\left(y, y_{R e f}\right)=\frac{\sqrt{\frac{1}{n} \sum_{i=1}^{n}\left(y_{i}-y_{R e f, i}\right)^{2}}}{\frac{1}{n} \sum_{i=1}^{n} y_{R e f, i}}
$$

mit $\sum_{i=1}^{n} y_{R e f, i} \neq 0$ 


\section{A.2. Modellprädiktive Trajektorienscharregelung}

Der MPTSA erweckt den Eindruck einer speziellen Lösung, dessen grobe Stellgrößendiskretisierung nicht allgemeingültig angewendet werden kann. Ein Nachweis der Allgemeingültigkeit ist mit hohem Aufwand verbunden und sollte Gegenstand weiterer Forschungsvorhaben sein. Anhand einer einfachen Regelstrecke soll hier im Folgenden beispielhaft gezeigt werden, wie das Verfahren auf eine andere Regelstrecke angewendet werden kann und welche Ergebnisse sich erzielen lassen. Der Begriff Model Predictive Trajectory Set Control (MPTSC) ist dann treffender. Als Beispielsystem wird ein lineares schwingfähiges System zweiter Ordnung ausgewählt. Die Regelstrecke wird mit dem linearen Zustandsraummodell der Form

$$
\dot{\mathbf{x}}_{\mathrm{BS}}=\mathbf{A}_{\mathrm{BS}} \mathbf{x}_{\mathrm{BS}}+\mathbf{b}_{\mathrm{BS}} \mathbf{u}_{\mathrm{BS}}, y_{\mathrm{BS}}=\mathbf{c}_{\mathrm{BS}}^{\mathrm{T}} \mathbf{x}_{\mathrm{BS}}
$$

beschrieben. Die Zustandsdifferentialgleichungen sind:

$$
\left[\begin{array}{c}
\dot{x}_{\mathrm{BS}} \\
\ddot{x}_{\mathrm{BS}}
\end{array}\right]=\left[\begin{array}{cc}
0 & 1 \\
-1 / T_{\mathrm{BS}}^{2} & -2 d_{\mathrm{BS}} / T_{\mathrm{BS}}
\end{array}\right]\left[\begin{array}{c}
x_{\mathrm{BS}} \\
\dot{x}_{\mathrm{BS}}
\end{array}\right]+\left[\begin{array}{c}
0 \\
1 / T_{\mathrm{BS}}^{2}
\end{array}\right] u_{\mathrm{BS}}
$$

und die Ausgangsgleichung lautet:

$$
y_{\mathrm{BS}}=\left[\begin{array}{ll}
1 & 0
\end{array}\right]\left[\begin{array}{c}
x_{\mathrm{BS}} \\
\dot{x}_{\mathrm{BS}}
\end{array}\right]
$$

Die Parameter haben die Werte $T_{\mathrm{BS}}=1 \mathrm{~s}$ und $d_{\mathrm{BS}}=0,5$. Hinzu kommt die (nichtlineare) Stellgrößenbeschränkung $u_{\mathrm{BS} \text {, min }}<u_{\mathrm{BS}}<u_{\mathrm{BS} \text {,max }}$. Es handelt sich um eine Regelstrecke mit Ausgleich, sodass bei der Anwendung des MPTSC mit einer festen Diskretisierung der Stellgröße im Allgemeinen eine bleibende Regelabweichung resultiert. Es wird daher die adaptive Diskretisierung verwendet. Wird als Referenzregler ein Zustandsregler mit konstantem Rückführvektor $\mathbf{k}_{\mathrm{BS}}$ eingesetzt, so ergibt sich für lineare Systeme ein nach Riccati ausgelegter Zustandsregler als verlaufs- und verbrauchsoptimale Lösung. Er dient folglich als Referenz, um den MPTSC zu evaluieren. Der Reglerentwurf nach Riccati minimiert das Lagrangesche Gütemaß

$$
J_{\text {Lag }}=\int_{0}^{\infty}\left[\mathbf{x}_{\mathrm{BS}}^{\mathrm{T}} \mathbf{Q}_{\text {Ric }} \mathbf{x}_{\mathrm{BS}}+\mathbf{u}_{\mathrm{BS}}^{\mathrm{T}} \mathbf{R}_{\text {Ric }} \mathbf{u}_{\mathrm{BS}}\right] \mathrm{d} t
$$

und liefert das Reglergesetz

$$
u=-\mathbf{k}_{\mathrm{BS}} \mathbf{x}_{\mathrm{BS}}+M_{\mathrm{BS}} w_{\mathrm{BS}}
$$

Das Führungsfilter $M_{\mathrm{BS}}$ wird auf stationäre Genauigkeit ausgelegt:

$$
M_{\mathrm{BS}}=\left(\mathbf{c}_{\mathrm{BS}}^{\mathrm{T}}\left(\mathbf{b}_{\mathrm{BS}} \mathbf{k}_{\mathrm{BS}}^{\mathrm{T}}-\mathbf{A}_{\mathrm{BS}}\right)^{-1} \mathbf{b}_{\mathrm{BS}}\right)^{-1} .
$$

Beim MPC wird dieses Gütemaß durch einen endlichen Horizont $n_{\mathrm{p}}$ bzw. $T_{\mathrm{pr}}$ approximiert. Der MPTSC stellt eine gröbere Approximation dar, da neben dem zeitlich begrenzten Prädiktionshorizont noch die suboptimale Lösung durch die Stellgrößendiskretisierung hinzukommt. Zudem wird die Approximation nicht genauer je länger 
der Prädiktionshorizont wird, wie es beim MPC der Fall ist. Im Folgenden wird der MPTSC mit dem Riccati-Regler verglichen und anhand dessen evaluiert. Die positiv semidefiniten Gewichtsmatritzen $\mathbf{Q}_{\text {Ric }}$ und $\mathbf{R}_{\text {Ric }}$ werden zunächst als Einheitsmatritzen gewählt. Anschließend wird das Element $q_{\text {Ric,11 }}$ erhöht, sodass der Stellbereich vollkommen ausgenutzt wird. Es ergibt sich die Wahl:

$$
\mathbf{Q}_{\text {Ric }}=\left(\begin{array}{cc}
100 & 0 \\
0 & 1
\end{array}\right), \mathbf{R}_{\text {Ric }}=1
$$

In Abbildung A.1 ist die Sprungantwort des geschlossenen Regelkreises bei Verwendung des MPTSC mit einem Prädiktionshorizont von $T_{\mathrm{pr}}=T_{\mathrm{BS}}$ und des Riccati-Reglers mit diesen Gewichtungsmatritzen und des MPTSC mit optimierten Gewichten dargestellt. Es ist zu erkennen, dass der Riccati-Regler die Stellgrößenbeschränkung
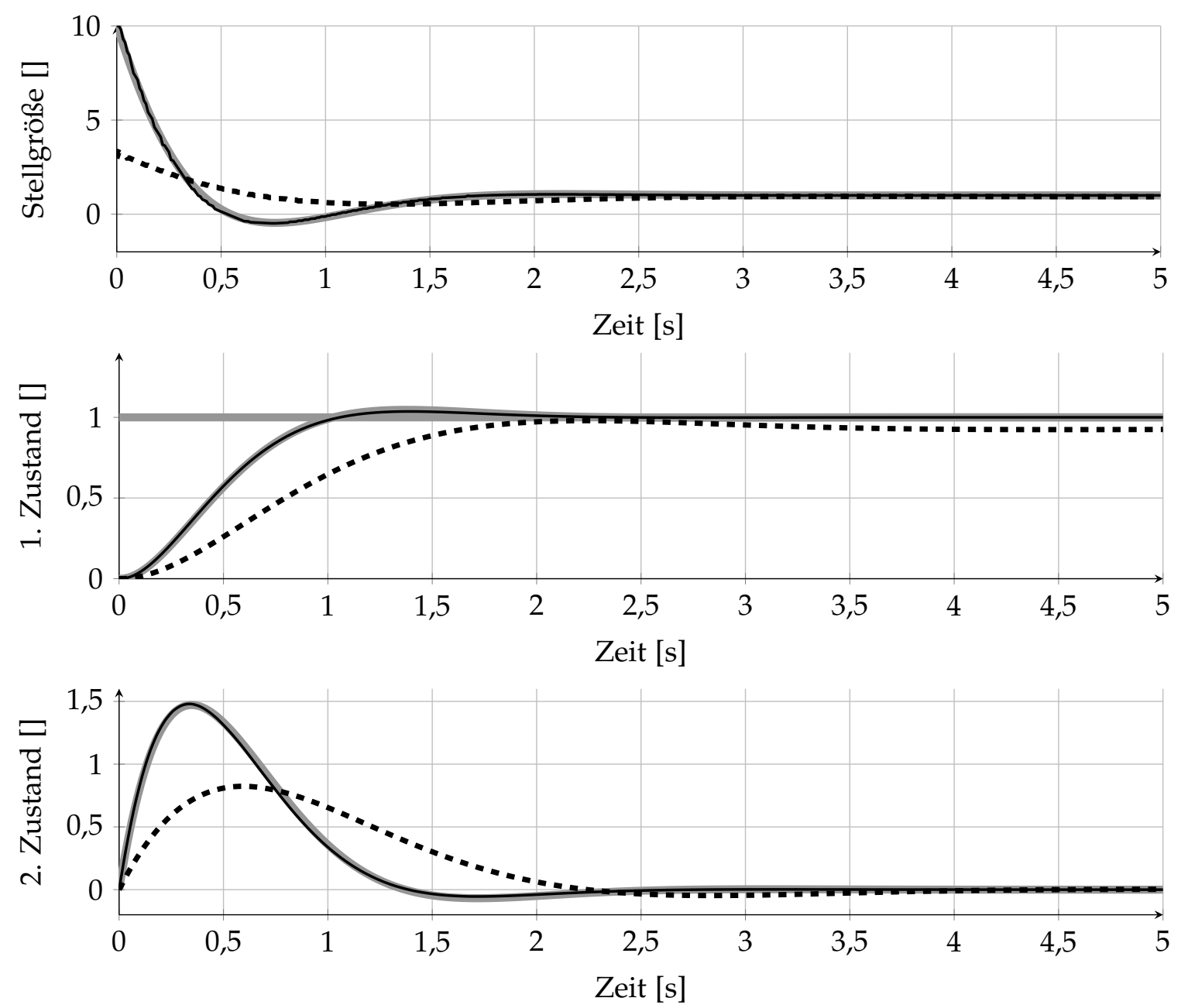

Abbildung A.1.: Vergleich zwischen dem Riccati-Regler und dem MPTSC. Riccati-Regler: grau und durchgzogen, MPTSC mit gleichen Gewichten: schwarz und gestrichelt, MPTSC mit optimierten Gewichten: schwarz und durchgezogen.

von $u_{\mathrm{BS} \text {,max }}=-u_{\mathrm{BS}, \min }=10$ ausnutzt und, mit einem für viele technische Anwendungen akzeptablen Kompromiss zwischen Ausregelzeit und Überschwingweite, die Regelabweichung ohne stationäre Abweichung ausgleicht. Dies ist möglich, da das Streckenmodell der Regelstrecke exakt entspricht und das Führungsfilter entsprechend 
ausgelegt wurde. Die Sprungantwort des MPTSC zeigt ein deutlich abweichendes Verhalten. Die Anstiegszeit ist langsamer und es bleibt (trotz adaptiver Stellgrößendiskretisierung) eine stationäre Regelabweichung. Diese resultiert, sobald das Gewicht

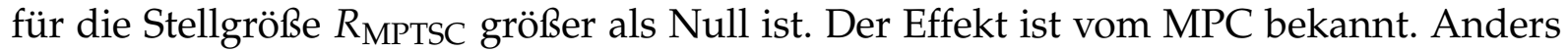
als der MPC stellt der MPTSC keine gute Näherung des Riccati-Reglers dar, wenn das gleiche Gütemaß verwendet wird. Im Folgenden wird gezeigt, wie durch eine andere Wahl der Gewichtungsmatritzen und des Prädiktionshorizontes die Sprungantwort des MPTSC an die des Riccati-Reglers angenähert werden kann. Da die Stellgrößenbeschränkung bei der Diskretisierung explizit berücksichtigt wird, ist ein zusätzlicher verbrauchsoptimaler Term hier nicht notwendig, sodass keine bleibende Regelabweichung zu erwarten ist. Alternativ kann die Regelstrecke um einen Integrator ergänzt werden. Die drei Parameter $q_{\mathrm{MPTSC}, 11}, q_{\mathrm{MPTSC}, 22}$ und $T_{\mathrm{pr}}$ werden durch einen genetischen Algorithmus optimiert, wobei als Referenz die Sprungantwort des nach Riccati geregelten Systems ist. Das Gütemaß lautet:

$$
J_{\mathrm{MR}}=\sum_{i=1}^{n}\left(y_{\mathrm{Ric}, i}-y_{\mathrm{MPTSC}, i}\right)^{2}
$$

In Abbildung A.1 sind die Sprungantwort und die Stellgrößenverläufe zu erkennen. Die Linien verlaufen nahezu deckungsgleich. Die zuvor auftretende stationäre Regelabweichung tritt, wie zu erwarten war, nicht auf. Die Auswertung des Lagrangeschen Gütemaßes basierend auf den Gewichtungsmatritzen des Riccati-Reglers auf dem gezeigten Ausschnitt ergibt für den MPTSC im Vergleich zum Riccati-Regler eine relative Abweichung nach Definition A.1.19

$$
d_{\text {Lag }}\left(J_{\text {Lag,MPTSC}}, J_{\text {Lag,Ric }}\right)=0,05 \% \text {. }
$$

Der Fit-Wert der beiden Sprungantworten beträgt nach Definition A.1.17

$$
\text { fit }\left(y_{\text {MPTSC }}, y_{\text {Ric }}\right)=98,28 \% \text {. }
$$

Wichtige Kennzahlen zu den Sprungantworten sind in Tabelle A.1 zusammengefasst. Die Sprungantwort des nach Riccati geregelten Systems kommt dem MPTSC entgegen,

Tabelle A.1.: Zusammenfassung der Kennwerte zu Abbildung A.1.

\begin{tabular}{|c|c|c|c|}
\hline Reglervariante & $U W E[\%]$ & $d_{\text {Lag }}[\%]$ & fit $[\%]$ \\
\hline Riccati & 4,64 & 0 & 100 \\
\hline MPTSC & $-2,04$ & 31,67 & 34,66 \\
\hline MPTSC optimiert & 3,64 & 0,05 & 98,28 \\
\hline
\end{tabular}

da dieser zu Sprungantworten mit (leichtem) Überschwingen neigt. Im Folgenden werden die Gewichtungsmatritzen des Riccati-Reglers daher mit einem genetischen Algorithmus optimiert, um eine Sprungantwort ohne bzw. mit vernachlässigbarem Überschwingen zu erhalten. Das Gütemaß wird wie folgt gewählt:

$$
J_{\text {Ric }}=\sum_{i=1}^{n}\left[\left(g_{\mathrm{y}}\left(y_{\text {soll }}-y_{\text {Ric }}\right)\right)^{2}+\left(g_{\mathrm{u}} u_{\text {Ric }}\right)^{2}\right]
$$


mit

$$
g_{y}= \begin{cases}1 & \text { wenn }\left(y_{\text {soll }}-y_{\text {Ric }}\right)>0 \\ 10 & \text { sonst }\end{cases}
$$

und

$$
g_{u}= \begin{cases}0 & \text { wenn } u_{\mathrm{BS}, \min }<u_{\text {Ric }}<u_{\mathrm{BS}, \max } \\ 10000 & \text { sonst }\end{cases}
$$

Das Gütemaß verhindert, dass die Stellgrößenbegrenzung verletzt wird und bestraft negative Regelabweichungen (Überschwingen) deutlich stärker als positive. Anschließend wird wiederum versucht, die Sprungantwort mit dem MPTSC zu reproduzieren. In Tabelle A.2 und in Abbildung A.2 ist zu erkennen, dass dies nur unzureichend gelingt. Die relative Abweichung des Lagrangeschen Gütemaßes beträgt $d_{\text {Lag }}=-0,17 \%$

Tabelle A.2.: Zusammenfassung der Kennwerte zu Abbildung A.2.

\begin{tabular}{|c|c|c|c|}
\hline Reglervariante & $U W E[\%]$ & $d_{\mathrm{Lag}}[\%]$ & fit $[\%]$ \\
\hline Riccati & 1,01 & 0 & 100 \\
\hline MPTSC optimiert & 3,22 & $-0,17$ & 96,61 \\
\hline MPTSC Gütemaßanpassung & 0,53 & 0,02 & 99,19 \\
\hline
\end{tabular}

und der Fit-Wert $f$ it $=96,61 \%$. Insbesondere kommt der MPTSC der Forderung nach einer Sprungantwort ohne Überschwingen nicht nach. Der Riccati-Regler erzeugt eine Überschwingweite nach Definition A.1.18 von

$$
U W E\left(y_{\text {Ric }}, y_{\text {soll }}\right)=1,01 \%,
$$

während der MPTSC ein Überschwingen von $U W E=3,22 \%$ aufweist. Die Reproduktion der Sprungantwort ist daher nicht gelungen. Es ist aber anzumerken, dass der MPTSC einen geringfügig niedrigeren Wert des Lagrangeschen Gütemaßes liefert und damit nicht ungeeignet ist. Es ist jedoch möglich die Reproduktion des Riccati-Reglers zu verbessern, indem das Gütemaß des MPTSC strukturell anders gewählt wird.

$$
\begin{array}{r}
J_{\text {MPTSC }}=\sum_{k=1}^{n_{\mathrm{p}}}\left[\left(g_{\mathrm{y}}\left(y_{\text {soll }}-y_{\text {MPTSC }}\right)\right)^{2}+\left(g_{\dot{y}} \dot{y}\right)^{2}\right] \\
g_{\mathrm{y}}= \begin{cases}g_{\mathrm{y} 1} & \text { wenn }\left(y_{\text {soll }}-y_{\text {MPTSC }}\right)>0 \wedge y_{\text {soll }}>y_{k=0} \\
g_{\mathrm{y} 1} & \text { wenn }\left(y_{\text {MPTSC }}-y_{\text {MPTSC }}\right)<0 \wedge y_{\text {soll }}<y_{k=0} \\
g_{\mathrm{y} 2} & \text { sonst }\end{cases}
\end{array}
$$

Es werden nun vier Parameter mit dem genetischen Algorithmus optimiert $\left(g_{\mathrm{y} 1}, g_{\mathrm{y} 2}, g_{\dot{y}}\right.$ und $T_{\text {pr }}$ ), um die Sprungantwort des nach Riccati geregelten Systems zu reproduzieren. Das Ergebnis ist in Abbildung A.2 zu erkennen. Die relative Abweichung des Lagrangenschen Gütmaßes beträgt $d_{\text {Lag }}=0,02 \%$ und die Überschwingweite $U W E=0,53 \%$. Durch die gesonderte Gewichtung von Überschwingern, kommt es beim Schneiden des Sollwertes zu kleinen Spitzen im Stellgrößenverlauf, welche je nach Anwendung unterschiedlich zu bewerten sind. Tabelle A.2 fasst die Kennzahlen 

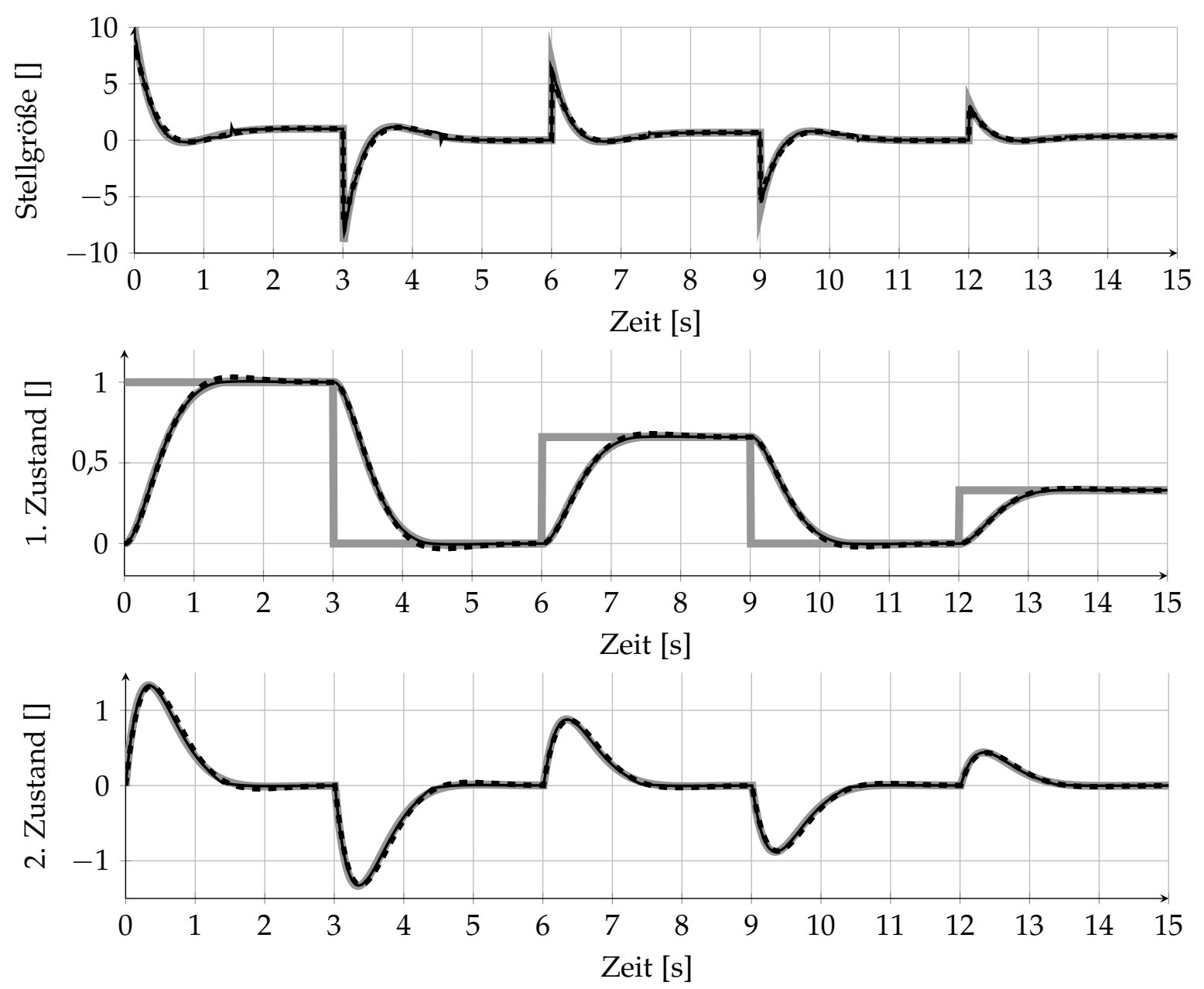

Abbildung A.2.: Vergleich zwischen dem Riccati-Regler und dem MPTSC. Riccati-Regler: grau und durchgezogen, MPTSC mit gleichen Gütemaß: schwarz und gestrichelt, MPTSC mit angepasstem Gütemaß: schwarz und durchgezogen.

zusammen. Insgesamt ist es (in diesem Anwendungsfall) gelungen durch die freie Gestaltungsmöglichkeit des Gütemaßes beim MPTSC, den Nachteil der Suboptimalität aufzuwiegen. Abschließend wird der Riccati-Regler manuell so eingestellt, dass ein stärker gedämpftes Übergangsverhalten entsteht. Abbildung A.3 zeigt die Sprungantwort, welche ebenfalls gut reproduziert wird. Der Fit-Wert beträgt 98,24\% und die relative Abweichung des Lagrangeschen Gütemaßes beträgt $d_{\text {Lag }}=0,15 \%$. Wichtige Kennzahlen werden in Tabelle A.3 zusammengefasst. Abschließend ist festzustellen, dass die Suboptimalität in diesem Anwendungsbeispiel vernachlässigbar ist und die freie Gestaltungsmöglichkeit des Gütemaßes die Einstellung des Übergangsverhaltens in einem weiten Bereich erlaubt. Der hier beispielhaft durchgeführte Vergleich beweist nicht, dass der MPTSC für jede Regelstrecke anwendbar ist und darüber hinaus eine zufriedenstellende Regelungsgüte aufweist. Das Ergebnis des Vergleichs lässt aber darauf schließen, dass zumindest einige (technisch relevante) Regelungsaufgaben mit dem MPTSC gelöst werden können. Darüber hinaus erscheint es naheliegend, dass sich bei einigen Anwendungen, aufgrund der modellprädiktiven Eigenschaften des Reglers, sehr gute Ergebnisse erzielen lassen. Die Grenzen und Möglichkeiten des MPTSC sollten durch weitere Forschungsarbeiten untersucht werden. 

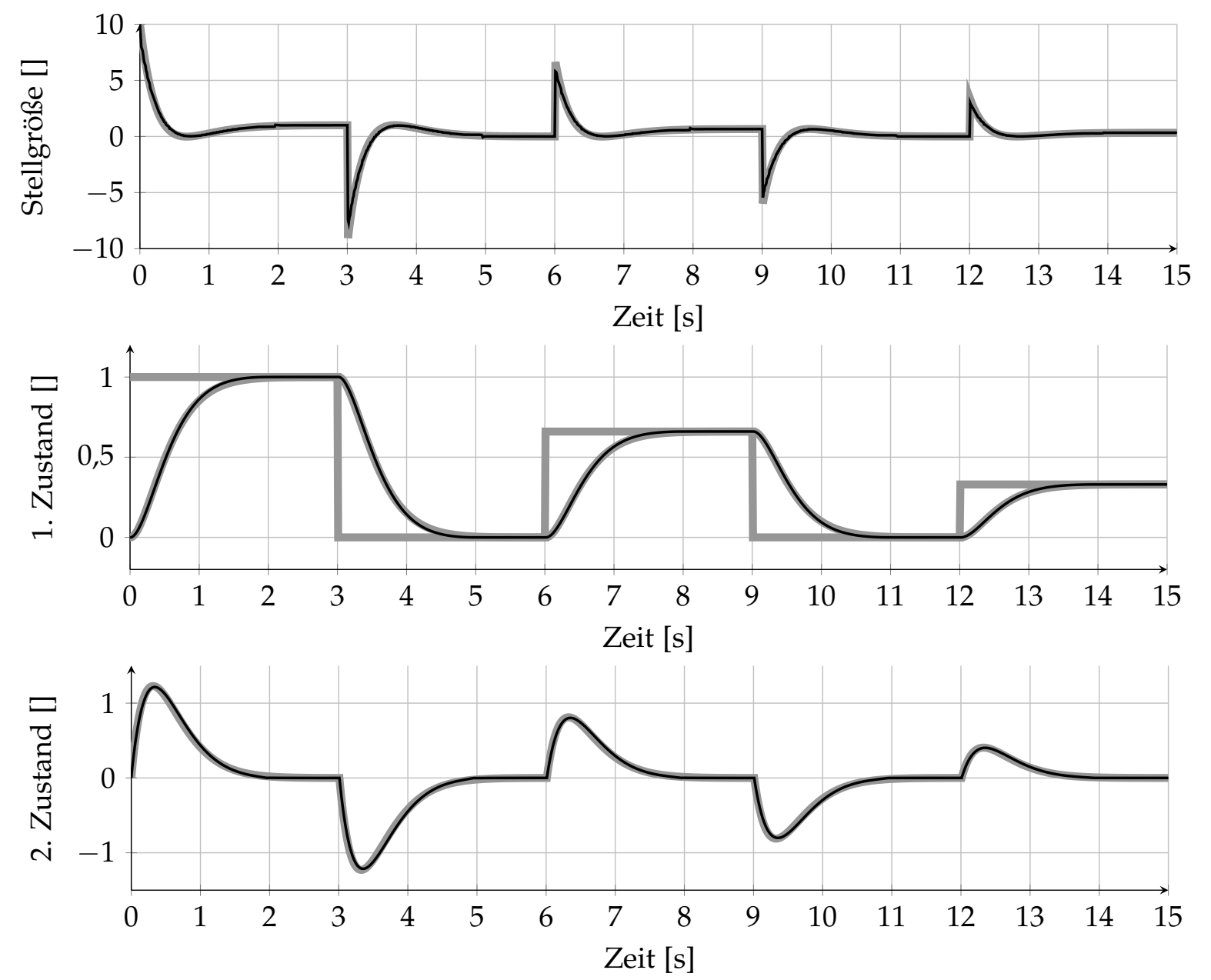

Abbildung A.3.: Vergleich zwischen dem Riccati-Regler und dem MPTSC bei stark gedämpfter Sprungantwort. Riccati-Regler: grau und durchgzogen, MPTSC: schwarz und durchgezogen.

Tabelle A.3.: Zusammenfassung der Kennwerte zu Abbildung A.3

\begin{tabular}{|c|c|c|c|}
\hline Reglervariante & $U W E[\%]$ & $d_{\mathrm{Lag}}[\%]$ & fit $[\%]$ \\
\hline Riccati & 0,01 & 0 & 100 \\
\hline MPTSC optimiert & 0,04 & 0,15 & 98,24 \\
\hline
\end{tabular}




\section{Literatur}

[Ack+14] C. Ackermann, R. Isermann, S. Min und C. Kim. „Collision avoidance with automatic braking and swerving". In: 19th IFAC World Congress 2014. Bd. 47. 2014, S. 10694-10699.

[Ack+15] C. Ackermann, J. Bechtloff und R. Isermann. „Collision avoidance with combined braking and steering". In: 6th International Munich Chassis Symposium 2015. Hrsg. von P. E. Pfeffer. Proceedings. Wiesbaden: Springer Vieweg, 2015, S. 199-213.

[And+09] S. J. Anderson, S. C. Peters, K. D. Iagnemma und T. E. Pilutti. „A unified approach to semi-autonomous control of passenger vehicles in hazard avoidance scenarios". In: 2009 IEEE International Conference on Systems, Man and Cybernetics - SMC. 2009, S. 2032-2037.

[And+12] S. J. Anderson, S. B. Karumanchi und K. Iagnemma. „Constraint-based planning and control for safe, semi-autonomous operation of vehicles". In: 2012 IEEE Intelligent Vehicles Symposium (IV). 2012, S. 383-388.

[Bau+12] E. Bauer, F. Lotz, M. Pfromm, M. Schreier, B. Abendroth, S. Cieler, A. Eckert, A. Hohm, S. Lüke, P. Rieth, V. Willert und J. Adamy. „PRORETA 3: An Integrated Approach to Collision Avoidance and Vehicle Automation". In: at - Automatisierungstechnik Jg. 60, Nr. 12 (2012), S. 755-765.

[Bau+13] E. Bauer und U. Konigorski. „Ein modellprädiktiver Querplanungsansatz zur Kollisionsvermeidung: Modellierung von Potentialfeldern der statischen und dynamischen Umgebung innerhalb eines Kostenfunktionals". In: VDI AUTOREG 2013. Bd. VDI Berichte 2196. 2013, S. 195-206.

[Bev+10] G. P. Bevan, H. Gollee und J. O'Reilly. „Trajectory generation for road vehicle obstacle avoidance using convex optimization ". In: Proceedings of the Institution of Mechanical Engineers, Part D: Journal of Automobile Engineering Jg. 224, Nr. 4 (2010), S. 455-473.

[Bev08] G. P. Bevan. „Development of a Vehicle Dynamics Controller for Obstacle Avoidance“. Dissertation. University of Glasgow, 2008.

[Bra+07] T. Brandt, T. Sattel und M. Bohm. "Combining haptic human-machine interaction with predictive path planning for lane-keeping and collision 
avoidance systems“. In: 2007 IEEE Intelligent Vehicles Symposium. 2007, S. 582-587.

[Bra07] T. Brandt. „A Predictive Potential Field Concept for Shared Vehicle Guidance“. Dissertation. Universität Paderborn, 2007.

[Buß13] M. Buß. „Modellprädiktive Regelung der Querdynamik von Kraftfahrzeugen entlang vorgegebener Bahnen im fahrphysikalischen Grenzbereich". Masterarbeit. Technische Universität Dortmund, 2013.

[Cho+10] J. Choi, R. E. Curry und G. H. Elkaim. „Continuous Curvature Path Generation Based on Bezier Curves for Autonomous Vehicles". In: International Journal of Applied Mathematics Jg. 40, Nr. 2 (2010), S. 91-101.

[Cho+11] J. Choi, K. Kim und K. Yi. „Emergency driving support algorithm with steering torque overlay and differential braking". In: 2011 14th International IEEE Conference on Intelligent Transportation Systems - (ITSC 2011). 2011, S. $1433-1439$.

[Cho+12] C. Choi, Y. Kang und S. Lee. „Emergency collision avoidance maneuver based on nonlinear model predictive control“. In: 2012 IEEE International Conference on Vehicular Electronics and Safety (ICVES 2012). 2012, S. 393-398.

[Cho+14] J. Choi, K. Yi, J. Suh und B. Ko. „Coordinated Control of Motor-Driven Power Steering Torque Overlay and Differential Braking for Emergency Driving Support“. In: IEEE Transactions on Vehicular Technology Jg. 63, Nr. 2 (2014), S. 566-579.

[Del+10] M. Della Penna, M. M. van Paassen, D. A. Abbink, M. Mulder und M. Mulder. „Reducing steering wheel stiffness is beneficial in supporting evasive maneuvers". In: 2010 IEEE International Conference on Systems, Man and Cybernetics - SMC. 2010, S. 1628-1635.

[Des15] Destatis. Verkehrsunfälle: Zeitreihen. Statistisches Bundesamt. Wiesbaden, 2015.

[Din+10] P. Dingle und L. Guzzella. „Optimal emergency maneuvers on highways for passenger vehicles with two- and four-wheel active steering". In: 2010 American Control Conference (ACC 2010). 2010, S. 5374-5381.

[Eid+07] A. Eidehall, J. Pohl, F. Gustafsson und J. Ekmark. „Toward Autonomous Collision Avoidance by Steering". In: IEEE Transactions on Intelligent Transportation Systems Jg. 8, Nr. 1 (2007), S. 84-94.

[Eid11] A. Eidehall. „Multi-target threat assessment for automotive applications". In: 2011 14th International IEEE Conference on Intelligent Transportation Systems - (ITSC 2011). 2011, S. 433-438.

[Esk+08] A. Eskandarian und D. Soudbakhsh. „Enhanced Active Steering System for Collision Avoidance Maneuvers“. In: 2008 11th International IEEE Conference on Intelligent Transportation Systems - (ITSC 2008). 2008, S. 1049-1054. 
[Fal+07] P. Falcone, F. Borrelli, J. Asgari, H. E. Tseng und D. Hrovat. „Predictive Active Steering Control for Autonomous Vehicle Systems". In: IEEE Transactions on Control Systems Technology Jg. 15, Nr. 3 (2007), S. 566-580.

[Fer+11] D. Fernandez Llorca, V. Milanes, I. Parra Alonso, M. Gavilan, I. Garcia Daza, J. Perez und M. Á. Sotelo. „Autonomous Pedestrian Collision Avoidance Using a Fuzzy Steering Controller". In: IEEE Transactions on Intelligent Transportation Systems Jg. 12, Nr. 2 (2011), S. 390-401.

[Fer+16] J. Ferdinand und B. Yi. „Trajetory Planning for Collision Avoidance in Urban Area". In: 2016 IEEE Intelligent Vehicles Symposium. 2016, S. 202-207.

[Fra+13] J. V. Frasch, A. Gray, M. Zanon, H. J. Ferreau, S. Sager, F. Borrelli und M. Diehl. „An Auto-generated Nonlinear MPC Algorithm for Real-Time Obstacle Avoidance of Ground Vehicles". In: 2013 European Control Conference (ECC). 2013, S. 4136-4141.

[Fu+15] M. Fu, W. Song, Y. Yi und M. Wang. „Path Planning and Decision Making for Autonomous Vehicle in Urban Environment". In: 2015 IEEE 18th International Conference on Intelligent Transportation Systems - (ITSC 2015). 2015, S. 686-692.

[Gao+12] Y. Gao, A. Gay, J. V. Frasch, T. Lin, E. Tseng, J. K. Hedrick und F. Borrelli. "Spatial Predictive Control for Agile Semi-Autonomous Ground Vehicles". In: 11th International Symposium on Advanced Vehicle Control. 2012, o.S.

[Geh+01] S. K. Gehrig und F. J. Stein. "Elastic bands to enhance vehicle following“. In: 2001 IEEE Intelligent Transportation Systems Conference. Proceedings. 2001, S. 597-602.

[Gor+14] T. Gordon und Y. Gao. „A flexible hierarchical control method for optimal collision avoidance". In: 2014 16th International Conference on Mechatronics Mechatronika (ME). 2014, S. 318-324.

[Göt+15] C. Götte, M. Keller, C. Haß, K.-H. Glander, A. Seewald und T. Bertram. „A Model Predictive Combined Planning and Control approach for Guidance of Automated Vehicles". In: 2015 IEEE International Conference on Vehicular Electronics and Safety (ICVES). 2015, S. 69-74.

[Göt+16a] C. Götte, M. Keller, C. Rösmann, T. Nattermann, C. Haß, K.-H. Glander, A. Seewald und T. Bertram. "A Real-Time Capable Model Predictive Approach to Lateral Vehicle Guidance“. In: 2016 IEEE 19th International Conference on Intelligent Transportation Systems (ITSC 2016). 2016, S. 19081913.

[Göt+16b] C. Götte, M. Keller, C. Haß und T. Bertram. „Modellprädiktive Planung und Regelung im Anwendungsfall kritischer Verkehrssituationen". In: ATZ - Automobiltechnische Zeitschrift Jg. 118, Nr. 9 (2016), S. 68-73.

[Göt14] C. Götte. „Integration eines Fahrdynamikmodells in das Timed Elastic Band Verfahren zur Durchführung optimaler Bremsausweichmanöver". Masterarbeit. Technische Universität Dortmund, 2014. 
[Har14] H. Hartmann. „Entwicklung und Untersuchung eines modellprädiktiven Trajektorienscharplanungs- und Fahrzeugquerregelungsverfahrens zur Unfallvermeidung im Straßenverkehr durch Notausweichmanöver". Bachelorarbeit. Technische Universität Dortmund, 2014.

[Has+12] M. Hassanzadeh, M. Lidberg, M. Keshavarz und L. Bjelkeflo. „Path and speed control of a heavy vehicle for collision avoidance manoeuvres". In: 2012 IEEE Intelligent Vehicles Symposium (IV). 2012, S. 129-134.

[Hay+12] R. Hayashi, J. Isogai, P. Raksincharoensak und M. Nagai. „Autonomous collision avoidance system by combined control of steering and braking using geometrically optimised vehicular trajectory“. In: Vehicle System Dynamics Jg. 50, Nr. sup1 (2012), S. 151-168.

[Haß+14] C. Haß, T. Bertram und M. Keller. „Steuergerät und Verfahren für eine Notfall-Lenkunterstützungsfunktion“. Pat. WO 2014/195087 A1; DE 10 2013009252 A1; US 2016/0280265 A1; CN 105813914 A. 2014.

[Haß05] C. Haß. „Modellbasierter Systementwurf eines reversiblen mechatronischen Gurtstraffers“. Dissertation. Universität Duisburg-Essen, 2005.

[Her+15] S. Herrmann, W. Utschick, M. Botsch und F. Keck. „Supervised Learning via Optimal Control Labeling for Criticality Classification in Vehicle Active Safety". In: 2015 IEEE 18th International Conference on Intelligent Transportation Systems - (ITSC 2015). 2015, S. 2024-2031.

[Hil+03] J. Hilgert, K. Hirsch, T. Bertram und M. Hiller. „Emergency path planning for autonomous vehicles using elastic band theory". In: 2003 IEEE/ASME International Conference on Advanced Intelligent Mechatronics (AIM 2003). 2003, S. 1390-1395.

[Hil05] J. Hilgert. „Anwendung der Ähnlichkeitstheorie zur experimentellen Eigenschaftsabsicherung eines Bahnplanungsverfahrens für Fahrzeugführungssysteme“. Dissertation. Universität Duisburg-Essen, 2005.

[Hom+17] A. Homann, M. Keller, C. Götte, K.-H. Glander, C. Haß und T. Bertram. „Kinodynamic Trajectory Optimization for Real-Time Vehicle Guidance: Kinodynamische Trajektorien Optimierung zur Echtzeit Fahrzeugführung". In: Automotive meets Electronics 2017. 2017, S. 14-19.

[Hom13] A. Homann. „Beschreibung von optimalen Bremsausweichtrajektorien in kritischen Verkehrsituationen". Bachelorarbeit. Technische Universität Dortmund, 2013.

[Hom15] A. Homann. „Ein Verfahren zur Fahrzeugführung mittels echtzeitfähiger Trajektorienoptimierung und Fahrdynamikregelung“. Masterarbeit. Technische Universität Dortmund, 2015.

[Hun+08] F. von Hundelshausen, M. Himmelsbach, F. Hecker, A. Mueller und H.-J. Wuensche. „Driving with tentacles: Integral structures for sensing and motion“. In: Journal of Field Robotics Jg. 25, Nr. 9 (2008), S. 640-673. 
[Ise+12] R. Isermann, R. Mannale und K. Schmitt. „Collision-avoidance systems PRORETA: Situation analysis and intervention control“. In: Control Engineering Practice Jg. 20, Nr. 11 (2012), S. 1236-1246.

[Ise06] R. Isermann. Fahrdynamikregelung: Modellbildung, Fahrerassistenzsysteme, Mechatronik. Wiesbaden: Friedr. Vieweg \& Sohn Verlag, 2006.

[Ito+12] M. Itoh, T. Inagaki und H. Tanaka. „Haptic steering direction guidance for pedestrian-vehicle collision avoidance“. In: 2012 IEEE International Conference on Systems, Man and Cybernetics - SMC. 2012, S. 3327-3332.

[Ito+13] M. Itoh, T. Horikome und T. Inagaki. „Effectiveness and driver acceptance of a semi-autonomous forward obstacle collision avoidance system". In: Applied Ergonomics Jg. 44, Nr. 5 (2013), S. 756-763.

[Iwa+14] K. Iwano, P. Raksincharoensak und M. Nagai. „A Study on Shared Control between the Driver and an Active Steering Control System in Emergency Obstacle Avoidance Situations". In: 19th IFAC World Congress 2014. Bd. 47. 2014, S. 6338-6343.

[Kae+09] N. Kaempchen, B. Schiele und K. Dietmayer. „Situation Assessment of an Autonomous Emergency Brake for Arbitrary Vehicle-to-Vehicle Collision Scenarios". In: IEEE Transactions on Intelligent Transportation Systems Jg. 10, Nr. 4 (2009), S. 678-687.

[Kam+36] W. Kamm, O. Hoffmeister und L. Huber. Das Kraftfahrzeug: Betriebsgrundlagen, Berechnung, Gestaltung und Versuch. Berlin: Springer, 1936.

[Kar08] S. Karrenberg. "Zur Erkennung unvermeidbarer Kollisionen mit Hilfe von Stellvertretertrajektorien“. Dissertation. Technische Universität CaroloWilhelmina zu Braunschweig, 2008.

[Kat+13] A. Katriniok, J. P. Maschuw, F. Christen, L. Eckstein und D. Abel. „Optimal Vehicle Dynamics Control for Combined Longitudinal and Lateral Autonomous Vehicle Guidance“. In: 2013 European Control Conference (ECC). 2013, S. 974-979.

[Kat+14] D. I. Katzourakis, J. C. F. de Winter, M. Alirezaei, M. Corno und R. Happee. "Road-Departure Prevention in an Emergency Obstacle Avoidance Situation". In: IEEE Transactions on Systems, Man, and Cybernetics: Systems Jg. 44, Nr. 5 (2014), S. 621-629.

[Kat+15] C. Katrakazas, M. Quddus, W.-H. Chen und L. Deka. „Real-time motion planning methods for autonomous on-road driving: State-of-the-art and future research directions". In: Transportation Research Part C: Emerging Technologies Jg. 60 (2015), S. 416-442.

[Keh+06] S. Kehl, W.-D. Pölsler und M. Zeitz. „Querregelung eines Versuchsfahrzeugs entlang vorgegbener Bahnen“. In: AUTOREG 2006. Bd. VDI Berichte NR 1931. 2006, S. 99-108. 
[Keh+07] S. Kehl, W.-D. Pölsler und M. Zeitz. "Querregelung eines Versuchsfahrzeugs entlang vorgegebener Bahnen (Lateral Control of a Test Vehicle along Predefined Paths)“. In: at - Automatisierungstechnik Jg. 55, Nr. 6 (2007), S. 306-313.

[Keh07] S. Kehl. „Querregelung eines Versuchsfahrzeugs entlang vorgegebener Bahnen". Dissertation. Universität Stuttgart, 2007.

[Kel+11] C. G. Keller, T. Dang, H. Fritz, A. Joos, C. Rabe und D. M. Gavrila. „Active Pedestrian Safety by Automatic Braking and Evasive Steering". In: IEEE Transactions on Intelligent Transportation Systems Jg. 12, Nr. 4 (2011), S. 1292 1304.

[Kel+13] M. Keller, C. Haß, A. Seewald und T. Bertram. „Ein Regelungskonzept für eine Notausweichassistenz: Trajektorienfolgeregelung für den querdynamischen Grenzbereich“. In: VDI AUTOREG 2013. Bd. VDI Berichte Nr. 2196. 2013, S. 363-372.

[Kel+14a] M. Keller, C. Hass, A. Seewald und T. Bertram. „Driving simulator study on an emergency steering assist". In: 2014 IEEE International Conference on Systems, Man and Cybernetics - SMC. 2014, S. 3008-3013.

[Kel+14b] M. Keller, F. Hoffmann, T. Bertram, C. Hass und A. Seewald. „Planning of Optimal Collision Avoidance Trajectories with Timed Elastic Bands". In: 19th IFAC World Congress 2014. Bd. 47. 2014, S. 9822-9827.

[Kel+14c] M. Keller, C. Hass, A. Seewald und T. Bertram. „Sensitivitätsanalyse der die Fahrzeugdynamik beeinflussenden Systemparameter". In: Automotive meets Electronics 2014. 2014, S. 141-146.

[Kel+15a] M. Keller, C. Hass, A. Seewald und T. Bertram. „A Model Predictive Approach to Emergency Maneuvers in Critical Traffic Situations". In: 2015 IEEE 18th International Conference on Intelligent Transportation Systems (ITSC 2015). 2015, S. 369-374.

[Kel+15b] M. Keller, C. Haß, A. Seewald und T. Bertram. „A Vehicle Lateral Control Approach for Collision Avoidance by Emergency Steering Maneuvers". In: 6th International Munich Chassis Symposium 2015. Hrsg. von P. E. Pfeffer. Proceedings. Wiesbaden: Springer Vieweg, 2015, S. 175-197.

[Kel+15c] M. Keller, C. Haß, A. Seewald und T. Bertram. „Ein modellprädiktives Planungs- und Fahrzeugquerregelungsverfahren zur Kollisionsvermeidung durch Notausweichmanöver". In: Automotive meets Electronics 2015. 2015, S. 8-13.

[Kel11] M. Keller. „Beschreibung von Ausweichtrajektorien für eine Bahnfolgeregelung“. Diplomarbeit. Technische Universität Dortmund, 2011.

[Kön+07] L. König, J. Neubeck und J. Wiedemann. „Nichtlineare Lenkregler für den querdynamischen Grenzbereich (Nonlinear Steering Controllers for the Lateral Dynamics Stability Limit)“. In: at - Automatisierungstechnik Jg. 55, Nr. 6 (2007). 
[Kön09] L. König. Ein virtueller Testfahrer für den querdynamischen Grenzbereich. Bd. 43. Schriftenreihe des Instituts für Verbrennungsmotoren und Kraftfahrwesen der Universität Stuttgart. Renningen: expert-Verl., 2009.

[Mad+13] D. Madas, M. Nosratinia, M. Keshavarz, P. Sundström, R. Philippsen, A. Eidehall und K.-M. Dahlen. „On Path Planning Methods for Automotive Collision Avoidance". In: 2013 IEEE Intelligent Vehicles Symposium (IV). 2013, S. 931-937.

[May91] R. Mayr. „Verfahren zur Bahnfolgeregelung für ein automatisch geführtes Fahrzeug". Dissertation. Universität Dortmund, 1991.

[McN+11] M. McNaughton, C. Urmson, J. M. Dolan und J.-W. Lee. „Motion planning for autonomous driving with a conformal spatiotemporal lattice“. In: 2011 IEEE International Conference on Robotics and Automation (ICRA). 2011, S. $4889-4895$.

[Meh+10] E. Mehrjerdian, T. Bertram, A. Gaedke und M. Sprinzl. „Superposition of Steering Torque to Support the Driver in critical Situation". In: 1. Internationales Münchener Fahrwerk-Symposium chassis.tech plus. 2010, o.S.

[Meh15] E. Mehrjerdian. „Potenziale einer Lenkmomentenempfehlung unter fahrdynamischen Gesichtspunkten“. Dissertation. Technische Universität Dortmund, 2015.

[Men+14] L. Menhour, B. d'Andréa Novel, M. Fliess und H. Mounier. „Coupled nonlinear vehicle control: Flatness-based setting with algebraic estimation techniques“. In: Control Engineering Practice Jg. 22 (2014), S. 135-146.

[Mil+12] V. Milanés, J. Pérez, J. Godoy und E. Onieva. „A fuzzy aid rear-end collision warning/avoidance system". In: Expert Systems with Applications Jg. 39, Nr. 10 (2012), S. 9097-9107.

[Mit03] M. Mitschke. Dynamik von Kraftfahrzeugen. 4. [bearb.] Aufl. Berlin: Springer, 2003.

[Mon+08] M. Montemerlo, J. Becker, S. Bhat, H. Dahlkamp, D. Dolgov, S. Ettinger, D. Haehnel, T. Hilden, G. Hoffmann, B. Huhnke, D. Johnston, S. Klumpp, D. Langer, A. Levandowski, J. Levinson, J. Marcil, D. Orenstein, J. Paefgen, I. Penny, A. Petrovskaya, M. Pflueger, G. Stanek, D. Stavens, A. Vogt und S. Thrun. "Junior: The Stanford entry in the Urban Challenge". In: Journal of Field Robotics Jg. 25, Nr. 9 (2008), S. 569-597.

[Mos+12] N. Moshchuk, S.-K. Chen, C. Zagorski und A. Chatterjee. „Optimal braking and steering control for active safety". In: 2012 15th International IEEE Conference on Intelligent Transportation Systems - (ITSC 2012). 2012, S. 17411746.

[Muk+14] M. Mukai und T. Kawabe. „Driver assistance algorithm for automotive collision avoidance using optimization feasibility". In: 19th IFAC World Congress 2014. Bd. 47. 2014, S. 416-421. 
[Pac06] H. B. Pacejka. Tyre and vehicle dynamics. 2nd ed. Oxford: ButterworthHeinemann, 2006.

[Pre+01] C. Preuße, H. Keller und K. J. Hunt. „Fahrzeugführung durch ein Fahrermodell (Trajectory Finding as Part of a Driver Model)“. In: at - Automatisierungstechnik Jg. 49, Nr. 12/2001 (2001).

[Qui+93] S. Quinlan und O. Khatib. „Elastic bands: connecting path planning and control“. In: [1993] IEEE International Conference on Robotics and Automation. 1993 , S. 802-807.

[Rug+15] F. Ruger, V. Nitsch und B. Farber. "Automatic Evasion Seen from the Opposing Traffic - An Investigation with the Vehicle in the Loop“. In: 2015 IEEE 18th International Conference on Intelligent Transportation Systems (ITSC 2015). 2015, S. 1041-1048.

[Rös+12] C. Rösmann, W. Feiten, T. Wösch, F. Hoffmann und T. Bertram. „Trajectory Modification Considering Dynamic Constraints of Autonomous Robots". In: Proceedings of ROBOTIK 2012; 7th German Conference on Robotics. 2012, S. 1-6.

[Rös+13] C. Rösmann, W. Feiten, T. Wösch, F. Hoffmann und T. Bertram. „Efficient trajectory optimization using a sparse model“. In: 2013 European Conference on Mobile Robots (ECMR). 2013, S. 138-143.

[Rös+14] C. Rösmann, F. Hoffmann und T. Bertram. „Prädiktive Regelung mit Timed-Elastic-Bands“. In: at - Automatisierungstechnik Jg. 62, Nr. 10 (2014).

[Rös+15a] C. Rösmann, F. Hoffmann und T. Bertram. „Planning of multiple robot trajectories in distinctive topologies". In: 2015 European Conference on Mobile Robots (ECMR). 2015, S. 1-6.

[Rös+15b] C. Rösmann, F. Hoffmann und T. Bertram. „Timed-Elastic-Bands for timeoptimal point-to-point nonlinear model predictive control“. In: 2015 European Control Conference (ECC). 2015, S. 3352-3357.

[Sat+05] T. Sattel und T. Brandt. "Ground vehicle guidance along collision-free trajectories using elastic bands". In: Proceedings of the 2005 American Control Conference. 2005, S. 4991-4996.

[Sat+08] T. Sattel, T. Hesse und C. Sondermann-Wölke. „Automatisches Ausweichen in dynamischer Umgebung für Fahrerassistenzsysteme zur Kollisionsvermeidung". In: 3. Tagung Aktive Sicherheit durch Fahrerassistenz. 2008, o.S.

[Sch+06a] C. Schmidt, F. Oechsle und W. Branz. „Research on trajectory planning in emergency situations with multiple objects“. In: 2006 IEEE Intelligent Transportation Systems Conference. 2006, S. 988-992.

[Sch+06b] M. Schorn, U. Stahlin, A. Khanafer und R. Isermann. „Nonlinear trajectory following control for automatic steering of a collision avoiding vehicle“. In: 2006 American Control Conference. 2006, S. 5837-5842. 
[Sch+06c] M. Schorn und R. Isermann. "AUTOMATIC STEERING AND BRAKING FOR A COLLISION AVOIDING VEHICLE“. In: IFAC Proceedings Volumes Jg. 39, Nr. 16 (2006), S. 378-383.

[Sch+11] S. Schmidt, M. Schunemann und R. Kasper. „Path planning for a four wheel driven electric vehicle". In: 2011 IEEE/ASME International Conference on Advanced Intelligent Mechatronics (AIM). 2011, S. 790-795.

[Sch+12] S. Schmidt und R. Kasper. „Ein hierarchischer Ansatz zur optimalen Bahnplanung und Bahnregelung für ein autonomes Fahrzeug". In: at Automatisierungstechnik Jg. 60, Nr. 12 (2012), S. 743-754.

[Sch07] M. Schorn. „Quer- und Längsregelung eines Personenkraftwagens für ein Fahrerassistenzsystem zur Unfallvermeidung“. Dissertation. Technische Universität Darmstadt, 2007.

[Sch13] C. Schmidt. „Fahrstrategien zur Unfallvermeidung im Straßenverkehr für Einzel- und Mehrobjektszenarien“. Dissertation. Karlsruher Institut für Technologie, 2013.

[See+15] A. Seewald, C. Haß, M. Keller und T. Bertram. „Notausweichassistent zur Vermeidung von Kollisionen“. In: ATZ - Automobiltechnische Zeitschrift Jg. 117, Nr. 1 (2015), S. 26-30.

[Shi+10] T. Shim, G. Adireddy und H. Yuan. "Autonomous Vehicle Collision Avoidance Based Path Planning and MPC". In: 10th International Symposium on Advanced Vehicle Control. 2010, S. 731-736.

[Shi+12] T. Shim, G. Adireddy und H. Yuan. "Autonomous vehicle collision avoidance system using path planning and model-predictive-control-based active front steering and wheel torque control“. In: Proceedings of the Institution of Mechanical Engineers, Part D: Journal of Automobile Engineering Jg. 226, Nr. 6 (2012), S. 767-778.

[Sie+15] M. Sieber, K.-H. Siedersberger, A. Siegel und B. Farber. „Automatic Emergency Steering with Distracted Drivers: Effects of Intervention Design". In: 2015 IEEE 18th International Conference on Intelligent Transportation Systems (ITSC 2015). 2015, S. 2040-2045.

[Smi+95] D. E. Smith, J. M. Starkey und R. E. Benton. „Nonlinear-gain-optimized controller development and evaluation for automated emergency vehicle steering". In: 1995 American Control Conference - ACC'95. 1995, S. 35863591.

[Sou+10] D. Soudbakhsh, A. Eskandarian und D. Chichka. "Vehicle steering maneuvers with direct trajectory optimization“. In: 2010 IEEE Intelligent Vehicles Symposium (IV). 2010, S. 449-453.

[Sou+11] D. Soudbakhsh, A. Eskandarian und J. Moreau. „An emergency evasive maneuver algorithm for vehicles“. In: 2011 14th International IEEE Conference on Intelligent Transportation Systems - (ITSC 2011). 2011, S. 973-978. 
[Stä08] U. Stählin. „Eingriffsentscheidung für ein Fahrerassistenzsystem zur Unfallvermeidung“. Dissertation. Technische Universität Darmstadt, 2008.

[Tal+11] K. L. Talvala, K. Kritayakirana und J. C. Gerdes. „Pushing the limits: From lanekeeping to autonomous racing". In: Annual Reviews in Control Jg. 35, Nr. 1 (2011), S. 137-148.

[Tam+11] A. Tamke, T. Dang und G. Breuel. „A flexible method for criticality assessment in driver assistance systems". In: 2011 IEEE Intelligent Vehicles Symposium (IV). 2011, S. 697-702.

[Thi15] D. Thielsch. „Ein modellprädiktives Planungs- und Regelungsverfahren zur Fahrzeugführung in Notsituationen". Masterarbeit. Technische Universität Dortmund, 2015.

[Ulm94] B. Ulmer. „VITA II - active collision avoidance in real traffic“. In: Intelligent Vehicles '94 Symposium. 1994, S. 1-6.

[Wan+15] J. Wang, J. Wu und Y. Li. „The Driving Safety Field Based on DriverVehicle-Road Interactions". In: IEEE Transactions on Intelligent Transportation Systems Jg. 16, Nr. 4 (2015), S. 2203-2214.

[Web12] D. Weber. „Untersuchung des Potenzials einer Brems-Ausweich-Assistenz“. Dissertation. Karlsruher Institut für Technologie, 2012.

[Wei03] R. Weisen. „Gekoppelte Quer- und Längsregelung eines Personenkraftwagens im fahrphysikalischen Grenzbereich“. Dissertation. Universität der Bundeswehr Hamburg, 2003.

[Wer+10] M. Werling, J. Ziegler, S. Kammel und S. Thrun. „Optimal trajectory generation for dynamic street scenarios in a Frenét Frame“. In: 2010 IEEE International Conference on Robotics and Automation (ICRA 2010). 2010, S. 987993.

[Wer+12] M. Werling und D. Liccardo. "Automatic collision avoidance using modelpredictive online optimization“. In: 2012 IEEE 51st Annual Conference on Decision and Control (CDC). 2012, S. 6309-6314.

[Yi+16] B. Yi, S. Gottschling, J. Ferdinand, N. Simm, F. Bonarens und C. Stiller. „Real Time Integrated Vehicle Dynamics Control an Trajetory Planning with MPC for Critical Maneuvers". In: 2016 IEEE Intelligent Vehicles Symposium (IV). 2016, S. 584-589.

[Yoo+09] Y. Yoon, J. Shin, H. J. Kim, Y. Park und S. Sastry. „Model-predictive active steering and obstacle avoidance for autonomous ground vehicles". In: Control Engineering Practice Jg. 17, Nr. 7 (2009), S. 741-750.

[Zie+08] J. Ziegler und M. Werling. „Navigating car-like robots in unstructured environments using an obstacle sensitive cost function“. In: 2008 IEEE Intelligent Vehicles Symposium (IV). 2008, S. 787-791.

[Zie+09] J. Ziegler und C. Stiller. „Spatiotemporal state lattices for fast trajectory planning in dynamic on-road driving scenarios". In: 2009 IEEE/RSJ In- 
ternational Conference on Intelligent Robots and Systems (IROS 2009). 2009, S. $1879-1884$.

[Zie+14a] J. Ziegler, P. Bender, M. Schreiber, H. Lategahn, T. Strauss, C. Stiller, T. Dang, U. Franke, N. Appenrodt, C. G. Keller, E. Kaus, R. G. Herrtwich, C. Rabe, D. Pfeiffer, F. Lindner, F. Stein, F. Erbs, M. Enzweiler, C. Knoppel, J. Hipp, M. Haueis, M. Trepte, C. Brenk, A. Tamke, M. Ghanaat, M. Braun, A. Joos, H. Fritz, H. Mock, M. Hein und E. Zeeb. „Making Bertha Drive - An Autonomous Journey on a Historic Route“. In: IEEE Intelligent Transportation Systems Magazine Jg. 6, Nr. 2 (2014), S. 8-20.

[Zie+14b] J. Ziegler, P. Bender, T. Dang und C. Stiller. „Trajectory planning for Bertha - A local, continuous method“. In: 2014 IEEE Intelligent Vehicles Symposium (IV). 2014, S. 450-457. 


\section{Eigene wissenschaftliche Publikationen}

1. A. Homann, M. Keller, C. Götte, K.-H. Glander, C. Haß und T. Bertram „Kinodynamic Trajectory Optimization for Real-Time Vehicle Guidance - Kinodynamische Trajektorien Optimierung zur Echtzeit Fahrzeugführung“ In: VDI Automotive meets Eletronics 2017, 2017, S. 14 - 19.

2. C. Götte, M. Keller, C. Rösmann, T. Nattermann, C. Haß, K.-H. Glander, A. Seewald und T. Bertram. "A Real-Time Capable Model Predictive Approach to Lateral Vehicle Guidance“ In: 2016 IEEE Intelligent Transportation Systems Conference, 2016, S. 1908 - 1913.

3. C. Götte, M. Keller, C. Haß und T. Bertram. „Modellprädiktive Planung und Regelung im Anwendungsfall kritischer Verkehrssituationen“ In: ATZ - Automobiltechnische Zeitschrift, Jg. 118, Nr. 9, 2016, S. 68-73.

4. C. Götte, M. Keller, C. Haß, K.-H. Glander, A. Seewald und T. Bertram. „A Model Predictive Combined Planning and Control Approach for Guidance of Automated Vehicles" In: 2015 IEEE International Conference on Vehicular Electronics and Safety, 2015, S. 69-74.

5. M. Keller, C. Haß, A. Seewald und T. Bertram. „A Model Predictive Approach to Emergency Maneuvers in Critical Traffic Situations" In: 2015 IEEE 18th International Conference on Intelligent Transportation Systems - (ITSC 2015), 2015, S. 369-374.

6. M. Keller, C. Haß, A. Seewald und T. Bertram. „A vehicle lateral control approach for collision avoidance by emergency steering maneuvers" In: 6th International Munich Chassis Symposium 2015, 2015, S. 175-197.

7. M. Keller, C. Haß, A. Seewald und T. Bertram. „Ein modellprädiktives Planungsund Fahrzeugquerregelungsverfahren zur Kollisionsvermeidung durch Notausweichmanöver" In: VDI Automotive meets Electronics 2015, 2015, S. 8-13.

8. A. Seewald, C. Haß, M. Keller und T. Bertram. "Notausweichassistent zur Vermeidung von Kollisionen“ In: ATZ - Automobiltechnische Zeitschrift, Jg. 117, Nr. 1 (2015), S. 26-30.

9. M. Keller, C. Haß, A. Seewald und T. Bertram. „Driving Simulator Study on an Emergency Steering Assist" In: 2014 IEEE International Conference on Systems, Man and Cybernetics - SMC, 2014, S. 3008-3013.

10. M. Keller, F. Hoffmann, T. Bertram, C. Hass und A. Seewald. „Planning of Optimal Collision Avoidance Trajectories with Timed Elastic Bands" In: 19th IFAC World Congress 2014, 2014, S. 9822-9827.

11. M. Keller, C. Haß, A. Seewald und T. Bertram. „Sensitivitätsanalyse der die Fahrzeugdynamik beeinflussenden Systemparameter" In: VDI Automotive meets Electronics 2014, 2014, S. 141-146. 
12. M. Keller, C. Haß, A. Seewald und T. Bertram. „Ein Regelungskonzept für eine Notausweichassistenz" In: VDI AUTOREG 2013, Bd. VDI Berichte Nr. 2196, 2013, S. 363-372.

\section{Weitere Vorträge und Posterbeiträge}

1. M. Keller, C. Haß und T. Bertram. „Assistiertes Notausweichen - Die Alternative zur Notbremsung“. 2. BusinessForum21-Jahrestagung, 2016

2. M. Keller, A. Homann, C. Götte, C. Haß und T. Bertram. „Fahrdynamikregelung zur Kollisionsvermeidung mit Timed Elastic Bands". 11. Dortmunder AutoTag, 2016

3. M. Keller, C. Haß und T. Bertram. "Automatische Notfall-Lenkhilfe für PKW“. Hochschul Wettbewerb ZukunftErfindenNRW, 2015

4. M. Keller, C. Haß, A. Seewald und T. Bertram. „Verschiedene Verfahren für ein Fahrerassistenzsystem zur Kollisionsvermeidung". 10. Dortmunder AutoTag, 2015

5. M. Keller, C. Haß, A. Seewald und T. Bertram. „Emergency Steering Assist ESA Fahrzeugquerregelung durch Lenkmomentenüberlagerung". VDE Vortragskarussell, 2015

6. M. Keller, C. Haß, A. Seewald und T. Bertram. „Fahrsimulator-Studien mit einem Notausweichassistenten“. Automotive Innovationsforum. 2014

7. M. Keller, F. Hoffmann, T. Bertram, C. Haß und A. Seewald. „Planung optimaler Bremsausweichmanöver mit Timed Elastic Bands“. 9. Dortmunder AutoTag, 2014

8. M. Keller, C. Haß, A. Seewald und T. Bertram. „Sensitivitätsanalyse der Querdynamik von Personenkraftwagen“. 8. Dortmunder AutoTag, 2013

9. M. Keller, C. Haß und T. Bertram. „Vergleich von Regelungsgrößen für eine Notlenkassistenz". 7. Dortmunder AutoTag, 2012

\section{Patentanmeldungen}

1. C. Haß, T. Bertram und M. Keller. „Steuergerät und Verfahren für eine NotfallLenkunterstützungsfunktion“ Pat. WO 2014/195087 A1, DE 102013009252 A1, US2016/280265 A1, CN 105813914 A, 2014. 


\section{Betreute Abschlussarbeiten}

1. Alexander Hugenroth. „Simulationsbasierte Untersuchung der modellprädiktiven Trajektorienscharregelung für ein hydraulisches Wegeventil“. Bachelorarbeit. 2016

2. Hossein Taherian. „Lokale und globale Sensitivitätsanalyse der physikalischen Parameter eines elektromechanischen Lenksystems“. Bachelorarbeit. 2016

3. Daniel Spitzlei. „Entwicklung, Analyse und Vergleich von Fahrdynamikregelungen zur Trajektorienfolge“. Masterarbeit. 2016

4. Dennis Thielsch. „Ein modellprädiktives Planungs- und Regelungsverfahren zur Fahrzeugführung“. Masterarbeit. 2015

5. Andreas Homann. „Ein Verfahren zur Fahrzeugführung mittels echtzeitfähiger Trajektiorienoptimierung und Fahrdynamikregelung“. Masterarbeit. 2015

6. Henning Hartmann. „Entwicklung und Untersuchung eines modellprädiktiven Trajektorienscharplanungs- und Fahrzeugquerregelungsverfahrens zur Unfallvermeidung im Straßenverkehr durch Notausweichmanöver". Bachelorarbeit. 2014

7. Christian Götte. „Integration einen Fahrdynamikmodells in das Timed Elastic Band Verfahren zur Durchführung optimaler Bremsausweichmanöver“. Masterarbeit. 2014

8. Waldemar Schulz. „Modellbildung von Bremsanalgen in batterieelektrischen Fahrzeugen und Entwicklung einer Rekuperationsstrategie“. Bachelorarbeit. 2014

9. Markus Buß. „Modellprädiktive Regelung der Querdynamik von Kraftfahrzeugen entlang vorgegebener Bahnen im fahrphysikalischen Grenzbereich“. Masterarbeit. 2013

10. Andreas Homann. „Beschreibung von optimalen Bremsausweichtrajektorien in kritischen Verkehrssituationen“. Bachelorarbeit. 2013

11. Dennis Thielsch. „Entwicklung eines Fahrermodells zur Fahrzeuglängsführung von Elektrofahrzeugen“. Bachelorarbeit. 2012

\section{Betreute Projektgruppen}

1. Moritz Lutkemöller, Dennis Thielsch, Patrick Klokowski, Tobias Kuhl, Indra Adi Marta, Juan Xu. „Einsatz eines Fahrsimulators zur Entwicklung fortschrittlicher Fahrerassistenzsysteme“. 2014 